\title{
A Trip to Remember
}

Citation for published version (APA):

McCrum, C. (2019). A Trip to Remember: Assessing and Improving Walking Stability in Older Adults.

[Doctoral Thesis, Maastricht University]. Gildeprint Drukkerijen. https://doi.org/10.26481/dis.20191219cm

Document status and date:

Published: 01/01/2019

DOI:

$10.26481 /$ dis. $20191219 \mathrm{~cm}$

Document Version:

Publisher's PDF, also known as Version of record

\section{Please check the document version of this publication:}

- A submitted manuscript is the version of the article upon submission and before peer-review. There can be important differences between the submitted version and the official published version of record.

People interested in the research are advised to contact the author for the final version of the publication, or visit the DOI to the publisher's website.

- The final author version and the galley proof are versions of the publication after peer review.

- The final published version features the final layout of the paper including the volume, issue and page numbers.

Link to publication

\footnotetext{
General rights rights.

- You may freely distribute the URL identifying the publication in the public portal. please follow below link for the End User Agreement:

www.umlib.nl/taverne-license

Take down policy

If you believe that this document breaches copyright please contact us at:

repository@maastrichtuniversity.nl

providing details and we will investigate your claim.
}

Copyright and moral rights for the publications made accessible in the public portal are retained by the authors and/or other copyright owners and it is a condition of accessing publications that users recognise and abide by the legal requirements associated with these

- Users may download and print one copy of any publication from the public portal for the purpose of private study or research.

- You may not further distribute the material or use it for any profit-making activity or commercial gain

If the publication is distributed under the terms of Article $25 \mathrm{fa}$ of the Dutch Copyright Act, indicated by the "Taverne" license above, 


\section{A Trip to Remember: \\ Assessing and Improving \\ Walking Stability in Older Adults}

Christopher McCrum 
The studies presented in this dissertation were performed within NUTRIM School of Nutrition and Translational Research in Metabolism.

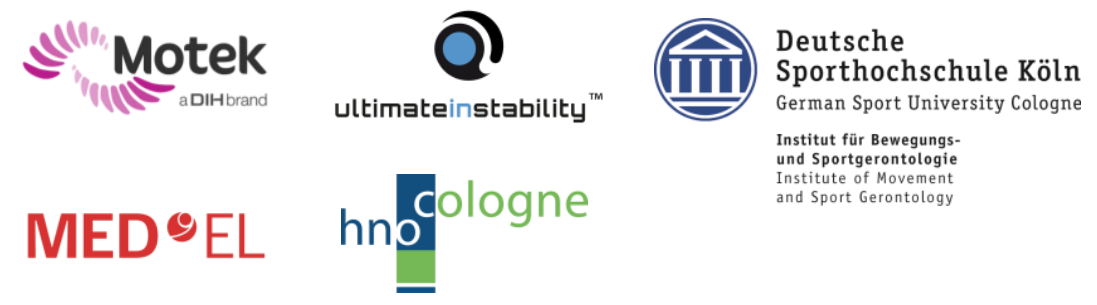

Financial support for the printing of this dissertation from Maastricht University and the following sponsors is gratefully acknowledged: Motek, Ultimateinstability, das Institut für Bewegungs- und Sportgerontologie (Deutsche Sporthochschule Köln), MED-EL and Prof. Dr. K. Eysel-Gosepath (HNO Cologne).

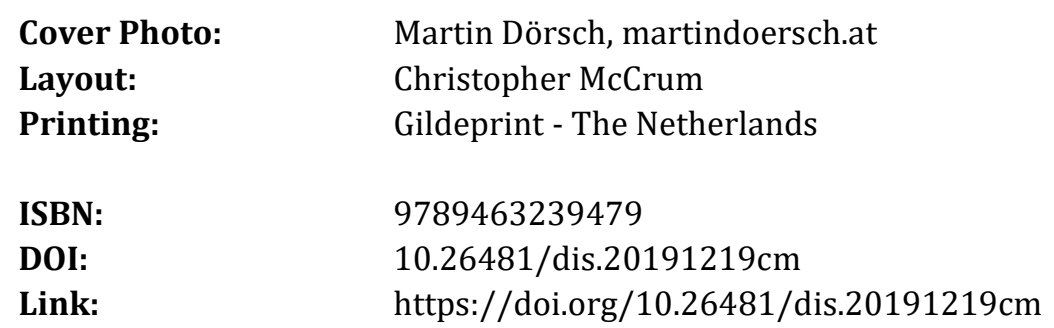

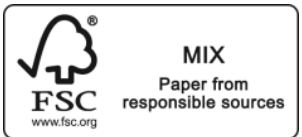

(C) Christopher McCrum 2019.

Parts One, Six and Seven of this work are licensed under the Creative Commons Attribution 4.0 International License (http://creativecommons.org/licenses/by/4.0/) which permits unrestricted use, distribution, and reproduction in any medium, provided the original work is properly cited.

The licences of the chapters in Parts Two, Three, Four and Five of this dissertation are indicated on the individual chapter pages. 


\section{A Trip to Remember: Assessing and Improving Walking Stability in Older Adults}

\section{DISSERTATION}

to obtain the degree of Doctor at the Maastricht University, on the authority of the Rector Magnificus, Prof. dr. Rianne M. Letschert in accordance with the decision of the Board of Deans, to be defended in public on Thursday, 19 $9^{\text {th }}$ of December 2019 at 14:00 hours.

By

\section{Christopher McCrum}

Born on the $4^{\text {th }}$ of August 1990 in Glasgow, Scotland. 


\section{Supervisors}

Dr. K. Meijer

Prof. Dr. K. Karamanidis (London South Bank University, London, United Kingdom)

\section{Co-Supervisor}

Dr. R. van de Berg

\section{Assessment Committee}

Prof. Dr. R. J. Vermeulen (chair)

Univ. Prof. Dr. U. G. Kersting (German Sport University Cologne, Cologne, Germany)

Prof. Dr. A. F. Lenssen

Prof. Dr. M. Pijnappels (VU Amsterdam, Amsterdam, The Netherlands)

Prof. Dr. H. H. C. M. Savelberg 


\section{Table of Contents}

Part One: General Introduction $\quad 1$

1.1: World Population Ageing 2

1.2: Consequences of Falls among Older Adults 2

1.3: Incidence of Falls among Older Adults 3

1.4: Common Causes of Falls among Older Adults 5

1.5: Sensing Balance Loss in Old Age $\quad 7$

1.6: Gait Stability and Adaptability 8

1.7: Aims and Outline of the Dissertation 13

Part Two: Unperturbed and Predictive Gait Stability with Ageing and Vestibulopathy 15

2.1: The walking speed-dependency of gait variability in bilateral vestibulopathy and its association with clinical tests of vestibular function 17

2.2: Locomotor stability and adaptation during perturbed walking across the adult female lifespan 39

Part Three: Gait Perturbation Methodology $\mathbf{5 1}$

3.1: A systematic review of gait perturbation paradigms for improving reactive stepping responses and falls risk among healthy older adults $\quad 53$

3.2: Stability-normalised walking speed: a new approach for human gait perturbation research 71

Part Four: Reactive Gait Stability and Adaptability

4.1: Retention, savings and interlimb transfer of reactive gait adaptations in humans following unexpected perturbations

4.2: Older adults demonstrate interlimb transfer of reactive gait adaptations to repeated unpredictable gait perturbations $\quad 113$

Part Five: Improving the Reactive Stability of Older Adults

5.1: Perturbation-based balance training for falls reduction among older adults: current evidence and implications for clinical practice

Part Six: General Discussion

6.1: Summary of the Main Findings $\quad 166$

6.2: Exercise-based Fall Risk Assessment and Intervention $\quad 166$

6.3: Challenges in Implementing Fall Risk Assessment and Reduction Interventions 173

6.4: Future Directions in Specific Populations 174

6.5: Conclusions of the General Discussion 176

$\begin{array}{lr}\text { Part Seven: Summaries and Valorisation } & 179\end{array}$

7.1: English Summary $r$

7.2: Nederlandse Samenvatting $\quad 182$

7.3: Deutsche Zusammungfassung $\quad 184$

$\begin{array}{lr}\text { 7.4: Valorisation } & 188\end{array}$

References $\quad 193$

$\begin{array}{ll}\text { Acknowledgements } & 233\end{array}$

$\begin{array}{lr}\text { Curriculum Vitae } & 241\end{array}$

Publication List $\quad 242$

About the Author $\quad 249$ 



\section{Part One:}

\section{General Introduction}

"It's a dangerous business, Frodo, going out your door," he used to say. "You step into the Road, and if you don't keep your feet, there is no knowing where you might be swept off to."

- Frodo Baggins quoting Bilbo Baggins. The Fellowship of the Ring. 


\section{1: World Population Ageing}

People are living longer than ever before, and this trend is only going to continue in the future (Kinsella et al., 2000, Lutz et al., 2008). In 2017, the global population over the age of 60 years was more than twice what it was in 1980, and by 2050, is due to double again (United Nations, 2017). This increase in the number of older adults in the coming years will occur in both developed and developing countries. In the USA, the population aged over 65 years is predicted to be 83.7 million in 2050, almost double what it was in 2012 (43.1 million; Ortman et al., 2014). In the UK, more than $70 \%$ of the increase in the population between 2014 and 2039 is predicted to be in the over 60 years age group (Harper and Walport, 2016). Across the European Union, similar projections have also been predicted (Guerin et al., 2015). The numbers of older adults in developing regions of the world are also growing and are predicted to continue to grow faster than in developed countries (United Nations, 2017). By 2050, almost 50\% of the world's population will live in countries with at least $20 \%$ of their populations aged 60 years or older, and $25 \%$ of people will live in a country where people of 60 years make up more than $30 \%$ of the population. As a result, global ageing presents significant challenges for health, long term care and welfare systems (Kinsella et al., 2000, Lutz et al., 2008, Rechel et al., 2013, Harper and Walport, 2016, United Nations, 2017).

\section{2: Consequences of Falls among Older Adults}

As early as the 1950's and 1960's, falls were already examined and discussed in the scientific and medical literature as a common health risk to older adults (Howell, 1958, Fine, 1959, Sheldon, 1960). A fall is commonly defined as "...an unexpected event in which the participants come to rest on the ground, floor, or lower level." (Lamb et al., 2005). Ageing is significantly associated with increasing fall risk (Talbot et al., 2005, Deandrea et al., 2010, Schumacher et al., 2014, Kwan et al., 2016). Due to global population ageing, there is increasing concern about falls and their consequences, in both developed and developing countries (Kalula et al., 2011, Stewart Williams et al., 2015).

Falls represent one of the most common causes of injury among older adults (Ghodsi et al., 2003, Xu and Drew, 2016) and can significantly impair mobility and quality of life for long periods of time following the fall (Grisso et al., 1992, Kosorok et al., 1992, Chu et al., 2006, Hartholt et al., 2011). The risk of injury requiring hospital admission following a fall also significantly rises with increasing age within older adults (Sattin et al., 1990, Malmivaara et al., 1993), with one study reporting $44 \%$ of accident and emergency admissions in older adults being the result of a fall (Davies and Kenny, 1996). There is also a risk of mortality due to injuries sustained from a fall (Sattin et al., 1990, Malmivaara et al., 1993, Terroso et al., 2013). For example, in Scotland from 2010-2011, 46,816 people visited hospital due to a fall and 16,549 of those people were admitted (Craig et al., 2013). Of these 16,549 people admitted to hospital, $7 \%$ died during the hospital stay and an additional 5\% died over the following 12 months (Craig et al., 2013).

Concerningly, the number of fall-related injuries has been rising in recent years. For example, fall-related injuries increased from 2005-2013 in Canada (Do et al., 2015), and from 2008-2013 in South Korea (Hong et al., 2016). Hospital admission rates as a 
result of fall-related injuries rose in the Netherlands from 1981-2008 and in the USA from 2001-2012 (Hartholt et al., 2010, Orces and Alamgir, 2014). In Finland, more cervical spine, spinal cord and head injuries due to falls occurred over time (Kannus et al., 2000, Kannus et al., 2007a, Kannus et al., 2007b). As well as the mounting number of injuries due to falls, fall-related deaths increased by 31\% between 2007 and 2016 in the USA (Burns and Kakara, 2018). The most recent analysis in the Netherlands found that from 1997 to 2016, absolute numbers of emergency department visits, hospital admissions and deaths related to falls grew by $48 \%, 59 \%$, and $267 \%$, respectively (Olij et al., 2019).

These injuries and hospital admissions also come with a huge financial burden (Stevens et al., 2006, Burns et al., 2016). In the Netherlands, between 2003-2007, falls led to a total estimated healthcare cost of $€ 474.4$ million (Hartholt et al., 2011), which was exceeded by a later estimate of $€ 675.4$ million annually from 2007-2009 (Hartholt et al., 2012). In the USA, non-fatal and fatal falls cost $\$ 23.3$ billion annually and $\$ 1.6$ billion in the UK (2008 prices; Davis et al., 2010). The estimated cost per fall has been estimated at $€ 9,370$ in the Netherlands (Hartholt et al., 2012) and between $\$ 4,291$ and $\$ 4,642$ in Australia (Hall and Hendrie, 2003). In Scotland and the USA, respectively, cost per fall has been estimated to be over $£ 1,720$ and $\$ 3,476$, and to over $£ 8,600$ and $\$ 10,749$ per injurious fall needing medical assistance (Davis et al., 2010, Craig et al., 2013). For falls requiring hospital admission, the estimated costs were $£ 39,490$ (hip fractures) or $£ 21,960$ (other injuries), and $\$ 26,483$ in Scotland and the USA, respectively (Davis et al., 2010, Craig et al., 2013).

In summary, falls represent a major burden to our ageing populations. This burden includes injuries and reductions in quality of life to the individuals experiencing the fall, as well as societal and financial costs related to the care for individuals who have experienced falls, and these seem to be increasing over time with our ever-expanding ageing populations.

\section{3: Incidence of Falls among Older Adults}

Falls are a persistent problem in older populations and have been for some time. In order to provide an up to date overview, a semi-structured literature search for articles investigating falls incidence over one year in older adults was conducted. Studies that included at least 100 participants and both males and females were included, to ensure relatively reliable and representative data would be included in this overview. Results from a Web of Science search with

TITLE: (falls) AND TOPIC: ((older adults) OR (elderly))

was used to identify the top 50 cited articles matching these criteria and the following PubMed search string

(prospective[Title/Abstract] AND (falls[Title] OR fall[Title])) AND (older adults[Title/Abstract] OR elderly[Title/Abstract] OR aged[Title/Abstract])

were combined with articles known to the author. Snowball searches using reference lists and forward citation searches in PubMed and Web of Science were conducted for each article identified from the two searches. Table 1 contains the studies found that 
determined falls incidence retrospectively and Table 2 contains those which prospectively monitored falls incidence. Fig. 1 provides an overview of the falls incidence over time reported in the included studies.

In the included studies, a broad range of fall incidences was found, from $13 \%$ to $60.3 \%$ in the retrospective studies and from $19.3 \%$ to $62.5 \%$ in the prospective studies (Tables 1 and 2; Fig. 1). As has been previously noted by Lord et al. (2011), participants originating in east Asian countries appear to have lower falls incidence than European, mainland North American and Australasian participants, with approximate average percentages of participants experiencing one or more falls in a 12-month period of $19 \%$ for retrospective studies in east Asian populations and 29\% for European, mainland North American and Australasian populations. Retrospective studies in South American populations also show an approximate average percentage of $30 \%$ and two studies in Caribbean populations show approximately $23 \%$ of participants experiencing one or more falls in a 12-month period. Of the prospective studies, an approximate average percentage of $40 \%$ of participants experiencing one or more falls was found in European, mainland North American and Australasian populations. Additionally, one prospective study in China reported 19.3\%, one in Japan reported 34.5\% and one study in Brazil reported 47.4\% of older adults experiencing a fall over 12 months.

Despite the variation across populations in falls incidence and the potential differences in falls assessment methodology (Cummings et al., 1988, Rapp et al., 2014, Griffin et al., 2019), the incidence rates found are high enough to be concerning and there

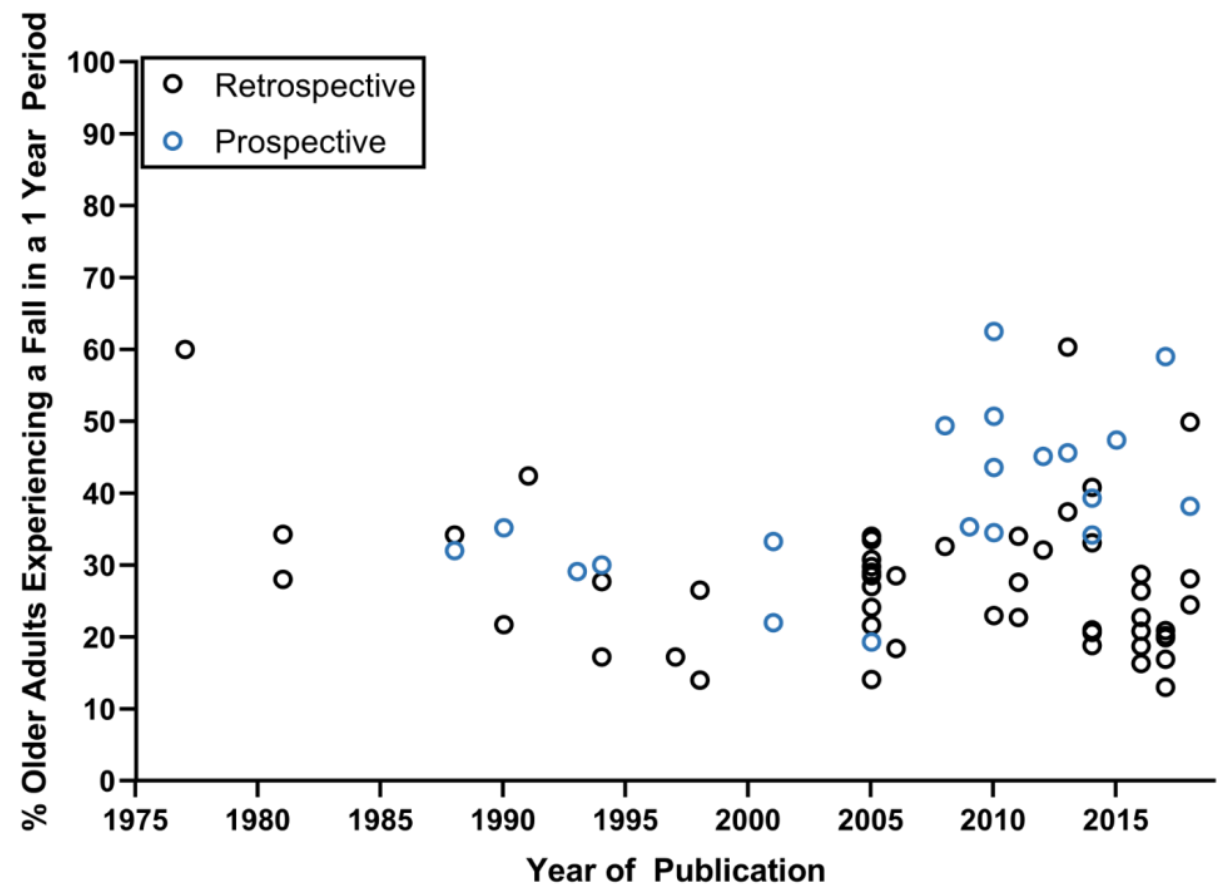

Fig. 1: Summary of the results of the literature search for the percentage of older adults who experienced a fall in the previous 12 months (retrospective) or who experienced a fall over 12 months during the study (prospective), plotted against the year of publication. Note that the periods of data collection, when reported, sometimes differ slightly and these data can be found in Tables 1 and 2 . 
is not an obvious trend to suggest any major changes in falls incidence over the last 45 years. Combined with the increasing incidence in fall-related injuries discussed above, this suggests that despite the knowledge gained over this period and the proliferation and awareness of research into falls, interventions to reduce falls among older adults are not effective enough to have a meaningful influence on daily life falls or are not accessible to a large enough percentage of older adults to have a meaningful impact on population level falls statistics, or both. The research described in this dissertation relates to the first possibility; that interventions conducted in the past may not have been effective enough to have the most positive effect on falls incidence possible. The first step to evaluate this possibility is to look at the situations that lead to falls in older adults, in attempt to determine what factors might serve as appropriate targets for intervention.

\section{4: Common Causes of Falls among Older Adults}

It has been consistently reported that, among older adults as well as middle-aged and younger adults, walking is the most common activity preceding falls (Berg et al., 1997, Hill et al., 1999, Niino et al., 2000, Talbot et al., 2005, Li et al., 2006, Muir et al., 2008, Kelsey et al., 2012, Robinovitch et al., 2013, van Schooten et al., 2018, Yang et al., 2018b), and the association between walking and falls has been recognised for some time (Howell, 1958, Sheldon, 1960, Azar and Lawton, 1964). Consequently, it is unsurprising that walking often precedes falls that have led to injuries requiring admittance to accident and emergency departments (Fothergill et al., 1995). Timsina et al. (2017) reported that of 4,135 fall-related injury reports between 1997-2010, walking was the most common activity at the time of the fall for all ages and genders, except young men, who were more commonly engaged in vigorous activity at the time of the fall. Falls during walking are also related to the risk of sustaining a hip fracture (Aharonoff et al., 1998, Abolhassani et al., 2006, Boye et al., 2014). However, while walking commonly precedes falls, it is not the act of walking itself that usually causes falls among older adults.

"Any observer who has watched an older person trying to board a bus which is just starting to move, will have noted the slow reflexes and the liability to fall which occur as the result of these changes." Howell (1958)

As this observation from Howell indicates, people have appreciated for some time that external, sudden changes in the immediate environment while ambulating pose a threat to older adults. While this was simply Howell's observation, one of, if not, the earliest systematic accounts of causes of falls among older adults supported the observation very soon afterwards. Sheldon (1960), who reviewed the causes of 500 falls in 202 older individuals (50 years and older), reported stair walking, slipping, tripping and falling over unexpected objects to be among the most common causes of falls, alongside poor lighting and vertigo. Since then, trips and slips have often been reported to be the most common causes of falls among older adults (Tinetti et al., 1988, Lord et al., 1993, Berg et al., 1997, Niino et al., 2000, Talbot et al., 2005). Trips and slips have also been reported to be the most common causes of falls leading to injuries (Roudsari et al., 2005, Gilasi et al., 2015). 
Table 1. Retrospective studies of falls incidence over 12 months in older adults

\begin{tabular}{|c|c|c|c|c|c|}
\hline Study & Data Collection & $\mathbf{N}$ & Age & \%Fallers & Country \\
\hline $\begin{array}{l}\text { Prudham and Evans } \\
\text { (1981) }\end{array}$ & $1975-1977$ & 2,357 & $\geq 65$ years & $28 \%$ & UK \\
\hline Overstall et al. (1977) & $\begin{array}{l}\text { Not stated. Accepted } \\
1976\end{array}$ & 243 & $60-96$ & $60 \%$ & UK \\
\hline Campbell et al. (1981) & $\begin{array}{l}\text { Not stated. } \\
\text { Published } 1981\end{array}$ & 553 & $\geq 65$ years & $34.3 \%$ & New Zealand \\
\hline Blake et al. (1988) & 1985 & 1,042 & $\geq 65$ years & $34.2 \%$ & UK \\
\hline $\begin{array}{l}\text { Downton and Andrews } \\
\text { (1991) }\end{array}$ & $1986-1988$ & 203 & $\geq 75$ years & $42.4 \%$ & UK \\
\hline Teno et al. (1990) & 1988 & 586 & $\geq 65$ years & $21.7 \%$ & USA \\
\hline Yasumura et al. (1994) & 1991 & 807 & $65-84$ years & $17.2 \%$ & Japan \\
\hline Chan et al. (1997) & 1992-1993 & 401 & $\geq 60$ years & $17.2 \%$ & Singapore \\
\hline Aoyagi et al. (1998) & $1992-1994$ & 1,054 & $\geq 65$ years & $14 \%$ & $\begin{array}{l}\text { USA (Japanese-Americans } \\
\text { living in Hawaii) }\end{array}$ \\
\hline $\begin{array}{l}\text { Baumgartner et al. } \\
\text { (1998) }\end{array}$ & 1993-1995 & 808 & $\begin{array}{l}\text { Mean } 73.6 \\
\text { years }\end{array}$ & $26.5 \%$ & USA \\
\hline Lord et al. (1994a) & Not stated. & 1,762 & $\geq 60$ years & $27.7 \%$ & Australia \\
\hline Aoyagi et al. (1998) & 1994 & 1,534 & $\geq 65$ years & $14 \%$ & Japan \\
\hline Chu et al. (2005) & 1998-1999 & 1,517 & $\geq 65$ years & $14.1 \%$ & China \\
\hline Sayer et al. (2006) & $1998-2004$ & 2,148 & 59-73 years & $18.4 \%$ & UK \\
\hline Chien and Guo (2014) & 1999 & 3,497 & $\geq 65$ years & $18.8 \%$ & Taiwan \\
\hline \multirow{8}{*}{$\begin{array}{l}\text { Reyes-Ortiz et al. } \\
(2005)\end{array}$} & $1999-2000$ & 964 & $\geq 60$ years & $28.5 \%$ & Argentina \\
\hline & $1999-2000$ & 1,635 & $\geq 60$ years & $21.6 \%$ & Barbados \\
\hline & $1999-2000$ & 1,777 & $\geq 60$ years & $29 \%$ & Brazil \\
\hline & $1999-2000$ & 1,205 & $\geq 60$ years & $34 \%$ & Chile \\
\hline & $1999-2000$ & 1,727 & $\geq 60$ years & $24.1 \%$ & Cuba \\
\hline & $1999-2000$ & 1,062 & $\geq 60$ years & $33.5 \%$ & Mexico \\
\hline & $1999-2000$ & 1,395 & $\geq 60$ years & $27 \%$ & Uruguay \\
\hline & $2000-2001$ & 1,483 & $\geq 71$ years & $30.8 \%$ & USA (Mexican-Americans) \\
\hline Gill et al. (2005) & 2000 & 2,619 & $\geq 65$ years & $29.8 \%$ & Australia \\
\hline Mancini et al. (2005) & 2002 & 2,273 & $\geq 65$ years & $28.6 \%$ & Italy \\
\hline Palumbo et al. (2016) & $2002-2003$ & 892 & $\geq 68$ years & $20.8 \%$ & Italy \\
\hline Halil et al. (2006) & $2002-2004$ & 2,322 & $\geq 65$ years & $28.5 \%$ & Turkey \\
\hline $\begin{array}{l}\text { Bekibele and Gureje } \\
(2010)\end{array}$ & $2003-2004$ & 2,096 & $\geq 65$ years & $23 \%$ & Nigeria \\
\hline Palumbo et al. (2016) & 2006 & 3,303 & $\geq 65$ years & $22.7 \%$ & UK \\
\hline Kojima et al. (2008) & $2006-2007$ & 849 & $\geq 65$ years & $32.6 \%$ & Japan \\
\hline $\begin{array}{l}\text { Almawlawi et al. } \\
\text { (2011) }\end{array}$ & 2008 & 355 & $\geq 60$ years & $34 \%$ & Qatar \\
\hline Verma et al. (2016) & 2008 & 5,808 & $\geq 65$ years & $16.3 \%$ & USA \\
\hline Lin et al. (2011) & 2008 & 1,377 & $\geq 65$ years & $22.7 \%$ & Taiwan \\
\hline Du et al. (2017) & $2008-2011$ & 1,833 & $65-79$ & $20.2 \%$ & Germany \\
\hline Rapp et al. (2014) & $2008-2011$ & 1,998 & $65-80$ years & $21 \%$ & Germany \\
\hline Orces (2013) & 2009 & 5,227 & $\geq 60$ years & $37.4 \%$ & Ecuador \\
\hline Bhangu et al. (2017) & 2009-2011 & 3,511 & $\geq 65$ years & $19.9 \%$ & Ireland \\
\hline Rapp et al. (2014) & $2009-2010$ & 1,388 & $65-90$ years & $33.1 \%$ & Germany \\
\hline Kalula et al. (2016) & $2009-2010$ & 837 & $\geq 65$ years & $26.4 \%$ & South Africa \\
\hline Cruz et al. (2012) & 2010 & 420 & $\geq 60$ years & $32.1 \%$ & Brazil \\
\hline Kamel et al. (2013) & $2010-2011$ & 340 & $\geq 60$ years & $60.3 \%$ & Egypt \\
\hline Choi et al. (2014) & 2011 & 43,367 & $\geq 65$ years & $20.6 \%$ & South Korea \\
\hline Siqueira et al. (2011) & Not stated. & 6,616 & $\geq 60$ years & $27.6 \%$ & Brazil \\
\hline Sharma et al. (2017) & 2012 & 561 & $\geq 60$ years & $13 \%$ & India \\
\hline Kang et al. (2017) & 2013-2014 & 541 & $\geq 60$ years & $20.9 \%$ & China \\
\hline Vieira et al. (2018) & 2014 & 1,448 & $\geq 60$ years & $28.1 \%$ & Brazil \\
\hline Bergen et al. (2016) & 2014 & 147,316 & $\geq 65$ years & $28.7 \%$ & USA \\
\hline Allain et al. (2014) & Not stated. & $98^{*}$ & $\geq 60$ years & $40.8 \%$ & Malawi \\
\hline Kim et al. (2017) & $2014-2015$ & 307 & $\geq 65$ years & $16.9 \%$ & South Korea \\
\hline Wang et al. (2016) & $\begin{array}{l}\text { Not stated. } \\
\text { Submitted } 2015\end{array}$ & 1,092 & $\geq 60$ years & $18.7 \%$ & China \\
\hline Almegbel et al. (2018) & 2016 & 1,182 & $\geq 60$ years & $49.9 \%$ & Saudi Arabia \\
\hline Alex et al. (2018) & $\begin{array}{l}\text { Not stated. } \\
\text { Submitted } 2017\end{array}$ & 1,051 & $\geq 65$ years & $24.5 \%$ & Malaysia \\
\hline
\end{tabular}

\footnotetext{
*: Lower than the inclusion criteria but included on the basis of the limited number of studies in African countries.
} 
Table 2. Prospective studies of falls incidence over 12 months in older adults

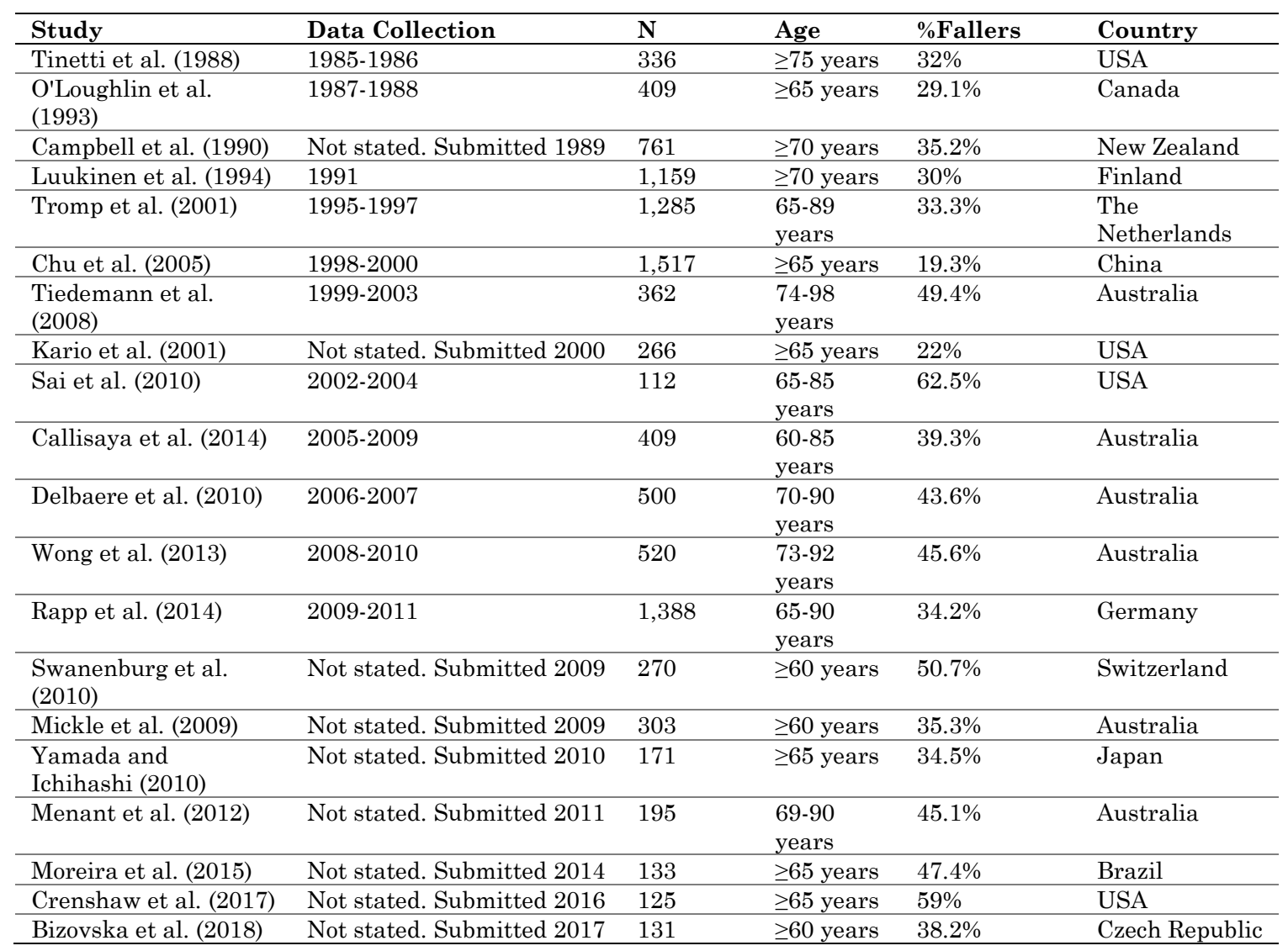

For example, in 2010 in Poland, approximately 70\% (31,712) of hospital admissions due to falls among older adults were due to a trip or slip-related fall (Buczak-Stec and Gorynski, 2013). It has also been reported that slips and trips are common causes of fall-related injuries in all age groups and genders, with more trip-related injuries occurring in females than in males (Timsina et al., 2017). It is also worth noting that differences in the circumstances of falls can be found when looking at different populations. For example, in long term care settings, walking seems to remain the most common activity prior to falls, as in community dwelling older adults, but falls often occur due to incorrect bodyweight shifting, rather than as a result of tripping or slipping (Robinovitch et al., 2013, Yang et al., 2018b). However, individual differences may also play a role, as more frequent fallers appear to fall more during sit-to-stand transfers than during walking, while less frequent fallers do appear to fall most often during walking (van Schooten et al., 2018). Taken together, the above results generally indicate that mechanical disturbances during walking pose a significant threat to older adults in terms of risk of falling.

\section{5: Sensing Balance Loss in Old Age}

When considering the ability to cope with sudden, unexpected mechanical disturbances to walking, the overall success of the response can be affected by how quickly and accurately a threat is perceived and therefore, the sensory systems play a critical role in balance 
recovery performance (Nutt et al., 1993, Sousa et al., 2012, MacKinnon, 2018). This section briefly outlines the systems involved and how they are related to ageing and falls.

If we take the example of a person experiencing forward balance loss due to a trip, the proprioceptive system will detect the contact with the object tripping the swing limb, as well as departures in limb and joint positions from their expected configurations during normal gait. The vestibular system will detect changes in the orientation and acceleration of the head in space with respect to gravity as a result in the increased forward acceleration and rotation. Finally, the visual system will perceive the change in visual surroundings as a result of the sudden diversion in trajectory. These sensory inputs will trigger specific reflexes for supporting balance and additionally will be integrated and weighted by the central nervous system in order to produce appropriate motor responses (Nutt et al., 1993, Nielsen and Sinkjaer, 2002, Sousa et al., 2012, MacKinnon, 2018).

It is well established that with age, a decline in sensory function occurs (For overviews, see: Schultz, 1992, Maki and McIlroy, 1996, Whitney and Morris, 2006, Lord et al., 2011, Agrawal et al., 2018, Lord et al., 2018, Paraskevoudi et al., 2018) and many of these declines in function have been linked to fall risk. For example, poor vision has been associated with an increased risk of falls (Lord et al., 1994a, Jack et al., 1995, Lord et al., 2011), in particular contrast sensitivity and depth perception (Lord et al., 1994b, Maki and McIlroy, 1996, King, 2009). Regarding the proprioceptive system, sensitivity to foot position is reduced in old age (Robbins et al., 1995, Ashton-Miller and Alexander, 2009) which may affect the ability to perceive mechanical disturbances to the lower limbs. Decreased muscle spindle activity and sensitivity of skin and joint receptors (Lord et al., 1994b, Whitney and Morris, 2006) as well as the presence of peripheral neuropathy and reduced foot touch sensation (Lord et al., 1994a, Richardson and Hurvitz, 1995, Lipsitz et al., 2018) have been linked to an increased risk of falls in older adults. Finally, there is a reduction in vestibular function with age (recently termed and classified as "presbyvestibulopathy"; (Agrawal et al., 2018)) and an increased prevalence of vestibular disorders with increasing age (For reviews, see: Anson and Jeka, 2015, Iwasaki and Yamasoba, 2015, Zalewski, 2015, Arshad and Seemungal, 2016, Ji and Zhai, 2018). Asymmetry or reduction in vestibular function has been related to falls history and future incidence among older adults (Menant et al., 2012, Ekvall Hansson and Magnusson, 2013, Liston et al., 2014, Larsson et al., 2015). Additionally, a previous study of our group found that when middle-aged participants with unilateral vestibulopathy were exposed to a cable-trip perturbation during treadmill walking, they were significantly less stable and required more steps to recover from the first trip compared to age-matched controls (McCrum et al., 2014). In Chapter 2.1 of this dissertation, the role of the vestibular system in gait is explored further.

\section{6: Gait Stability and Adaptability}

The topic of gait stability crosses several fields, both fundamental and applied, including neuroscience, motor control and biomechanics, as well as physical therapy, rehabilitation and geriatrics. It is therefore unsurprising that the term "gait stability" can mean different things, in different contexts, to different people (the same can be said for the term 


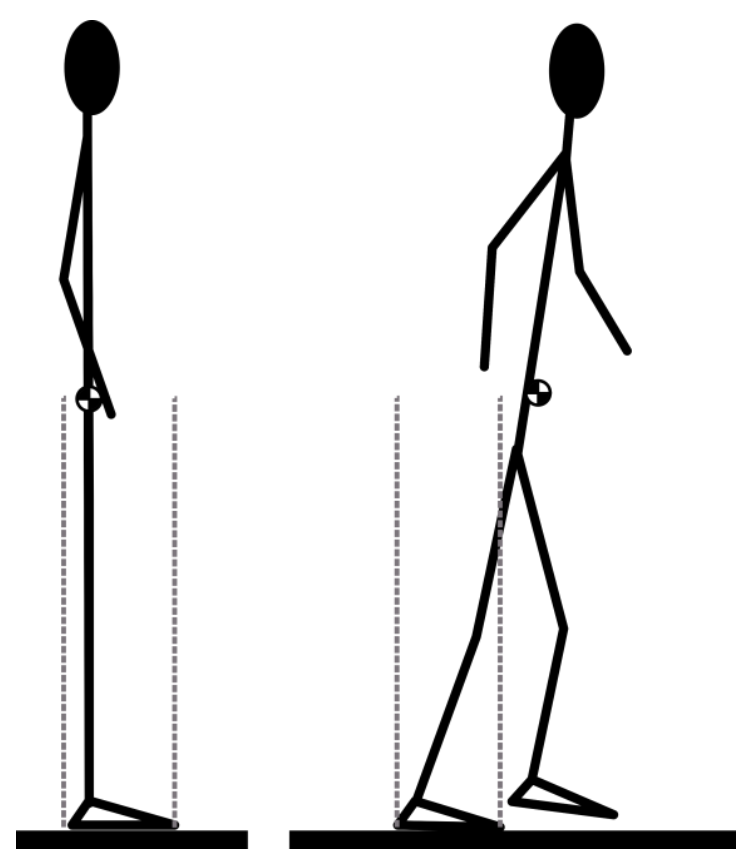

Fig. 2: Schematic of human standing and walking, denoting the relative position of the centre of mass to the base of support in the sagittal plane.

"balance"; Pollock et al., 2000). Definitions can be based on practical criteria, for example, whether a person falls in a certain environment, or they can be based on more specific criteria, such as whether or not particular components of a person's gait pattern are highly variable. Furthermore, there are different scientific definitions of a "stable" system. Stable could refer to the position of the centre of mass in relation to the base of support, whereby mechanical stability is achieved if the projection of the centre of mass to the ground is within the area within the base of support in the transverse plane, as is usually the case during quiet stance (Fig. 2). In a dynamic situation such as biped walking, the centre of mass projection will often be outside of the base of support, meaning that by this definition of stability, walking will be categorised as unstable (Winter, 1995; Fig. 2).

Walking is not static, and several researchers have proposed that the centre of mass velocity must also be accounted for in order to assess the dynamic stability of gait (Townsend, 1985, Pai and Patton, 1997, Hof et al., 2005; for a historical overview of these models, see Bruijn and van Dieën, 2018). Finally, gait stability can also refer to the linear or non-linear variability in movement during gait, which do not necessarily relate to the previous two definitions, nor to each other (Dingwell et al., 2001, Granata and England, 2007, Stergiou and Decker, 2011, Perry and Srinivasan, 2017). Given the multiple definitions of stability that can be applied to gait, simple and specific terminology are critical (Stergiou and Decker, 2011). To this end, clear requirements for what we define as "stable gait" can help guide measurement and definition. Here, the three criteria for stable gait proposed by Bruijn et al. (2013) are helpful in providing a framework: 
"...(i) the system has to be able to recover from or limit the small perturbations that occur during every stride (e.g. owing to small differences in floor height and noise), (ii) the system has to be able to recover from large perturbations, which require a change in behaviour, and (iii) the largest recoverable perturbation specified by the limits of the system needs to be larger than the perturbations encountered.” Bruijn et al. (2013)

Bruijn and colleagues' first criterion is most applicable to steady state gait with or without small perturbations that would not necessarily require large response behaviours. These could include subtle changes in the external environment (e.g. uneven ground), or internal perturbations, such as fatigue (i.e. acute internal perturbation) or neuromuscular deficits (i.e. chronic internal perturbations) that have small but meaningful effects on ongoing gait. In these situations, linear and non-linear measures assessing the quantity and structure of the variability in locomotion could provide insight (Bruijn et al., 2013). In the second and third criteria, response behaviours to large perturbations to gait are the focus, such as recovery actions following tripping or slipping. For these situations, due to the acute nature of the events, measures related to the centre of mass and base of support relationship (i.e. the mechanical stability of the body configuration) during such responses are of most use (Bruijn et al., 2013). One of the most common parameters used to assess the stability of the body configuration during dynamic movements such as gait, is the margin of stability (Hof et al., 2005, Hof, 2008). Based on an inverted pendulum model of human gait, the margin of stability is the horizontal distance between the anterior boundary of the base of support (assessed by the centre of pressure under the foot) and the extrapolated centre of mass $\left(\mathrm{X}_{\mathrm{CoM}}\right)$ calculated as:

$$
\mathrm{X}_{\mathrm{CoM}}=\mathrm{CoM}+\frac{\mathrm{V}_{\mathrm{CoM}}}{\sqrt{\frac{\mathrm{g}}{\mathrm{L}}}}
$$

where $\mathrm{CoM}$ is the horizontal position of the centre of mass, $\mathrm{V}_{\mathrm{CoM}}$ is the horizontal velocity of the centre of mass and the denominator is the eigenfrequency of the inverted pendulum, where $\mathrm{g}$ is gravitational acceleration and $\mathrm{L}$ is the length of the inverted pendulum. A margin of stability of zero represents the configuration at which the swinging inverted pendulum would come to a perfect standstill above the centre of pressure (Fig. 3). Likewise, a negative margin of stability represents a situation in which the inverted pendulum would swing beyond the centre of pressure and a positive value would lead to the inverted pendulum not reaching a vertical position (Fig. 3).

As one can see in the formula, the walking velocity affects this measure of stability, which is an issue discussed in detail in Part Three of this dissertation. In fact, when using the centre of pressure at foot contact as the anterior boundary of the base of support, self-selected walking speeds lead to negative margin of stability values for the sagittal plane, leading some researchers to question the utility of the anteroposterior margin of stability as a measure of gait stability (Bruijn et al., 2013). However, for efficient gait, it makes sense that the pendulum should fall forward after heel strike, and indeed have short periods of instability (Winter, 1995, Kuo et al., 2005, Kuo, 2007). There is a 

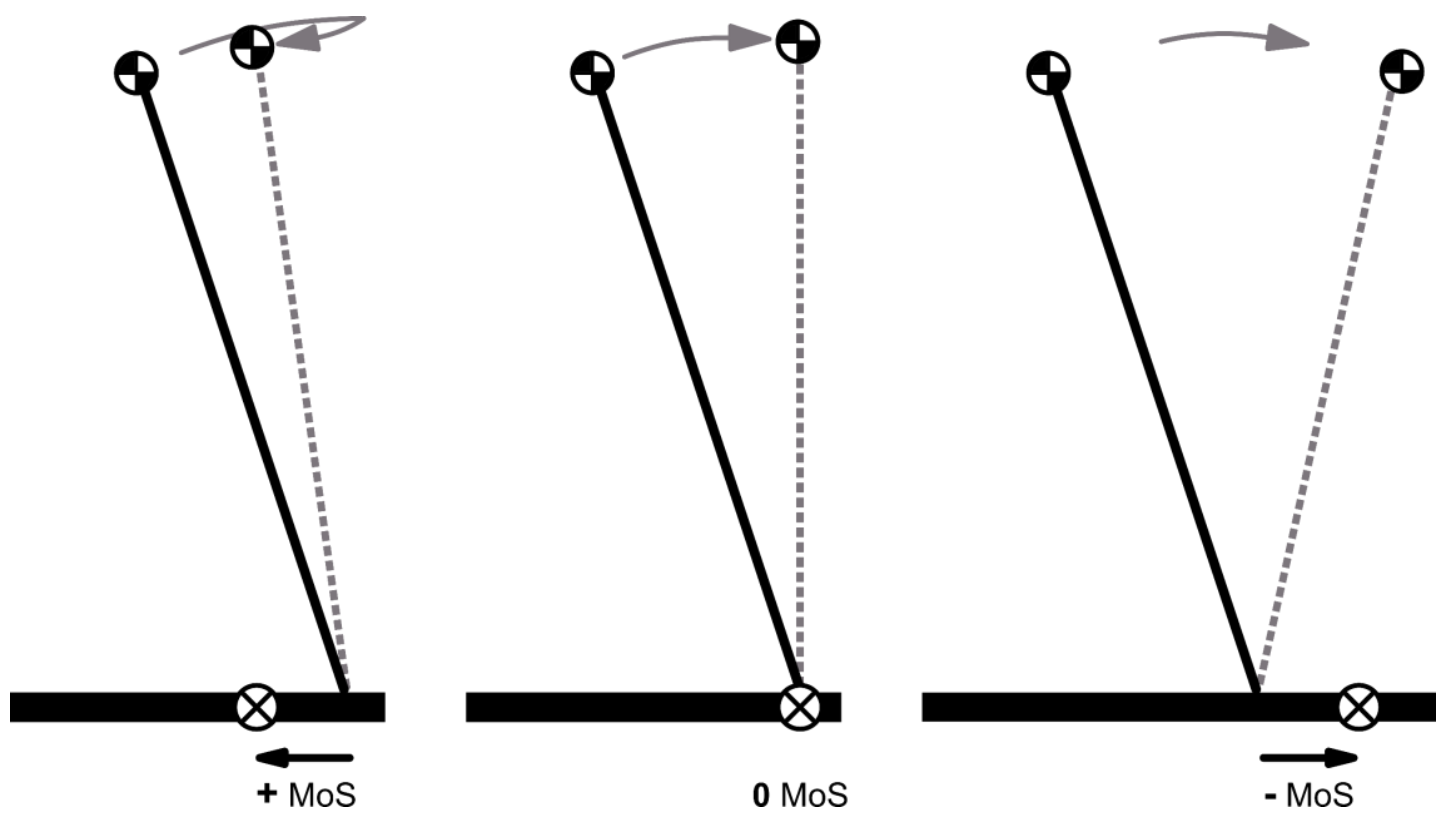

Fig. 3: Schematic illustration of the extrapolated centre of mass concept (Hof et al., 2005). Left: The horizontal position of the centre of pressure exceeds that of the extrapolated centre of mass (X), leading to a positive margin of stability (MoS) and a return in the swing of the pendulum. Centre: The horizontal position of the centre of pressure is exactly at the same as that of the extrapolated centre of mass, leading to a MoS of zero and a perfect upright standstill of the pendulum. Right: the horizontal position of the extrapolated centre of mass exceeds that of the centre of pressure, leading to a negative MoS and a continuing forward swing of the pendulum.

distinction to be made here between the stability of the inverted pendulum model at one specific time point (heel strike) and gait stability per se. As suggested by Stergiou and Decker (2011), researchers should be explicit in their definition of gait stability and should be specific with regards to what the parameters they apply actually measure. In the context of this dissertation, the margin of stability concept is applied to assess the stability of the body configuration, accounting for centre of mass velocity, at specific time points (i.e. heel strike) during gait.

Like gait stability, gait adaptability is a phrase that has been applied in many contexts, but most uses typically refer to the ability to alter gait to cope with changes in the environment. There is a slight difference between gait stability and gait adaptability in the context of gait perturbations, in that stability can be viewed as a desirable outcome of the system, whereas adaptability can be viewed as a desirable capacity or property of the system, to be able to achieve stability in different environments. It is interesting to note that the ability to adapt locomotion to meet environmental demands was proposed as an independent criterion of successful locomotion, with equilibrium control as a separate criterion by Forssberg (1982) (outlined in Patla, 1991). With this in mind, it is important to consider the general mechanisms of balance control during gait and how these might be adapted to benefit gait stability. 
Hof (2007) outlined three mechanisms of standing balance control, namely, moving the centre of pressure under the feet, counter rotation of body segments about the centre of mass and applying an external force other than the ground reaction forces (for example, by grasping a handrail). Maki and McIlroy (1997) also outlined a number of strategies for balance recovery, categorised by the nature of support, rather than mechanical characteristics, namely, fixed-support and change-in-support strategies. There is some overlap in these balance control categories, as a fixed-support strategy can incorporate both centre of pressure changes under the feet and counter rotation of body segments, and a change-in-support strategy would incorporate application of an external force other than ground reaction forces. In the context of large mechanical perturbations to gait, such as slips and trips, a change-in-support strategy involving grasping actions is only possible in specific environments, while a change-in-support strategy involving rapid corrective stepping, thereby changing the centre of pressure and increasing the base of support, may be a more common recovery action, which in itself also includes counter rotations of body segments around the centre of mass. All of the above strategies or mechanisms imply a reactive control of balance, whereby the recovery actions are performed in direct response to balance loss. However, these responses may be modulated over time in both predictive and reactive manners (Pavol et al., 2004, Bierbaum et al., 2010, Bierbaum et al., 2011). After experiencing a particular form of balance loss, a predictive alteration in gait can be made to help the body resist the effects of the perturbation, should it occur again, but a reactive alteration can also be made, as the response following the perturbation becomes more effective over time. Examinations of these adaptation processes are reported in Chapters 2.2, 4.1 and 4.2 of this dissertation. 


\section{7: Aims and Outline of the Dissertation}

Falls among older adults are a common and increasing problem. Falls due to mechanical perturbations during walking present a significant threat to the health, mobility and wellbeing of older adults. As such, a more detailed understanding of the stability and adaptation of gait during such mechanical perturbations may help improve the effectiveness of interventions for falls reduction, by identifying specific mechanisms of gait adaptation that can be exploited. The aim of this dissertation is to further our understanding of how old age might affect gait stability and adaptability, with the perspective that this knowledge could improve the effectiveness and specificity of exercisebased falls reduction interventions for both healthy and clinical populations at an increased risk of falls.

In Part Two of this dissertation, an examination of gait variability and stability is conducted during walking tasks examining predominantly predictive control during unperturbed and perturbed gait. Chapter 2.1 uses multiple walking speeds to assess this in young and older healthy adults, as well as adults with bilateral vestibulopathy, in order to elucidate the potential role of the vestibular system in this control. Chapter 2.2 uses a sustained resistance perturbation to explore the predictive adaptation of gait to control stability in young, middle-aged and older adults, to investigate how such control might change across the adult lifespan.

Part Three of this dissertation deals with methodological aspects of examining reactive gait stability using perturbations and how we might improve our experimental procedures to better understand this concept. Chapter 3.1 reviews past methodology for examining reactive gait stability during unexpected perturbations in older adults and makes suggestions for future research. Chapter 3.2 then acts upon some of these recommendations and presents a method to reduce interindividual differences in initial gait stability, allowing a theoretically more accurate examination of responses to gait perturbations and any subsequent adaptations in gait.

Part Four of this dissertation then applies the improved methodology in two studies on reactive gait stability. Chapter 4.1 examines how young humans adapt, retain and transfer adaptations in gait stability to controlled gait perturbations. Chapter 4.2 then examines if the processes of adaptation and transfer are altered in older adults and if the methodology developed and applied in this dissertation leads to different conclusions than those previously reported in the literature regarding age-related differences in gait stability.

Part Five of this dissertation reports a review of the literature on how such perturbations as used in this dissertation have been applied in research on falls prevention and presents a critical discussion of factors relating to both effectiveness and feasibility of perturbation-based balance training in clinical practice.

Finally, Part Six of this dissertation will synthesise and discuss the results of all parts of this dissertation, discuss broader issues around task specific exercise-based falls prevention and the overall conclusions of this dissertation will be presented. 


\section{Part Two:}

\section{Unperturbed and Predictive Gait Stability with Ageing and Vestibulopathy}

“..it should come as no surprise that any condition that impairs sensory or muscle function may impair gait stability due to an impaired ability to control foot placement, as becomes apparent with ageing and pathology."

- Bruijn \& van Dieën (2018) 


\section{1:}

\section{The walking speed-dependency of gait variability in bilateral}

vestibulopathy and its association with clinical tests of vestibular function

Christopher McCrum, Florence Lucieer, Raymond van de Berg, Paul Willems, Angélica Pérez Fornos, Nils Guinand, Kiros Karamanidis, Herman Kingma, Kenneth Meijer

Submitted. Available in Preprint form as:

MCCRUM, C., LUCIEER, F., VAN DE BERG, R., WILLEMS, P., PÉREZ FORNOS, A., GUINAND, N., KARAMANIDIS, K., KINGMA, H. \& MEIJER, K. 2018. Is faster always better? The walking speeddependency of gait variability in bilateral vestibulopathy. bioRxiv. doi: 10.1101/413955

(C) The Authors 2018. This work is licensed under the Creative Commons Attribution 4.0 International License. http://creativecommons.org/licenses/by/4.0/ 


\begin{abstract}
Understanding balance and gait deficits in vestibulopathy may help improve clinical care and our knowledge of the vestibular contributions to balance. Here, we examined walking speed effects on gait variability in healthy adults and in adults with bilateral vestibulopathy (BVP). Forty-four people with BVP, 12 healthy young adults and 12 healthy older adults walked at $0.4 \mathrm{~m} / \mathrm{s}$ to $1.6 \mathrm{~m} / \mathrm{s}$ in $0.2 \mathrm{~m} / \mathrm{s}$ increments on a dual belt, instrumented treadmill. Using motion capture and kinematic data, the means and coefficients of variation for step length, time, width and double support time were calculated. The BVP group also completed a video head impulse test and examinations of ocular and cervical vestibular evoked myogenic potentials and dynamic visual acuity. Walking speed significantly affected all gait parameters. Step length variability at slower speeds and step width variability at faster speeds were the most distinguishing parameters between the healthy participants and people with BVP, and among people with BVP with different locomotor capacities. Step width variability, specifically, indicated an apparent persistent importance of vestibular function at increasing speeds. Gait variability was not associated with the clinical vestibular tests. Our results indicate that gait variability at multiple walking speeds has potential as an assessment tool for vestibular interventions.
\end{abstract}




\section{Introduction}

Ever since a chance observation of a dog with acute unilateral vestibulopathy who demonstrated less imbalance during running than during walking (Brandt et al., 1999), the interactions of gait velocity, imbalance and vestibular symptoms in people with vestibulopathy have become a topic of great interest. Inspired by the observation in the dog, Brandt et al. (1999) demonstrated with a simple setup that humans with acute unilateral vestibulopathy could run with less deviation to the affected side than while walking. Since then, three studies have reported reductions in temporal gait variability and reductions in stride length variability in bilateral vestibulopathy (BVP) during faster, compared to slower walking (Schniepp et al., 2012, Wuehr et al., 2016, Schniepp et al., 2017). BVP, a severe bilateral reduction of vestibular function that results in severe balance deficits and an increased risk of falls (Guinand et al., 2012a, van de Berg et al., 2015, Horak et al., 2016, Lucieer et al., 2016, Schlick et al., 2016, Sprenger et al., 2017), was recently defined by the Bárány Society (Strupp et al., 2017) and represents one of the most debilitating vestibular disorders. Interestingly, the same studies revealed that patients with BVP do not self-select walking speeds that minimize temporal or spatial gait variability (Schniepp et al., 2012, Wuehr et al., 2016, Schniepp et al., 2017), which may suggest that these are not the only source of instability or inefficiency with which people with BVP must cope. However, further research into the relationships between vestibulopathy, walking speed and gait variability is needed to confirm and expand on these previous findings, as these three previous studies had some potential drawbacks, namely a limited number of gait parameters being analysed (Schniepp et al., 2012), too few strides (Owings and Grabiner, 2003, Hollman et al., 2010, Konig et al., 2014, Riva et al., 2014) for a robust analysis of gait variability (Schniepp et al., 2012, Schniepp et al., 2017), the use of only preferred walking speeds or percentages of preferred walking speeds (ecologically valid, but less control over influencing factors) (Schniepp et al., 2012, Wuehr et al., 2016, Schniepp et al., 2017), small sample size (Wuehr et al., 2016), lack of a healthy control group (Wuehr et al., 2016, Schniepp et al., 2017) and the presence of sham vestibular stimulation in the control condition (Wuehr et al., 2016). The study of the severe balance and gait deficits in people with BVP is both important for improving clinical care and for objective quantification of the effects of novel interventions, such as vestibular implants (Guyot et al., 2016, Lewis, 2016). Furthermore, it is fundamental to our understanding of the vestibular contributions to gait and balance control.

The sensory contributions to gait appear to depend on walking speed, which may partly explain the above described findings and will affect walking speed selection in people with vestibulopathy. Visual perturbations such as distorting prisms or closed eyes have reducing impact on most gait variability parameters as one walks faster (Jahn et al., 2001, Wuehr et al., 2013) with the exception of step width variability, which appears to increase with visual perturbation at faster walking speeds (Wuehr et al., 2013). Similarly, vestibular perturbations via galvanic vestibular stimulation have less impact on gait direction and variability at higher speeds (Fitzpatrick et al., 1999, Jahn et al., 2000). Additionally, the vestibular influence on lower limb muscles (determined by examining vestibulo-muscular coupling via lower limb muscle electromyography during vestibular stimulation) is selectively suppressed with increased cadence and speed during walking 
(Dakin et al., 2013, Forbes et al., 2017), purported to be related to a shift in the control mechanisms of mediolateral stability with increasing walking speeds from active stabilization at the lower limb joints during the stance phase to foot placement (Bauby and Kuo, 2000, Dakin et al., 2013). Despite selective suppression of the vestibular influence on some lower limb muscles at faster walking speeds, significant increases in frontal spatial variability with increasing walking speeds have been reported in BVP (Wuehr et al., 2016), suggesting that vestibular information remains important for mediolateral stability during gait at faster speeds.

To further investigate the walking speed dependency of gait variability in vestibulopathy, we analyzed the gait of people with BVP and of healthy control participants. We aimed to determine the effects of systematic increases in walking speed on spatiotemporal gait parameters and their variability in these participant groups. Secondly, we aimed to assess if these parameters would differentiate between healthy participants, and participants with BVP who could and could not complete all of the planned walking speed trials. We hypothesized that, for all participants, step and double support time and step length variability would systematically reduce with increases in walking speed, whereas step width variability would systematically increase, in agreement with previous work (Schniepp et al., 2012, Wuehr et al., 2016, Schniepp et al., 2017). We further postulated that, based on earlier studies and despite their limitations described above (Schniepp et al., 2012, Schniepp et al., 2017), step and double support time and step length variability at slower walking speeds would be most distinguishing between the healthy control participants and patients with BVP, and also between the patients with BVP that could completely and only partly complete the measurement protocol, whereas step width variability would be most distinguishing at faster walking speeds, based on one study showing an increase in BVP (Wuehr et al., 2016). Additionally, we conducted an explorative analysis in the patient groups examining correlations between the outcomes of the most distinguishing gait parameters identified and clinical vestibular tests conducted as part of a larger clinical study (video head impulse test [vHIT], ocular and cervical vestibular evoked myogenic potentials [oVEMP and cVEMP] and dynamic visual acuity [DVA]) that are indicative of vestibular functional integrity and commonly used in clinical settings, with the aim to explore if these tests could give an indication about gait-related function in BVP.

\section{Methods}

\section{Participants}

Forty-four people with BVP participated in this study (22 males, 22 females; age: $57.6 \pm 11.5$ years, age range: 21 to 74 ; height: $174.5 \pm 9.7 \mathrm{~cm}$; weight: $80.4 \pm 17 \mathrm{~kg})$. Inclusion criteria were a prior diagnosis of bilateral vestibular hypofunction at the Maastricht University Medical Centre+ (imbalance and/or oscillopsia during locomotion and summated slow phase mean peak velocity of the nystagmus of less than $20 \%$ s during bithermal caloric tests) and the self-reported ability to walk independently without assistance. Please note that this study began prior to the publication of the Bárány Society guidelines (Strupp et al., 2017), which are slightly different. Potential participants were 
not included if they were unable or unwilling to stop taking anxiety or depression medication for the week before the measurements. In addition, two healthy control groups comprised of 12 healthy younger adults (Young; 5 males, 7 females; 25.1 \pm 2.8 years; $174.9 \pm 7.3 \mathrm{~cm} ; 72.6 \pm 13.5 \mathrm{~kg}$ ) and 12 healthy older adults (Older; 8 males, 4 females; $71.5 \pm 4.8$ years; $171.5 \pm 9.1 \mathrm{~cm} ; 79.5 \pm 11.8 \mathrm{~kg}$ ) with no history of balance or gait difficulties and no history of dizziness participated in this study. These specific groups were included to account for the age range in the BVP group and to provide an estimation of the effect of ageing alone on the outcome parameters. The study was explained before obtaining written informed consent, was conducted in accordance with the Declaration of Helsinki and was approved by the Maastricht University Medical Centre medical ethics committee (gait measurements: NL58205.068.16; vestibular tests: NL52768.068.15).

\section{Gait Analysis Setup, Data Processing and Procedure}

The gait measurements were conducted using the Computer Assisted Rehabilitation Environment Extended (CAREN; Motekforce Link, Amsterdam, The Netherlands), which includes a dual-belt force plate-instrumented treadmill (Motekforce Link, Amsterdam, The Netherlands; $1000 \mathrm{~Hz})$, a 12 camera motion capture system $(100 \mathrm{~Hz}$; Vicon Motion Systems, Oxford, UK) and a virtual environment (city-style street with passing objects and structures) projected onto a 180 degrees curved screen (note that our intention was to provide optic flow for all participants, but after the first few measurements with the BVP group, it became clear that optic flow should be turned off for this group to prevent dizziness and nausea. The implications for this on the results are discussed in the limitations section). For all measurement sessions, a safety harness connected to an overhead frame was used. At the request of some of the participants with BVP, a handrail was also positioned on the treadmill, the use of which was monitored and recorded. Six retroreflective markers were attached to anatomical landmarks $(\mathrm{C} 7$, sacrum, left and right trochanter and left and right hallux) and were tracked by the motion capture system. Marker tracks were filtered using a low pass second order Butterworth filter (zero-phase) with a $12 \mathrm{~Hz}$ cut-off frequency. Foot touchdown was determined using combined force plate (50N threshold) and foot marker data (Zeni et al., 2008). This combined method was used to be able to accurately account for foot touchdowns and toe-offs occurring in the center of the treadmill triggering both force plates simultaneously. For these steps, the foot marker method was used and then corrected based on the average discrepancy between the force plate method and the marker method timing for all steps that contacted only one force plate. The spatiotemporal gait parameters of interest were step length (anteroposterior distance between the hallux markers at foot touchdown), step time (time from touchdown of one foot to touchdown of the next foot), step width (mediolateral distance between the hallux markers at foot touchdown) and double support time (time spent with both feet on the ground). Means, standard deviations and coefficients of variation (CV) were determined for each speed for each participant.

Each session began with walking familiarization trials at $0.4 \mathrm{~m} / \mathrm{s}$ up to $1.6 \mathrm{~m} / \mathrm{s}$ in $0.2 \mathrm{~m} / \mathrm{s}$ increments. At least $60 \mathrm{~s}$ were used for each speed, and further time was provided to familiarize to each speed if deemed necessary by either the participant, the CAREN 
operator or the research clinician. At the end of each speed trial, the decision to continue to the next (faster) speed was made in a similar manner. If the participant was not comfortable progressing to the next speed or if the CAREN operator or research clinician did not think it was safe or feasible to progress, then the participant continued at the current speed instead. Participants were then given sufficient rest before continuing with the measurements. Single two-to-three-minute-long measurements (to ensure a minimum of 60 strides per speed) were then conducted at each prescribed speed that was completed during familiarization. Multiple set walking speeds were used as opposed to the majority of previous studies which have used either percentages of preferred walking speeds or selfperceived slow, normal and fast walking speeds, in order to have more control over the walking speed condition.

\section{Clinical Vestibular Function Tests Setup and Procedures}

Following a sufficient rest period that was determined on an individual basis, the BVP group proceeded with the clinical vestibular testing battery. Between each test, sufficient rest was provided based on feedback from the patient and the judgement of the clinical researcher. The vHIT was performed with the EyeSeeCam system (EyeSeeCam VOG; Munich, Germany) and the ICS Impulse system (GN Otometrics A/S, Denmark) to test semicircular canal function and determine the gain of the vestibulo-ocular reflex (VOR). Both systems measured the movement of the right eye. The distance of the back of the static chair was 2 meters to the point of fixation. The point of fixation consisted of a green dot on the wall, produced by a laser on a tripod. If necessary, adhesive plasters were used to lift the upper eyelid a little to secure the visibility of the pupil for the camera in all directions. Goggle movement was minimized by adjusting the strap of the goggles to every subject. The vHIT system was calibrated according to the protocol of the system. After calibration, the subject was instructed to not touch their head including the goggles. The examiner stood behind the participant with two hands firmly on top of the participant's head without touching the strap of the goggles. The examiner then applied head impulses in six different movements to test each canal (McGarvie et al., 2015). The horizontal head impulses comprised a peak velocity of $>150 \%$ s and the vertical head impulses a peak head velocity of $>100^{\circ} / \mathrm{s}$. The amplitude of the movements was $10-20^{\circ}$. Only outward impulses were used (van Dooren et al., 2018). The vHIT was defined as abnormal if the VOR gain was below 0,7 and/or if covert saccades were observed in $50 \%$ or more of the traces (McGarvie et al., 2015, Yip et al., 2016).

DVA, which is used to assess gaze stabilization via the VOR during gait-related head movements, was assessed on a regular treadmill (1210 model, SportsArt, Inc., Tainan, Taiwan, China.) with the participant positioned 2.8 meters from a computer screen. Firstly, the static visual acuity was determined during stance, followed by the assessment of the DVA during walking at 2,4 and $6 \mathrm{~km} / \mathrm{h}$. One letter at a time was randomly displayed on the screen from a chart of Sloan letters (CDHKNORSVZ) (Sloan, 1959). Starting at a logMAR (log of the Minimum Angle of Resolution; (Bailey and Lovie, 1976)) of 1.0, five random letters were shown at each logMAR (decreasing in steps of 0.1 $\operatorname{logMAR}$ ). When four out of five letters were correctly identified, the corresponding logMAR 
was considered achieved. The outcome of the DVA was the difference between the static $\log$ MAR and the logMAR for each of the three walking speeds. The result was omitted if the subject needed a handrail to walk at that speed or if it wasn't possible to walk at that speed at all (Guinand et al., 2012b).

cVEMP and oVEMP were assessed with the Neuro-Audio system (v2010, Neurosoft, Ivanovo, Russia) in order to determine the function of the otolith organs (saccule and utricle, respectively) and their corresponding nerves. A monaural stimulation with in-ear earphones was used with air conduction tone bursts at $500 \mathrm{~Hz}$ and a stimulation rate of $13 \mathrm{~Hz}$ using a blackman window function with a two-cycle rise/fall and no plateau phase. Tone bursts of maximum $130 \mathrm{~dB}$ sound pressure level (SPL) were used. A stepwise approach was used to determine the threshold with a precision of $5 \mathrm{~dB}$ SPL (van Tilburg et al., 2016). Positive (P1) and negative (N1) peaks in the recorded biphasic waveform were marked for both cVEMPs and oVEMPs. The thresholds were determined as the lowest stimulus intensities to elicit recognizable peaks. If it wasn't possible to find a VEMP response, it was defined as a threshold of $>130 \mathrm{~dB}$ SPL. For the cVEMP, the participant was positioned lying down with the back positioned at a $30^{\circ}$ angle above the horizontal plane and was asked to turn their head towards the non-measured side and lift their head during the measurement. The cVEMP was recorded at the ipsilateral sternocleidomastoid muscle. Two electrodes were placed on the sternocleidomastoid muscles, the reference electrode on the sternum, and the earth electrode on the forehead. Electrode impedances of $5 \mathrm{k} \Omega$ or lower were accepted and otherwise the electrode was replaced. To ensure correct muscle contraction, a feedback system using a screen was provided. An average of 200 EMG traces with a minimum mean rectified voltage (MRV) of $65 \mu \mathrm{V}$ and a maximum MRV of $205 \mu \mathrm{V}$ was accepted (Brantberg and Lofqvist, 2007, Fujimoto et al., 2009). The oVEMP was recorded at the contralateral inferior oblique muscle. Five electrodes were used: the recording electrodes beneath the eyelid, just lateral of the pupil when gazing forward and centrally, the reference electrodes beneath the recording electrode and the earth electrode on the forehead. The participant was asked to keep their gaze at a focus point placed at a 30 degrees angle behind the head. An average of at least 300 EMG traces was accepted (Govender et al., 2011, Piker et al., 2013, Valko et al., 2016).

\section{Statistics}

From the 44 participants with BVP that started the study, 38 participants were able to complete at least the three slowest walking speeds without assistance (group hereafter referred to as BVP) and these participants' data were taken for the comparison with the healthy groups. For the within BVP comparisons, three groups were formed. One group was able to complete all of the gait measurements without assistance (BVP All Gait; $n=26$ ), the second was only able to complete some of the speeds without assistance (BVP Part Gait; $\mathrm{n}=12$; all of this group were able to complete the measurements at least up to $0.8 \mathrm{~m} / \mathrm{s}$ ) and the final group (BVP No Gait; $n=6$ ) did not start the recorded gait trials (see "Results" for details on this group). 
To investigate the walking speed effects on gait and this effect's potential interaction with vestibular function, mixed-effects models using the restricted maximum likelihood method with the fixed effects walking speed, participant group, and speed by group interaction were conducted for the means and CVs of step time and length, step width and double support time. To further investigate the potential of gait variability to distinguish between BVP groups, mixed-effects models as described above were applied with groups BVP All Gait and BVP Part Gait to the CV of all four gait parameters across all speeds that included data points from each group. Bonferroni post hoc comparisons were performed to assess the group differences within speeds for each of the gait parameters.

The vHIT testing revealed abnormal canal function in all or most directions for almost all of the participants with BVP (i.e. exceptions were two participants with BVP who had only one abnormal result out of six). As almost all outcomes were abnormal and there was no possibility to distinguish between groups, analysis of the vHIT results in relation to gait was not taken further. For all completed DVA trials with a logMAR change value during the three walking speeds compared to standing and when oVEMP or cVEMP thresholds were detected, these values were grouped and Pearson correlations with the gait parameters that showed highest variability and/or distinguished between BVP groups were conducted (see Results). Age, height, weight and body mass index (BMI) were compared across the participant groups BVP, Young and Older, and within the three BVP groups (BVP All Gait, BVP Part Gait, BVP No Gait) using one-way ANOVAs with Bonferroni corrections for multiple comparisons.

\section{Results}

Twenty-six participants with BVP were able to complete all of the gait measurements without assistance (BVP All Gait). Twelve participants with BVP were only able to complete some of the speeds (BVP Part Gait), of which one participant stopped after $0.8 \mathrm{~m} / \mathrm{s}$, one after $1.0 \mathrm{~m} / \mathrm{s}$, four after $1.2 \mathrm{~m} / \mathrm{s}$ and six after $1.4 \mathrm{~m} / \mathrm{s}$. Six participants with BVP were assigned to the BVP No Gait group for the following reasons: one participant became dizzy and nauseated during familiarization and could not continue; three participants were not able to walk during familiarization without handrail support; two participants found treadmill walking too challenging and could not continue. The demographic data of these three groups, as well as the healthy control group can be found in Table 1 . The oneway ANOVAs revealed a significant group effect (BVP, Young, Older) for age $(\mathrm{F}(2,59)=$ 88), $\mathrm{P}<0.0001)$, with age significantly differing between each of the groups $(\mathrm{P}<0.0001)$. Height, weight and BMI did not significantly differ across these groups. No significant differences in demographics were found with the three BVP groups.

The mixed-effects models with walking speed $(0.4$ to $1.6 \mathrm{~m} / \mathrm{s})$ and group (BVP, Young, Older) as factors revealed significant walking speed effects for the means and CV of step time and length, step width and double support time $(P \leq 0.0003)$, significant group effects for all parameters except step width means $(P \leq 0.0151)$ and significant walking speed by group interactions for the means of step time, double support time and step width $(P \leq 0.0053)$ and the $\mathrm{CV}$ of step width $(P<0.0001)$. The mixed-effects model results and 
Table 1. Participant Group Characteristics

\begin{tabular}{llllll}
\hline \multicolumn{1}{c}{ n } & Age (y) & Height (cm) & Weight (kg) & Body Mass Index \\
\hline Young & 12 (7 female) & $25.1 \pm 2.8^{*}$ & $174.9 \pm 7.3$ & $72.6 \pm 13.5$ & $23.6 \pm 2.8$ \\
Older & 12 (4 female) & $71.5 \pm 4.8^{*}$ & $171.5 \pm 9.1$ & $79.5 \pm 11.8$ & $26.9 \pm 2.2$ \\
BVP & 38 (20 female) & $56.1 \pm 11^{*}$ & $174.6 \pm 10.1$ & $80.2 \pm 17.6$ & $26.1 \pm 4.2$ \\
$\quad$ BVP All Gait & 26 (10 female) & $55.1 \pm 11.4$ & $176.8 \pm 9.9$ & $80.3 \pm 17.8$ & $25.4 \pm 3.8$ \\
$\quad$ BVP Part Gait & 12 (10 female) & $59.2 \pm 9$ & $169.7 \pm 9$ & $79.9 \pm 18$ & $27.6 \pm 4.7$ \\
$\quad$ BVP No Gait & 6 (2 female) & $65.3 \pm 13.6$ & $174 \pm 6.9$ & $82.4 \pm 13.4$ & $27.2 \pm 3.8$ \\
\hline
\end{tabular}

Values are means $\pm \mathrm{SD}$. *: Significantly different from each other $(P<0.0001)$.

summary of the between group Bonferroni comparisons are displayed in Fig. 1 (means) and Fig. 2 (CVs), and the full Bonferroni comparison results are available in Supplementary Tables 1 and 2 .

The mixed-effects models with walking speed $(0.4$ to $1.4 \mathrm{~m} / \mathrm{s})$ and group (BVP All Gait and BVP Part Gait) as factors revealed significant walking speed effects for the CV of all parameters $(P<0.0001)$. Significant group effects were found for the $\mathrm{CV}$ of step time, step length and double support time $(P \leq 0.0162)$ and a significant walking speed by group interaction was found for the $\mathrm{CV}$ of double support time $(P=0.0172)$. The mixed-effects model results and summary of the between group Bonferroni comparisons are displayed in Fig. 3 and the full Bonferroni comparison results are available in Supplementary Table 3 .

When cVEMP and oVEMP thresholds were detected, and when a speed of the DVA was completed, these values were taken and Pearson correlations were conducted with the CVs of step time, step length and double support time at $0.4 \mathrm{~m} / \mathrm{s}$ and the CV of step width at $1.6 \mathrm{~m} / \mathrm{s}$, being the speeds with the highest variability in those parameters from the previous analysis. These results can be seen in Table 2. Only two significant correlation of 28 were found (change in logMAR scores during the DVA with Double Support CV at $6 \mathrm{~km} / \mathrm{h}$ and oVEMP Left and Step Length CV at $0.4 \mathrm{~m} / \mathrm{s}$; Table 2 ).

\section{Post-hoc Analysis of Gait Data based on VEMP Results}

In order to further investigate differences within the patient group, we conducted an analysis of the gait data of the participants with and without at least one detected VEMP threshold for the same four parameters as the correlations: the CVs of step time, step length and double support time at $0.4 \mathrm{~m} / \mathrm{s}$ and the CV of step width at $1.6 \mathrm{~m} / \mathrm{s}$. Given that all of the participants with no VEMP threshold detected also had abnormal outcomes on the vHIT for most or all of the six directions tested, the purpose of this analysis was to compare the gait of participants with and without detectable canal and otolith function. Independent samples t-tests with Welch's corrections did not reveal any significant differences between the participants with and without at least one detectable VEMP threshold $(0.0965<\mathrm{P}<0.746)$. 
Table 2: Pearson correlations between the cVEMP and oVEMP thresholds, the change in logMAR scores during each of the three DVA walking speeds and the gait parameters.

\begin{tabular}{|c|c|c|c|c|c|}
\hline & & $\begin{array}{c}\text { Step Time CV } \\
0.4 \mathrm{~m} / \mathrm{s}\end{array}$ & $\begin{array}{c}\text { Step Length CV } \\
0.4 \mathrm{~m} / \mathrm{s}\end{array}$ & $\begin{array}{l}\text { Double Support } \\
\text { Time CV 0.4m/s }\end{array}$ & $\begin{array}{c}\text { Step Width CV } \\
1.6 \mathrm{~m} / \mathrm{s}\end{array}$ \\
\hline & $\mathrm{r}$ & 0.08987 & 0.3259 & 0.2576 & -0.3501 \\
\hline cVEMP & $95 \% \mathrm{CI}$ & -0.3935 to 0.5343 & -0.1662 to 0.6881 & -0.2379 to 0.6467 & -0.7554 to 0.2489 \\
\hline \multirow{2}{*}{ Right } & P (two-tailed) & 0.7229 & 0.1868 & 0.302 & 0.241 \\
\hline & $\mathrm{n}$ & 18 & 18 & 18 & 13 \\
\hline & $\mathrm{r}$ & -0.2425 & 0.1195 & -0.1732 & -0.5043 \\
\hline cVEMP & $95 \% \mathrm{CI}$ & -0.659 to 0.2878 & -0.3999 to 0.5808 & -0.616 to 0.3528 & -0.8362 to 0.09795 \\
\hline \multirow{2}{*}{ Left } & P (two-tailed) & 0.3655 & 0.6595 & 0.5212 & 0.0945 \\
\hline & $\mathrm{n}$ & 16 & 16 & 16 & 12 \\
\hline \multirow{4}{*}{$\begin{array}{l}\text { oVEMP } \\
\text { Right }\end{array}$} & $\mathrm{r}$ & 0.4653 & 0.561 & 0.286 & 0.4649 \\
\hline & $95 \% \mathrm{CI}$ & -0.7074 to 0.9554 & -0.6361 to 0.9654 & -0.7975 to 0.9329 & -0.7076 to 0.9553 \\
\hline & P (two-tailed) & 0.4297 & 0.3251 & 0.6408 & 0.4301 \\
\hline & $\mathrm{n}$ & 5 & 5 & 5 & 5 \\
\hline \multirow{4}{*}{$\begin{array}{l}\text { oVEMP } \\
\text { Left }\end{array}$} & $\mathrm{r}$ & -0.04995 & 0.7914 & 0.08001 & -0.3605 \\
\hline & $95 \% \mathrm{CI}$ & -0.6911 to 0.6352 & 0.2684 to 0.9541 & -0.6169 to 0.7066 & -0.8494 to 0.4614 \\
\hline & P (two-tailed) & 0.8985 & 0.0111 & 0.8379 & 0.3803 \\
\hline & $\mathrm{n}$ & 9 & 9 & 9 & 8 \\
\hline \multirow{4}{*}{$\begin{array}{l}\text { DVA } \\
2 \mathrm{~km} / \mathrm{h}\end{array}$} & $\mathrm{r}$ & -0.1244 & 0.01669 & -0.2151 & -0.09623 \\
\hline & $95 \% \mathrm{CI}$ & -0.4271 to 0.2034 & -0.3046 to 0.3346 & -0.5004 to 0.1123 & -0.4662 to 0.3024 \\
\hline & P (two-tailed) & 0.4568 & 0.9208 & 0.1947 & 0.6401 \\
\hline & $\mathrm{n}$ & 38 & 38 & 38 & 26 \\
\hline \multirow{4}{*}{$\begin{array}{l}\text { DVA } \\
4 \mathrm{~km} / \mathrm{h}\end{array}$} & $\mathrm{r}$ & 0.06088 & -0.1711 & 0.03413 & 0.2422 \\
\hline & $95 \% \mathrm{CI}$ & -0.2639 to 0.3733 & -0.4654 to 0.1572 & -0.2887 to 0.35 & -0.1602 to 0.5756 \\
\hline & $\mathrm{P}$ (two-tailed) & 0.7166 & 0.3043 & 0.8388 & 0.2332 \\
\hline & $\mathrm{n}$ & 38 & 38 & 38 & 26 \\
\hline \multirow{4}{*}{$\begin{array}{l}\text { DVA } \\
6 \mathrm{~km} / \mathrm{h}\end{array}$} & $\bar{r}$ & -0.3145 & -0.3199 & -0.4338 & -0.06129 \\
\hline & $95 \% \mathrm{CI}$ & -0.6371 to 0.1018 & -0.6406 to 0.09588 & -0.7125 to -0.0369 & -0.4803 to 0.3805 \\
\hline & $\mathrm{P}$ (two-tailed) & 0.1345 & 0.1275 & 0.0342 & 0.7918 \\
\hline & $\mathrm{n}$ & 24 & 24 & 24 & 21 \\
\hline
\end{tabular}




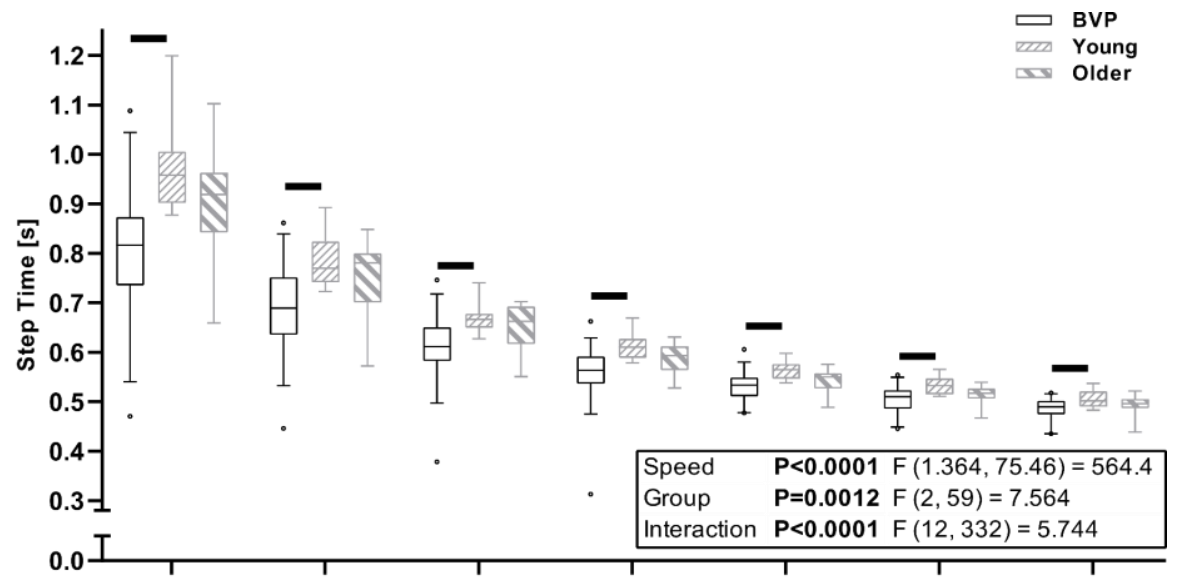

Fig. 1: Boxplots of the median, interquartile range and $5^{\text {th }}$ and $95^{\text {th }}$ percentile of the means of step time, step length, double support time and step width across all conducted walking speeds in BVP, Young and Older participant groups. The black horizontal lines indicate

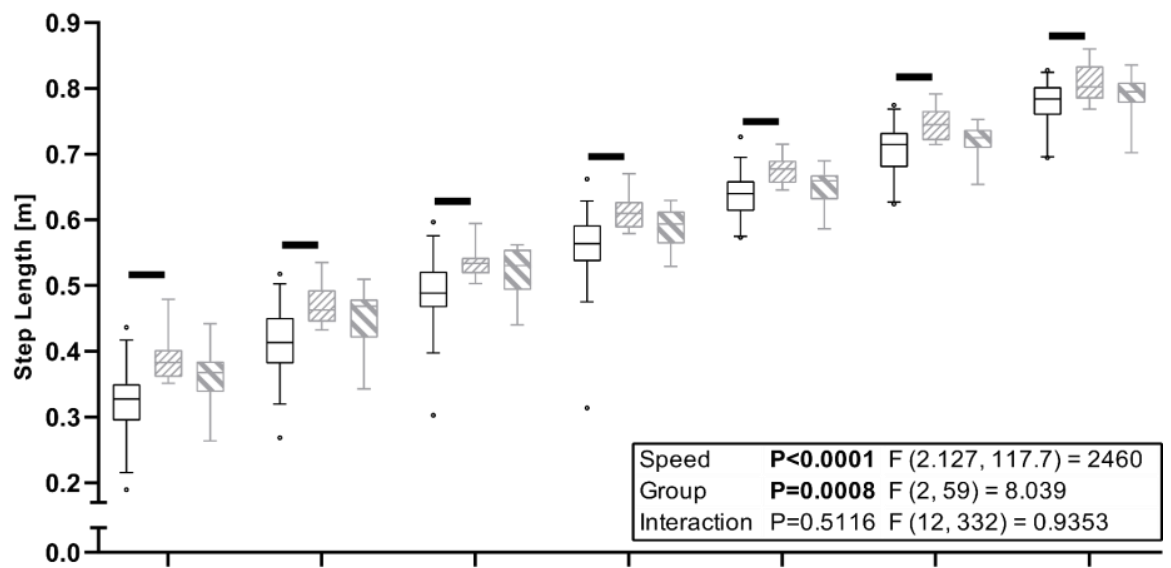
significant between group differences for the indicated speed $(\mathrm{P}<0.05$, Bonferroni adjusted).
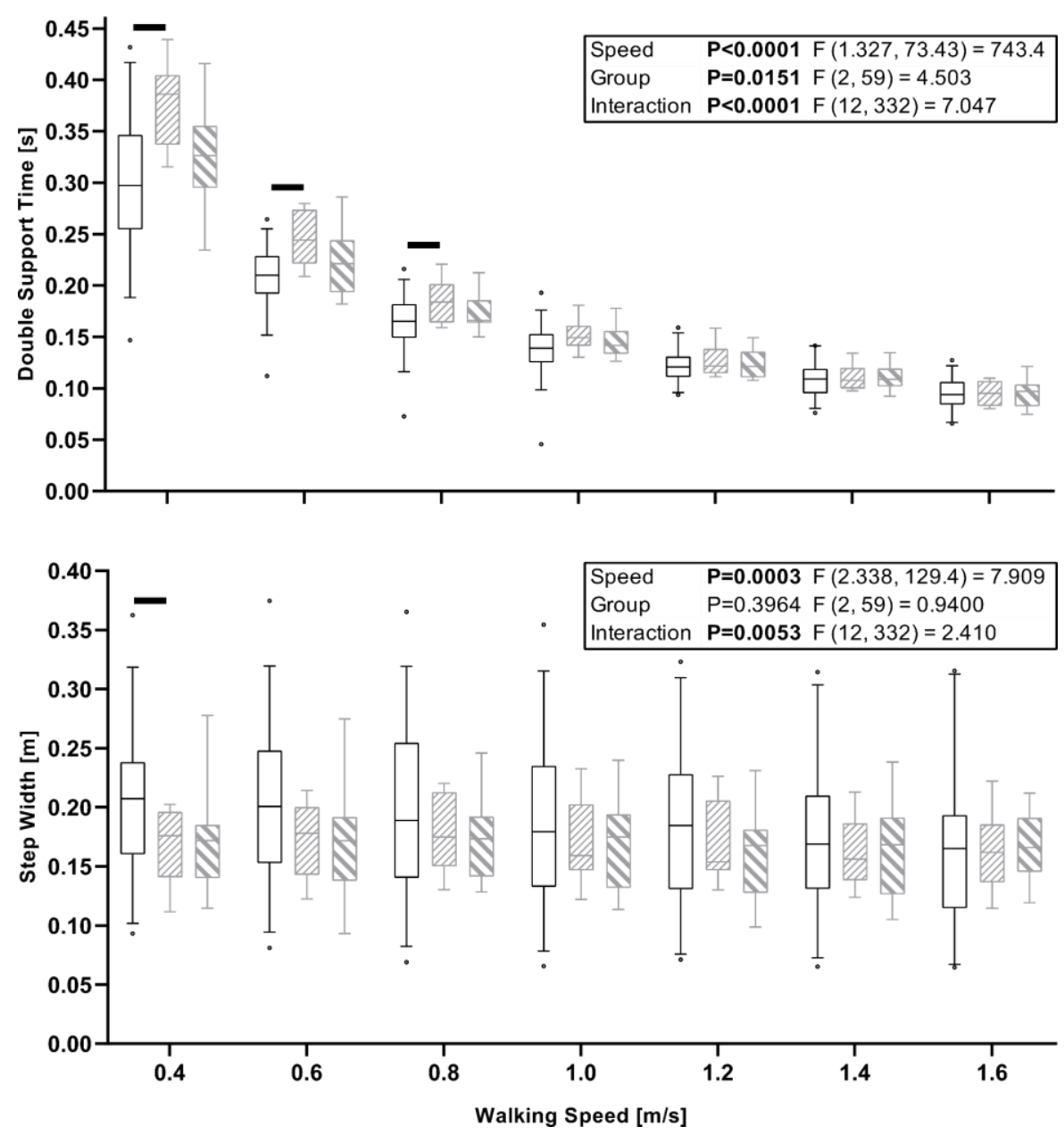

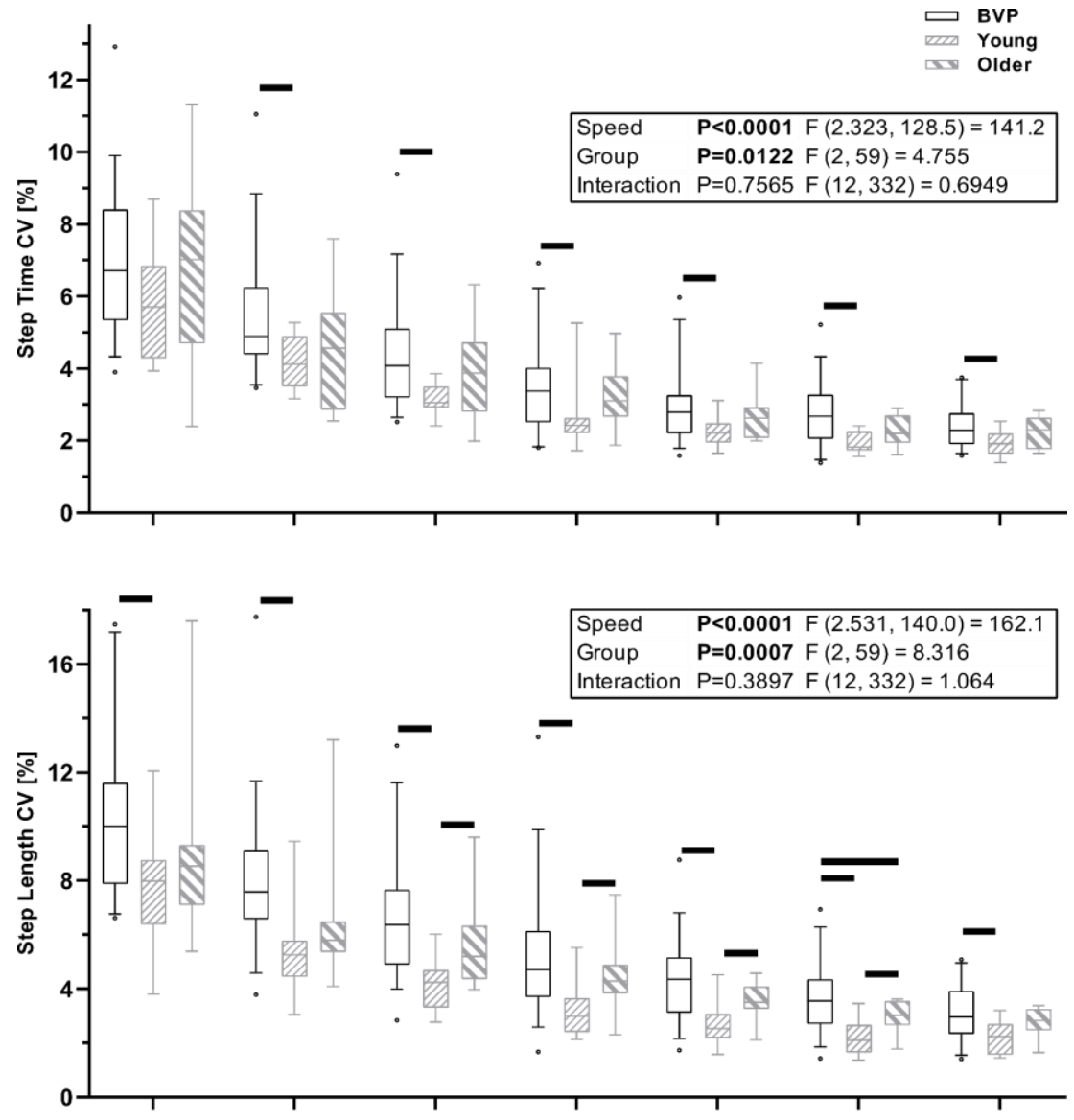

Fig. 2: Boxplots of the median, interquartile range and $5^{\text {th }}$ and $95^{\text {th }}$ percentile of the coefficients of variation $(\mathrm{CV})$ of step time, step length, double support time and step width across all conducted walking speeds in BVP, Young and Older participant groups. The black horizontal lines indicate significant between group differences for the indicated speed $(\mathrm{P}<0.05$, Bonferroni adjusted).
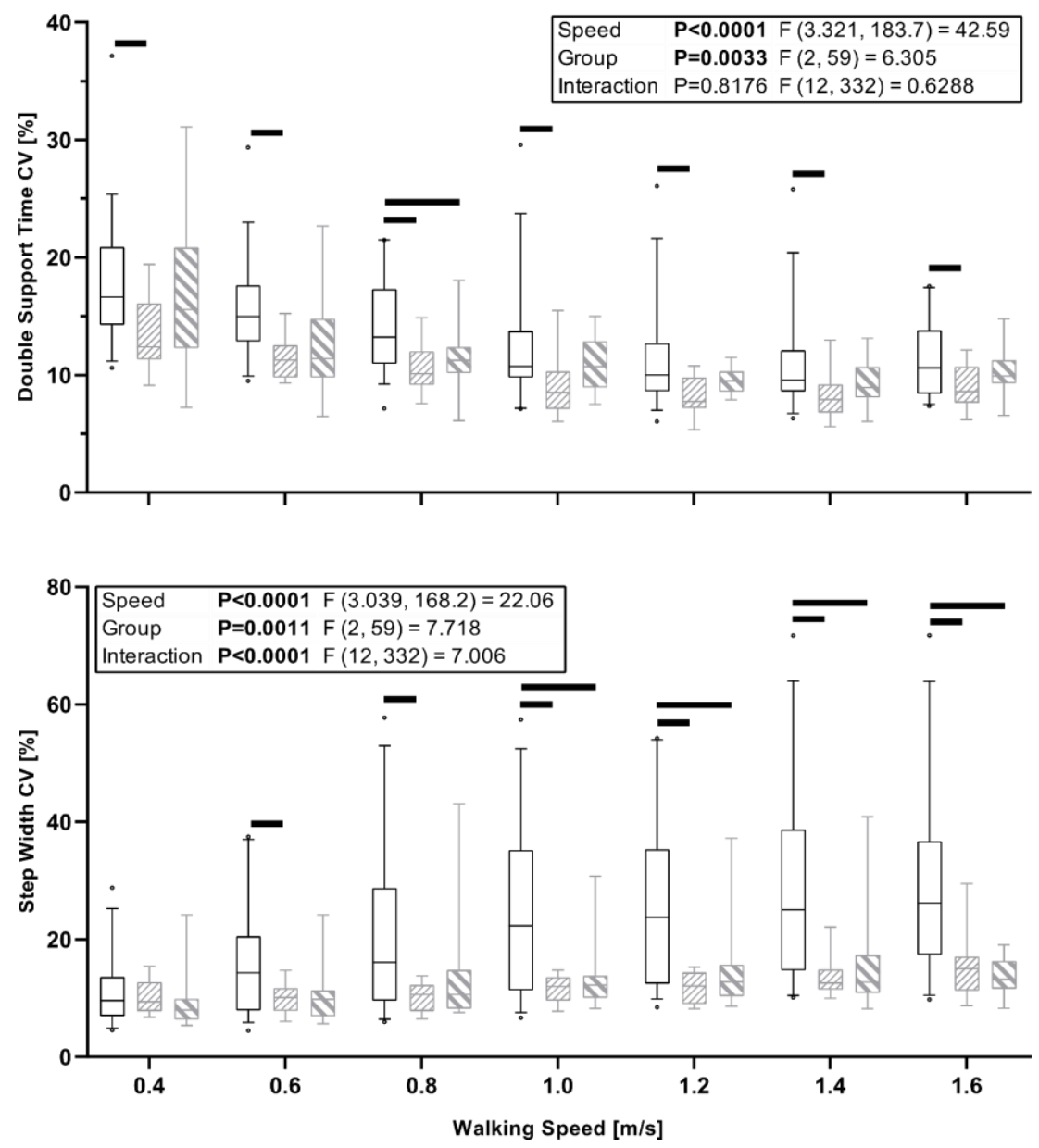

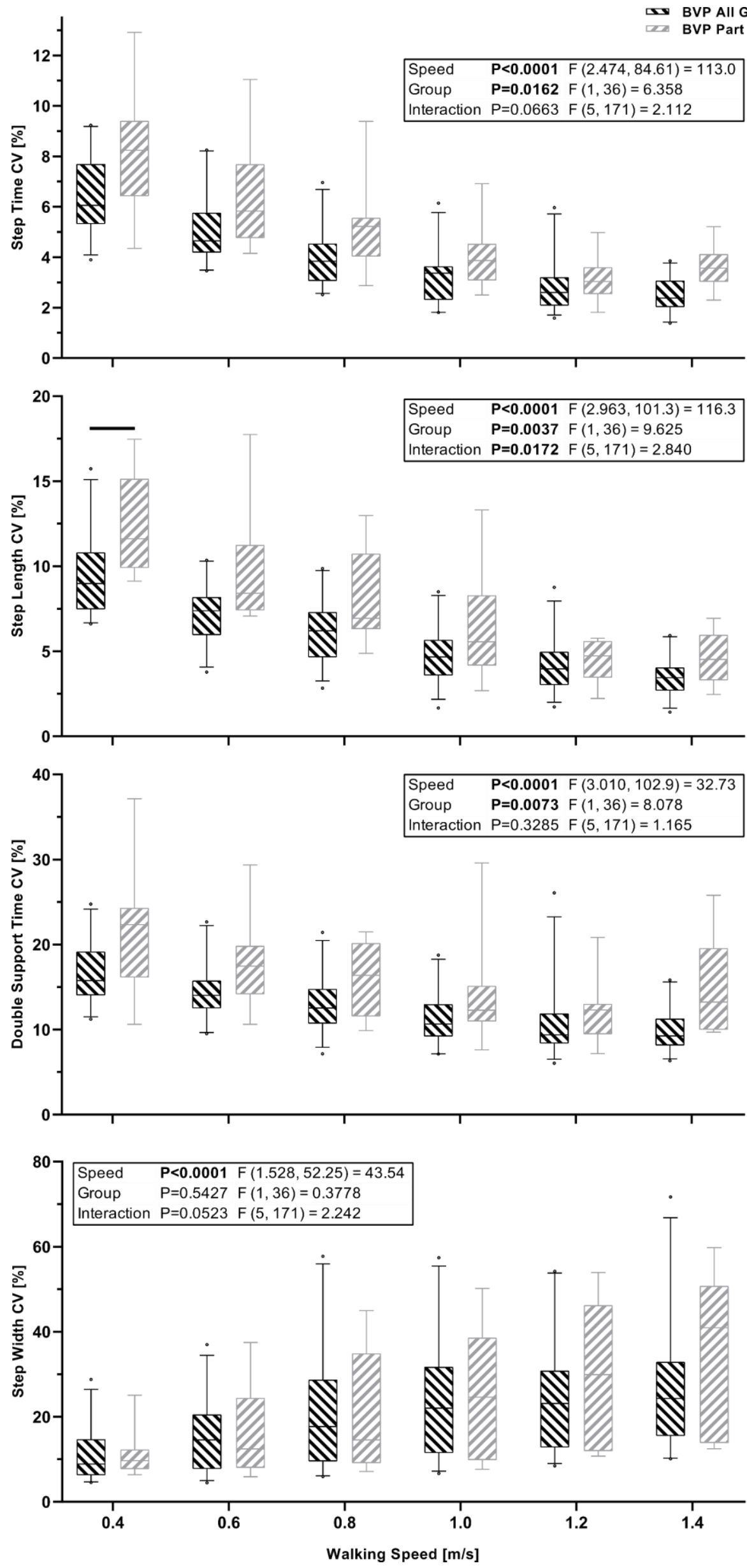

Fig. 3: Boxplots of the median, interquartile range and $5^{\text {th }}$ and $95^{\text {th }}$ percentile of the coefficients of variation (CV) of step time, step length, double support time and step width across all walking speeds with data from participant groups BVP All Gait and BVP Part Gait. The black horizontal lines indicate significant between group differences for the indicated speed $(\mathrm{P}<0.05$, Bonferroni adjusted). 


\section{Discussion}

We aimed to determine the effects of systematic increases in walking speed on spatiotemporal gait parameters and their variability in people with BVP. We investigated if these parameters would distinguish between healthy participants and participants with BVP, and between patients with BVP who could and could not complete all of the planned walking speed trials (a simple proxy of locomotor capacity). Our hypothesis, that step and double support time and step length variability would systematically reduce with increases in walking speed, whereas step width variability would systematically increase, was confirmed as significant walking speed effects were found for all gait variability parameters. We additionally hypothesized that step and double support time and step length variability at slower walking speeds would be most distinguishing between the healthy control participants and patients with BVP, and also between the patients with BVP that could completely and only partially complete the measurement protocol, whereas step width variability would be most distinguishing between these groups at faster walking speeds. This hypothesis was partly confirmed; step length CV differed between groups BVP and Young and between groups BVP All Gait and BVP Part Gait, double support time CV differed between groups BVP and Young and step width CV differed between groups BVP and Young and BVP and Older for step width variability, but other parameters did not significantly differ at the pairwise comparison level, despite the group effects found for all parameters except step width CV in the BVP All Gait vs. BVP Part Gait analysis.

Regarding our explorative analysis in the patient groups examining correlations between the outcomes of four clinical vestibular tests (vHIT, oVEMP, cVEMP, DVA) and the most distinguishing gait parameters identified, only one significant correlation between the change in logMAR scores during the DVA and the gait parameters were found $(6 \mathrm{~km} / \mathrm{h}$ and Double Support CV; Table 2). One significant correlation of 16 was found between the VEMP thresholds and the gait parameters, but only nine pairs of data were included in this test and if a Bonferroni correction is made for the $\mathrm{p}$ values of these 16 tests, it is no longer significant (oVEMP Left and Step Length CV at $0.4 \mathrm{~m} / \mathrm{s}$; Table 2). Similarly, the one significant correlation between a DVA parameter and gait variability (DVA $6 \mathrm{~km} / \mathrm{h}$ and Double support time CV $0.4 \mathrm{~m} / \mathrm{s}$ ) does not meet the significance threshold if a Bonferroni correction for the 12 tests is made. Even though this study clearly demonstrates the significant contribution of vestibular function to gait, our exploratory analysis confirms the complex contribution of vestibular information during every-day activities and the difficulty in translating current objective clinical measures to highly relevant patient symptoms.

Determining meaningful and distinguishing gait parameters in BVP is vital for the development of interventions, as is using tasks that sufficiently replicate the day-to-day challenges of these patients, to determine candidates for intervention and to assess the effect of those interventions. Two promising interventions currently under development and investigation include noisy galvanic vestibular stimulation (nGVS) and vestibular implants (Guyot et al., 2016, Lewis, 2016, Perez Fornos et al., 2017, Wuehr et al., 2017, Herssens and McCrum, 2019). Discussions of these treatment options can be found elsewhere (Guyot et al., 2016, Wuehr et al., 2017), but it is important to note that both 
show early signs of utility for improving gait in BVP (McCrum et al., 2016, Wuehr et al., 2016). However, it remains to be seen if improvement due to nGVS or a vestibular implant in steady state gait would likewise be seen in more dynamic locomotor task performance, where even unilateral vestibulopathy leads to significantly poorer stability performance (McCrum et al., 2014). It should be noted that while this study examined spatiotemporal variability, differences in dynamic gait stability were not directly assessed and the two are not necessarily equivalent (Dingwell et al., 2001, Bruijn et al., 2013, Perry and Srinivasan, 2017). The parameters presented here represent the amount of variability in the gait parameters, but do not necessarily indicate the overall stability of the participants. Therefore, future work should investigate how dynamic gait stability is altered in BVP and how this is affected by changes in walking speed. Additionally, we suggest that quantification of vestibulospinal reflexes and reflex gains associated with gait stability control in BVP should be conducted, in order to better understand the underlying mechanisms of changes in gait stability in vestibulopathy.

The current study confirmed previous findings of reductions in temporal gait variability and reductions in sagittal plane spatial gait variability in vestibulopathy during faster, compared to slower walking (Schniepp et al., 2012, Wuehr et al., 2016, Schniepp et al., 2017). We extend these previous findings as the current study employed fixed (not self-selected) speeds that were systematically increased, with 120 steps analyzed per speed, thereby improving the reliability of the outcomes. Our findings also align with the growing body of literature indicating a shift from sensory feedback-driven balance control to an increasingly feedforward control with increasing locomotor speed (Brandt et al., 1999, Jahn et al., 2001, Dakin et al., 2013, Forbes et al., 2017, MacNeilage and Glasauer, 2017), but suggest that this may not apply, at least not to the same extent, for mediolateral balance control during gait which may continue to require active control of foot placement in the mediolateral plane. Importantly, the current results further the previous findings by additionally showing that these parameters are related to the locomotor capacities of people with BVP.

We confirmed previously reported increases in step width variability with increasing walking speeds in people with BVP (Wuehr et al., 2016). Previous studies have shown that vestibular perturbations have less impact on direction and variability at higher walking speeds (Fitzpatrick et al., 1999, Jahn et al., 2000) and that the vestibular influence on lower limb muscles is selectively suppressed with increased cadence and speed during walking (Dakin et al., 2013, Forbes et al., 2017). However, the current step width variability results, combined with those of (Wuehr et al., 2016) suggest that vestibular information remains important for mediolateral foot placement at increased walking speeds. During the swing phase when foot placement is coordinated and determined, there is reduced proprioceptive input due to only one foot being in contact with the ground. Therefore, we could reason that vestibular input becomes more important in this phase, and disturbed or lacking vestibular input may decrease foot placement accuracy. These results also provide some explanation as to why people with BVP do not self-select walking speeds that minimize temporal or sagittal plane spatial gait variability (Schniepp et al., 2012, Wuehr et al., 2016, Schniepp et al., 2017). Dramatic increases in step width variability may be undesirable due to reduced stability control or increased 
energetic costs of mediolateral stabilization (Donelan et al., 2004, Dean et al., 2007, O'Connor et al., 2012). Based on the current results, either reason is plausible, as some participants in the BVP Part Gait group did not continue to the faster speeds due to instability, while others could not continue due to being unable to keep up with the speed of the treadmill (implying an energetic or physiological limitation, not a stability-related one). The vestibular influence on gait economy has not yet been thoroughly investigated.

The healthy control groups in this study were not directly age matched with the BVP group, but rather represent healthy participants at the younger and older end of the age range of the BVP group. Variability in step time, double support time and step length of the older group tended to fall between that of the younger and BVP group, showing few statistical differences to either (probably due to a lack of statistical power at the pairwise comparison level). The boxplots seem to indicate that the group Older tend towards the results of group Young for double support time and step length variability. In order to further investigate this issue, we calculated the Cohen's d effect sizes for each group comparison (Young vs. Older, Young vs. BVP and Older vs. BVP) and averaged these across the walking speeds for Step Time CV (0.65, 0.98, 0.39), Step Length CV $(0.95,1.31$, 0.60), Double Support Time CV (0.58, 1.00, 0.50) and Step Width CV (0.21, 1.04, 0.84). These effect sizes confirm that the largest differences were always between the Young and BVP groups, but that the differences between the Older and BVP groups were also always moderate to large, even if not statistically significant, indicating that while age may have been a factor in the Young-BVP comparisons, it certainly does not explain the differences found. However, the group difference in step width variability appear to be more robust, with large significant differences between the BVP group and each healthy group, and no difference due to healthy ageing alone, in agreement with previous studies (Hollman et al., 2011, Herssens et al., 2018). However, other limitations should be kept in mind. Firstly, we did not perform any tests of somatosensory function in the older adult group, and while we think our inclusions criteria "no history of balance or gait difficulties and no history of dizziness" probably deemed somatosensory dysfunction unlikely, it cannot be entirely ruled out. We did however perform the DVA and vHIT tests with nine and eight out of 12 older participants, respectively, which revealed normal function (due to equipment issues, the remaining older adults were not assessed on these tests). Regarding the gait results, we caution comparing the CV of step width to studies of overground walking, as it has been shown in healthy participants that walking on the CAREN results in increased step width variability compared to overground walking (Gates et al., 2012). Additionally, the use of a safety harness may result in small differences to unconstrained overground gait (Decker et al., 2012). Furthermore, treadmill walking appears to be more challenging for people with BVP, evidenced by the fact that the BVP No Gait group were not able to successfully complete the familiarization period, despite reporting being able to walk independently without assistance. We would therefore caution a direct comparison of treadmill-derived gait results with overground gait results in BVP. It should also be noted that the walking speeds used in the current study were not randomized, but progressed from slow to fast, and this could have led to an order effect. We hope that this was minimized by our familiarization protocol, but it cannot be ruled out. This should not have affected our comparisons, however, as all participants followed the same protocol. 
Minor fatigue may have occurred during the assessments, but this should have been minimized as the participants were monitored and breaks were provided when necessary. Regarding the fact that the healthy groups walked with optic flow and the BVP group walked with the virtual environment fixed (so as to provide the same lighting), we do not expect that this difference would have altered our results, as two previous studies found no, or negligible, differences in the parameters assessed here between fixed speed walking with and without virtual reality (Katsavelis et al., 2010, Sloot et al., 2014). The only previous study that did find differences in gait variability due to virtual reality that we are aware of is that of Hollman et al. (2006). However, Hollman et al. (2006) used an insufficient number of data points to reliably assess gait variability (Katsavelis et al., 2010) and used a substantially different virtual reality setup to the current study. Finally, the effect sizes of the difference in step width variability with and without virtual reality and optic flow from Hollman et al. (2006) are much smaller than those found in the current study between Young and BVP All Gait groups at similar walking speeds (Cohen's d of 0.238-0.657 in Hollman et al. (2006) vs. 1.064-1.382 in the current study).

We also acknowledge that our division of participants into the BVP All Gait and BVP Part Gait groups is based on a rather simple criterion. Of the 12 participants in the BVP Part Gait group, the range of locomotor capacities within this group is likely broad. Reasons for lack of completion also varied across the participants, with some stopping due to lack of stability control (too much lateral deviation with a risk of stepping off the treadmill) and others unable to keep up with a faster belt speed. Nevertheless, we found significant group effects on gait variability, indicating the potential association between gait variability and overall locomotor capacity in BVP. Further research into gait parameters that can distinguish between patients with different functional limitations is encouraged to aid the development of accurate diagnostic functional testing protocols.

In conclusion, spatiotemporal gait parameters and their variability show speeddependency in people with BVP and in healthy adults. In particular, step length variability at slower speeds and step width variability at faster speeds were the most distinguishing parameters between the healthy participants and people with BVP, and within groups with BVP who have different locomotor capacities. Gait variability in BVP was generally not correlated with the clinical tests of vestibular function. The current findings indicate that analysis of gait variability at multiple speeds has potential as an assessment tool for vestibular interventions.

\section{Acknowledgements}

The authors thank Wouter Bijnens, Rachel Senden and Rik Marcellis for their support with the measurements. FL was financially supported by MED-EL. 


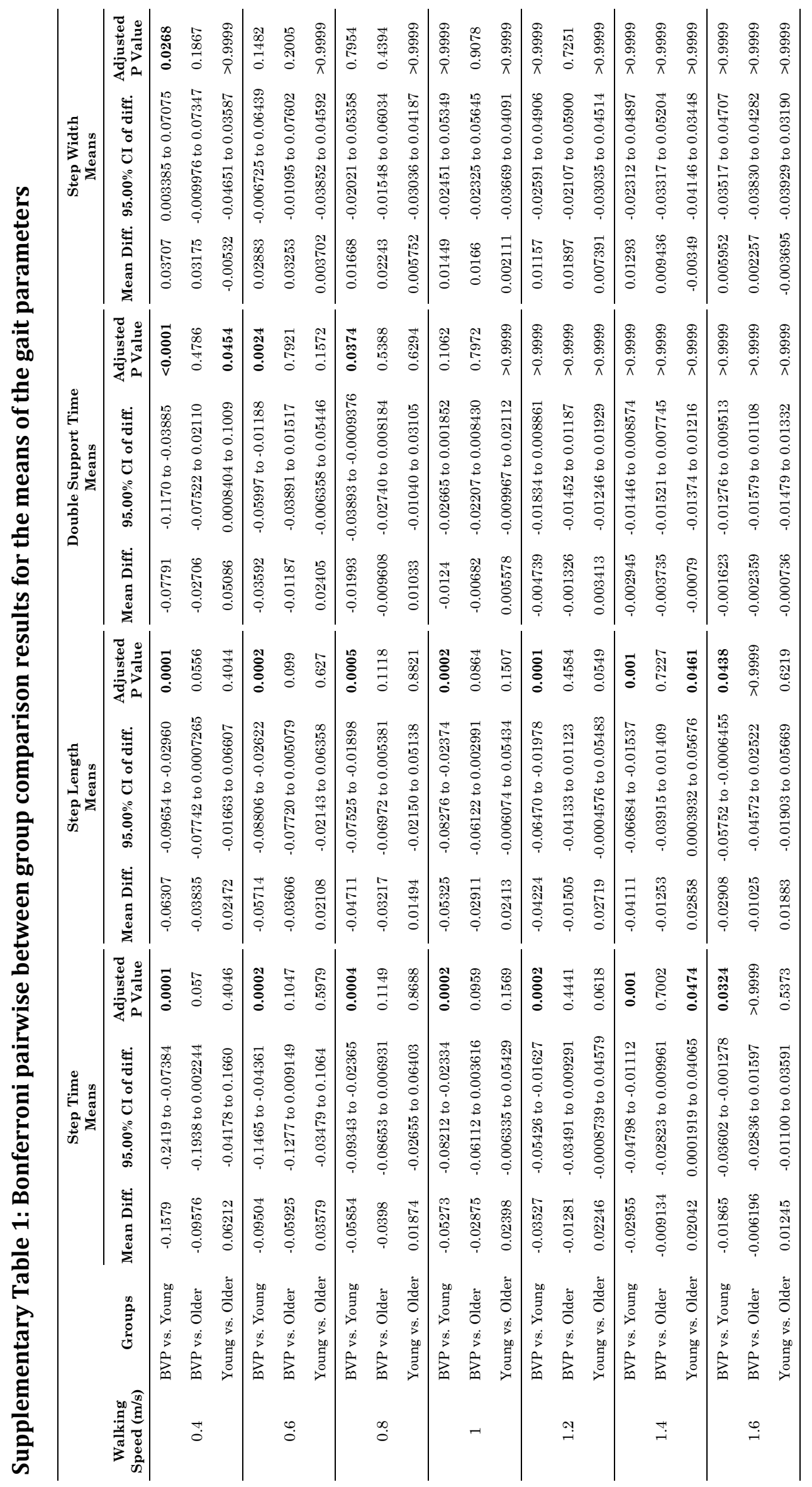




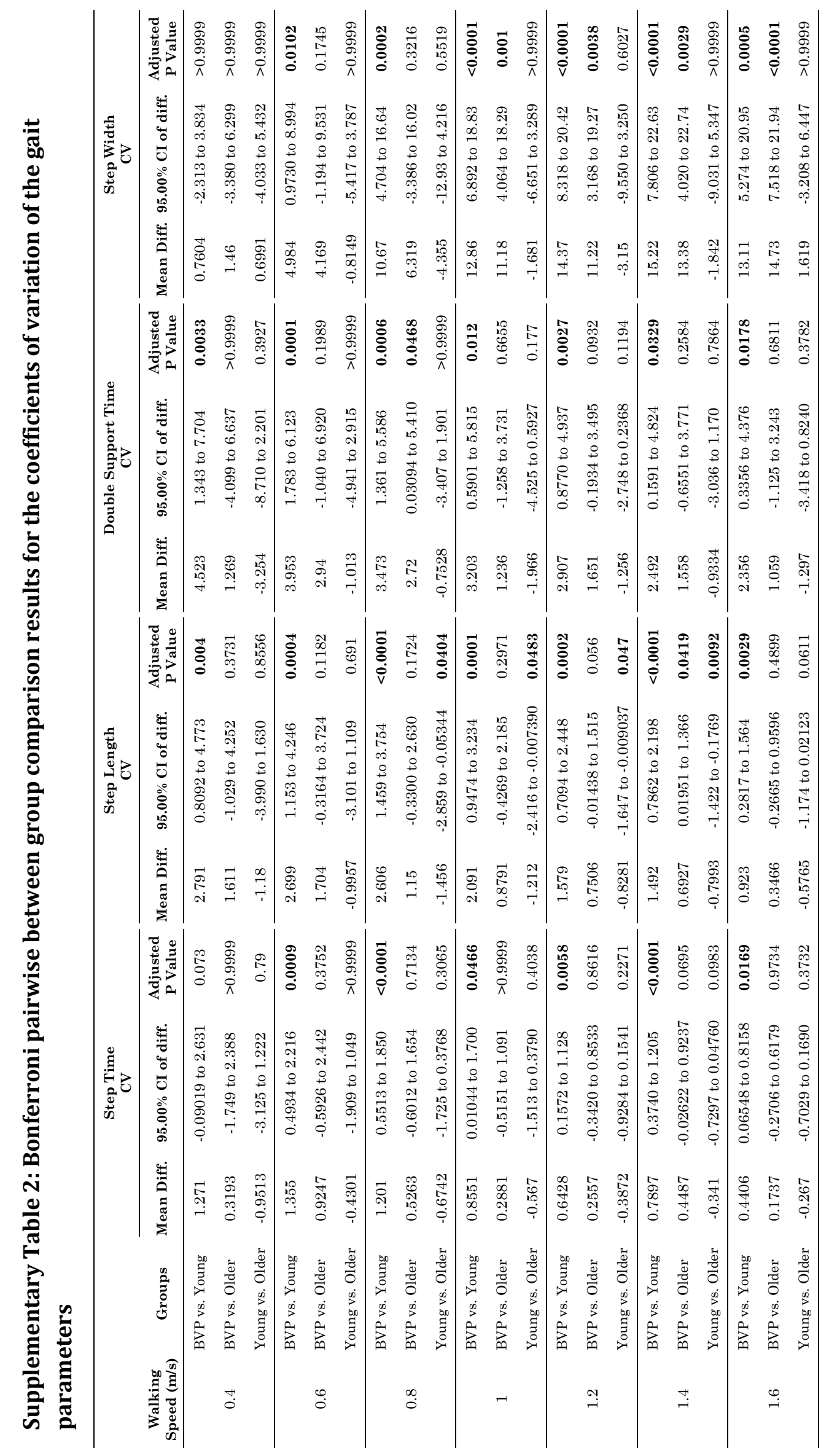




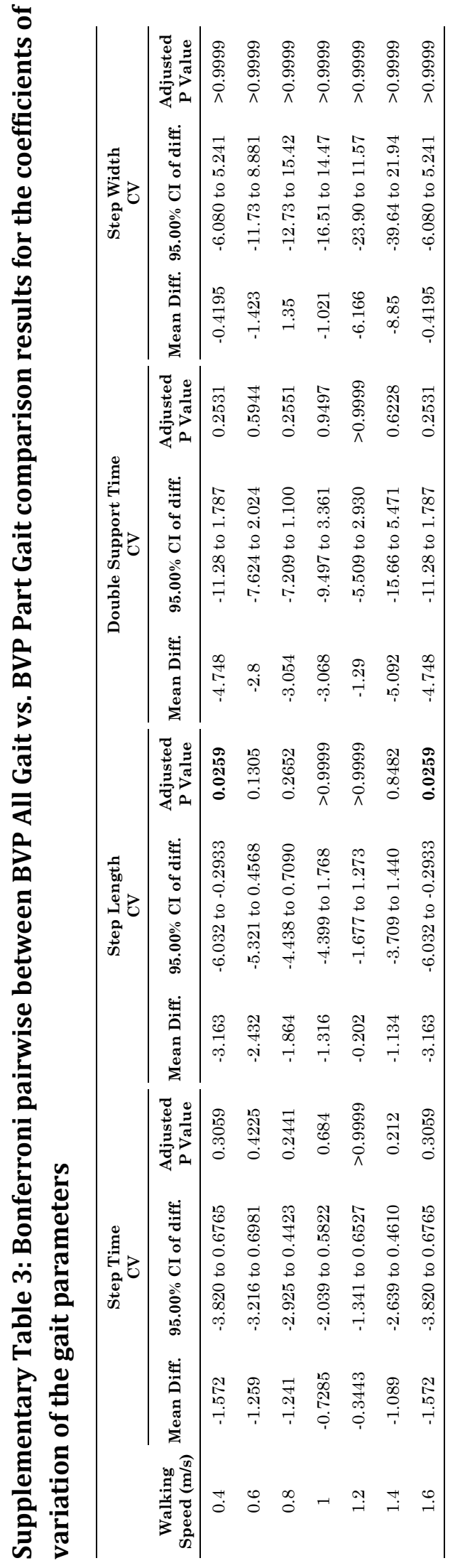


2.1: The walking speed-dependency of gait variability in bilateral vestibulopathy and its association with clinical tests of vestibular function 


\section{2:}

\section{Locomotor stability and adaptation during perturbed walking across the adult female lifespan}

Christopher McCrum, Gaspar Epro, Kenneth Meijer, Wiebren Zijlstra,

Gert-Peter Brüggemann, Kiros Karamanidis

\section{Published as:}

MCCRUM, C., EPRO, G., MEIJER, K., ZIJLSTRA, W., BRÜGGEMANN, G. P. \& KARAMANIDIS, K. 2016. Locomotor stability and adaptation during perturbed walking across the adult female lifespan. J Biomech, 49, 1244-7. doi: 10.1016/j.jbiomech.2016.02.051

(C) 2016. This manuscript version is made available under the CC-BY-NC-ND 4.0 license.

http://creativecommons.org/licenses/by-nc-nd/4.0/ 


\begin{abstract}
The aim of this work was to examine locomotor stability and adaptation across the adult female lifespan during perturbed walking on the treadmill. 11 young, 11 middle and 14 older-aged female adults (mean and SD: 25.5(2.1), 50.6(6.4) and 69.0(4.7) years old respectively) walked on a treadmill. We applied a sustained perturbation to the swing phase of the right leg for 18 consecutive gait cycles, followed by a step with the resistance unexpectedly removed, via an ankle strap connected to a break-and-release system. The margin of stability (MoS) at foot touchdown was calculated as the difference between the anterior boundary of the base of support (BoS) and extrapolated center of mass. Older participants showed lower MoS adaptation magnitude in the early adaptation phase (steps 1-3) compared to the young and middle-aged groups. However, in the late adaptation phase (steps 16-18) there were no significant differences in adaptation magnitude between the three age groups. After removing the resistance, all three age groups showed similar aftereffects (i.e. increased BoS). The current results suggest that in old age, the ability to recalibrate locomotion to control stability is preserved, but the rate of adaptive improvement in locomotor stability is diminished.
\end{abstract}




\section{Introduction}

Reducing falls in the elderly is vital for public health, due to consequences such as fractures, functional decline and death (Terroso et al., 2013). Older adults fall most often during ambulation, frequently due to tripping or slipping (Talbot et al., 2005). Reactive and predictive adjustments of gait contribute to locomotor stability (state of the center of mass $(\mathrm{CoM})$ in relation to the base of support $(\mathrm{BoS})$ ) and decrease the risk of falling when facing a challenging walking environment (Bierbaum et al., 2010, Bierbaum et al., 2011). One such adjustment is rapid stepping, which is diminished in old age (Maki and McIlroy, 2006). Step length and time following release from a forward lean indeed predict older adults' maximum recoverable forward lean angle (Graham et al., 2015), which predicts future falls (Carty et al., 2015).

Improving compensatory stepping adjustments may help in preventing falls (Grabiner et al., 2014). In older adults, experiencing laboratory-induced gait perturbations has led to improved recovery responses to various mechanical perturbations (Bierbaum et al., 2010, Pai et al., 2010, Bierbaum et al., 2011, Bhatt et al., 2012, Pai et al., 2014b). More importantly, perturbation training may reduce falls by up to $50 \%$ in the following year (Rosenblatt et al., 2013, Pai et al., 2014a). However, it remains partly unclear how locomotion adapts to control stability in response to perturbations, and how this ability declines across the adult lifespan. Determining how locomotor stability and adaptation are affected across the female lifespan is of particular importance, as locomotor stability declines, and falls increase in middle age in women (Talbot et al., 2005, Süptitz et al., 2013). Women over 45 have an increased susceptibility to bone fractures (Donaldson et al., 1990), and experience more fall-related injuries (Talbot et al., 2005) and hip fractures (Parkkari et al., 1999) than men.

Bruijn et al. (2012) found that older adults show diminished step length adaptation in response to a split-belt walking paradigm (one belt at $1 \mathrm{~m} / \mathrm{s}$, the other at $0.5 \mathrm{~m} / \mathrm{s}$ ) compared with younger adults. Bierbaum et al. (2010), however, found that older adults are capable of adapting their dynamic stability to a similar or greater magnitude as young adults to surface change perturbations. Additionally, Pai et al. (2010) showed that older adults are capable of predictive and reactive adaptations to slip perturbations. In general however, there is a lack of studies on aging and locomotor stability and adaptation and importantly, no study has analyzed how the adaptability of locomotion to perturbations is affected across the adult lifespan using a cross sectional design. This study aimed to examine locomotor stability and adaptation in young, middle and older-aged adults in response to a sustained resistance perturbation during walking, in order to test the hypothesis that older adults remain capable of adapting their locomotion to external gait perturbations in order to maintain stability, but not to the same extent as young and middle-aged adults.

\section{Methods}

Eleven young (mean and SD: 25.5(2.1) years), 11 middle-aged (50.6(6.4) years) and 14 older (69.0(4.7) years) healthy women, with no known musculoskeletal or neurological 
deficits, participated in this study. The height and body mass for each group were $166.9(4.3) \mathrm{cm}$ and $62.7(6.8) \mathrm{kg}$ for the young, $168.2(4.8) \mathrm{cm}$ and $66.4(7.9) \mathrm{kg}$ for the middleaged, and $161.2(5.0) \mathrm{cm}$ and $67.3(4.7) \mathrm{kg}$ for the older adults respectively. Participants walked at $1.4 \mathrm{~m} / \mathrm{s}$ on a treadmill (pulsar $4.0, \mathrm{~h} / \mathrm{p} /$ cosmos; Nussdorf-Traunstein, Germany) to standardize the walking speed and perturbation. One treadmill walking familiarization session took place four to seven days before measurements. Participants wore a safety harness connected to an overhead frame during the measurements. The procedures were explained to the participants prior to obtaining informed consent in accordance with the Declaration of Helsinki. The study was approved by the German Sport University Cologne ethical board.

The gait perturbation was applied using a custom built brake-and-release system described previously (Süptitz et al., 2013, McCrum et al., 2014) which applies and removes $2.1 \mathrm{~kg}$ of resistance to the leg via an ankle strap and Teflon cable, controlled via an electronically driven magnet system. Resistance is turned on during the right leg stance phase and removed following right foot touchdown, so that the entire swing phase is affected. The measurement periods included familiarization, baseline, single perturbation, washout, sustained perturbation and post-perturbation. Familiarization consisted of ten minutes walking at $1.4 \mathrm{~m} / \mathrm{s}$. The ankle strap was then attached to the right leg and participants walked for a further four minutes. A baseline measurement (non-perturbed walking; approximately 20 seconds) was recorded at the end of this period. Six consecutive steps were used to determine a baseline for the assessed parameters. The resistance was then applied for one step and immediately removed. Following a two to three minute washout period of non-perturbed walking, the resistance was again applied, this time left on for 18 consecutive steps of the right leg, followed by a final step of the right leg with the resistance removed (post-perturbation), in order to examine aftereffects. Aftereffects were analyzed using the BoS, as recalibrations were expected to be most noticeable in this variable. Participants were not warned about the onset or removal of the perturbation, but were informed that a resistance would be applied at some point during walking.

Our method to assess gait stability on the treadmill has been described in previous studies (Süptitz et al., 2012, Süptitz et al., 2013, McCrum et al., 2014), and a detailed description is provided in the appendix (see Supplementary Material). Briefly, a full kinematic model was tracked using 26 markers and an eight camera (120 Hz) Vicon Nexus motion capture system (Vicon Motion Systems, Oxford, UK). The anteroposterior margins of stability (MoS) were calculated at foot touchdown (determined using tibia accelerometer data (ADXL250; Analog Devices, Norwood, MA, USA)) as the difference between the anterior boundary of the BoS (anteroposterior component of the toe projection to the ground) and the extrapolated CoM as defined by Hof et al. (2005).

The parameters of interest were MoS at baseline, at touchdown of the perturbed step during the single perturbation period and of the first six and last five steps during the sustained perturbation period. This method was used due to recording limitations of the motion capture system, which could not record all 18 stride cycles. In order to examine locomotor adaptation, we calculated the MoS adaptation magnitude in a similar manner to Bierbaum et al. (2011) for the early and late adaptation phases of the sustained perturbation period as follows: 


$$
\text { Adaptation Magnitude }=\left(1-\frac{M o S_{\text {AdaptPeriod }}-M o S_{\text {Base }}}{M o S_{\text {Single }}-M o S_{\text {Base }}}\right) \times 100
$$

where $M o S_{\text {AdaptPeriod }}$ is either the mean MoS of the first or last three perturbed steps (early or late adaptation period), $M o S_{\text {Single }}$ is the MoS at touchdown of the perturbed step during the single perturbation period, and $M o S_{\text {Base }}$ is baseline MoS. Finally, in order to examine aftereffects, we compared the BoS during baseline with the step after the unexpected removal of the resistance.

A two-way repeated measures ANOVA with age and step (baseline, single perturbation period step, and the first six and last five steps of the sustained perturbation period) as factors was used to examine age and step related differences in the MoS. Differences in adaptation magnitude were examined using a two-way repeated measures ANOVA with age and adaptation phase (early and late) as factors. To assess potential aftereffects in the BoS, a two-way repeated measures ANOVA with age group and task period (base and post-perturbation) as factors was conducted. Duncan's Tests were applied for pairwise comparisons. The significance level was $\alpha=0.05$. Results are presented as mean and standard error.

\section{Results}

A significant age by step interaction was found for the MoS $(p<0.05)$ indicating that the effect of age on the MoS was step specific (Fig. 1). Post-hoc tests revealed no significant differences between groups during baseline. During the single perturbation period, all groups demonstrated significantly lower MoS in comparison to baseline $(p<0.05)$ with no between age group differences. Significantly lower MoS compared to baseline was found for all groups for all steps in the sustained perturbation period $(p<0.05)$. The older age group demonstrated significantly lower MoS for the first six steps of the sustained perturbation period $(p<0.05)$ compared with the younger groups, however there were no significant differences in MoS between the age groups for the last five steps (steps 14-18; $p>0.05)$.

Regarding the adaptation magnitude, the ANOVA revealed a significant age by adaptation phase interaction ( $p<0.05$; Fig. 2). Post-hoc tests revealed significant differences between age groups only during the early adaptation phase, with the older group showing a significantly lower adaptation magnitude $(p<0.05$; Fig. 2$)$. At touchdown of the final measured step with the resistance unexpectedly removed, significant aftereffects were seen in all groups, with a significantly increased $\operatorname{BoS}(p<0.05$; Fig. 3$)$ compared to baseline, with no significant differences found between the age groups $(p>$ 0.05; Fig. 3). 


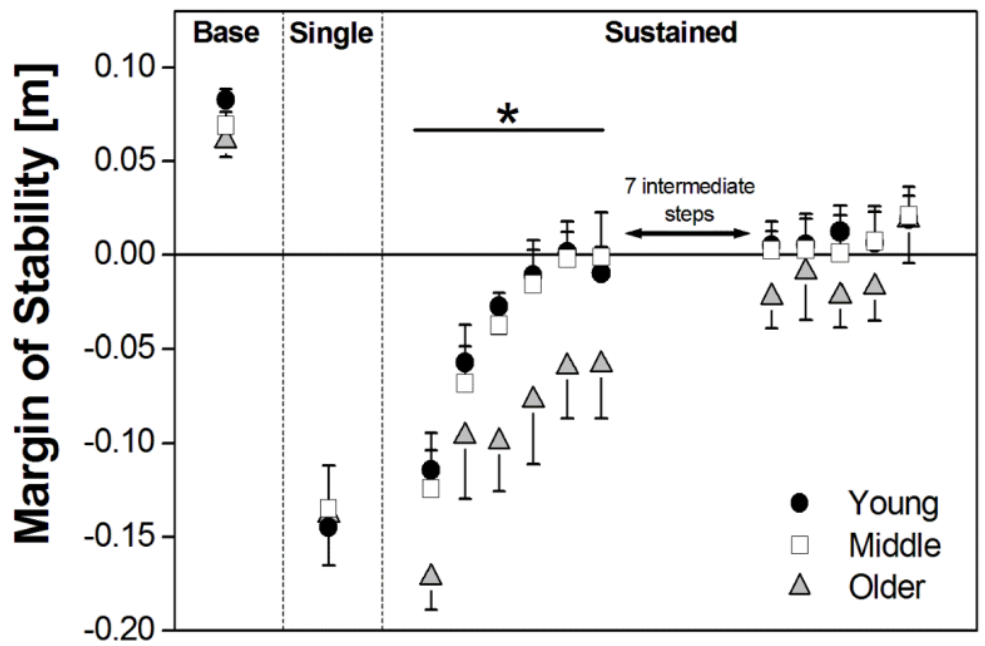

Fig. 1: Margin of stability (MoS) analysis. MoS at foot touchdown (mean and SE) during nonperturbed walking (Base: average of 6 consecutive steps) and during the single and sustained perturbation periods for 11 young, 11 middle-aged and 14 older female adults while walking on the treadmill at $1.4 \mathrm{~m} / \mathrm{s}$. All single and sustained perturbation period values were significantly lower than baseline for all groups $(p<0.05)$. : Statistically significant difference between the older group and the young and middle-aged groups with no differences between the young and middle-aged groups $(p<0.05)$.

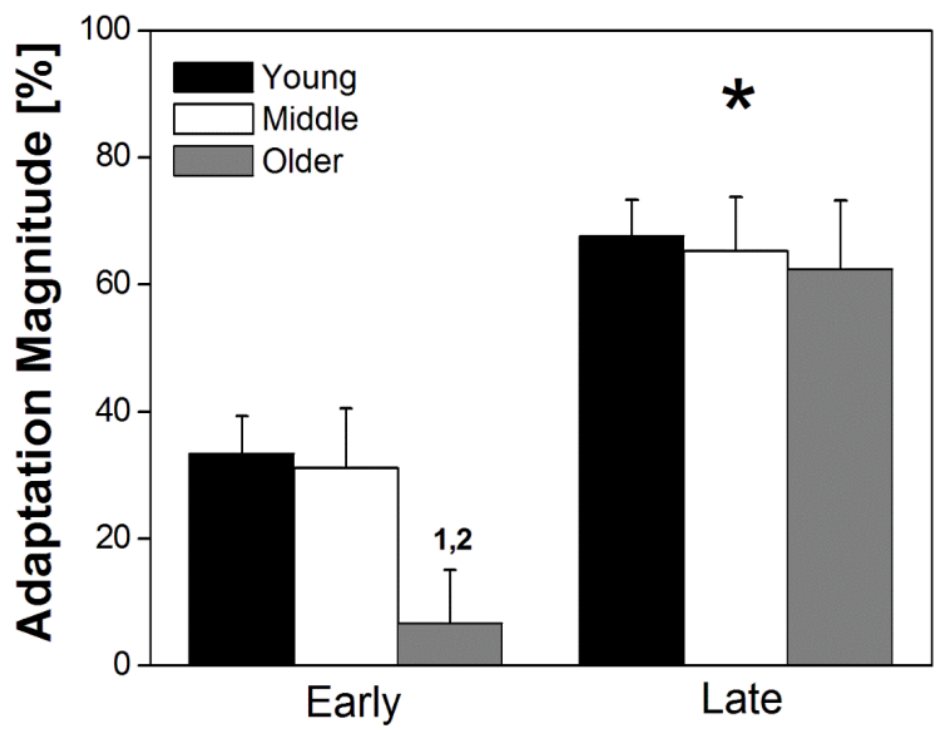

Fig. 2: Adaptation magnitude in MoS in the early and late adaptation phases. The adaptation magnitude for all groups in the early (first three steps) and late (last three steps) adaptation phases in MoS in reference to the single perturbation period. 1 and 2: Statistically significant difference during the early adaptation period to the young and middle-aged groups respectively $(p<0.05)$. *: Statistically significant difference to the early adaptation period for all groups $(p<0.05)$. 


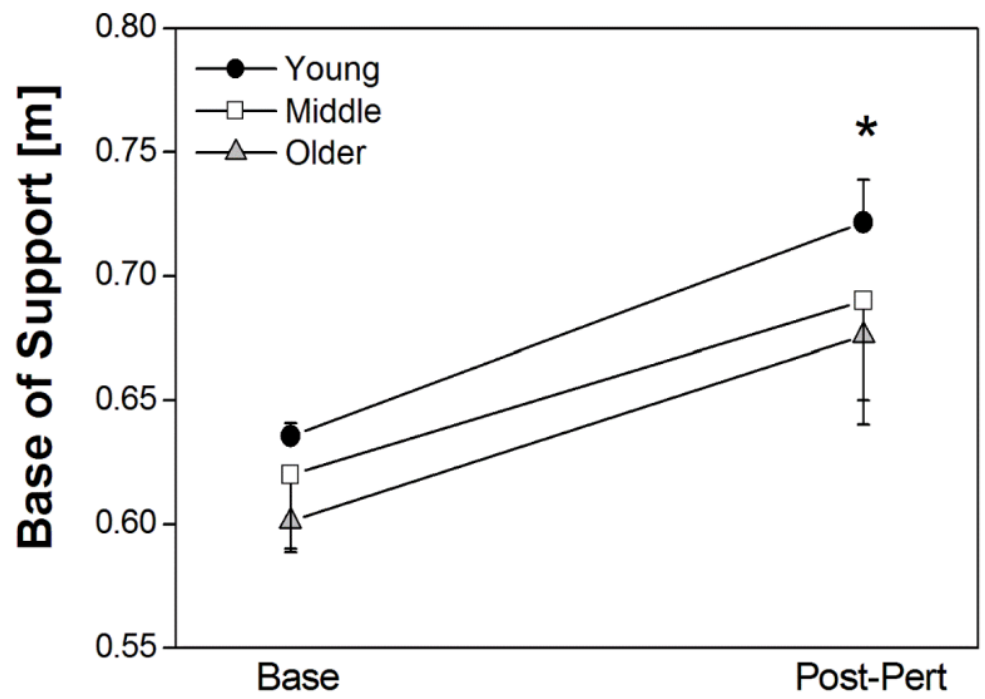

Fig. 3: Aftereffects in the BoS. The BoS for all age groups during non-perturbed walking (Base) and in the first step after the resistance was unexpectedly removed (Post-Pert). *: Statistically significant difference in the BoS between Post-Pert and Base for all groups $(p<$ 0.05).

\section{Discussion}

We aimed to examine locomotor stability and adaptation in response to a sustained perturbation in young, middle and older-aged female adults. Our hypothesis, that older adults remain capable of adapting to external gait perturbations, but not to the same extent as younger adults, was partly supported, as the older adults demonstrated a similar adaptation magnitude of the MoS to the young and middle-aged adults by the end of the sustained perturbation period. However, in the first three steps of the sustained perturbation period, the older adults showed significantly lower MoS adaptation magnitude compared to the young and middle-aged groups, suggesting that while they could adapt to a similar magnitude, this occurred slower than in the younger groups. This declined rate of adaptation was not present in middle-aged adults.

The MoS adaptation magnitude was significantly decreased in the early adaptation phase for the older group compared to the other groups. This indicates that adaptations made following the initial single perturbation were not as substantial in the older group as in the other groups, indicating a higher risk for older adults in situations with perturbations in quick succession (e.g. uneven terrain). That being said, all age groups demonstrated significant improvements in MoS adaptation magnitude by the late adaptation phase, suggesting that the locomotor adaptability was not diminished in any age group. Similar results were seen in the BoS, showing that older adults struggled to cope with the early perturbations (see Fig. S1 in the Supplementary Material).

Our analysis of the aftereffects in the BoS support the role of a recalibration of locomotion in our participants, as all subject groups showed a significant increase in the 
BoS after the resistance was removed (Fig. 3). These findings suggest that the recalibration of motor commands may not be significantly affected by aging, although adaptation may occur more quickly in younger than in older adults. The aftereffects indicate either, that older adults are able to retain adaptations after only a few perturbations, or that they demonstrate perseverance after resistance removal. However, to specifically investigate this, many more perturbations would be required, which due to the task's physical demand, was not considered feasible.

While Bruijn et al. (2012) reported deficient step length adaptation in older adults, their walking speed was substantially slower than our $1.4 \mathrm{~m} / \mathrm{s}$, which may not have required such significant adaptation rates or magnitudes to continue safe forward progression of gait. An additional consideration is the range of different perturbations used across studies, as these could have different effects on the magnitude of adaptations required to maintain stability. A final consideration is that we analyzed three age cohorts, and did not longitudinally follow participants as they grew older, and therefore, our conclusions should be interpreted with this in mind.

In conclusion, our results provide evidence that with aging, the ability to recalibrate locomotion to control stability is preserved. However, a deficient rate of locomotor adaptation can be seen in old age, which may have implications for training interventions and falls prevention.

\section{Acknowledgements}

We thank Thomas Förster and Jürgern Geiermann and their teams for technical assistance. Funding was provided by the Forschungsservicestelle, German Sport University Cologne. 


\section{Supplementary Material}

\section{Methods}

\section{Margin of Stability Analysis}

Our twelve segment full body kinematic model consisted of left and right feet, left and right lower legs, left and right thighs, trunk, left and right lower arms, left and right upper arms, and head. 26 retro reflective markers (radius $16 \mathrm{~mm}$ ) were attached to anatomical landmarks on the skin and the 3D coordinates of the markers were recorded by the Vicon Nexus motion capture system. The motion capture system was comprised of eight infrared cameras operating at $120 \mathrm{~Hz}$ and the $3 \mathrm{D}$ coordinates of the markers were smoothed using a Woltring filter routine with a mean squared error of five (Woltring, 1986). Segmental masses and their location were calculated based on the data reported by Dempster et al. (1959). Foot touchdown was determined using tibial acceleration data, measured by twodimensional accelerometers (ADXL250; Analog Devices, Norwood, MA, USA) and in addition, foot touchdowns were visually checked by the investigators using a video camera $(60 \mathrm{~Hz})$ which was synchronised with the motion capture and accelerometry data. The anteroposterior margins of stability (MoS) during gait were calculated as the difference between the anterior boundary of the base of support (BoS; anteroposterior component of the projection of the toe to the ground) and the extrapolated center of mass ( $\mathrm{X}_{\mathrm{CoM}}$ ) as defined by Hof et al. (2005). This method has been previously validated by Arampatzis et al. (2008). We also considered the anteroposterior velocity of the BoS (the average velocity of the toe marker during the stance phase, which is approximately equal to the treadmill belt speed) as in previous work (Süptitz et al., 2012, Süptitz et al., 2013, McCrum et al., 2014). $\mathrm{X}_{\mathrm{CoM}}$ was defined as follows:

$$
\mathrm{X}_{\mathrm{CoM}}=\mathrm{P}_{\mathrm{CoM}}+\frac{\left(\mathrm{V}_{\mathrm{CoM}}+\left|\mathrm{V}_{\mathrm{BoS}}\right|\right)}{\sqrt{\frac{\mathrm{g}}{\mathrm{L}}}}
$$

where $\mathrm{P}_{\mathrm{CoM}}$ represents the anteroposterior component of the projection of the CoM to the ground, $\mathrm{V}_{\mathrm{CoM}}$ represents the anteroposterior velocity of the CoM, $\mathrm{V}_{\mathrm{Bos}}$ represents the anteroposterior velocity of the anterior boundary of the BoS, $\mathrm{g}$ is acceleration due to gravity and $\mathrm{L}$ is the distance between the CoM and the center of the ankle joint in the sagittal plane. The BoS was defined as the distance between the anterior boundaries of the leading and trailing foot at touchdown, using the distance between the vertical projections of the respective toe markers. All anteroposterior positions determined from the motion capture data (CoM, $\mathrm{X}_{\mathrm{CoM}}$, and $\mathrm{BoS}$ anterior boundary) were normalized to the toe marker of the trailing foot (BoS posterior boundary), in order to account for differences in participants' locations on the treadmill. 


\section{Results}

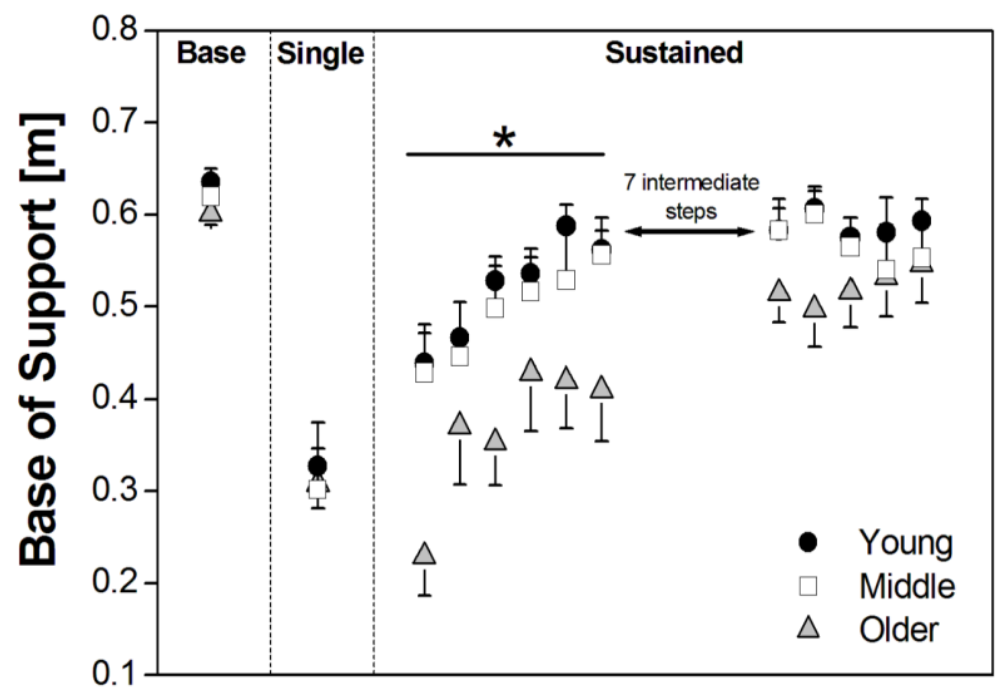

Fig. S1: Base of Support (BoS) analysis. BoS at foot touchdown (mean and SE) during nonperturbed walking (Base: average of 6 consecutive steps) and during the single and sustained perturbation periods for 11 young, 11 middle-aged and 14 older female adults while walking on the treadmill at $1.4 \mathrm{~m} / \mathrm{s}$. A two-way repeated measures ANOVA with age and step (baseline, single perturbation period step, and the first six and last five steps of the sustained perturbation period) as factors revealed a significant age by step interaction. Post-hoc tests revealed no significant differences between groups during baseline. During the single perturbation period, all groups demonstrated significantly lower BoS in comparison to baseline $(p<0.05)$ with no between age group differences. The older age group demonstrated significantly lower BoS for the first six steps of the sustained perturbation period $(p<0.05)$ compared with the younger groups. *: Statistically significant difference between the older group and the young and middle-aged groups with no differences between the young and middle-aged groups $(p<0.05)$. 
2.2: Locomotor stability and adaptation during perturbed walking across the adult female lifespan 


\section{Part Three: \\ Gait Perturbation Methodology}

"There are so many ways to account for negative outcomes that it is safer to doubt one's methods before doubting one's subjects."

- Frans de Waal

"Perfection is not attainable. But if we chase perfection, we can catch excellence."

- Vince Lombardi 


\section{1:}

\section{A systematic review of gait perturbation paradigms for improving reactive stepping responses and falls risk among healthy older adults}

Christopher McCrum, Marissa H. G. Gerards, Kiros Karamanidis, Wiebren Zijlstra, Kenneth Meijer

\section{Published as:}

MCCRUM, C., GERARDS, M. H. G., KARAMANIDIS, K., ZIJLSTRA, W. \& MEIJER, K. 2017. A systematic review of gait perturbation paradigms for improving reactive stepping responses and falls risk among healthy older adults. Eur Rev Aging Phys Act, 14, 3. doi: 10.1186/s11556-017-0173-7

(C) The Authors 2017. This work is licensed under the Creative Commons Attribution 4.0 International License. http://creativecommons.org/licenses/by/4.0/ 


\begin{abstract}
Background: Falls are a leading cause of injury among older adults and most often occur during walking. While strength and balance training moderately improve falls risk, training reactive recovery responses following sudden perturbations during walking may be more task-specific for falls prevention. The aim of this review was to determine the variety, characteristics and effectiveness of gait perturbation paradigms that have been used for improving reactive recovery responses during walking and reducing falls among healthy older adults.
\end{abstract}

Methods: A systematic search was conducted in PubMed, Web of Science, MEDLINE and CINAHL databases in December 2015, repeated in May 2016, using sets of terms relating to gait, perturbations, adaptation and training, and ageing. Inclusion criteria: studies were conducted with healthy participants of 60 years or older; repeated, unpredictable, mechanical perturbations were applied during walking; and reactive recovery responses to gait perturbations or the incidence of laboratory or daily life falls were recorded. Results were narratively synthesised. The risk of bias for each study (PEDro Scale) and the levels of evidence for each perturbation type were determined.

Results: In the nine studies that met the inclusion criteria, moveable floor platforms, ground surface compliance changes, or treadmill belt accelerations or decelerations were used to perturb the gait of older adults. Eight studies used a single session of perturbations, with two studies using multiple sessions. Eight of the studies reported improvement in the reactive recovery response to the perturbations. Four studies reported a reduction in the percentage of laboratory falls from the pre- to post-perturbation experience measurement and two studies reported a reduction in daily life falls. As well as the range of perturbation types, the magnitude and frequency of the perturbations varied between the studies.

Conclusions: To date, a range of perturbation paradigms have been used successfully to perturb older adults' gait and stimulate reactive response adaptations. Variation also exists in the number and magnitudes of applied perturbations. Future research should examine the effects of perturbation type, magnitude and number on the extent and retention of the reactive recovery response adaptations, as well as on falls, over longer time periods among older adults. 


\section{Background}

Falls are a leading cause of injury among older adults, with hip fractures (Lord et al., 1993, Cali and Kiel, 1995, Terroso et al., 2013, Boye et al., 2014) and head injuries (Terroso et al., 2013, Boye et al., 2014) among the more severe consequences. Falls most often occur during walking (Tinetti et al., 1988, Berg et al., 1997, Li et al., 2006, Kelsey et al., 2012, Robinovitch et al., 2013), which is also the most common activity prior to falls that lead to injury or hospital admission (Cali and Kiel, 1995, Boye et al., 2014). Slipping and tripping during walking are the most common causes of falls among older adults (Blake et al., 1988, Tinetti et al., 1988, Downton and Andrews, 1991, Lord et al., 1993, Berg et al., 1997, Li et al., 2006), which represent failures to predictively (before the perturbation) or reactively (after the perturbation) adapt to changes and challenges in the environment. Therefore, there is a need to physically prepare older adults for situations where unexpected mechanical disturbances to gait could occur.

Lower limb muscle strength (Grabiner et al., 2005, Karamanidis et al., 2008, Pijnappels et al., 2008a, Pijnappels et al., 2008b, Carty et al., 2012a, Carty et al., 2012b, Senden et al., 2014, Graham et al., 2015, Ding and Yang, 2016, Epro et al., 2016) and tendon stiffness (Karamanidis et al., 2008) have been associated with stability recovery performance following different balance and gait perturbations, with greater muscle strength and tendon stiffness generally showing modest associations with more effective stability maintenance or recovery. General exercise interventions (combinations of strength, balance and aerobic exercises) have generally resulted in moderate reductions (14-17\%) in falls incidence among older adults (Chang et al., 2004, Sherrington et al., 2008, Gillespie et al., 2012), which reflects the modest associations observed between muscletendon properties and stability performance. While these reductions are statistically significant, the fact that greater reductions in falls incidence are not seen may be explained by the fact that general exercise interventions often lack specificity to the balance recovery mechanisms that are needed following balance loss, such as compensatory stepping, counter rotation or grasping actions (Maki and McIlroy, 2006, Hof, 2007). Balance maintenance requires a complex interaction of several mechanisms and hence, improved balance control in one task in particular is not likely to be of benefit during other tasks (Kummel et al., 2016). Accordingly, only negligible associations between static posturography and dynamic stability performance (forward lean-andrelease and slip/trip recovery tasks) have been reported (Owings et al., 2000, Mackey and Robinovitch, 2005, Bhatt et al., 2011, McCrum et al., 2017a). Therefore, testing and training tasks more specific to balance recovery mechanisms may provide more insight and benefit for falls reduction and prevention.

It has previously been suggested that training involuntary compensatory recovery responses following sudden perturbations is more task specific than general strength and balance exercise for preventing a fall after a loss of balance (Oddsson et al., 2007, Grabiner et al., 2008, Granacher et al., 2011, Grabiner et al., 2014). Even voluntary stepping exercise, such as multidirectional stepping to targets, is not as specific as involuntary, reactive compensatory stepping where faster movement speeds and an inability to make use of anticipatory postural adjustments are characteristic (Luchies et al., 1999, Oddsson et al., 2007, Granacher et al., 2011). Eliciting involuntary, reactive compensatory stepping 
by applying unexpected perturbations during walking also increases the task specificity, as most falls occur during walking (Tinetti et al., 1988, Berg et al., 1997, Li et al., 2006, Kelsey et al., 2012, Robinovitch et al., 2013). However, in order to benefit from this kind of training, the participants must be capable of adapting their reactive recovery responses during gait.

Reactive recovery responses are required to cope with unexpected perturbations to gait in order to continue safe locomotion. We define reactive recovery responses here as feedback-driven adaptations in gait in response to mechanical disturbances to the regular gait pattern. The first step of such a response is recognising the onset of the perturbation, achieved though integration of visual, somatosensory and vestibular sensory information. The contribution of each sensory system may vary with perturbation type due to differences in the perception of motion (Arena et al., 2016). Stability can then be recovered through a number of strategies, such as compensatory stepping, counter rotation or grasping actions (Hof et al., 2005, Maki and McIlroy, 2006). In situations where compensatory stepping is required to maintain balance, the spatiotemporal characteristics of the step (e.g. direction, timing and amplitude) need to match the requirements for optimal control of stability given the specific environmental constraints. Such reactive responses appear to involve spinal locomotor networks, as chronic spinal cats (Forssberg et al., 1975, Forssberg et al., 1977), as well as human infants prior to independent walking (Lam et al., 2003) exhibit well organised reflex responses (increased swing limb flexion and limb flexor activation) to paw and foot touches during leg swing that simulate a potential trip hazard. Critically, adaptation of these responses has been reported following repeated paw and foot (dorsum) touches in spinal cats (Zhong et al., 2012) and human infants (Pang et al., 2003), suggesting that spinal locomotor networks are plastic. Therefore, older adults' reactive recovery responses following gait perturbations may, in part, be improved with repetition via these reflexes. While there is evidence to suggest that certain neurological patient populations may be limited in their reactive adaptation potential during gait (patients with vestibulopathy (McCrum et al., 2014) and Parkinson's disease (Moreno Catala et al., 2016), for example), the ability to adapt in a reactive or predictive manner to repeated perturbations appears to be largely unaffected by nonpathological ageing (Pavol et al., 2004, Barrett et al., 2012, Carty et al., 2012c, McCrum et al., 2014, Bohm et al., 2015, Dijkstra et al., 2015, McCrum et al., 2016a).

When applied in prevention and rehabilitation settings, the use of sudden, unexpected mechanical perturbations during stance or gait is often termed perturbationbased balance training (Mansfield et al., 2010, Bieryla and Madigan, 2011, Granacher et al., 2011, Mansfield et al., 2015b). The goal of such training is to target the specific mechanisms of balance recovery related to reducing falls such as compensatory stepping, counter rotation or grasping actions (Hof et al., 2005, Maki and McIlroy, 2006). Aside from these movement strategies to maintain balance, factors such as reaction time, perception of losses of balance and speed of sensory information processing are challenged and may improve with perturbation training. Two recent meta-analyses of randomised controlled trials (RCTs) have reported significantly lower post-training falls incidence among older adults who took part in such training (note that Okubo et al. (2017) also included voluntary stepping training interventions in their meta-analysis) (Mansfield et al., 2015b, Okubo et 
al., 2017). These meta-analyses focussed on RCTs that assessed falls incidence, which may mean that non-RCT studies or studies that did not report falls data, but nonetheless included relevant information on reactive recovery responses following perturbations, may have been omitted. Additionally, of the included studies in these meta-analyses, only four were conducted with healthy older adults and applied sudden, unexpected perturbations during walking (Bhatt et al., 2012, Parijat and Lockhart, 2012, Lurie et al., 2013, Pai et al., 2014a), three of which used very similar perturbation paradigms. As a result, it is difficult to determine, based on these studies, the variety, characteristics (e.g. perturbation type, magnitude, standardisation, scaling, progression etc.) and effectiveness of gait perturbation paradigms that could be used with older adults for improving reactive recovery responses and preventing or reducing falls. Therefore, we systematically searched for all studies that applied unexpected mechanical disturbances during walking in healthy older adults and assessed changes in reactive recovery responses or falls incidence, in order to determine the variety, characteristics and effectiveness of methods that have been used to date for improving reactive recovery responses during walking (using spatiotemporal or biomechanical parameters) and reducing falls (defined using the number of daily life or laboratory-induced falls after exposure to the perturbation paradigms) among healthy older adults.

\section{Methods}

A systematic search of PubMed, Web of Science, MEDLINE and CINAHL databases was conducted with sets of terms relating to gait (gait, locomotion, walk, walking), perturbations (agility, balance loss, dynamic balance, dynamic stability, perturb*, slip*, surface translation, trip, tripping, waist pull), ageing (age, ageing, aged, aging, elderly, old, older, senior), and adaptation or training (adaptation, adaptive, adjustments, exercise, rehabilitation, repeated, repetition, responses, task, training). An additional file detailing the search terms for each database is available [Additional file 1]. This broad range of terms was used due to the large variance in terminology used in the literature to describe the tasks and underlying mechanisms of interest, as well as the fact that reactive stability tasks are not always specifically described in the titles and abstracts of larger intervention studies. The initial search was conducted on December $16^{\text {th }} 2015$, with the final check for recent literature conducted on May 18 ${ }^{\text {th }}$ 2016. Two of the authors independently screened titles, abstracts and full texts for inclusion. It was planned that disagreements regarding inclusion would be discussed and when an agreement could not be reached, a third author would be consulted, but this was not required as the two authors agreed on the articles included and excluded. Inclusion criteria stipulated that the studies were conducted with healthy participants with a mean age of 60 years or older, that the studies applied repeated mechanical perturbations of an unpredictable or unannounced nature during walking, and that reactive recovery responses to gait perturbations or the incidence of laboratory or daily life falls were recorded. The inclusion process for this review, including the number of articles excluded at each stage can be seen in Figure 1. Once the articles to be included were finalised, a risk of bias assessment using the Physiotherapy Evidence Database (PEDro) Scale (Maher et al., 2003, de Morton, 2009) was carried out for each article. The PEDro website was consulted and when scores for the included articles were 
available, these were used. For the remaining articles, two authors independently scored the articles and then compared and discussed the scores before finalising them. Following this, the level of evidence was determined (as described by Teasell et al. (2007)) for each type of perturbation used in the included studies.

\section{Results}

\section{Systematic Search Results}

The complete search and inclusion process can be seen in Figure 1. The search yielded 5223 records, which was reduced to 3332 after duplicates were removed. The title screening excluded 3113 records, after which the remaining 219 articles' abstracts were assessed for inclusion. 27 full texts were then assessed and nine articles met all inclusion criteria. The reasons for exclusion at the full text screening stage can be found in Figure 1.

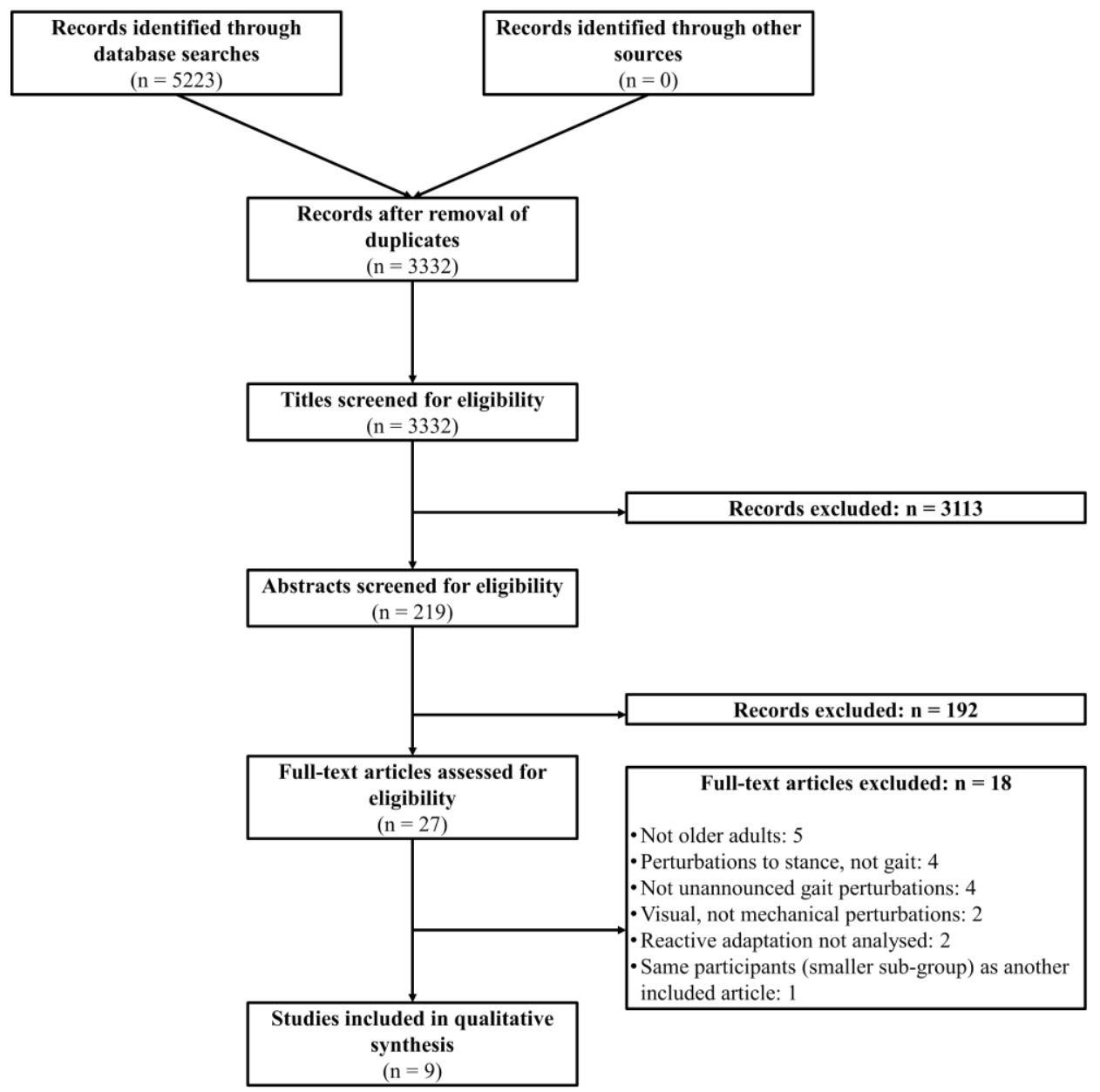

Fig. 1: Flowchart of systematic search and article inclusion and exclusion process. 


\section{Summary of Included Studies}

The systematic search and inclusion process yielded nine studies that met all inclusion criteria. A summary of the participants, the perturbation paradigms, the assessment methods for reactive recovery responses and falls incidence, and the main results of the included articles are reported in Table 1. In these nine studies, moveable floor platforms (Pai et al., 2010, Bhatt et al., 2012, Parijat and Lockhart, 2012, Pai et al., 2014a, Pai et al., 2014b), ground surface compliance changes (Bierbaum et al., 2010, Bierbaum et al., 2011) and treadmill belt accelerations or decelerations (Sakai et al., 2008, Lurie et al., 2013) were used to perturb the gait of older adults. Eight of the studies used a single session of perturbations (Sakai et al., 2008, Bierbaum et al., 2010, Pai et al., 2010, Bierbaum et al., 2011, Bhatt et al., 2012, Parijat and Lockhart, 2012, Pai et al., 2014a, Pai et al., 2014b), with two studies using multiple sessions (Bhatt et al., 2012, Lurie et al., 2013). Eight of the studies reported improvement in some measure of the reactive recovery response to the perturbations (Sakai et al., 2008, Bierbaum et al., 2010, Pai et al., 2010, Bierbaum et al., 2011, Bhatt et al., 2012, Parijat and Lockhart, 2012, Lurie et al., 2013, Pai et al., 2014b). Four studies observed a reduction in the percentage of laboratory falls from the pre- to post-perturbation experience measurements (Pai et al., 2010, Bhatt et al., 2012, Parijat and Lockhart, 2012, Pai et al., 2014b) and two studies reported a reduction of daily life falls (Lurie et al., 2013, Pai et al., 2014a). While most of the included studies were conducted with healthy, community dwelling older adults, it is important to note that Lurie et al. (2013) included participants referred for gait and balance training by their primary care provider, but no specific diagnoses or conditions were mentioned.

\section{Perturbation Paradigms}

In the nine included studies, moveable floor platforms used for simulating slips were the most commonly used perturbation type (Pai et al., 2010, Bhatt et al., 2012, Parijat and Lockhart, 2012, Pai et al., 2014a, Pai et al., 2014b). In these five studies, participants walked at self-selected speeds (Pai et al., 2010, Bhatt et al., 2012, Parijat and Lockhart, 2012, Pai et al., 2014a, Pai et al., 2014b). In four of these five studies, the platforms could freely slide up to $90 \mathrm{~cm}$ whereas in the other study, the velocity was controlled (limited to a velocity of $1.2 \mathrm{~m} / \mathrm{s}$ and maximum acceleration of $\left.20 \mathrm{~m} / \mathrm{s}^{2}\right)$ and the maximum displacement was only $30 \mathrm{~cm}$ (Parijat and Lockhart, 2012). In the $90 \mathrm{~cm}$ sliding platform condition, the platforms were unlocked at foot touchdown, detected by the force plates, using a mechanical locking mechanism. The papers describe the platforms as low friction and while exact velocity of the platforms' slides are not reported (these will have varied due to different walking velocities and limb or body configurations at touchdown), the percentage of older adults who fell during the first slip ranged from $42.5 \%$ to $56 \%$, indicating the reasonably high magnitude and impact of the perturbation (Pai et al., 2010, Bhatt et al., 2012, Pai et al., 2014a, Pai et al., 2014b). These four studies used a protocol containing 24 slips in 37 walking trials, whereas Parijat and Lockhart (2012) applied 12 slips in 24 walking trials. 


\section{1}

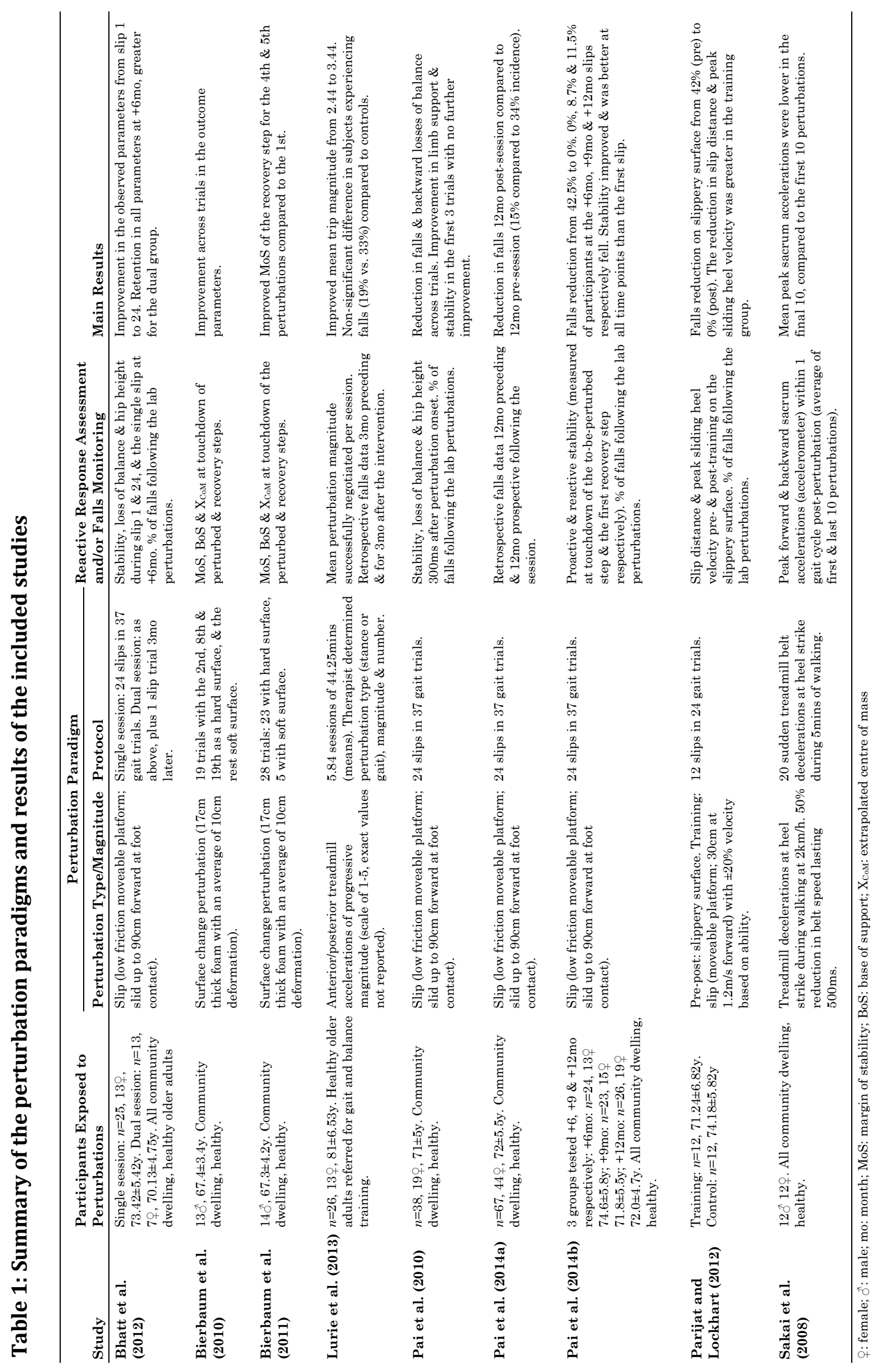


Ground surface compliance changes were applied in two of the included studies (Bierbaum et al., 2010, Bierbaum et al., 2011). This perturbation consisted of a section of the walkway that could be replaced with a soft element without a visible difference to the normal hard surface. The soft element was composed of a $17 \mathrm{~cm}$ thick piece of foam with an average deformation of about $10 \mathrm{~cm}$ for the participants (Bierbaum et al., 2010, Bierbaum et al., 2011). In both studies, the measurements began with three baseline walking trials where the hard element was used (Bierbaum et al., 2010, Bierbaum et al., 2011). In the first study, 19 walking trials were conducted after baseline, where only the $2^{\text {nd }}, 8^{\text {th }}$ and $19^{\text {th }}$ trials used the hard surface (Bierbaum et al., 2010). While this paradigm was used to assess predominantly predictive, feedforward locomotor adaptation during repeated soft surface trials, we may assume that reactive, feedback-driven locomotor adaptations played a role in the first four soft surface trials, as the participants were not aware which surface would be used on a given trial. The second study was, however, specifically designed to assess reactive adaptation, where 28 trials in total were conducted, with only five soft surface trials interspersed throughout the hard surface trials (Bierbaum et al., 2011). Improvements in stability control were seen during both the first four soft surface trials in the first study (Bierbaum et al., 2010) and by the fourth soft surface trial of the second study (Bierbaum et al., 2011). However, as these studies did not report numbers of lab or daily life falls, it is difficult to determine the impact such a perturbation paradigm could have, when used as training, on daily life falls incidence. In these studies, walking speed was set at $60 \%$ of walk-to-run velocity, based on walking trials conducted before the perturbation trials (Bierbaum et al., 2010, Bierbaum et al., 2011).

Finally, two studies used treadmill belt accelerations or decelerations (Sakai et al., 2008, Lurie et al., 2013) to perturb the gait of their participants. Sakai et al. (2008) used a deceleration perturbation during walking at $2 \mathrm{~km} / \mathrm{h}$, resulting in a $50 \%$ reduction in belt speed at heel strike over $0.5 \mathrm{~s}$. This perturbation was applied 20 times during five minutes of walking. Lurie et al. (2013) used a combination of treadmill belt accelerations and decelerations during both stance and gait, but did not report the exact number of perturbations used in their study, as the perturbation type (stance or gait), magnitude (scale of 1-5; no velocity/acceleration values reported) and number was determined by a physical therapist for each participant individually. The authors determined the perturbation magnitude based on the treadmill belt pulse peak velocity, elapsed time to peak velocity, elapsed time during which the peak velocity was maintained, and time required to decelerate the treadmill belt to zero velocity, but these values or ranges were not reported (Lurie et al., 2013). On average, the participants completed approximately six sessions of 45 minutes and the sessions were progressive in terms of perturbation magnitude, based on the physical therapist's judgement (Lurie et al., 2013).

\section{Reactive Recovery Responses and Falls Reduction in the Included Studies}

In the included studies, perturbation paradigms including four (Bierbaum et al., 2010), five (Bierbaum et al., 2011), 12 (Parijat and Lockhart, 2012) and 24 (Pai et al., 2010, Bhatt et al., 2012, Pai et al., 2014a, Pai et al., 2014b) perturbations led to improved reactive recovery responses to the disturbances, with one study showing transfer to another 
perturbation task (Parijat and Lockhart, 2012). Lurie et al. (2013) reported that the mean perturbation magnitude of successfully negotiated perturbations significantly increased from the first to final session. Sakai et al. (2008) found that the mean peak anteroposterior acceleration (determined using an accelerometer attached to the sacrum) was significantly reduced in the final 10 perturbations, in comparison to the first 10, indicating an improved reactive recovery response. Four of the included studies reported a reduction in the percentage of participants who fell from $42.5 \%$ to $56 \%$ during the first perturbation, to $0 \%$ following 12 (Parijat and Lockhart, 2012) and 24 (Pai et al., 2010, Bhatt et al., 2012, Pai et al., 2014b) perturbations, with one of the 24 perturbation studies reporting a reduction to $5 \%$ after only five perturbations (Pai et al., 2010). Pai et al. (2014a) reported a 50\% reduction in daily life falls in the 12 months following the perturbation session. Lurie et al. (2013) reported that their intervention group participants experienced fewer falls (19\% vs. 33\%) and fewer falls that led to injuries (8\% vs. 18\%) in comparison to the control group, but these were not statistically significant as the study was not powered to detect changes in falls incidence.

PEDro Scale scores of the included articles can be found in Table 2. The mean score of all articles was 3.33, with only three studies receiving a score of four or higher. The levels of evidence for the different perturbation paradigms of the included studies are presented in Table 3. Based on the definitions provided by Teasell et al. (2007), strong evidence exists only for moveable platform perturbations, as two or more RCTs have demonstrated beneficial effects of experiencing this type of perturbation on reactive recovery responses and falls incidence. Moderate evidence exists for treadmill-based perturbations as only one RCT has reported beneficial effects to date. Finally, the level of evidence for surface change perturbations was limited, as no RCTs have been conducted using this type of gait perturbation.

Table 2: PEDro Scale scores for individual studies included in this review

\begin{tabular}{lcccccccccccc}
\hline \multicolumn{1}{c}{ Study } & \multicolumn{1}{c}{ PEDro Scale Item } \\
\cline { 2 - 12 } & $\mathbf{1 a}^{\mathrm{a}}$ & $\mathbf{2}$ & $\mathbf{3}$ & $\mathbf{4}$ & $\mathbf{5}$ & $\mathbf{6}$ & $\mathbf{7}$ & $\mathbf{8}$ & $\mathbf{9}$ & $\mathbf{1 0}$ & $\mathbf{1 1}$ & Total \\
\hline Bhatt et al. (2012)* & Yes & 1 & 0 & 1 & 0 & 0 & 0 & 0 & 0 & 1 & 1 & 4 \\
Bierbaum et al. (2010) & No & 0 & 0 & 0 & 0 & 0 & 0 & 0 & 0 & 1 & 1 & 2 \\
Bierbaum et al. (2011) & No & 0 & 0 & 0 & 0 & 0 & 0 & 0 & 0 & 1 & 1 & 2 \\
Lurie et al. (2013)* & Yes & 1 & 1 & 1 & 0 & 0 & 0 & 1 & 0 & 1 & 1 & 6 \\
Pai et al. (2010) & Yes & 0 & 0 & 0 & 0 & 0 & 0 & 0 & 0 & 1 & 1 & 2 \\
Pai et al. (2014a)* & Yes & 1 & 0 & 1 & 0 & 0 & 0 & 0 & 1 & 1 & 1 & 5 \\
Pai et al. (2014b) & Yes & 0 & 0 & 1 & 0 & 0 & 0 & 0 & 0 & 1 & 1 & 3 \\
Parijat and Lockhart (2012) & No & 1 & 0 & 0 & 0 & 0 & 0 & 0 & 0 & 1 & 1 & 3 \\
Sakai et al. (2008) & No & 0 & 0 & 0 & 0 & 0 & 0 & 1 & 1 & 0 & 1 & 3 \\
\hline
\end{tabular}

PEDro Scale Items: 1: Eligibility criteria were specified; 2: Subjects were randomly allocated to groups; 3: Allocation was concealed; 4: The groups were similar at baseline regarding the most important prognostic indicators; 5 : There was blinding of all subjects; 6 : There was blinding of all therapists who administered the therapy; 7 : There was blinding of all assessors who measured at least one key outcome; 8: Measures of at least one key outcome were obtained from more than 85\% of the subjects initially allocated to groups; 9: All subjects for whom outcome measures were available received the treatment or control condition as allocated or, where this was not the case, data for at least one key outcome was analysed by "intention to treat"; 10: The results of between-group statistical comparisons are reported for at least one key outcome; 11: The study provides both point measures and measures of variability for at least one key outcome. Ratings: No/unclear $=0$, Yes $=1$.

a: Not included in total score.

*: Scores obtained from PEDro website (http://www.pedro.org.au). 
Table 3: Level of evidence per perturbation type for improving reactive responses and/or falls risk

\begin{tabular}{lccccl}
\hline & \multicolumn{2}{c}{ Studies Reporting Beneficial } \\
Effects & \multicolumn{2}{c}{$\begin{array}{c}\text { Negative Studies } \\
\text { (with sufficient } \\
\text { power) }\end{array}$} & Level of \\
\cline { 2 - 5 } Perturbation Type & RCTs & Non-RCTs & RCTs & Non-RCTs & Evidence* \\
\hline Moveable floor platform & 3 & 2 & 0 & 0 & Strong \\
Treadmill & 1 & 1 & 0 & 0 & Moderate \\
(acceleration/deceleration) & 0 & 2 & 0 & 0 & Limited \\
Surface Change & & 2 & 0 & 0 \\
\hline
\end{tabular}

*Level of evidence based on Teasell et al. [61]: Strong Evidence: Two or more RCTs with PEDro scores of 4 or higher; Moderate Evidence: One RCT with a PEDro score of 4 or higher; Limited Evidence: At least one non-RCT (i.e. prospective or retrospective controlled trials, single group studies etc.).

\section{Discussion}

The aim of this systematic review was to determine the variety, characteristics and effectiveness of methods that have been used to date for improving reactive recovery responses during walking and reducing falls among healthy older adults. To achieve this, a systematic search for studies with healthy older adults that applied unexpected mechanical disturbances during walking and assessed changes in reactive recovery responses or falls incidence was conducted. After screening, nine articles met the inclusion criteria. Moveable floor platforms (Pai et al., 2010, Bhatt et al., 2012, Parijat and Lockhart, 2012, Pai et al., 2014a, Pai et al., 2014b), ground surface compliance changes (Bierbaum et al., 2010, Bierbaum et al., 2011) and treadmill belt accelerations or decelerations (Sakai et al., 2008, Lurie et al., 2013) have been used to perturb the gait of older adults with the aim of stimulating adaptations in the reactive response. Eight of the nine studies reported improvement in the reactive recovery response (Sakai et al., 2008, Bierbaum et al., 2010, Pai et al., 2010, Bierbaum et al., 2011, Bhatt et al., 2012, Parijat and Lockhart, 2012, Lurie et al., 2013, Pai et al., 2014b), four studies reported a reduction in laboratory falls (Pai et al., 2010, Bhatt et al., 2012, Parijat and Lockhart, 2012, Pai et al., 2014b) and two studies reported a reduction in daily life falls (Lurie et al., 2013, Pai et al., 2014a). As well as the range of perturbation types, the magnitude and frequency of the perturbations varied between the studies.

Regarding the number and magnitude of the moveable platform perturbations, one study (Parijat and Lockhart, 2012) applied 50\% fewer perturbations with a smaller magnitude in comparison to the other studies utilizing platforms (Pai et al., 2010, Bhatt et al., 2012, Pai et al., 2014a, Pai et al., 2014b). Despite this difference in magnitude and number of perturbations, all studies found statistically significant improvements in various measures, including a reduction in the number of trials where participants required support from the safety harness (classed as falls). Combined with the fact that one of the studies reported that the majority of the improvements occurred within the first five perturbation trials (Pai et al., 2010), it appears that healthy older adults can benefit from experiencing only a few moveable platform perturbations. This could be important for future research and application in clinical settings, as this implies that the minimum effective dose of such perturbations could be very low. However, more research is needed to determine if such a low number of perturbations would also yield long term benefits, in addition to these acute benefits seen in the lab. 
In order to fully understand and interpret the results of gait perturbation studies, it is important to consider how walking speed and perturbation magnitudes were controlled or scaled based on the participants included. Concerning the studies included in this review, the five studies using moveable floor platforms had participants walking at self-selected speeds (Pai et al., 2010, Bhatt et al., 2012, Parijat and Lockhart, 2012, Pai et al., 2014a, Pai et al., 2014b). Other than walking speed, no scaling or standardisation of the paradigm based on the participants was conducted, apart from an increase or decrease of $20 \%$ platform slip velocity in the study of Parijat and Lockhart (2012) which was based on participants' performance during the session. In the two ground surface compliance change perturbation studies, walking speed was set at $60 \%$ of walk-to-run velocity, based on walking trials conducted before the main measurements and the perturbation itself was not adjusted based on the participants (Bierbaum et al., 2010, Bierbaum et al., 2011). Concerning the two treadmill-based paradigms, Sakai et al. (2008) used a set walking speed of $2 \mathrm{~km} / \mathrm{h}$ for all participants with no changes in the perturbation, while Lurie et al. (2013) used individualised walking speeds and perturbation intensities based on the abilities of the participants (values or ranges of speeds and magnitudes were not reported) (Lurie et al., 2013). Due to individual differences in locomotor capacities, using the same walking speed for all participants (as in: Sakai et al., 2008) may lead to some being more challenged than others by the perturbations applied. This may lead to floor or ceiling effects in the adaptation to perturbations, which may be particularly problematic when comparing groups of different locomotor capacities (McCrum et al., 2016b). In the same manner, using the same (or similar) perturbations for participants with different capacities (Sakai et al., 2008, Bierbaum et al., 2010, Pai et al., 2010, Bierbaum et al., 2011, Bhatt et al., 2012, Pai et al., 2014a, Pai et al., 2014b) may lead to similar issues, as one individual or group may require relatively more substantial adaptation than others to maintain stability. When using a self-selected (as in: Pai et al., 2010, Bhatt et al., 2012, Pai et al., 2014a, Pai et al., 2014b) or individually standardised (as in: Bierbaum et al., 2010, Bierbaum et al., 2011) walking speed, faster walking speeds may make stability recovery more difficult following a perturbation, compared to slower speeds, due to a higher forward velocity, and therefore a reduced margin of stability in the forward direction (Süptitz et al., 2012). How the interaction of walking speed and perturbation magnitude influences reactive recovery responses and adaptation to perturbations in different age and patient groups remains a question for future studies.

As well as the type, number and magnitude of perturbations, perturbation direction may be an important feature of such paradigms with regard to daily life falls reduction. In the studies included in this review, perturbations were mostly applied in anterior or posterior directions. This is noteworthy, as it is well documented that mediolateral stability declines with age (Maki et al., 2000, Mille et al., 2005, Schrager et al., 2008, Mille et al., 2013, Singer et al., 2016) and is related to falls incidence in older adults (Maki et al., 1994, Lord et al., 1999, Hilliard et al., 2008, Fujimoto et al., 2015). Additionally, there is evidence to suggest that adaptations to perturbations in one plane of motion do not necessarily transfer and benefit stability control in other planes of motion (Dijkstra et al., 2015, Martelli et al., 2016, Peterson et al., 2016). Regarding the type and direction of perturbations used to stimulate adaptation in the reactive recovery responses, it has been 
previously suggested that, due to the diversity of perturbations that can occur in daily life, it may be more effective to train the mechanisms of stability recovery, rather than focus on specific perturbations (Arampatzis et al., 2011, Bierbaum et al., 2013). Such an approach (whereby multidirectional stepping and counter rotating mechanisms to maintain balance are exercised) has been shown to result in an improvement in stability recovery following lab-based perturbations (Aragao et al., 2011, Arampatzis et al., 2011, Bierbaum et al., 2013). However, no study has yet looked at the effects of such an intervention on daily life falls incidence in older adults. Furthermore, if particular gait perturbation paradigms, like those described in this review, would also result in an improvement in these mechanisms, there may not be any reason to suspect a less positive outcome on daily life falls.

The mean PEDro Scale score of the included studies was 3.33, with only three studies receiving higher scores of four, five and six (Table 2). However, it should be kept in mind that blinding of the participants and staff members conducting such experiments or training is difficult, meaning that points five and six of the PEDro scale will generally not be met by such studies. Regarding the levels of evidence determined for the perturbation paradigms of the studies included in this review, it is important to note the relatively low number of RCTs conducted thus far, especially with paradigms other than moveable floor platforms. Until more RCTs are conducted with varying perturbation paradigms, concrete conclusions regarding the beneficial effects of different perturbations are difficult to make. Despite the strong evidence for moveable platform perturbations, based on the definitions provided by Teasell et al. (2007), a number of advantages of treadmill setups should be highlighted. The first relates to the predictability of the perturbations applied in a gait lab setup with overground walking, in comparison to treadmill walking. In overground setups, the location of the perturbation on the walkway is usually constant, which means that even if the perturbation is not applied in every trial, the participant may make predictive, feedforward adaptations in their gait after identifying the location of the potential perturbation, facilitating better performance and adaptation. Shapiro and Melzer (2010) have previously highlighted similar issues related to the same perturbation direction being used for all trials. In contrast, treadmill setups do not face this location issue, as accelerations or decelerations can be applied at any time during continuous walking, making it more difficult for participants to anticipate perturbations. As well as this, depending on the setup, perturbations in multiple directions could be used. Furthermore, treadmill perturbation setups may be more feasible in clinical settings (Lurie et al., 2013) due to the smaller space required, in comparison to a gait lab and walkway. However, the type and magnitude of perturbations that can be applied may be limited by the size and capabilities of the treadmill.

While this systematic review found three main types of perturbations that have been used to examine and stimulate the reactive adaptation of gait in older adults, there are a number of other gait perturbation paradigms reported in the literature. These were excluded from this review due to the participant population used (i.e. not older adults), or that reactive adaptation was not analysed in the studies. Cable trip systems, in conjunction with either treadmill or overground walking have been used effectively on multiple occasions to analyse gait stability and adaptation in multiple participant groups 
(Süptitz et al., 2012, McCrum et al., 2014, Senden et al., 2014, McCrum et al., 2016a, McCrum et al., 2017a). Another method for initiating tripping responses is the use of objects popping up from the ground, inhibiting the swing phase of gait (Pijnappels et al., 2005b, Pijnappels et al., 2005c). As well as tripping methods, a few different methods have been used to trigger slipping responses in addition to the moveable platforms from the included studies described above. The slip perturbation used to test the participants in the study of Parijat and Lockhart (2012) could also be used in a repetitive manner to stimulate adaptation. Additionally, different levels of shoe/floor friction using different materials have been used (Heiden et al., 2006). As well as trips and slips, a number of various waist push and pull methods have been applied during gait to analyse mediolateral stability in particular (Hof et al., 2010, Sturnieks et al., 2013, Martelli et al., 2016). Finally, perturbations involving sudden surface height changes (van der Linden et al., 2007, van der Linden et al., 2009, Müller et al., 2015) or multiple changes in surface tilt, height and position (Nashner, 1980) have been employed. The majority of these perturbations have been used to investigate some specific characteristic of gait stability or adaptability but few have been used for the purpose of training. Therefore, further research is needed before recommending these perturbations for training purposes among older adults.

One potential limitation of this review (and the studies included) is that it is difficult to determine if the responses to the perturbations were fully reactive in nature. In most movements, an interplay exists between reactive and predictive adaptations (Pavol et al., 2004). In order to reduce the influence of predictive adaptations, two steps can be taken. Firstly, the degree of predictability of the perturbations must be kept to a minimum (e.g. using catch trials, random timing etc.), and secondly, attempts can be made to assess pre-perturbation movement to assess if predictive adjustments are being made. As described above, the setup may also affect the predictability of the perturbations and results should be interpreted with this in mind. One study that we know of (Pater et al., 2015) has applied a truly unexpected perturbation, albeit with young participants, where participants were under the impression that they were taking part in a normal gait analysis and were subsequently perturbed. The effects of this perturbation were markedly greater compared to the more common situation where the participants knew that they would be perturbed at some point during the trial (Pater et al., 2015). However, such a procedure has not been conducted with older adults.

It is important to note that in this review, we focussed on gait, as opposed to stance perturbation paradigms. As detailed in the introduction, perturbations applied during gait are theoretically more task specific to daily life falls among healthy older adults than stance perturbations, as most falls occur during walking in this population (Tinetti et al., 1988, Berg et al., 1997, Li et al., 2006, Kelsey et al., 2012, Robinovitch et al., 2013). Additionally, forward velocity during gait may make stability recovery following a perturbation more difficult, as an increased walking speed, and thereby higher forward centre of mass velocity, results in a lower anterior margin of stability (Süptitz et al., 2012). However, a decrease in falls incidence has been shown after four sessions of perturbations to stance in healthy older adults (Rosenblatt et al., 2013), indicating that stance perturbations may also be beneficial. To our knowledge, a direct comparison of the effectiveness of stance versus gait perturbations for falls reduction among older adults has 
not been made, and it is not known how repetition of either gait or stance perturbations would benefit performance of the other. Stance perturbations have often been applied in patient populations (for example: Suteerawattananon et al., 2002, Protas et al., 2005, Smania et al., 2010), possibly due to practical reasons (simpler setup, easier quantification of stability) or perhaps due to the relatively lower demand of the tasks, in comparison to gait perturbations. This suggests that a progression could be made from stance to gait perturbations in clinical settings.

\section{Conclusions}

To date, a range of perturbation paradigms (moveable floor platforms, ground surface compliance changes and treadmill belt accelerations or decelerations) have been used to perturb older adults while walking. As well as the range of perturbation types that have been applied, there is huge variation among studies in the number and magnitude of the perturbations. The fact that the majority of studies report improvements in participants' ability to maintain stability following exposure to the perturbation paradigms is in one sense promising, as benefits appear to be produced from many different paradigms, but this restricts our understanding of the underlying mechanisms of improvement and what components of the paradigms are responsible for the improvements. The effects of perturbation type, magnitude and number on the extent of adaptation of the reactive recovery responses and the retention of such adaptations over longer time periods should be investigated in future research. This may lead to more efficient and effective perturbation paradigms and to information regarding the minimum effective dose for falls incidence reduction among healthy older adults. 


\section{Supplementary Material}

Additional file 1: Search terms in PubMed, Web of Science, MEDLINE and CINAHL

PubMed Search on 16/12/2015:

((((gait OR walking OR walk OR locomotion)) AND (Perturb* OR trip OR tripping OR slip* OR surface translation OR balance loss OR agility OR dynamic balance OR dynamic stability OR waist pull)) AND (training OR exercise OR adaptation OR adaptive OR repeated OR repetition OR rehabilitation OR task OR responses OR adjustments)) AND (Age OR ageing OR aging OR aged OR elderly OR old OR older OR senior)

Search returned: 1911 results

Web of Science on 16/12/2015:

(TS=(gait OR walking OR walk OR locomotion) AND TS=(Perturb* OR trip OR tripping OR slip* OR surface translation OR balance loss OR agility OR dynamic balance OR dynamic stability OR waist pull) AND TS=(training OR exercise $\mathrm{OR}$ adaptation $\mathrm{OR}$ adaptive $\mathrm{OR}$ repeated $\mathrm{OR}$ repetition OR rehabilitation OR task OR responses OR adjustments) AND TS=(Age OR ageing OR aging OR aged OR elderly OR old OR older OR senior)) OR (TI=(gait OR walking OR walk OR locomotion) AND TI=(Perturb* OR trip OR tripping OR slip* OR surface translation OR balance loss OR agility OR dynamic balance OR dynamic stability OR waist pull) AND TI=(training OR exercise OR adaptation OR adaptive OR repeated OR repetition OR rehabilitation OR task OR responses OR adjustments) AND TI=(Age OR ageing OR aging OR aged OR elderly OR old OR older OR senior))

Search returned: 1454 results

\section{MEDLINE on 16/12/2015:}

((gait or walking or walk or locomotion) and (Perturb* or trip or tripping or slip* or surface translation or balance loss or agility or dynamic balance or dynamic stability or waist pull) and (training or exercise or adaptation or adaptive or repeated or repetition or rehabilitation or task or responses or adjustments) and (Age or ageing or aging or aged or elderly or old or older or senior)).mp. [mp=title, abstract, original title, name of substance word, subject heading word, keyword heading word, protocol supplementary concept word, rare disease supplementary concept word, unique identifier]

Search returned: 970 results

CINAHL on 16/12/2015:

TX ( gait OR walking OR walk OR locomotion ) AND TX ( Perturb* OR trip OR tripping OR slip* OR surface translation OR balance loss OR agility OR dynamic balance OR dynamic stability OR waist pull ) AND TX ( training OR exercise OR adaptation OR adaptive OR repeated OR repetition 
OR rehabilitation OR task OR responses OR adjustments) AND TX (Age OR ageing OR aging OR aged OR elderly OR old OR older OR senior )

\section{Search returned: 888 results}

In total: 5223 records

After removal of duplicates: 3332

After title screening: 219

After abstract screening: 27

After full text screening: 9 


\section{2:}

\section{Stability-normalised walking speed: a new approach for human gait perturbation research}

Christopher McCrum, Paul Willems, Kiros Karamanidis, Kenneth Meijer

Published as:

MCCRUM, C., WILLEMS, P., KARAMANIDIS, K. \& MEIJER, K. 2019. Stability-normalised walking speed: A new approach for human gait perturbation research. J Biomech, 87, 48-53. doi:

10.1016/j.jbiomech.2019.02.016

Open access preprint version at bioRxiv. doi: 10.1101/314757

(C) 2019 Elsevier Ltd. All rights reserved. 


\begin{abstract}
In gait stability research, neither self-selected walking speeds, nor the same prescribed walking speed for all participants, guarantee equivalent gait stability among participants. Furthermore, these options may differentially affect the response to different gait perturbations, which is problematic when comparing groups with different capacities. We present a method for decreasing inter-individual differences in gait stability by adjusting walking speed to equivalent margins of stability (MoS). Eighteen healthy adults walked on a split-belt treadmill for two-minute bouts at $0.4 \mathrm{~m} / \mathrm{s}$ up to $1.8 \mathrm{~m} / \mathrm{s}$ in $0.2 \mathrm{~m} / \mathrm{s}$ intervals. The stability-normalised walking speed $(\mathrm{MoS}=0.05 \mathrm{~m})$ was calculated using the mean MoS at touchdown of the final 10 steps of each speed. Participants then walked for three minutes at this speed and were subsequently exposed to a treadmill belt acceleration perturbation. A further 12 healthy adults were exposed to the same perturbation while walking at $1.3 \mathrm{~m} / \mathrm{s}$ : the average of the previous group. Large ranges in MoS were observed during the prescribed speeds $(6-10 \mathrm{~cm}$ across speeds) and walking speed significantly $(P<0.001)$ affected MoS. The stability-normalised walking speeds resulted in MoS equal or very close to the desired $0.05 \mathrm{~m}$ and reduced between-participant variability in MoS. The second group of participants walking at $1.3 \mathrm{~m} / \mathrm{s}$ had greater inter-individual variation in MoS during both unperturbed and perturbed walking compared to $12 \mathrm{sex}$, height and leg length-matched participants from the stability-normalised walking speed group. The current method decreases inter-individual differences in gait stability which may be beneficial for gait perturbation and stability research, in particular for studies on populations with different locomotor capacities.
\end{abstract}




\section{Introduction}

Mechanical perturbations have been used for decades to investigate the stability of human walking (Nashner, 1980, Berger et al., 1984, Quintern et al., 1985, Vilensky et al., 1999, Marigold and Patla, 2002) and are now frequently applied in falls prevention contexts (Pai and Bhatt, 2007, Mansfield et al., 2015b, Gerards et al., 2017). In gait perturbation studies, self-selected walking speeds (for example: Pai et al., 2014b) or a prescribed walking speed for all participants (for example: McCrum et al., 2016a) are commonly used, but each comes with drawbacks that complicate the interpretation of results.

A prescribed walking speed (for example, $1.5 \mathrm{~m} / \mathrm{s}$ for all participants) will not result in comparable stability for all participants. This is problematic when comparing groups with different capacities during a gait perturbation task, as the relative challenge of the task will vary. In such a situation, the difficulty in recovering stability following mechanical perturbations will be affected by the relative neuromuscular and biomechanical demands of the task. As well as the demand of recovering from one perturbation, the need for adaptation following repetition of a perturbation may be different. As a result, it is common to use the self-selected or preferred walking speed in gait perturbation research, but this can introduce other problems.

Having participants walk at their own self-selected speeds implies that there will be variation across participants, which is likely to be much greater when multiple groups with different locomotor capacities are involved. There is ample evidence that walking speed affects recovery strategy choice following slips (Bhatt et al., 2005) and trips (Krasovsky et al., 2014), the direction of balance loss following slipping (Smeesters et al., 2001) and differentially affects falls risk following tripping and slipping (Pavol et al., 1999, Bhatt et al., 2005, Espy et al., 2010). Gait stability at perturbation onset may also not be optimised at the self-selected speed and may differ across groups (Bhatt et al., 2005, Süptitz et al., 2012, Hak et al., 2013, Mademli and Arampatzis, 2014). For example, older adults walk with a lower safety factor than young adults at self-selected walking speeds (Mademli and Arampatzis, 2014) and reduce stability to benefit from centre of mass velocity when descending stairs; a potential compensation for reduced lower limb neuromuscular capacities (Bosse et al., 2012). Taken together, this evidence means that gait perturbation tasks could have very different effects across participants walking at their self-selected speeds, and it may be difficult to determine if group differences are true differences or artefacts of the above walking speed-related effects. These issues can be further confounded, as walking speed directly affects measures of dynamic gait stability using a centre of mass - base of support relationship model (Bhatt et al., 2005, Süptitz et al., 2012, Hak et al., 2013). Therefore, more sophistication in the choice of walking speed may be necessary for detailed study of reactive gait stability and adaptation processes.

Two possible solutions have been applied in previous gait perturbation studies. A Froude number (a dimensionless parameter) for walking speed (Hof, 1996) has been applied to normalise the walking speed based on leg length (Martelli et al., 2013, Aprigliano et al., 2016, Martelli et al., 2016, Aprigliano et al., 2017). Originally developed to analyse the dynamic similarity of differently sized boats (Vaughan and O'Malley, 2005), the Froude number has been applied for the purpose of comparing the gaits of different 
sizes and species of animals and results in dynamic similarity of the inverted pendulum motion in gait (Alexander, 1989, Alexander, 1991, Vaughan and O'Malley, 2005). However, while the inverted pendulum motion may be dynamically similar between participants, this normalisation based on leg length is not necessarily synonymous with a normalisation of gait stability, because factors such as individual differences in foot placement, posture, leg length to truck length ratio and internal properties of the neuromotor and neuromuscular systems are ignored. Task demand in such gait perturbation protocols (and most locomotor tasks) depends critically on these other factors and not only on the dimensions of the body; an 18-year-old and an 80-year-old with the same leg length are unlikely to be equally challenged by a gait perturbation while walking at the same speed. Two studies have used $60 \%$ of the walk-to-run velocity to normalise the speed to participants' walking-related neuromuscular capacities (Bierbaum et al., 2010, Bierbaum et al., 2011). However, this procedure did not lead to comparable stability during nonperturbed walking, with the margins of stability and the components of the margins of stability showing differences between the young and older subjects (Bierbaum et al., 2010, Bierbaum et al., 2011), again probably due to the fact that gait stability is not determined exclusively by the neuromuscular properties responsible for gait speed. As both existing normalisation methods are based on a single parameter, neither of which are the sole determinants of gait stability, one cannot expect equivalent gait stability among participants. Therefore, further attempts to tackle these issues are warranted (McCrum et al., 2016b, McCrum et al., 2017b).

Here, we present a new method for decreasing inter-individual differences in gait stability by normalising the walking speed based on gait stability. For this method we use the margins of stability (MoS) concept (Hof et al., 2005), one of the few well-defined and well-accepted biomechanical measures of mechanical stability of the body configuration during locomotion (Bruijn et al., 2013), useful for assessing changes in gait stability due to mechanical perturbations and balance loss. Additionally, we present results from a gait perturbation experiment comparing participants walking at their stability-normalised walking speed with participants walking all at the same prescribed speed.

\section{Methods}

\section{Participants}

Eighteen healthy adults participated in the first part of this study (eight males, 10 females; age: $24.4 \pm 2.5 y$; height: $174.9 \pm 7.4 \mathrm{~cm}$; weight: $74.6 \pm 15.2 \mathrm{~kg}$ ). Twelve healthy adults participated in the second part of the study (Table 1). The participants had no self-reported history of walking difficulties, dizziness or balance problems, and had no known neuromuscular condition or injury that could affect balance or walking. Informed consent was obtained and the study was conducted in accordance with the Declaration of Helsinki. The study protocol was approved by the Maastricht University Medical Centre medical ethics committee. 


\section{Setup and Procedures}

The Computer Assisted Rehabilitation Environment Extended (CAREN; Motekforce Link, Amsterdam, The Netherlands), comprised of a dual-belt force plate-instrumented treadmill (Motekforce Link, Amsterdam, The Netherlands; 1000Hz), a 12-camera motion capture system $(100 \mathrm{~Hz}$; Vicon Motion Systems, Oxford, UK) and a virtual environment that provided optic flow, was used for this study. A safety harness connected to an overhead frame was worn by the participants during all measurements. Five retroreflective markers were attached to anatomical landmarks (C7, left and right trochanter and left and right hallux) and were tracked by the motion capture system.

In the first part of the study (18 participants), the measurement sessions began with $60 \mathrm{~s}$ familiarisation trials of walking at $0.4 \mathrm{~m} / \mathrm{s}$ up to $1.8 \mathrm{~m} / \mathrm{s}$ in $0.2 \mathrm{~m} / \mathrm{s}$ intervals. After approximately five to ten minutes rest, single two-to-three-minute-long measurements were then conducted at the same speeds. Following these measurements, the stabilitynormalised walking speed was calculated. To determine the stability-normalised walking speed, the mean anteroposterior MoS (see below) at foot touchdown of the final 10 steps of each walking trial $(0.4 \mathrm{~m} / \mathrm{s}$ to $1.8 \mathrm{~m} / \mathrm{s})$ were taken and fitted with a second order polynomial function. For each participant, the speed resulting in $\mathrm{MoS}$ of $0.05 \mathrm{~m}$ was calculated. Based on our pilot testing, this value would result in walking speeds that would be possible for healthy adults of most ages (Bierbaum et al., 2010, Bierbaum et al., 2011, Süptitz et al., 2013). With certain populations, slower walking speeds would be required and then a greater MoS could be used. Participants then walked for three minutes at their stabilitynormalised walking speed, at the end of which, a gait perturbation was applied without warning. The perturbation consisted of an $80 \%$ increase in the right treadmill belt speed from the stability-normalised walking speed of the participant with a $3 \mathrm{~m} / \mathrm{s}^{2}$ acceleration, and thereby, we also normalised the magnitude of the perturbation to the already normalised walking speed. The acceleration began before touchdown of the to-beperturbed limb to ensure the belt was already at a higher speed when the foot touched down (triggered automatically by the D-Flow software of the CAREN, when the hallux marker of the to-be-perturbed limb became anterior to the stance limb hallux marker in the sagittal plane). The belt decelerated after toe-off of the perturbed limb.

In the second part of the study, 12 participants completed the same familiarisation protocol and then walked for three minutes at $1.3 \mathrm{~m} / \mathrm{s}$ (average stability-normalised walking speed of the 18 participants in the first part of the study). After this, they experienced the same treadmill belt acceleration perturbation. To compare these results with a matched sample, 12 participants from the first group of 18 were selected and matched specifically for sex, height and leg length to the participants in part two of the study (Table 1).

\section{Data Processing}

Marker tracks were filtered using a low pass second order Butterworth filter (zero-phase) with a $12 \mathrm{~Hz}$ cut-off frequency. Foot touchdown was detected using a combination of force plate (50N threshold) and foot marker data (Zeni et al., 2008). The anteroposterior MoS 
were calculated at foot touchdown as the difference between the anterior boundary of the base of support (anteroposterior component of the hallux marker projection to the ground) and the extrapolated centre of mass as defined by Hof et al. (2005), adapted for our reduced kinematic model based on Süptitz et al. (2013), as follows:

$$
\mathrm{X}_{\mathrm{CoM}}=\frac{\mathrm{P}_{\text {TroL }}+\mathrm{P}_{\text {TroR }}}{2}-\mathrm{P}_{\text {HalluxP }}+\frac{0.5\left(\frac{\mathrm{V}_{\text {TroL }}+\mathrm{V}_{\text {TroR }}}{2}+\mathrm{V}_{\mathrm{C} 7}\right)+\left|\mathrm{V}_{\text {Belt }}\right|}{\sqrt{\frac{\mathrm{g}}{\mathrm{L}_{\text {Ref }}}}}
$$

where $\mathrm{P}_{\text {TroL }}, \mathrm{P}_{\text {TroR }}$ and $\mathrm{P}_{\text {HalluxP }}$ are the trochanter and the posterior hallux marker anteroposterior positions respectively; $\mathrm{V}_{\text {TroL }}, \mathrm{V}_{\text {TroR }}$ and $\mathrm{V}_{\mathrm{C} 7}$ are the anteroposterior velocities of the trochanter and $\mathrm{C} 7$ markers respectively; $\mathrm{V}_{\mathrm{B} \text { elt }}$ is the treadmill belt velocity; $\mathrm{g}$ is gravitational acceleration $\left(9.81 \mathrm{~m} / \mathrm{s}^{2}\right)$; and $\mathrm{L}_{\mathrm{Ref}}$ is the reference leg length. This reduced kinematic model was previously shown to be suitable for assessing the MoS and it's components during unperturbed and perturbed treadmill walking in young, middle and older-aged healthy adults, with high correlations and no clear differences compared to a full kinematic model (Süptitz et al., 2013). Note that a large proportion of the CoM velocity is derived from the treadmill belt speed, potentially improving the accuracy compared with overground walking when the entire CoM velocity is derived from the markers. The MoS was calculated for: the final 10 steps of each set walking speed in the first part of the study; the mean MoS of the eleventh to second last step before each perturbation (Base); the final step before each perturbation (Pre); and the first recovery step following each perturbation (Post1).

\section{Statistics}

A mixed effects model for repeated measures with walking speed as a fixed effect and Tukey post hoc comparisons was used to confirm a walking speed effect on the MoS. To determine whether a normalisation of walking speed based on body dimensions would assume equivalent gait stability, Pearson correlations between the stability-normalised walking speeds and participants' height and leg length were conducted. A two-way repeated measures ANOVA with participant group (Stability-normalised walking speed $[$ Norm] and $1.3 \mathrm{~m} / \mathrm{s}$ ) and step (Base, Pre, Post1) as factors with post hoc Sidak's tests for multiple comparisons were used to determine between group differences in the MoS. Equivalence tests using $90 \%$ confidence intervals were used to confirm the similarity of the groups' demographics. Significance was set at $\alpha=0.05$. When sphericity was violated, a Greenhouse-Geisser correction was applied. Normality of the distributions was assessed with Q-Q plots. Analyses were performed using Prism version 8 for Windows (GraphPad Software Inc., La Jolla, California, USA). 


\section{Results and Discussion}

\section{Stability during unperturbed walking}

Walking speed significantly affected the $\operatorname{MoS}\left(\mathrm{F}_{[2.547,42.93]}=1485, P<0.0001, \hat{\varepsilon}=0.3638\right.$; Fig. 1) and Tukey's multiple comparisons tests revealed significant differences for each speed compared to all other speeds $(P<0.0001 ;$ Fig. 1$)$. These results agree with previous work (Bhatt et al., 2005, Süptitz et al., 2012, Hak et al., 2013). A range of MoS values were observed for each speed (approximately $6-10 \mathrm{~cm}$ ), even among these healthy participants, confirming some of the issues related to prescribed walking speeds in gait stability research discussed above. The strong relationship between walking speed and MoS also has relevance for clinical studies conducting self-paced gait measurements with an assessment of gait stability. Patients who improve in walking speed may demonstrate a reduction in MoS, which may not be reductions in the stability of the patients' gait per se, but simply an artefact of the improved walking speed.

The stability-normalised walking speeds (range from $1.22 \mathrm{~m} / \mathrm{s}$ to $1.51 \mathrm{~m} / \mathrm{s}$ with a mean $\pm \mathrm{SD}$ of $1.3 \pm 0.1 \mathrm{~m} / \mathrm{s}$ ) resulted in MoS very close to the desired outcome of $0.05 \mathrm{~m}$ (within one SD of the mean MoS for 15 of the 18 participants; Fig. 2A). The stability-normalised walking speed also reduced between-participant variability in MoS (as shown by the group level standard deviations; Fig. 2B). These combined results indicate that the stabilitynormalisation was successful in reducing between-participant differences in MoS during walking, even in a homogenous group of healthy young adults.

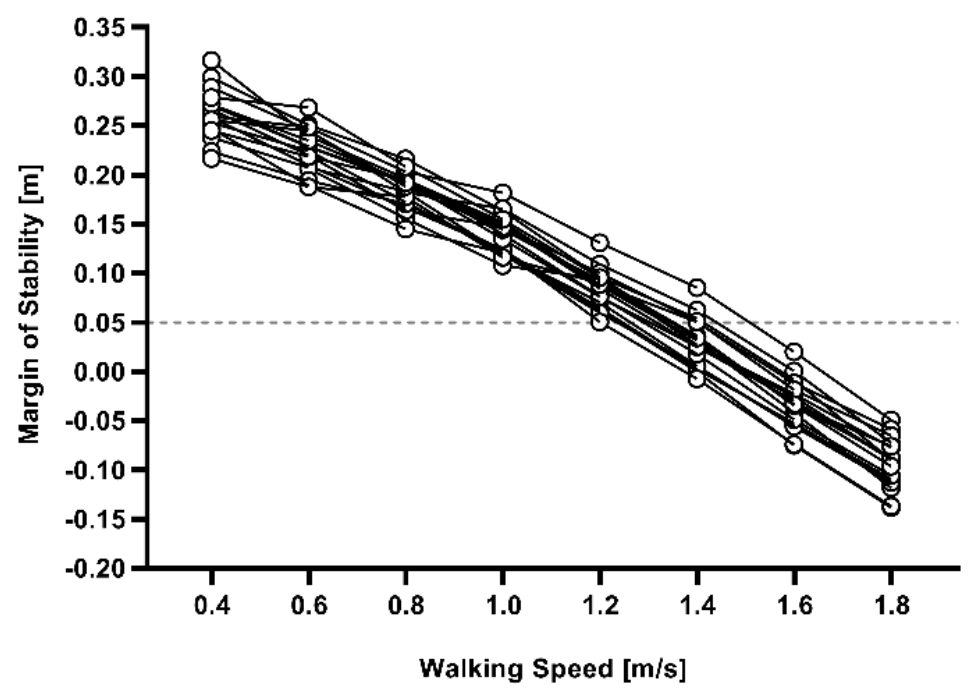

Fig. 1: Individual margins of stability at foot touchdown over the different walking speeds. The dashed line represents the margin of stability used to determine the stability-normalised walking speed. 

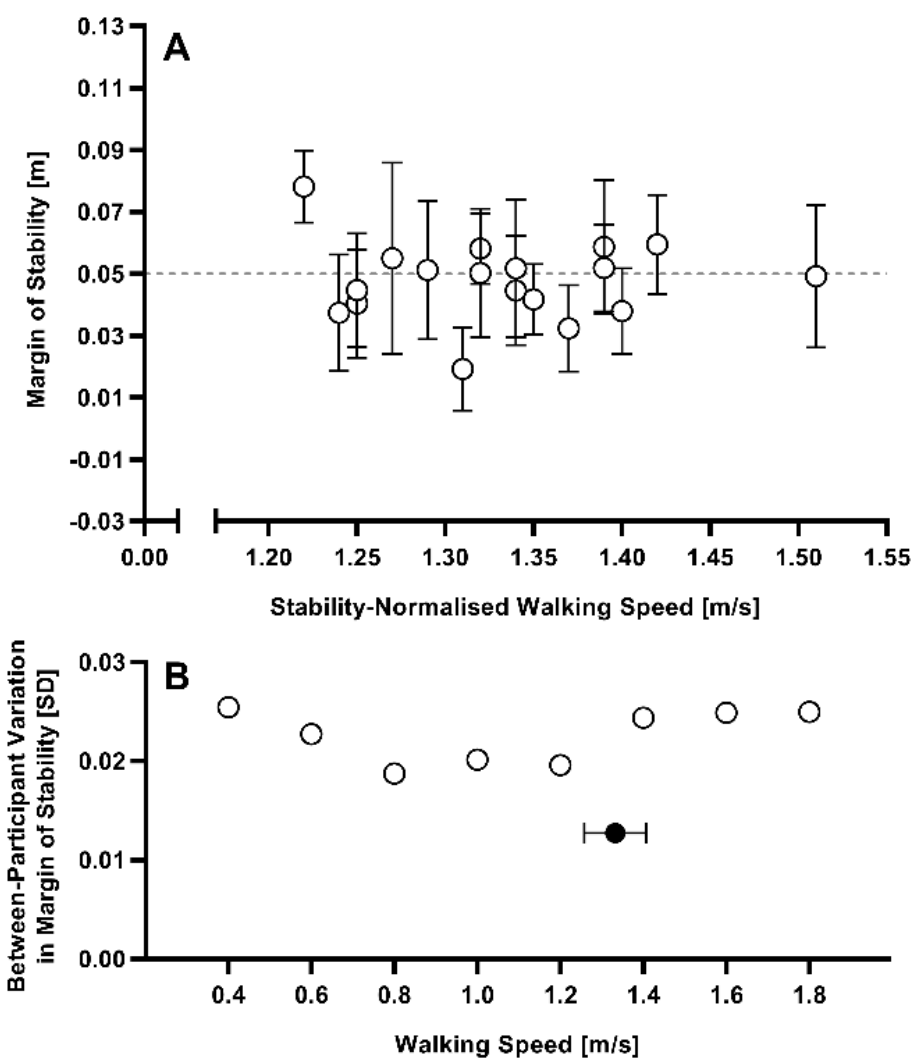

Fig. 2: A: Means and standard deviations of the margins of stability at touchdown of the final 10 steps at the stability-normalised walking speed for each individual participant. The desired MoS of $0.05 \mathrm{~m}$ at foot touchdown is indicated by the dashed line. B: The betweenparticipant variation in the margins of stability (standard deviation at group level) for the final 10 steps at each walking speed (the stability-normalised walking speed trials are indicated with the black circle; mean and standard deviation).

Small, non-significant correlations between the determined stability-normalised walking speeds and the participants' height and leg length were found (Fig. 3). The outcomes of our correlation analysis suggest that height and leg length did not significantly affect the calculation of stability-normalised walking speed, suggesting that a normalisation of walking speed based on body dimensions does not assume equivalent gait stability, at least not when assessed by the MoS concept.

\section{Stability during perturbed walking}

For the second part of the study, the 12 participants were successfully matched to the 12 of the 18 participants from part one of the study (Table 1). During the perturbations, the $1.3 \mathrm{~m} / \mathrm{s}$ group had a greater range in MoS values during Base, Pre and Post1 (Fig. 4). A two-way repeated measures ANOVA revealed a significant effect of group $\left(\mathrm{F}_{[1,22]}=6.409\right.$, 


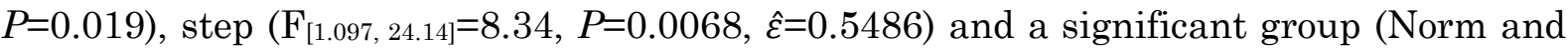
$1.3 \mathrm{~m} / \mathrm{s})$ by step (Base, Pre, Post1) interaction $\left(\mathrm{F}_{[2,44]}=15.4, P<0.0001\right)$ on MoS. Sidak post hoc tests revealed a significant difference between Norm and $1.3 \mathrm{~m} / \mathrm{s}$ groups at Post1 $(P=0.0049)$. While part of the differences found may be due to chance, the current comparison suggests that the stability-normalised walking speed and the normalised perturbation (acceleration to a peak speed $180 \%$ of the walking speed) reduce the interindividual differences in MoS during both unperturbed and perturbed walking, at least with the current protocol. The significant difference found at Post1 between the groups also aligns with the previous studies reporting different responses to perturbations experienced while walking at different speeds (Bhatt et al., 2005, Krasovsky et al., 2014).

\section{Further methodological considerations}

As the MoS - walking speed relationship from $1.0-1.6 \mathrm{~m} / \mathrm{s}$ appeared to be linear in part one of the study (Fig. 1), a simple linear regression was calculated for 1.0-1.6m/s. A significant regression equation was found (Fig. 5). Future research could use this (or similar) as a simple, efficient method for increasing the dynamic similarity in gait stability across participants, by measuring participants walking at a single speed from $1.0-1.6 \mathrm{~m} / \mathrm{s}$ and using this equation to prescribe speeds that would result in similar MoS values. As it is common practice in gait experiments to familiarise participants to the setup and conditions, including some practice walking trials, we would suggest that this may be the
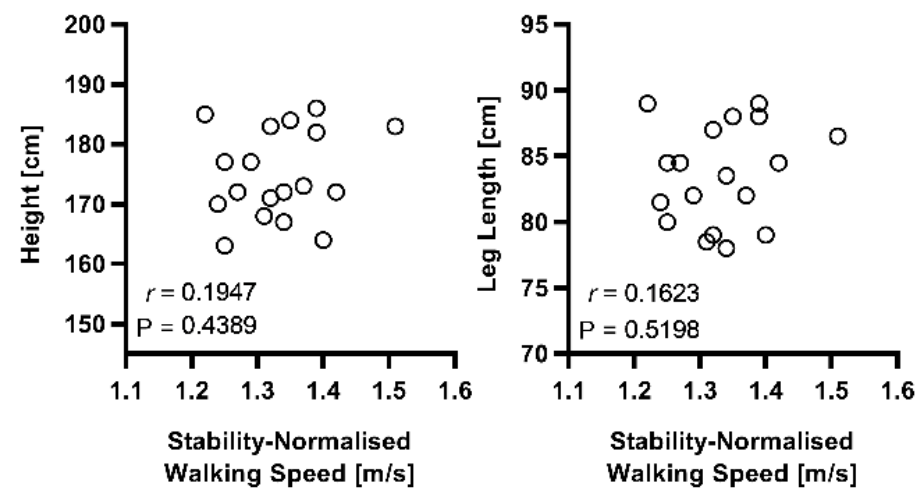

Fig. 3: Pearson correlations between the participants' stability-normalised walking speeds and their height and leg length.

Table 1: Demographic characteristics of the participant groups in part two of the study.

\begin{tabular}{lccccc}
\hline & Sex & Age (y) & Height (cm) & Weight (kg) & Leg Length (cm) \\
\hline $1.3 \mathrm{~m} / \mathrm{s}$ Group & $\begin{array}{c}8 \text { males, } 4 \\
\text { females }\end{array}$ & $25.1 \pm 3.8$ & $178.2 \pm 5.2$ & $72.5 \pm 9.7$ & $84.2 \pm 2.1$ \\
\hline Norm Group & $\begin{array}{c}8 \text { males, } \\
\text { females }\end{array}$ & $24.3 \pm 2.9$ & $178.7 \pm 5.8$ & $79 \pm 15.3$ & $85.5 \pm 2.8$ \\
\hline $\begin{array}{l}\text { Equivalent based on 90\% } \\
\text { Confidence Intervals? }\end{array}$ & - & Yes & Yes & Yes & Yes \\
\hline
\end{tabular}




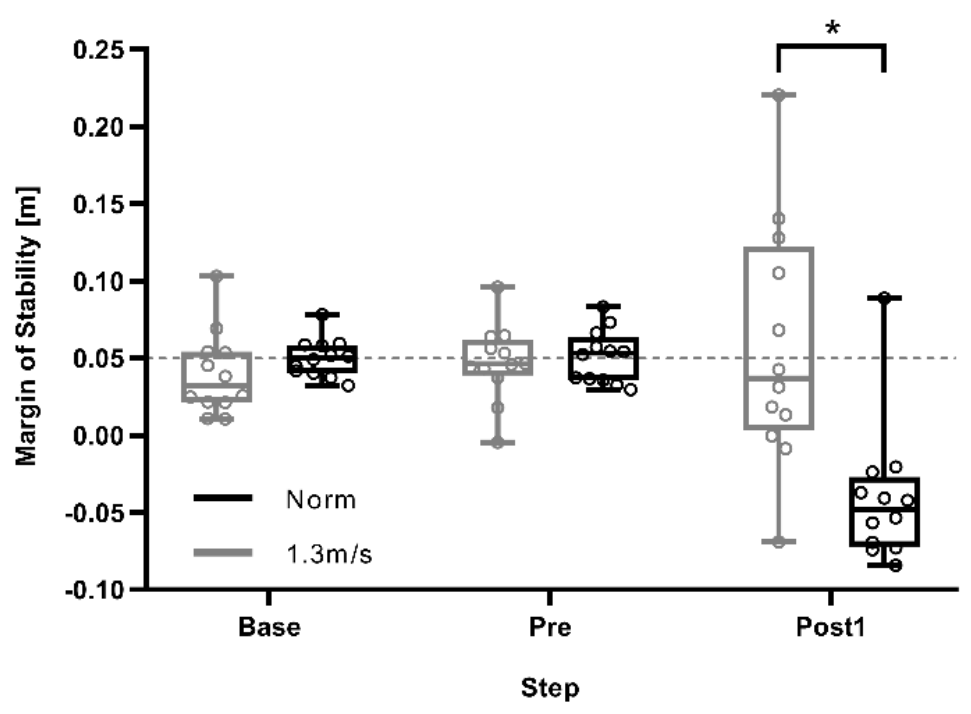

Fig. 4: Margins of stability during unperturbed and perturbed walking of participants walking at their stability-normalised walking speed (Norm) and participants walking at $1.3 \mathrm{~m} / \mathrm{s}$. Base: the mean MoS of the eleventh to second last step before each perturbation; Pre: the final step before each perturbation; Post1: the first recovery step following perturbation. *: Significant difference (Sidak post hoc test: $\mathrm{P}=0.0049$ ).

ideal opportunity to incorporate our method, without having to conduct any additional trials. It is, however, worth highlighting that the current participants were young healthy adults; the walking speed - MoS relationship may be altered in other populations. Future implementations of this method should consider the capacities of the population of interest and the desired or expected impact on gait stability of the perturbations when selecting an MoS value for normalisation.

\section{Limitations}

Individual responses in the MoS to the perturbation varied (Fig. 4), although the variation was lower in the stability-normalised walking speed group. Part of the reasons for this variation could be the result of uncontrolled factors such as individual physiological, biomechanical or psychological differences affecting the individual response at the onset of the perturbation. It could be argued that using a single trial as opposed to averaging multiple trials is less reliable, however, due to the significant and rapid learning effects following even single perturbations of this kind, the responses seen after averaging trials would no longer accurately represent natural responses to unexpected perturbations. In this sense, our approach is ecologically valid, as the variation is representative of daily life responses to truly unexpected gait perturbations. Another potential limitation relates to a validity constraint of the MoS calculation detailed by Hof et al. (2005), in that the pendulum length (distance from the centre of mass to the axis of rotation) should remain constant. This may not always be the case during dynamic walking and perturbed walking tasks if the knee is slightly flexed at foot contact. However, we have not observed large 


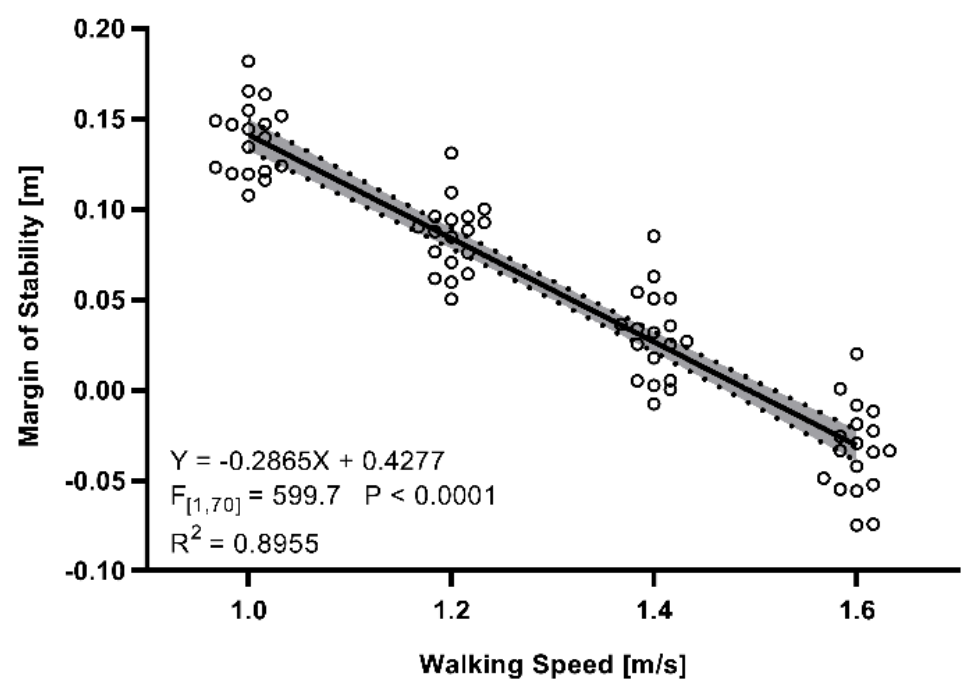

Fig. 5: Margins of stability as a function of walking speed between 1.0 and $1.6 \mathrm{~m} / \mathrm{s}$. The shaded area represents the $95 \%$ confidence intervals of the regression line.

changes in the pendulum length and small changes are not systematic, as within and between individual variability in responses is large. We therefore believe that this is an acceptable limitation of using the model in this context, but one that should be kept in mind when interpreting the results.

\section{Conclusions}

In conclusion, large ranges in MoS were observed and walking speed significantly affected MoS even within these young healthy participants, confirming some issues related to walking speed choice in gait stability research. The current methods reduced betweenparticipant variability in MoS during both unperturbed and perturbed walking, meaning that the method could be beneficial for gait stability studies comparing groups with different locomotor capacities. An equation has been provided that can be used following a single gait trial to increase the dynamic similarity of gait stability between participants.

\section{Acknowledgements}

The authors thank Julia Agmon for assistance with the measurements. 


\section{Part Four: Reactive Gait Stability and Adaptability}

"It does not do to leave a live dragon out of your calculations, if you live near him".

- J.R.R. Tolkien. The Hobbit.

"Kein Operationsplan reicht mit einiger Sicherheit über das erste Zusammentreffen mit der feindlichen Hauptmacht hinaus.”

- Helmuth von Moltke 


\title{
4.1:
}

\section{Retention, savings and interlimb transfer of reactive gait adaptations in humans following unexpected perturbations}

\author{
Christopher McCrum, Kiros Karamanidis, Paul Willems, Wiebren Zijlstra,
} Kenneth Meijer

\section{Published as:}

MCCRUM, C., KARAMANIDIS, K., WILLEMS, P., ZIJLSTRA, W. \& MEIJER, K. 2018. Retention, savings and interlimb transfer of reactive gait adaptations in humans following unexpected perturbations. Commun Biol, 1, 230. doi: 10.1038/s42003-018-0238-9

(C) The Authors 2018. This work is licensed under the Creative Commons Attribution 4.0 International License. http://creativecommons.org/licenses/by/4.0/ 


\begin{abstract}
Reactive locomotor adaptations are crucial for safe mobility, but remain relatively unexplored. Here we assess reactive gait adaptations, and their retention, savings and interlimb transfer. Using new methods to normalise walking speed and perturbation magnitude, we expose eighteen healthy adults to ten unexpected treadmill belt accelerations during walking (the first and last perturbing the right leg, the others perturbing the left leg) on two days, one month apart. Analysis of the margins of stability using kinematic data reveals that humans reactively adapt gait, improving stability and taking fewer recovery steps, and fully retain these adaptations over time. On re-exposure, retention and savings lead to further improvements in stability. Currently, the role of interlimb transfer is unclear. Our findings show that humans utilise retention and savings in reactive gait adaptations to benefit stability, but that interlimb transfer may not be exclusively responsible for improvements following perturbations to the untrained limb.
\end{abstract}




\section{Introduction}

Human locomotion is highly adaptable to environmental change (Choi and Bastian, 2007, Roemmich and Bastian, 2015, Selinger et al., 2015) which ensures effective and safe mobility in daily life. Humans are capable of rapidly adapting gait kinematics in both reactive (McCrum et al., 2014, McCrum et al., 2017b, Epro et al., 2018a) and predictive (Roemmich and Bastian, 2015, McCrum et al., 2016a) manners and such adaptation can be retained over time (Bhatt et al., 2012, Pai et al., 2014b, Roemmich and Bastian, 2015, Epro et al., 2018b) and transferred between different locomotor tasks and environmental conditions (Bieryla et al., 2007, Reisman et al., 2009, Torres-Oviedo and Bastian, 2010, Parijat and Lockhart, 2012, Yang et al., 2013, Long et al., 2015). In particular, reactive gait adaptations are of great interest for falls prevention research and are the focus of perturbation-based balance training for populations at an increased risk of falls (Pai and Bhatt, 2007, Mansfield et al., 2010, Pai et al., 2014a, Mansfield et al., 2015b, Gerards et al., 2017, McCrum et al., 2017b, Epro et al., 2018b). However, the retention (a preservation over time of adaptations made previously), savings (faster re-adaptation on re-exposure to a perturbation) and transfer (changes in an untrained limb or task reflecting, at least to some extent, the changes seen in the trained limb or task) of reactive gait adaptations are not yet well understood, despite their importance for falls prevention interventions. Reactive gait adaptability implies that the neuromuscular system can alter its behaviour in a feedback-driven manner, meaning that modulation of spinal and sensory reflex pathways may be occurring. While spinal plasticity, in general, is well supported in humans (Wolpaw, 2010, Wolpaw, 2012) and has been demonstrated during walking in animals (Forssberg et al., 1975, Forssberg et al., 1977, Frigon and Rossignol, 2008, Zhong et al., 2012, Dambreville et al., 2015), little is known about whether specifically reactive gait adaptations in humans are amenable to savings and transfer between the lower limbs. These adaptation qualities may be quite different from those occurring during predictive gait adaptation, which involve supraspinal processes (Morton and Bastian, 2006, Vasudevan et al., 2014).

Two examples of unilateral lower limb reflexes that are purported to support safe locomotion are limb withdrawal reflexes during the stance phase (Spaich et al., 2004) and stumble correction reflexes during the swing phase (Haridas et al., 2008) of gait (i.e. quick removal of the limb if an unsafe object is stepped upon during the stance phase or contacted during the swing phase). It has also been suggested that interlimb reflexes (as evidenced by responses in the contralateral limb following perturbation of the ipsilateral limb) support gait stability control (Stubbs et al., 2011, Hoogkamer et al., 2012, Stevenson et al., 2013, Gervasio et al., 2015, Stevenson et al., 2015). Note that these studies have used a variety of methods to perturb the lower limbs, including direct nerve stimulation, single joint perturbations and perturbations that have a whole-body effect, which may result in very different responses and adaptations. Such stumbling and interlimb reflexes have also been observed (Yang et al., 1998, Lam et al., 2003) and have been shown to adapt (Lam et al., 2003, Pang et al., 2003) following repeated simulated trip perturbations in infants prior to independent walking, indicating that adaptation of these reflexes can occur in a feedback-driven manner, without substantial supraspinal influence. That is not to say that supraspinal structures do not influence balance control in human adults, as 
there is ample evidence to the contrary (Jahn et al., 2008, Stevenson et al., 2013, Bruijn et al., 2015, Martinez et al., 2016, Peterson and Horak, 2016, Frigon, 2017, Mierau et al., 2017, Wittenberg et al., 2017, Mrachacz-Kersting et al., 2018), but our knowledge of the supraspinal influence on reactive gait stability control during unexpected mechanical perturbations, specifically, is currently limited .

Despite evidence of feedback-driven adaptation in these reflexes during specific stimulation or joint level perturbations and in gait stability control following whole body mechanical perturbations, whether or not this translates to the retention, savings and interlimb transfer of adaptations in reactive gait stability following mechanical, whole body perturbations such as slips and trips has not, to our knowledge been addressed in the literature. There is evidence to suggest that humans can at least partly retain reactive adaptations in gait stability over different time periods of months to years (Bhatt et al., 2012, Pai et al., 2014b, Liu et al., 2017, Epro et al., 2018b). However, no study has examined savings and the interlimb transfer of reactive gait adaptations to standardised, controlled whole body (mechanical) perturbations. As these processes are of both fundamental and clinical relevance for understanding human locomotor control, further research into these processes is warranted.

Here we assess the reactive adaptation of gait in response to unexpected, repeated gait perturbations in young healthy adults, how this adaptation is retained after one month, and if savings and interlimb transfer of these adaptations can be observed. To achieve this in as controlled and as precise a manner as possible, we use new methods to decrease inter-individual differences in gait stability via a normalisation of walking speed based on gait stability and by perturbing gait with a treadmill belt acceleration standardised to the stability-normalised walking speed (McCrum et al., 2019b) (preprint version). Thereby, we account for the effects of walking speed on gait stability control and measurement that we have previously outlined (McCrum et al., 2016b, McCrum et al., 2017b, McCrum et al., 2019b). The margin of stability measure (Hof et al., 2005) was used to assess gait stability as it is a valid measure of the mechanical stability of the body configuration during large balance perturbations (Arampatzis et al., 2008, Bruijn et al., 2013). It was hypothesised that healthy young adults would demonstrate reactive adaptation of gait following repeated gait perturbations, that these adaptations would be partly retained one month later, that evidence of savings in both the acute response to a single perturbation and in the recovery behaviour over multiple perturbations would be found, and that the adaptation to repeated perturbations to one lower limb would transfer and benefit gait stability following perturbations to the contralateral lower limb, as the recovery requires a bipedal response that may be generalizable.

The results of the current study show that young healthy adults can adapt their gait in a reactive, feedback driven manner and reduce the number of steps required to recover balance following unexpected perturbations to gait and retain these adaptations over a one-month period. Combined retention and savings led to further improvements in reactive stability control during the second measurement one month later. Evidence of interlimb transfer of reactive gait adaptations was inconclusive. Our findings suggest that young healthy adults utilise retention and savings in reactive gait adaptations to benefit 
stability, but that improvements in stability following perturbations to the untrained limb may not be exclusively due to interlimb transfer of adaptations.

\section{Results}

\section{Study overview}

In order to test our hypotheses, 18 healthy young adult participants were subjected to 10 unilateral treadmill belt accelerations during walking on one day, as shown in Figure 1 (see methods section for details). The participants returned approximately one month later (28.4 \pm 3.4 days) and repeated the perturbation protocol, although the participants were only aware that they would complete a "walking balance challenge" and were told that it could be different on the second day. The gait perturbation protocol was conducted at a stability-normalised walking speed based on trials of unperturbed walking at various speeds for each individual, to ensure that all participants were walking at comparable stability levels (McCrum et al., 2019b). The stability-normalised walking speeds ranged from $1.22 \mathrm{~m} \cdot \mathrm{s}^{-1}$ to $1.51 \mathrm{~m} \cdot \mathrm{s}^{-1}$ with a mean \pm SD of $1.33 \pm 0.07 \mathrm{~m} \cdot \mathrm{s}^{-1}$. In order to quantify stability, we determined the anteroposterior MoS at the moment of foot touchdown as defined by Hof et al. (2005), adapted for a reduced kinematic model based on Süptitz et al. (2013). Representative data from one participant during a perturbation and during fast walking is shown in Figure 2, alongside schematic representations of the body configuration at specific time points, to illustrate how the components of the MoS are affected by different walking conditions.

In the following results, data are presented as median and $95 \%$ confidence intervals unless otherwise stated. Day 1 values are represented by filled symbols, Day 2 values by empty symbols. Perturbations to the right leg are represented by squares and perturbations to the left leg by circles. Perturbations of the same number (i.e. Pert1 $1_{\mathrm{R}}$ ) are represented by the same colours. The data used to create each figure can be found in "Supplementary Data".

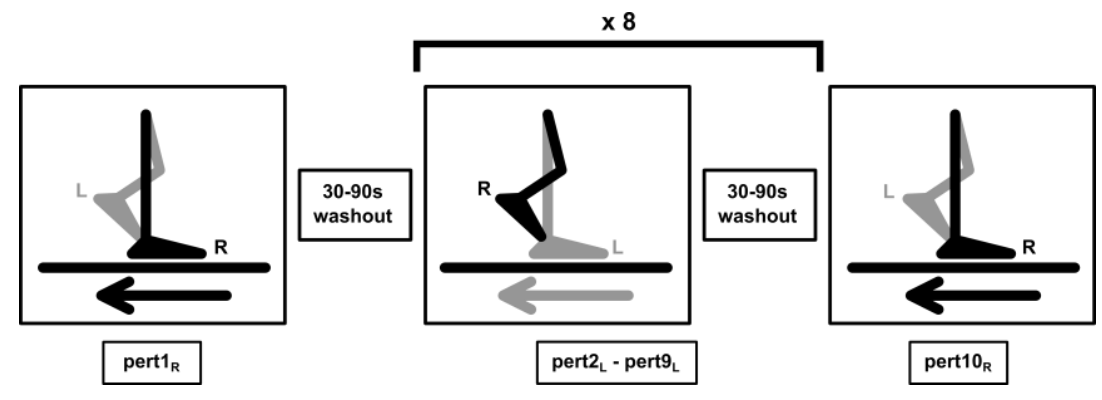

Fig. 1: Gait perturbation protocol. The right leg (R) was perturbed by the treadmill belt acceleration first (pert $1_{R}$ ), followed by eight perturbations (pert $2_{L}-$ pert $9_{L}$ ) to the left leg (L), and the final perturbation (pert10 ${ }_{R}$ ) was again applied to the right leg $(\mathrm{R}) .30-90$ seconds time of unperturbed walking occurred between each perturbation. The perturbation consisted of a $3 \mathrm{~m} / \mathrm{s}^{2}$ acceleration of the treadmill belt to $180 \%$ of the stability-normalised walking speed, triggered automatically when the vertical projection of the hallux marker of the to-beperturbed limb became anterior to the hallux marker of the stance limb in the sagittal plane. The perturbation was designed to cause a forward rotation and acceleration of the upper body, relative to the lower body, leading to a forward loss of dynamic stability. 
a

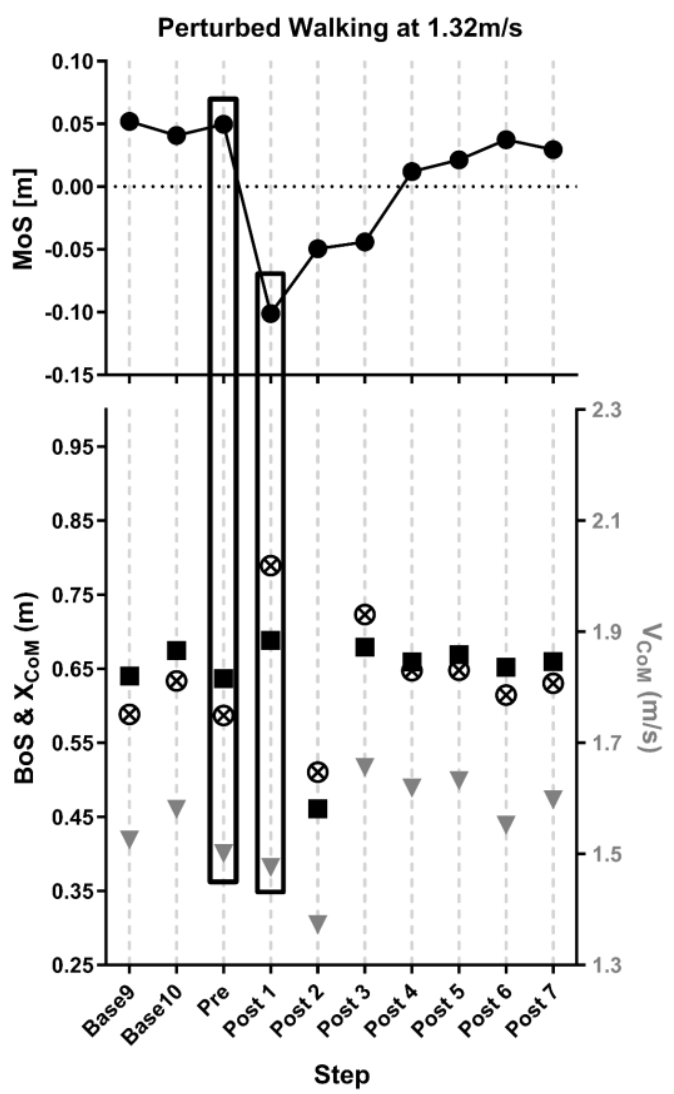

4.1

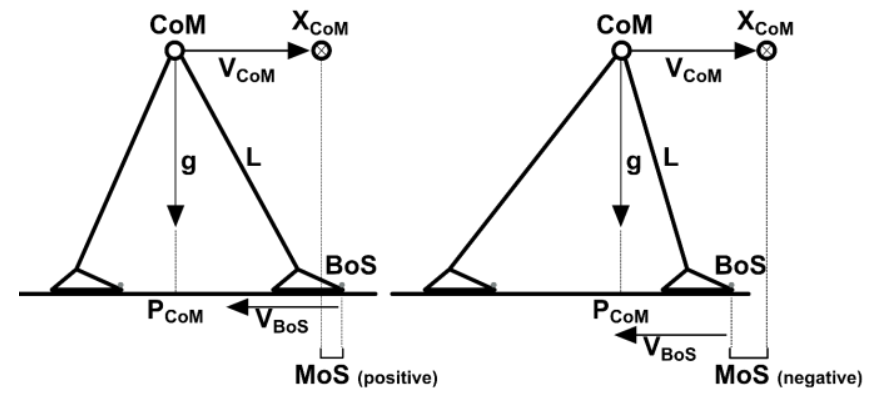

Pre b
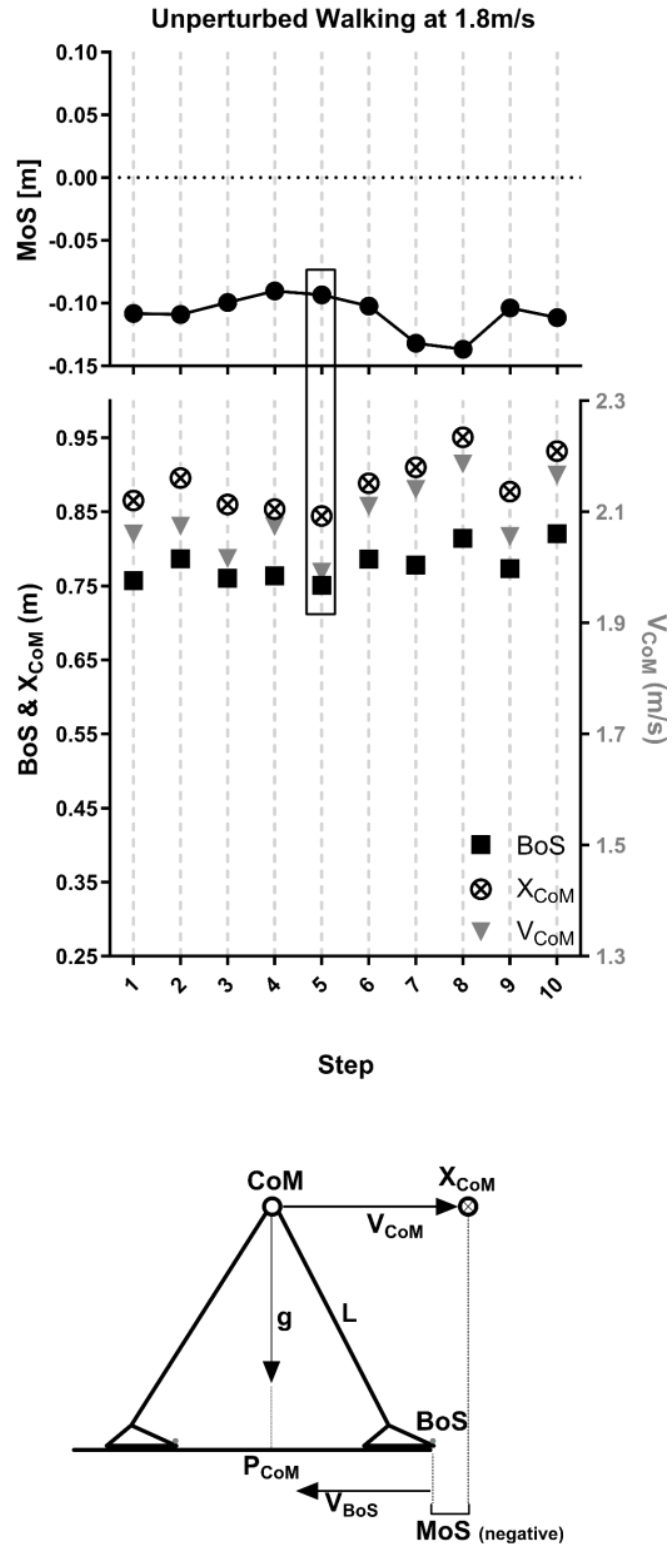

Step 5

Fig. 2: The margin of stability (MoS) components during perturbed and unperturbed walking. Representative data from one individual participant during a perturbation (a) and during fast unperturbed walking (b), as well as schematic representations of the body configurations and margin of stability components at foot touchdown of the step before the perturbation (Pre), the first step post-perturbation (Post 1) and for one step during fast unperturbed walking that elicited a similar margin of stability to Post 1 (Step 5). The anteroposterior MoS were calculated for the moment of foot touchdown as the anteroposterior difference between the base of support (BoS; anteroposterior distance between the hallux markers) and the extrapolated centre of mass $\left(\mathrm{X}_{\mathrm{Com}}\right)$ as defined by Hof et al. (2005), adapted for the reduced kinematic model(Süptitz et al., 2013). The BoS, $\mathrm{X}_{\mathrm{CoM}}$ and MoS are indicated on the left $\mathrm{y}$ axes, and the velocity of the centre of mass $\left(\mathrm{V}_{\mathrm{CoM}}\right)$ on the right $\mathrm{y}$ axes. The $\mathrm{BoS}$ and $\mathrm{X}_{\mathrm{Com}}$ positions are relative to the posterior hallux marker position in the anteroposterior direction. Note that while Step 5 displays a comparable MoS value to Post 1, the absolute and relative positions of the components of the MoS are not all the same, indicating that the change in belt velocity during the perturbation is not the sole reason for the change in MoS. 


\section{Reactive Gait Adaptations to Repeated Perturbations}

The two-way repeated measures ANOVAs revealed significant perturbation number and step effects and significant perturbation number by step interactions on the MoS for Day $1 \quad\left(\mathrm{~F}_{[3,51]}=7.117, P=0.0004 ; \mathrm{F}_{[9,},{ }_{153]}=39.05, P<0.0001 ;\right.$ and $\mathrm{F}_{[27,459]}=2.788, P<0.0001$, respectively) and Day $2\left(\mathrm{~F}_{[3,51]}=14.69, P<0.0001 ; \mathrm{F}_{[9,153]}=49.11, P<0.0001 ;\right.$ and $\mathrm{F}_{[27}$, 459] $=5.943, P<0.0001$, respectively). Tukey's multiple comparisons tests revealed that MoS during Base and Pre were not significantly affected by perturbation number $(0.30<P<0.99$; see Supplementary Table 1). Regarding the adaptation of gait on Day 1, the participants were able to return to MoS Base values after five and four post-perturbation steps for Pert2L and Pert9L, respectively, indicated by MoS values significantly different to Base for Post1-5 and Post1-4, respectively (Fig. 3; for detailed multiple comparisons results, see Supplementary Table 2). On Day 2, further adaptation across the left leg perturbations was seen as Pert9L required only two recovery steps for participants to regain stability, compared with four steps during Pert2L, indicated by MoS values significantly different to Base for Post1-2 and Post1-4, respectively (Fig. 3; Supplementary Table 3).

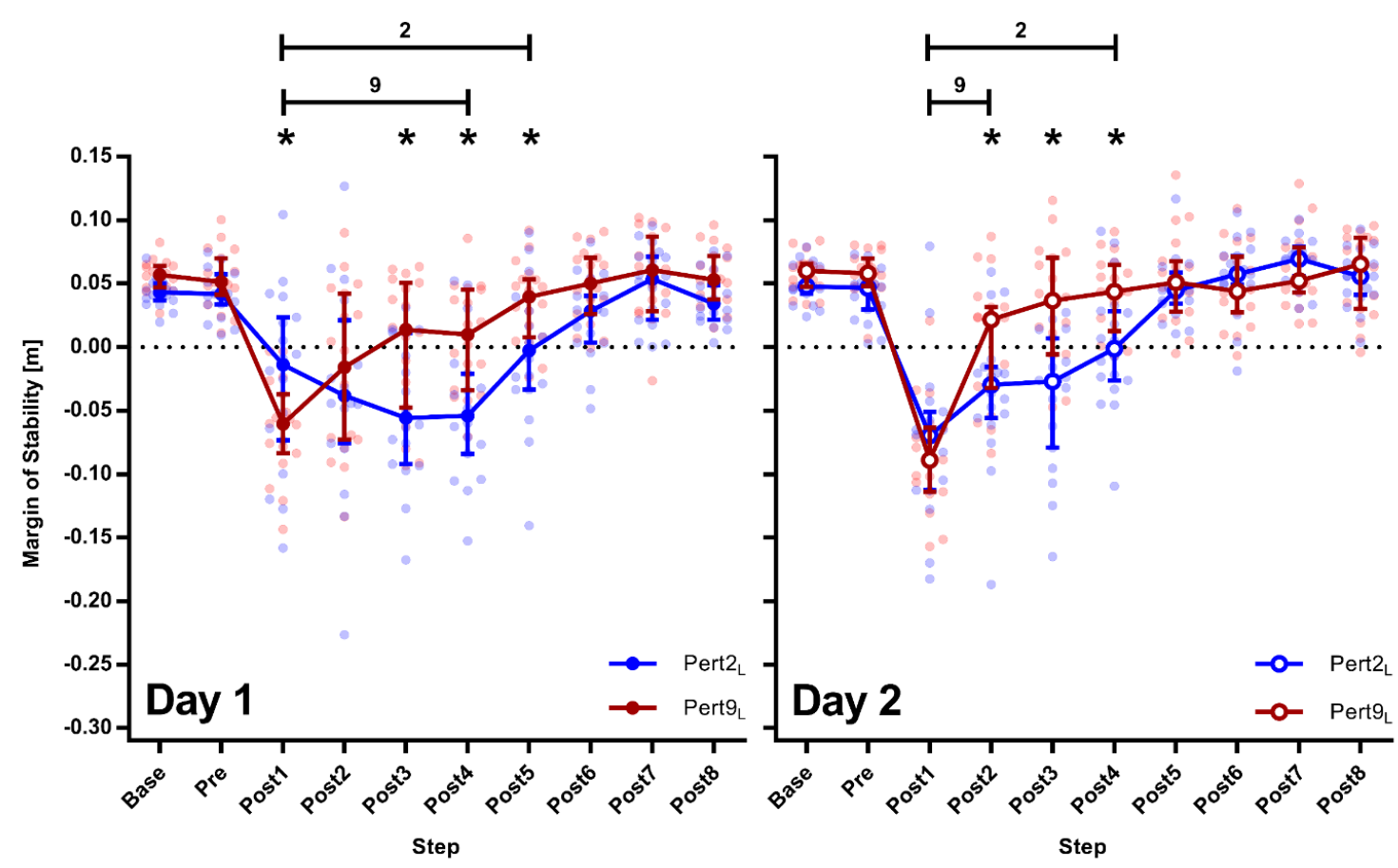

Fig. 3: Reactive adaptations in gait during repeated perturbations. Median and 95\% confidence intervals of the margins of stability during the second and ninth perturbations (the first and final perturbation of the left leg; Pert $2_{\mathrm{L}}$, and Pert $9_{\mathrm{L}}$, respectively) during unperturbed walking prior to each perturbation (Base), the final step prior to each perturbation (Pre) and the first eight recovery steps following the perturbations (Post1-8) on Day 1 (left panel) and Day 2 (right panel) of the measurements. *: Significant difference between Pert $2_{\mathrm{L}}$ and Pert9 $\mathrm{L}_{\mathrm{L}}(\mathrm{P}<0.05)$. Lines with 2 and 9: All steps under the line were significantly different to Base for Pert $2_{\mathrm{L}}$, and Pert $9_{\mathrm{L}}$, respectively $(\mathrm{P}<0.05)$. 


\section{Retention of Reactive Adaptations in Gait}

Regarding retention of the Day 1 adaptations to perturbations of the left leg after one month, Pert2L on Day 2 resulted in participants requiring the same number of recovery steps (four) before returning to MoS Base as during Pert9L on Day 1 (Fig. 4 and Supplementary Tables 2 and 3). A direct comparison of Day 1 Pert9L vs. Day 2 Pert2L revealed a significant perturbation number by step interaction $\left(\mathrm{F}_{[9,153]}=2.696, P=0.0061\right)$ and the post hoc comparisons revealed significant differences for Post3 only $(P=0.0002$; Fig. 4).

\section{Interlimb Transfer and Savings of Gait Adaptations}

The adaptation to perturbations applied to the left leg did not appear to transfer to stability recovery following perturbations to the right leg on Day 1, as no significant differences were found between Pert $1_{R}$ and Pert10 $R$ for any step (Fig. 5; also see Supplementary Table 4) and the number of steps needed post-perturbation to return to MoS Base was the same during Pert $1_{R}$ and Pert10R (Fig. 5; also see Supplementary Table 2). However, Pert1 $1_{\mathrm{R}}$ on Day 2 required one step less in order to recover to MoS Base,

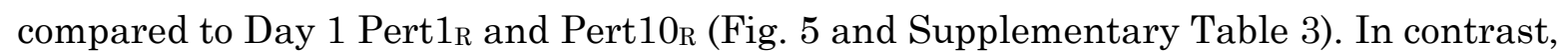

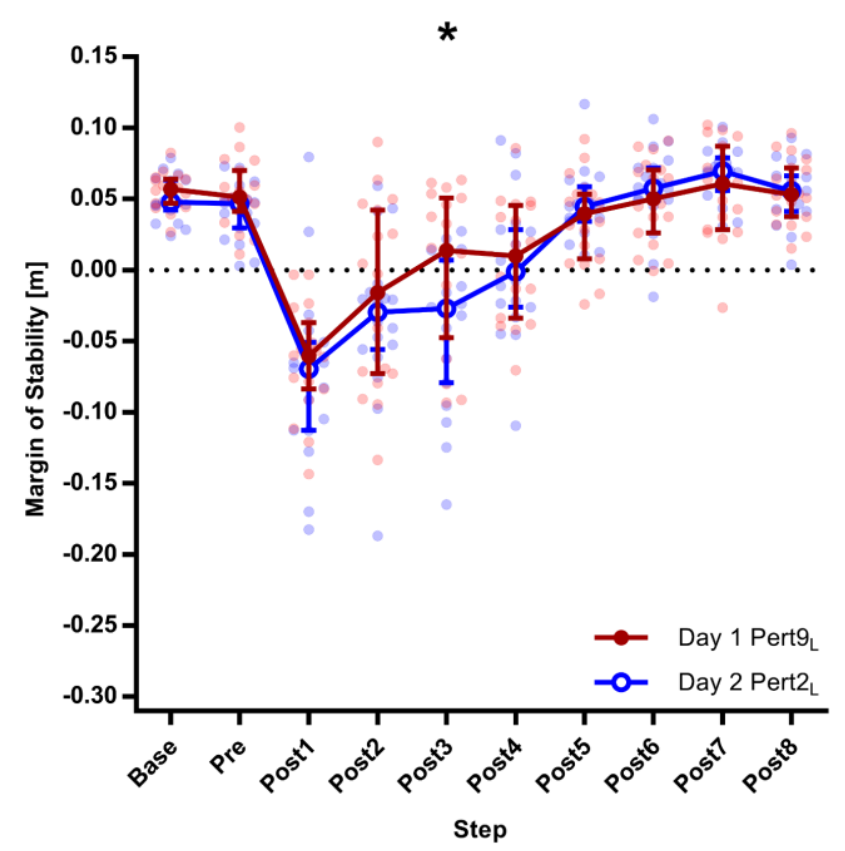

Fig. 4: Retention of reactive adaptations in gait. Median and 95\% confidence intervals of the margins of stability during the ninth perturbation on Day 1 and the second perturbation on Day 2, representing the final and first perturbations to the left leg on Day 1 and Day 2, respectively (Day 1 Pert9 $9_{\mathrm{L}}$ and Day 2 Pert $2_{\mathrm{L}}$, respectively) during unperturbed walking prior to each perturbation (Base), the final step prior to each perturbation (Pre) and the first eight recovery steps following the perturbations (Post1-8). *: Significant difference at the indicated step between Day 1 Pert $_{\mathrm{L}}$ and Day 2 Pert $2_{\mathrm{L}}(\mathrm{P}=0.0002)$. 
the adaptation to perturbations applied to the left leg on Day 2 did appear to transfer and benefit stability recovery following perturbations to the right leg, as significant differences were found between Pert1R and Pert10R for Post2-5 (Fig. 5; also see Supplementary Table $5)$, although the number of steps needed post-perturbation to return to MoS Base was the same (four) during Pert1 $1_{\mathrm{R}}$ and Pert10 (Fig. 5; also see Supplementary Table 3). To further investigate the results regarding interlimb transfer of reactive adaptations in gait, posthoc analyses were conducted (see below).

The presence of savings was unclear, due to the almost complete retention of adaptations on Day 2 (Pert2_ Day 2 vs. Pert9 L Day 1; Figs. 3 and 4; Supplementary Tables 2 and 3). Post-hoc analyses were conducted to further investigate savings (see below).

\section{Post-Hoc Analyses of Savings and Interlimb Transfer}

As full retention was seen in the number of steps to recover to MoS Base in Day 2 Pert2L, compared to Day 1 Pert9L, it was unclear from the pre-planned analysis if savings in the recovery response were present. To investigate the possible presence of savings in the acute recovery response, Pert2L from each day was analysed in a two-way repeated measures ANOVA with day and step as factors, with Bonferroni's test for multiple
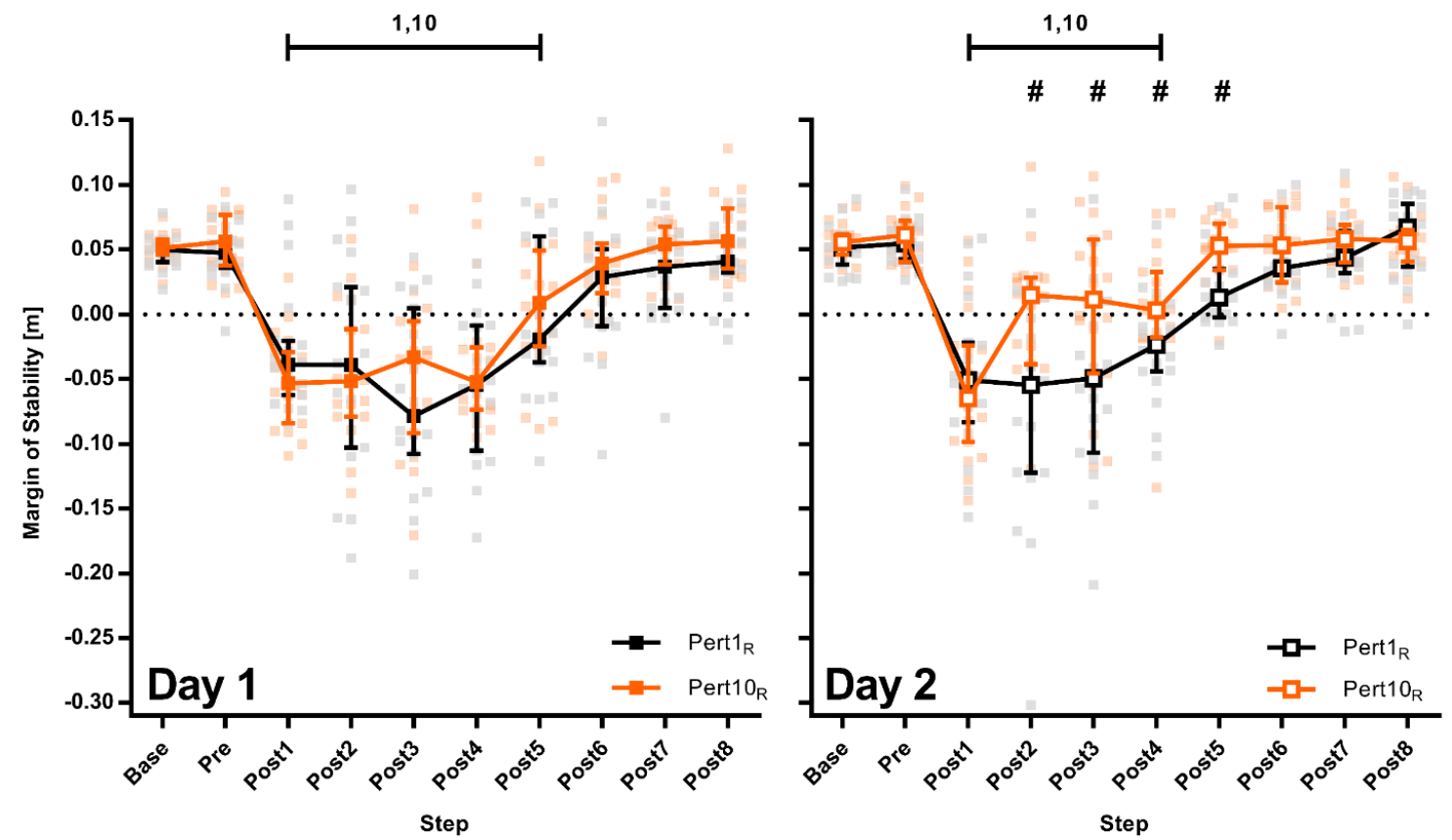

Fig. 5: Interlimb transfer of reactive adaptations in gait. Median and 95\% confidence intervals of the margins of stability during the first and tenth perturbations (the first and final perturbation of the right leg; Pert $1_{R}$, and Pert $10_{R}$, respectively) during unperturbed walking prior to each perturbation (Base), the final step prior to each perturbation (Pre) and the first eight recovery steps following the perturbations (Post1-8) on Day 1 (left panel) and Day 2 (right panel) of the measurements. \#: Significant difference between Pert $1_{R}$ and Pert $10_{R}$ $(\mathrm{P}<0.05)$. Lines with 1 and 10: All steps under the line were significantly different to Base for Pert $1_{R}$ and Pert10 $0_{R}$, respectively $(\mathrm{P}<0.05)$. 
comparisons. This analysis revealed evidence of savings, as the rate of recovery to MoS Base was significantly faster (savings), with significant post hoc differences between Pert2L on Days 1 and 2 at Post 4 and Post5 (Fig. 6A and Supplementary Table 6). To further investigate savings in the overall recovery response, two way repeated measures ANOVAs with step and perturbation number as factors with Bonferroni's test for multiple comparisons were conducted for all perturbations on Day 1 and Day 2, and revealed that the number of steps required to reach MoS baseline after the perturbations plateaued at four steps from the third perturbation onwards on Day 1, while on Day 2, as little as two steps where required by the sixth perturbation (Fig. 6B and Supplementary Table 7). The numbers of steps to return to MoS baseline are summarised in Fig. 6B, and the full results of these ANOVAs can be found in Supplementary Table 7.

The pre-planned analysis appeared to reveal evidence of interlimb transfer on Day 2 , but not Day 1. In order to explore these findings further, we calculated the number of steps required to reach consistently positive MoS values following Pert1R, Pert2L, Pert9L and Pert10R on each day, for each individual. A two-way repeated measures ANOVA with day and perturbation number as factors with Bonferroni's test for multiple comparisons revealed significant day $\left(\mathrm{F}_{[1,17]}=8.951, P=0.0082\right)$ and perturbation number $\left(\mathrm{F}_{[3,51]}=15.79\right.$,
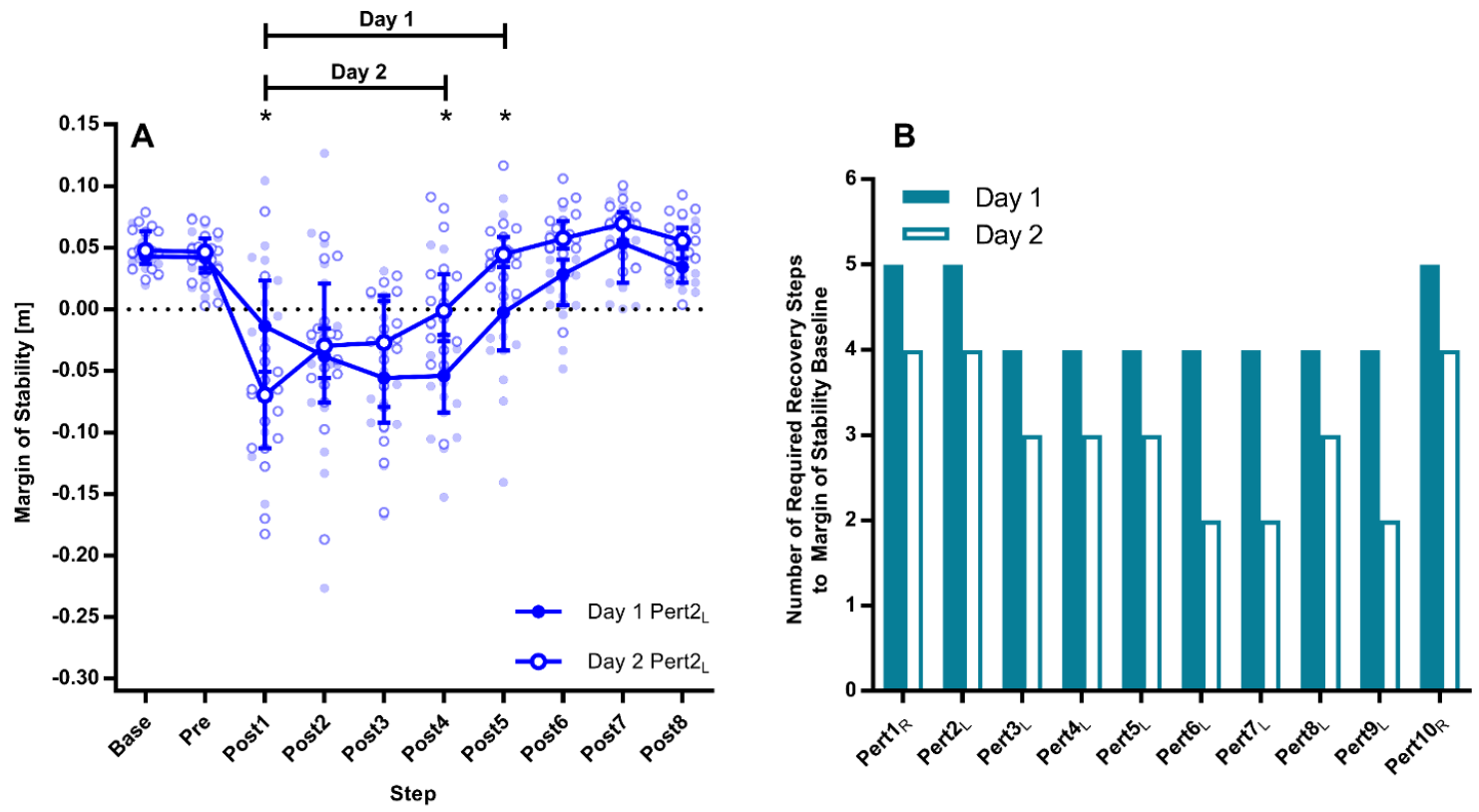

Fig. 6: Savings in reactive adaptations in gait. A: Median and 95\% confidence intervals of the margins of stability during the first perturbation to the left leg on Day 1 and Day 2 (Day 1 Pert2 $\mathrm{L}$ and Day 2 Pert2 ${ }_{\mathrm{L}}$, respectively) during unperturbed walking prior to each perturbation (Base), the final step prior to each perturbation (Pre) and the first eight recovery steps following the perturbations (Post1-8). *: Significant difference between Day 1 and Day 2 $(\mathrm{P}<0.05)$. Lines with Day 1 and Day 2: All steps under the line were significantly different to Base for Day 1 and Day 2, respectively $(\mathrm{P}<0.05)$. B: The number of steps required during each of the ten perturbations on Day 1 and Day 2 to return to baseline margins of stability as assessed by two-way repeated measures ANOVAs with Bonferroni's multiple comparisons tests. 
$P<0.0001$ ) effects on the number of steps to reach positive MoS (Fig. 7). Regarding interlimb transfer, Pert10R on Day 2 required significantly fewer steps for participants to reach positive MoS values compared to Pert1R on Day $2(P=0.0016)$ and Pert10R on Day 1 $(P=0.0016$; Fig. 7).

To determine if the apparent interlimb transfer of adaptations in stability on Day 2 (see Figs. 5 and 7) were purely due to transfer, or partly due to a practice effect of the right leg, a two-way repeated measures ANOVA with step and perturbation number as factors with Bonferroni's test for multiple comparisons were conducted for the fourth perturbation to each leg: Day 1 Pert5L and Day 2 Pert10R, respectively. No significant effect of perturbation number was found. However, during Day 1 Pert5L, three steps were needed to return to baseline MoS, whereas during Day 2 Pert10R, five steps were needed (Fig. 8).

\section{Discussion}

In this study, we assessed the reactive adaptation of gait in response to repeated gait perturbations in young healthy adults, how this adaptation was retained after one month (28.4 \pm 3.4 days), and if savings and interlimb transfer of these adaptations could be observed. We hypothesised that healthy young adults would demonstrate reactive adaptation of gait following repeated gait perturbations, that these adaptations would be partly retained one month later, that evidence of savings in both the acute response to a single perturbation and in the general recovery behaviour over multiple perturbations would be found, and that evidence of interlimb transfer of adaptations in stability would
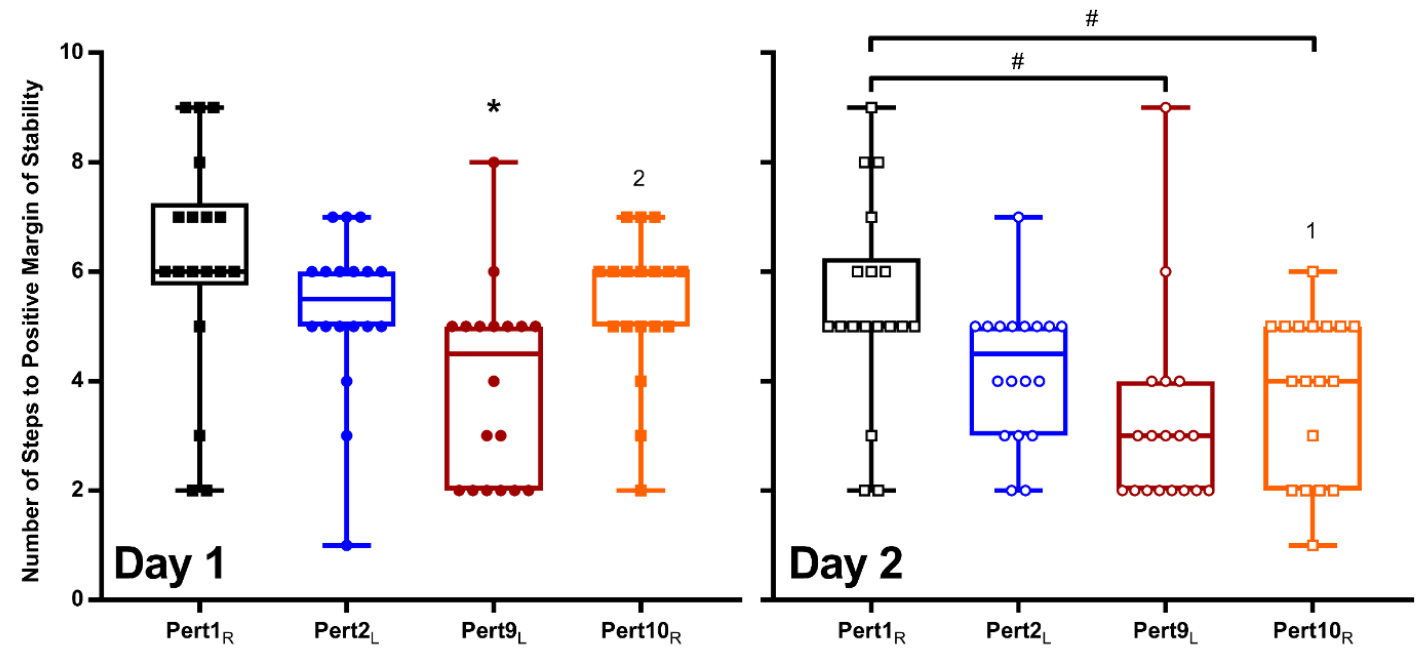

Fig. 7: Number of steps to positive margins of stability. Box plots and individual data points of the number of steps required to reach positive margins of stability following the first and final perturbation to each lower limb (Pert $1_{R}$, Pert $2_{L}$, Pert $9_{L}$ and Pert $\left.10_{R}\right)$ on each day for each participant. *: Significantly different to all other perturbations on that day $(\mathrm{P}<0.05)$. \#: Significant difference between the indicated perturbations $(\mathrm{P}<0.01) .1$ or 2 : Significant difference to that same perturbation number on Day 1 or Day 2 , respectively $(\mathrm{P}<0.01)$. 


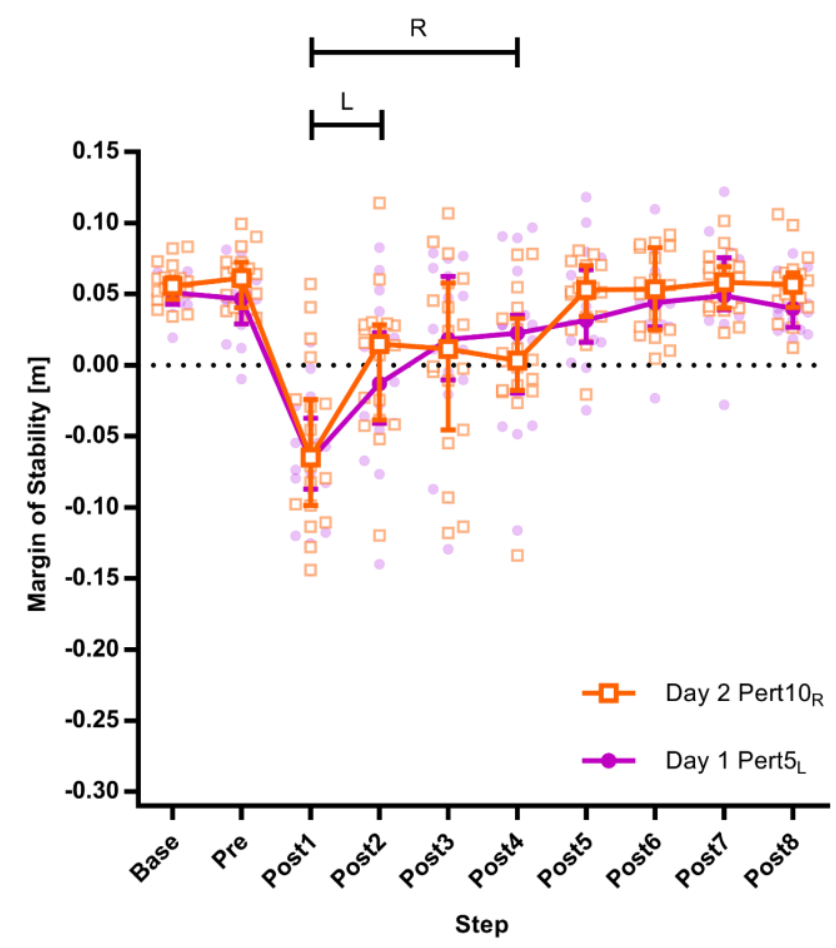

Fig. 8: Comparison of the reactive responses to the fourth perturbation of each limb. Median and $95 \%$ confidence intervals of the margins of stability during the fourth perturbation to each leg (Day 1 Pert $5_{\mathrm{L}}$ and Day 2 Pert $10_{\mathrm{R}}$, respectively) during unperturbed walking prior to each perturbation (Base), the final step prior to each perturbation (Pre) and the first eight recovery steps following the perturbations (Post1-8). Lines with R and L: All steps under the line were significantly different to Base for the right and left limb, respectively $(\mathrm{P}<0.05)$.

be found. The first and second hypotheses regarding adaptation and retention were confirmed, as significant improvements in MoS and number of steps to MoS Base during the perturbations to the left leg on the first day were observed, and these improvements were (almost completely) retained during the first perturbation to the left leg on the second measurement day (post one month), confirming previous work demonstrating adaptation and retention in reactive gait stability (Bhatt et al., 2012, McCrum et al., 2014, Pai et al., 2014b, Epro et al., 2018a, Epro et al., 2018b). The third hypothesis regarding savings was confirmed in post-hoc analyses, as the pre-planned analysis could not confirm this due to the unexpected extent of the retention observed. Finally, our hypothesis that evidence of interlimb transfer of adaptations in gait stability would be observed was not conclusively supported nor refuted. No clear difference between stability during the two right leg perturbations on Day 1 were seen, but improvements in the recovery during the right leg perturbations on Day 2 were found. However, we could not conclusively determine if the gait adaptations observed during the right leg perturbations on Day 2 were strictly due to interlimb transfer or to independent or combined effects of interlimb transfer, perturbation repetition, and task awareness. 
The current study deals with reactive (feedback-driven) adaptations in gait, and how these are retained, saved and transferred. We must first confirm that these adaptations were indeed predominantly reactive, and that they were not significantly influenced by predictive (feedforward) adaptations in gait. Predictive adaptations in gait did not occur, at least not in a way that influenced the MoS, as Base and Pre were not significantly affected by perturbation number (Supplementary Table 1). This may be, in part due to our perturbation paradigm, as no visual cues occurred for these treadmill delivered perturbations, as may be the case in overground situations (McCrum et al., $2017 \mathrm{~b})$ and the timing between perturbations was variable and unpredictable. Taking these considerations together, we assume that the results represent predominately reactive adaptations in gait, and were not due to predictive adjustments. Previous studies have also found independent adaptations in reactive stability control (Pavol et al., 2004, Lam et al., 2006, Pai et al., 2014b, Welch and Ting, 2014, Patel and Bhatt, 2015). However, the current study design does not allow us to determine how much of the adaptations observed in gait stability control can be attributed to direct modulation of spinal reflexes as seen in response to simulated trip perturbations in infants prior to independent walking (Lam et al., 2003, Pang et al., 2003). In the current setup, a number of these reflexes may have been adapted, as treadmill belt accelerations have been shown to induce stretch reflexes of the plantar flexor muscles (Sloot et al., 2015) and interlimb reflexes (Berger et al., 1984). Such reflexes can also be modulated based on the walking environment and the potential threat to gait stability (Schneider and Capaday, 2003, Haridas et al., 2008), meaning that once the participants in the current study had experienced a perturbation, certain reflexes may have been modulated to elicit faster or greater responses, which could partly explain the improved gait stability and further adaptation observed on Day 2. Given this evidence, it seems reasonable to assume that the adaptation of gait observed in the current study could be, at least in part, due to feedback-driven adaptations of spinal reflexes that are important for gait stability.

An interesting outcome on Day 1 was that some participants demonstrated an increase, rather than a decrease in stability at Post1 during Pert2L (see Fig. 9 for the individual values). With repetition of the perturbations, participants adapted towards a decrease in stability (Pert9L, Fig. 9), which was maintained on Day 2. At first glance, this change does not appear logical; why would participants decrease their stability with practice? On closer inspection of the data and of the video recordings, it appears that some individuals created a large increase in the base of support with a large anterior step, to prevent a forward loss of balance, resulting in an increase in the MoS. However, as the treadmill was moving at a fixed speed, participants then had to "catch up" with the treadmill belt. This strategy appears to initially prioritise stability control and neglect the secondary task of continuing walking.

Our results demonstrated that young healthy adults are capable of almost fully retaining reactive adaptations in gait over a period of one month. Previous work has repeatedly demonstrated partial retention of reactive gait adaptations in healthy adults (Bhatt et al., 2012, Pai et al., 2014b, Liu et al., 2017, Epro et al., 2018b). The reasons why our participants demonstrate almost full retention are unclear, but could be related to the nature of the perturbation, the normalisation procedure or awareness of the task. What 


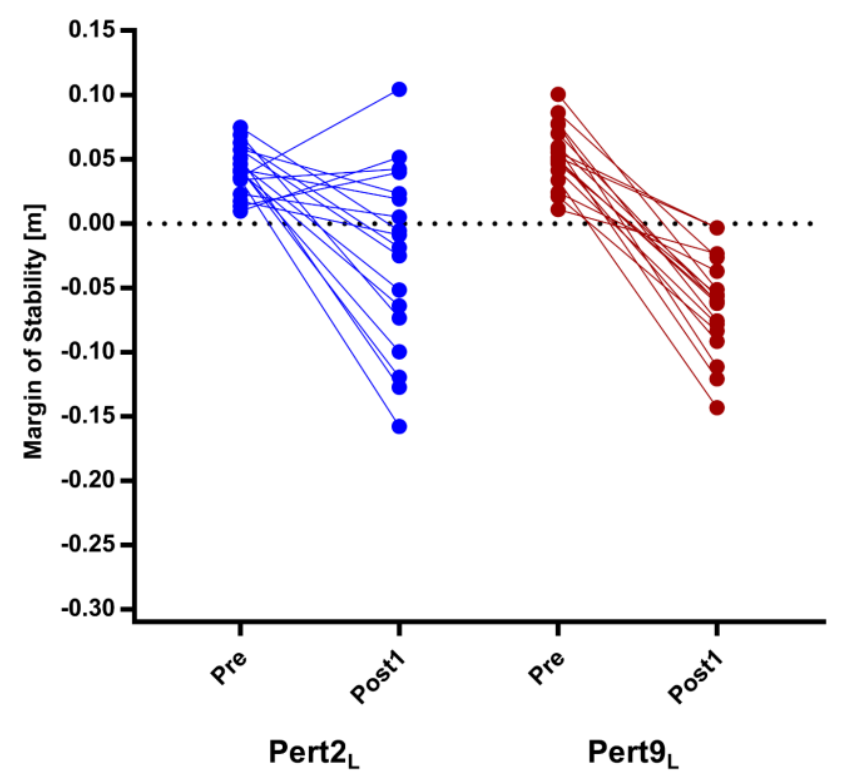

we can conclude from these results is that young adults do not necessarily need frequent or consecutive exposure to unexpected gait perturbations to improve their reactive gait stability. This finding aligns with one recent study that showed that older adults need only one overground slip perturbation to trigger beneficial, long-lasting adaptations in stability control (Liu et al., 2017).

In contrast to our hypothesis, no interlimb transfer of adaptations in reactive gait stability appeared to occur on Day 1. This result was surprising for two reasons. Firstly, while the perturbations were applied to one leg specifically, multiple recovery steps are needed following such perturbations to control balance (i.e. both legs are necessary for recovery), as well as upper body control and counter rotations (Hof, 2007). Therefore, we suspected that the response during and immediately following each perturbation (i.e. the first recovery step) may be limb specific, due to different requirements for braking and propulsion similar to previous suggestions for slip recovery (Bhatt and Pai, 2008), but that the following alterations in gait would be generalizable and could consequently benefit stability control. Secondly, as opposed to most previous studies on interlimb transfer in gait (of which one has shown that adaptations transfer across limbs (van Hedel et al., 2002) while others have not (Prokop et al., 1995, Choi and Bastian, 2007, Houldin et al., 2012)), our paradigm required reactive adaptation to repeated, unexpected perturbations, not feedforward correction of errors during continuous perturbation. Abrupt, as opposed to gradual, gait perturbations provide a substantial amount of error feedback that can aid participants' gait adaptation (van Asseldonk et al., 2011, Hussain and Morton, 2014, 
Roemmich and Bastian, 2015) and savings (Leech et al., 2018). Based on this evidence, we reasoned that the adaptations in stability control seen after the perturbations of the left leg may be transferred to aid recovery from perturbation to the right leg. One previous study that did analyse interlimb transfer of adaptations in reactive gait stability found that participants could only transfer pre-perturbation adaptations (predictive, not reactive) in gait between limbs (Bhatt and Pai, 2008), but their analysis only included the first recovery step and the perturbation was not standardised, meaning that the exact impact of the perturbation to each limb may have slightly differed. Regarding the improvements in stability observed following the two perturbations to the right leg on Day 2 , we cannot conclusively say, based on the current data, whether or not these were due to interlimb transfer of adaptations. Our post hoc analysis seems to suggest that a practice effect may have contributed to these findings (Fig. 8), but not all of the first four perturbations to each limb occurred on the same day, limiting the conclusions that can be drawn.

As can be seen in our results, individual responses to the perturbations varied. It could be argued that individual variability within and between participants may therefore influence the analysis and that by averaging repeated trials, a clearer picture of the effect of the perturbations could be gained. However, as the effects of these perturbations are so strong, we do not feel that this variability compromises the study. Analysing these initial single trials could be considered a strength in terms of ecological validity, as the variation in responses is more representative of what is seen in daily life following real, truly unexpected perturbations to gait (Allum et al., 2011).

The participants in the current study were not given any details about the nature of the perturbations, but we did consider the possibility that performance on Day 2 could be influenced by prior knowledge and experience of the task acquired on Day 1. Even though no measurable changes in gait stability during baseline walking were found in the current study, previous studies have demonstrated the beneficial effects of increased awareness of perturbations on stability recovery performance following trips (Pater et al., 2015, Oludare et al., 2018). For the eight perturbations to the left leg, the plateau of recovery steps required for re-stabilisation on Day 1 was quickly improved upon on Day 2 (Figs. 6B and 7). It is unclear if this was due to independent or combined effects of retention, savings or increased task awareness, but we can conclude that for this form of gait perturbation, "complete" adaptation on first exposure does not necessarily represent the participant's best task performance, which has implications for perturbation-based balance training programmes.

In conclusion, we have shown that young healthy adults are capable of adapting their gait in a reactive, feedback driven manner to control stability and reduce the number of steps to reach positive and baseline values of MoS, and that they can fully retain these adaptations over a one-month period. On re-exposure to the perturbations, a combination of retention and savings led to further improvements in reactive stability control above those made one month before. In contrast to our expectations, evidence of interlimb transfer of reactive gait adaptations was inconclusive. Our results show that humans utilise retention and savings in reactive gait adaptations to benefit stability, but that interlimb transfer may not be exclusively responsible for improvements following 
perturbations to the untrained limb. These findings broaden our understanding of reactive gait adaptability and have implications for future studies on gait stability and adaptability, as well as for falls prevention interventions.

\section{Methods}

\section{Participants}

Eighteen healthy adults participated in this study (eight males, 10 females; age: $24.4 \pm 2.5 \mathrm{y}$; height: $174.9 \pm 7.4 \mathrm{~cm}$; weight: $74.6 \pm 15.2 \mathrm{~kg}$ ). The participants had no self-reported history of walking difficulties, dizziness or balance problems, and had no known neuromuscular condition or injury that could affect balance or walking. Informed consent was obtained and the study was conducted in accordance with the Declaration of Helsinki. The study protocol was approved by the Maastricht University Medical Centre medical ethics committee (NL58205.068.16).

\section{Setup and Procedures}

The Computer Assisted Rehabilitation Environment Extended (CAREN; Motekforce Link, Amsterdam, The Netherlands) was used for this study, which included a dual-belt force plate-instrumented treadmill (Motekforce Link, Amsterdam, The Netherlands; 1000Hz), a 12 -camera motion capture system $(100 \mathrm{~Hz}$; Vicon Motion Systems, Oxford, UK) and a virtual environment that provided optic flow during walking. Three high definition video cameras also recorded video footage of the measurements. A safety harness system connected to an overhead frame was used at all times. Five retroreflective markers were attached to anatomical landmarks ( $\mathrm{C} 7$, left and right trochanter and left and right hallux) and the three-dimensional coordinates of these markers were tracked by the motion capture system. Each session began with walking familiarisation trials at $0.4 \mathrm{~m} \cdot \mathrm{s}^{-1}$ up to $1.8 \mathrm{~m} \cdot \mathrm{s}^{-1}$. 60s were used for each speed. Participants were then given sufficient rest (approximately two minutes) before continuing with the measurements.

The procedures for determining the stability-normalised walking speed, as well as the theoretical background and data regarding the effectiveness of this approach are described in detail elsewhere (McCrum et al., 2019b). Briefly, single two-to-three-minutelong measurements were conducted at $0.4 \mathrm{~m} \cdot \mathrm{s}^{-1}$ up to $1.8 \mathrm{~m} \cdot \mathrm{s}^{-1}$ in $0.2 \mathrm{~m} \cdot \mathrm{s}^{-1}$ intervals. During a second rest period for the participants, the stability-normalised walking speed was calculated. In order to determine the stability-normalised walking speed, the mean anteroposterior margins of stability (MoS; see below) at foot touchdown of the final 10 steps of each walking trial $\left(0.4 \mathrm{~m} \cdot \mathrm{s}^{-1}\right.$ to $\left.1.8 \mathrm{~m} \cdot \mathrm{s}^{-1}\right)$ were taken and were fitted with a second order polynomial function. For each participant, the walking speed that would result in MoS of $0.05 \mathrm{~m}$ was calculated from the function.

The gait perturbation protocol then began with three to four minutes of unperturbed walking at the stability-normalised walking speed, in order to allow participants to familiarise themselves with this speed. The participants then experienced 10 unilateral treadmill belt acceleration perturbations, each occurring every 30-90s 
without warning (the washout time periods between perturbations was the same for all subjects; Fig. 1). The first and last perturbed the right leg, while the second to ninth repeatedly perturbed the left leg (Fig. 1). The perturbation consisted of a $3 \mathrm{~m} \cdot \mathrm{s}^{-2}$ acceleration of the treadmill belt to $180 \%$ of the stability-normalised walking speed, triggered automatically (using the D-Flow software of the CAREN; Motekforce Link, Amsterdam, The Netherlands) when the vertical projection of the hallux marker of the tobe-perturbed limb became anterior to the hallux marker of the stance limb in the sagittal plane. Thereby, the belt acceleration started before foot touchdown to allow a higher magnitude of perturbation to the entire stance phase. The belt decelerated when the perturbed limb lost contact with the ground (toe-off; see below). Any consecutive foot contacts with the perturbed belt (i.e. when both the perturbed limb and the first recovery step were accelerated) were noted, but none occurred during the perturbations analysed in this study. The participants returned approximately one month later (28.4 \pm 3.4 days) and repeated the perturbation protocol. On each occasion, participants were told that they would complete a walking balance challenge lasting about 10 to 15 minutes, and that their task was to try to continue walking as normally as possible. The participants were unaware of the specifics of the perturbation protocol (i.e. limbs to be perturbed, type, number, timing, magnitude of the perturbations) and no warnings or cues were given prior to the perturbations. Note that we also made participants aware of the capacities of the CAREN system, in that it could provide perturbations via platform shifts and pitches as well as treadmill belt movements. On the second day, they were informed that they would again experience a walking balance challenge lasting about 10 to 15 minutes, but that it could be different to the first day. We asked the participants about what they perceived regarding the perturbations following the first session and no participant was able to describe the precise protocol, suggesting that knowledge of the order of the perturbations would not have influenced the performance on the second measurement day. This study did not account for lower limb dominance, but due to the bipedal nature of the task (i.e. multiple recovery steps are required) and that we observed no significant differences between the first two perturbations (to the left and right lower limbs, respectively), we do not feel that this will have had a meaningful influence on the results. In fact, no previous study has highlighted limb dominance to be a major factor in gait stability, with one study specifically investigating the issue using a forward lean-and-release task and finding no differences between limbs in young and older adults (Madigan and Lloyd, 2005).

\section{Data Processing and Margin of Stability Calculation}

The three-dimensional coordinates of the markers were filtered using a low pass second order Butterworth filter (zero-phase) with a $12 \mathrm{~Hz}$ cut-off frequency. For all steps, the foot marker anteroposterior velocity data was used to determine foot touchdown and toe-off (the frame in which the marker velocity direction switched) (Zeni et al., 2008). This was then corrected based on the average discrepancy between a force plate-determined touchdown and toe-off (with a force threshold of $50 \mathrm{~N}$ ) and the marker-determined touchdown and toe-off for all steps that contacted only one force plate. This combined method was used to be able to accurately account for foot touchdowns and toe-offs occurring in the centre of the treadmill triggering both force plates simultaneously. The 
anteroposterior MoS were calculated for the moment of foot touchdown as the anteroposterior difference between the base of support (anteroposterior distance between the hallux markers) and the extrapolated centre of mass ( $\left.\mathrm{X}_{\mathrm{CoM}}\right)$ as defined by Hof et al. (2005), adapted for the reduced kinematic model based on Süptitz et al. (2013):

$$
\mathrm{X}_{\mathrm{CoM}}=\frac{\mathrm{P}_{\text {TroL }}+\mathrm{P}_{\text {TroR }}}{2}-\mathrm{P}_{\text {HalluxP }}+\frac{0.5\left(\frac{\mathrm{V}_{\text {TroL }}+\mathrm{V}_{\text {TroR }}}{2}+\mathrm{V}_{\mathrm{C} 7}\right)+\left|\mathrm{V}_{\text {Belt }}\right|}{\sqrt{\frac{\mathrm{g}}{\mathrm{L}_{\text {Ref }}}}}
$$

where $\mathrm{P}_{\text {TroL }}, \mathrm{P}_{\text {TroR }}$ and $\mathrm{P}_{\text {HalluxP }}$ represent the trochanter and the rearmost hallux marker anteroposterior positions respectively; $\mathrm{V}_{\text {TroL }}, \mathrm{V}_{\text {TroR }}$ and $\mathrm{V}_{\mathrm{C} 7}$ are the anteroposterior velocities of the trochanter and $\mathrm{C} 7$ markers respectively; $\mathrm{V}_{\mathrm{Belt}}$ is the treadmill belt velocity; $\mathrm{g}$ is gravitational acceleration $\left(9.81 \mathrm{~m} / \mathrm{s}^{2}\right)$; and $\mathrm{L}_{\mathrm{Ref}}$ is the reference leg length. The MoS concept is one of the few well-defined and well-accepted biomechanical measures of mechanical stability of the body configuration during dynamic movement (Bruijn et al., 2013), with one study demonstrating that, during a forward loss of balance, participants who required multiple recovery steps had a negative MoS value at touchdown of the first recovery step in all cases, whereas participants who only required this one recovery step all had a positive MoS (Arampatzis et al., 2008). The MoS was calculated for the following steps: baseline for each perturbation was the mean MoS of the eleventh to second last step before each perturbation (Base); the final step before each perturbation (Pre); and the first eight recovery steps following each perturbation (Post1-8).

\section{Statistics}

Two-way repeated measures ANOVAs with perturbation number $\left(\right.$ Pert $1_{\mathrm{R}}$, Pert $2_{\mathrm{L}}$, Pert9 $9_{\mathrm{L}}$ and Pert10R, representing the first and final perturbations to each limb on each day) and step (Base, Pre, Post1-Post8) as factors with post hoc Tukey's tests for multiple comparisons were used for each day to determine the following: predictive adaptation across the perturbation protocol (Perturbation number difference in Base and Pre); acute adaptation to the perturbation on each day (Pert2L vs. Pert9L); acute interlimb transfer of adaptations on each day (Pert1R vs. Pert10R); savings in the acute recovery response to a perturbation (quicker return to baseline MoS in Day 2 Pert2 $\mathrm{L}$ than Day 1 Pert2L). Retention of adaptations over one month was investigated with a separate two-way repeated measures ANOVA with Bonferroni's multiple comparisons test (Day 1 Pert9 L vs. Day 2 Pert2L). Normality of the data was checked using the Shapiro-Wilk test and Q-Q plots. In addition to these pre-planned analyses, post hoc explorative statistical tests were conducted (see Results). Significance was set at $\alpha=0.05$. Analyses were performed using GraphPad Prism version 7.03 for Windows (GraphPad Software Inc., La Jolla, California, USA). 


\section{Supplementary Table 1. Tukey's multiple comparison test results for Base and Pre} margins of stability.

\begin{tabular}{|c|c|c|c|c|}
\hline $\begin{array}{l}\text { Tukey's multiple } \\
\text { comparisons test }\end{array}$ & Mean Diff. (m) & $95.00 \%$ CI of diff. (m) & Significant? & $\begin{array}{c}\text { Adjusted } \\
\text { P Value } \\
\end{array}$ \\
\hline \multicolumn{5}{|l|}{ Day 1 Base } \\
\hline Pert1 $_{R}$ vs. Pert2 $2_{L}$ & 0.0048 & -0.02653 to 0.03621 & No & 0.9787 \\
\hline Pert1 $_{R}$ vs. Pert9 ${ }_{L}$ & -0.0076 & -0.03892 to 0.02381 & No & 0.9254 \\
\hline Pert1R vs. Pert10R $_{R}$ & -0.0030 & -0.03439 to 0.02834 & No & 0.9946 \\
\hline Pert2 $_{L}$ vs. Pert9 & -0.0124 & -0.04376 to 0.01897 & No & 0.7385 \\
\hline Pert2 $_{L}$ vs. Pert10 $R$ & -0.0079 & -0.03923 to 0.0235 & No & 0.9167 \\
\hline Pert9 $_{\text {L vs. Pert10 }}$ & 0.0045 & -0.02684 to 0.03589 & No & 0.9824 \\
\hline \multicolumn{5}{|l|}{ Day 1 Pre } \\
\hline Pert1 $_{R}$ vs. Pert2 $2_{L}$ & 0.0044 & -0.02701 to 0.03572 & No & 0.9843 \\
\hline Pert1 $_{R}$ vs. Pert9 $L$ & -0.0073 & -0.03868 to 0.02405 & No & 0.9316 \\
\hline Pert1 $_{R}$ vs. Pert10R & -0.0088 & -0.04017 to 0.02257 & No & 0.8878 \\
\hline Pert2L vs. Pert9 ${ }_{L}$ & -0.0117 & -0.04304 to 0.01969 & No & 0.7725 \\
\hline Pert2 $_{L}$ vs. Pert10 & -0.0131 & -0.04452 to 0.01821 & No & 0.7011 \\
\hline Pert9 $_{L}$ vs. Pert10 $R$ & -0.0015 & -0.03285 to 0.02988 & No & 0.9994 \\
\hline \multicolumn{5}{|l|}{ Day 2 Base } \\
\hline Pert1 $_{R}$ vs. Pert2 $L$ & 0.0012 & -0.02562 to 0.02796 & No & 0.9995 \\
\hline Pert1 $_{R}$ vs. Pert9 ${ }_{L}$ & -0.0066 & -0.03342 to 0.02016 & No & 0.9196 \\
\hline Pert1 $_{R}$ vs. Pert10 $R$ & -0.0057 & -0.03247 to 0.02111 & No & 0.9474 \\
\hline Pert2 $_{L}$ vs. Pert9 $9_{L}$ & -0.0078 & -0.03459 to 0.01899 & No & 0.8764 \\
\hline Pert2L vs. Pert10R $_{\text {R }}$ & -0.0068 & -0.03364 to 0.01994 & No & 0.9123 \\
\hline Pert9L vs. Pert10R $_{\mathrm{R}}$ & 0.0010 & -0.02584 to 0.02774 & No & 0.9997 \\
\hline \multicolumn{5}{|l|}{ Day 2 Pre } \\
\hline Pert1 $_{R}$ vs. Pert2L & 0.0142 & -0.01262 to 0.04096 & No & 0.5229 \\
\hline Pert1 $_{\mathrm{R}}$ vs. Pert9l & -0.0024 & -0.02916 to 0.02443 & No & 0.9958 \\
\hline Pert1 $_{R}$ vs. Pert10 ${ }_{R}$ & -0.0039 & -0.03072 to 0.02286 & No & 0.9815 \\
\hline Pert2 $L$ vs. Pert9 & -0.0165 & -0.04333 to 0.01026 & No & 0.3845 \\
\hline Pert2 $_{L}$ vs. Pert10R & -0.0181 & -0.04489 to 0.008693 & No & 0.3032 \\
\hline Pert9 $_{\text {L vs. Pert10R }}$ & -0.0016 & -0.02836 to 0.02523 & No & 0.9988 \\
\hline
\end{tabular}


Supplementary Table 2. Tukey's multiple comparison test results (step comparisons) for margins of stability during perturbation recovery on Day 1.

\begin{tabular}{|c|c|c|c|c|}
\hline $\begin{array}{l}\text { Tukey's multiple } \\
\text { comparisons test }\end{array}$ & Mean Diff. (m) & $95.00 \%$ CI of diff. (m) & Significant? & $\begin{array}{c}\text { Adjusted } \\
\text { P Value } \\
\end{array}$ \\
\hline \multicolumn{5}{|l|}{ Pert1 $_{R}$} \\
\hline Base vs. Pre & 0.0017 & -0.03696 to 0.04039 & No & $>0.9999$ \\
\hline Base vs. Post1 & 0.0746 & 0.03596 to 0.1133 & Yes & $<0.0001$ \\
\hline Base vs. Post2 & 0.0856 & 0.04695 to 0.1243 & Yes & $<0.0001$ \\
\hline Base vs. Post3 & 0.1139 & 0.07519 to 0.1525 & Yes & $<0.0001$ \\
\hline Base vs. Post4 & 0.1032 & 0.06451 to 0.1419 & Yes & $<0.0001$ \\
\hline Base vs. Post5 & 0.0515 & 0.01287 to 0.09023 & Yes & 0.0011 \\
\hline Base vs. Post 6 & 0.0275 & -0.01116 to 0.06619 & No & 0.4163 \\
\hline Base vs. Post7 & 0.0171 & -0.0216 to 0.05576 & No & 0.9257 \\
\hline Base vs. Post 8 & 0.0080 & -0.03071 to 0.04665 & No & 0.9997 \\
\hline \multicolumn{5}{|l|}{ Pert2L } \\
\hline Base vs. Pre & 0.0012 & -0.03745 to 0.03991 & No & $>0.9999$ \\
\hline Base vs. Post1 & 0.0690 & 0.03032 to 0.1077 & Yes & $<0.0001$ \\
\hline Base vs. Post2 & 0.0763 & 0.0376 to 0.1149 & Yes & $<0.0001$ \\
\hline Base vs. Post3 & 0.0920 & 0.05332 to 0.1307 & Yes & $<0.0001$ \\
\hline Base vs. Post4 & 0.0921 & 0.05345 to 0.1308 & Yes & $<0.0001$ \\
\hline Base vs. Post5 & 0.0465 & 0.007785 to 0.08514 & Yes & 0.0059 \\
\hline Base vs. Post6 & 0.0189 & -0.01978 to 0.05757 & No & 0.8696 \\
\hline Base vs. Post7 & -0.0053 & -0.04401 to 0.03334 & No & $>0.9999$ \\
\hline Base vs. Post8 & 0.0056 & -0.03308 to 0.04427 & No & $>0.9999$ \\
\hline \multicolumn{5}{|l|}{ Pert9 $_{L}$} \\
\hline Base vs. Pre & 0.0020 & -0.03672 to 0.04063 & No & $>0.9999$ \\
\hline Base vs. Post1 & 0.1191 & 0.08043 to 0.1578 & Yes & $<0.0001$ \\
\hline Base vs. Post2 & 0.0743 & 0.03563 to 0.113 & Yes & $<0.0001$ \\
\hline Base vs. Post3 & 0.0541 & 0.01537 to 0.09272 & Yes & 0.0005 \\
\hline Base vs. Post4 & 0.0482 & 0.009499 to 0.08685 & Yes & 0.0034 \\
\hline Base vs. Post5 & 0.0204 & -0.01826 to 0.0591 & No & 0.8071 \\
\hline Base vs. Post6 & 0.0066 & -0.03207 to 0.04529 & No & $>0.9999$ \\
\hline Base vs. Post7 & -0.0008 & -0.03951 to 0.03785 & No & $>0.9999$ \\
\hline Base vs. Post8 & 0.0000 & -0.03866 to 0.03869 & No & $>0.9999$ \\
\hline \multicolumn{5}{|l|}{ Pert10R } \\
\hline Base vs. Pre & -0.0041 & -0.04273 to 0.03462 & No & $>0.9999$ \\
\hline Base vs. Post1 & 0.1018 & 0.06307 to 0.1404 & Yes & $<0.0001$ \\
\hline Base vs. Post2 & 0.0964 & 0.05776 to 0.1351 & Yes & $<0.0001$ \\
\hline Base vs. Post3 & 0.0943 & 0.05568 to 0.133 & Yes & $<0.0001$ \\
\hline Base vs. Post4 & 0.0883 & 0.04961 to 0.127 & Yes & $<0.0001$ \\
\hline Base vs. Post5 & 0.0446 & 0.005905 to 0.08326 & Yes & 0.0103 \\
\hline Base vs. Post6 & 0.0109 & -0.02775 to 0.0496 & No & 0.9965 \\
\hline Base vs. Post7 & 0.0007 & -0.03793 to 0.03942 & No & $>0.9999$ \\
\hline Base vs. Post 8 & -0.0087 & -0.04742 to 0.02993 & No & 0.9994 \\
\hline
\end{tabular}




\section{Supplementary Table 3. Tukey's multiple comparison test results (step comparisons) for margins of stability during perturbation recovery on Day 2.}

\begin{tabular}{|c|c|c|c|c|}
\hline $\begin{array}{l}\text { Tukey's multiple } \\
\text { comparisons test }\end{array}$ & Mean Diff. (m) & $95.00 \%$ CI of diff. (m) & Significant? & $\begin{array}{c}\text { Adjusted } \\
\text { P Value } \\
\end{array}$ \\
\hline \multicolumn{5}{|l|}{ Pert1 $_{R}$} \\
\hline Base vs. Pre & -0.0046 & -0.03759 to 0.02849 & No & $>0.9999$ \\
\hline Base vs. Post1 & 0.0987 & 0.06567 to 0.1317 & Yes & $<0.0001$ \\
\hline Base vs. Post2 & 0.1183 & 0.08531 to 0.1514 & Yes & $<0.0001$ \\
\hline Base vs. Post3 & 0.1013 & 0.06826 to 0.1343 & Yes & $<0.0001$ \\
\hline Base vs. Post4 & 0.0760 & 0.04298 to 0.1091 & Yes & $<0.0001$ \\
\hline Base vs. Post5 & 0.0290 & -0.004059 to 0.06202 & No & 0.1433 \\
\hline Base vs. Post6 & 0.0093 & -0.02379 to 0.04229 & No & 0.9967 \\
\hline Base vs. Post7 & 0.0078 & -0.02525 to 0.04082 & No & 0.9992 \\
\hline Base vs. Post 8 & -0.0082 & -0.0412 to 0.02487 & No & 0.9988 \\
\hline \multicolumn{5}{|l|}{ Pert2 $_{L}$} \\
\hline Base vs. Pre & 0.0085 & -0.02459 to 0.04149 & No & 0.9984 \\
\hline Base vs. Post1 & 0.1238 & 0.09074 to 0.1568 & Yes & $<0.0001$ \\
\hline Base vs. Post2 & 0.0843 & 0.05126 to 0.1173 & Yes & $<0.0001$ \\
\hline Base vs. Post3 & 0.0903 & 0.05724 to 0.1233 & Yes & $<0.0001$ \\
\hline Base vs. Post4 & 0.0480 & 0.01494 to 0.08102 & Yes & 0.0002 \\
\hline Base vs. Post5 & 0.0044 & -0.02859 to 0.03748 & No & $>0.9999$ \\
\hline Base vs. Post6 & -0.0060 & -0.03907 to 0.02701 & No & 0.9999 \\
\hline Base vs. Post7 & -0.0179 & -0.05089 to 0.01518 & No & 0.7847 \\
\hline Base vs. Post 8 & -0.0042 & -0.03725 to 0.02882 & No & $>0.9999$ \\
\hline \multicolumn{5}{|l|}{ Pert9 $_{L}$} \\
\hline Base vs. Pre & -0.0003 & -0.03332 to 0.03275 & No & $>0.9999$ \\
\hline Base vs. Post1 & 0.1439 & 0.1109 to 0.177 & Yes & $<0.0001$ \\
\hline Base vs. Post2 & 0.0541 & 0.02107 to 0.08714 & Yes & $<0.0001$ \\
\hline Base vs. Post3 & 0.0250 & -0.008096 to 0.05798 & No & 0.328 \\
\hline Base vs. Post4 & 0.0164 & -0.01668 to 0.04939 & No & 0.8601 \\
\hline Base vs. Post5 & 0.0034 & -0.02962 to 0.03646 & No & $>0.9999$ \\
\hline Base vs. Post6 & 0.0092 & -0.0238 to 0.04227 & No & 0.9968 \\
\hline Base vs. Post7 & -0.0030 & -0.03604 to 0.03003 & No & $>0.9999$ \\
\hline Base vs. Post8 & 0.0005 & -0.03251 to 0.03357 & No & $>0.9999$ \\
\hline \multicolumn{5}{|l|}{ Pert10 $_{R}$} \\
\hline Base vs. Pre & -0.0028 & -0.03584 to 0.03024 & No & $>0.9999$ \\
\hline Base vs. Post 1 & 0.1116 & 0.07857 to 0.1446 & Yes & $<0.0001$ \\
\hline Base vs. Post2 & 0.0548 & 0.02181 to 0.08789 & Yes & $<0.0001$ \\
\hline Base vs. Post3 & 0.0520 & 0.01897 to 0.08505 & Yes & $<0.0001$ \\
\hline Base vs. Post4 & 0.0514 & 0.01837 to 0.08445 & Yes & $<0.0001$ \\
\hline Base vs. Post5 & 0.0077 & -0.02535 to 0.04073 & No & 0.9992 \\
\hline Base vs. Post6 & 0.0056 & -0.02747 to 0.0386 & No & $>0.9999$ \\
\hline Base vs. Post7 & -0.0003 & -0.03335 to 0.03273 & No & $>0.9999$ \\
\hline Base vs. Post 8 & 0.0011 & -0.03196 to 0.03412 & No & $>0.9999$ \\
\hline
\end{tabular}


Supplementary Table 4. Tukey's multiple comparison test results (comparing perturbations to the right leg) for margins of stability during perturbation recovery on

Day 1.

\begin{tabular}{lcccc}
\hline $\begin{array}{l}\text { Tukey's multiple } \\
\text { comparisons test }\end{array}$ & Mean Diff. (m) & $\mathbf{9 5 . 0 0 \% ~ C I ~ o f ~ d i f f . ~ ( m ) ~}$ & Significant? & $\begin{array}{c}\text { Adjusted } \\
\text { P Value }\end{array}$ \\
\hline $\begin{array}{l}\text { Pert1 } \text { V }_{\text {V }} \\
\text { Pert10 }\end{array}$ & & & & \\
\hline Base & -0.0030 & -0.03439 to 0.02834 & No & 0.9946 \\
\hline Pre & -0.0088 & -0.04017 to 0.02257 & No & 0.8878 \\
\hline Post1 & 0.0241 & -0.007284 to 0.05545 & No & 0.1972 \\
\hline Post2 & 0.0078 & -0.02359 to 0.03915 & No & 0.9192 \\
\hline Post3 & -0.0225 & -0.05391 to 0.008823 & No & 0.25 \\
\hline Post4 & -0.0179 & -0.0493 to 0.01344 & No & 0.4541 \\
\hline Post5 & -0.0100 & -0.04136 to 0.02137 & No & 0.8441 \\
\hline Post6 & -0.0196 & -0.05098 to 0.01175 & No & 0.3725 \\
\hline Post7 & -0.0194 & -0.05073 to 0.012 & No & 0.3842 \\
\hline Post8 & -0.0197 & -0.05111 to 0.01162 & No & 0.3667 \\
\hline
\end{tabular}

Supplementary Table 5. Tukey's multiple comparison test results (comparing perturbations to the right leg) for margins of stability during perturbation recovery on Day 2.

\begin{tabular}{lcccc}
\hline $\begin{array}{l}\text { Tukey's multiple } \\
\text { comparisons test }\end{array}$ & Mean Diff. (m) & $\mathbf{9 5 . 0 0 \%}$ CI of diff. (m) & Significant? & $\begin{array}{c}\text { Adjusted } \\
\text { P Value }\end{array}$ \\
\hline Pert1R vs. Pert10 & & & & \\
\hline Base & -0.0057 & -0.03247 to 0.02111 & No & 0.9474 \\
\hline Pre & -0.0039 & -0.03072 to 0.02286 & No & 0.9815 \\
\hline Post1 & 0.0072 & -0.01958 to 0.03401 & No & 0.8992 \\
\hline Post2 & -0.0692 & -0.09597 to -0.04239 & Yes & $<0.0001$ \\
\hline Post3 & -0.0550 & -0.08175 to -0.02817 & Yes & $<0.0001$ \\
\hline Post4 & -0.0303 & -0.05708 to -0.003496 & Yes & 0.0195 \\
\hline Post5 & -0.0270 & -0.05376 to -0.000176 & Yes & 0.0478 \\
\hline Post6 & -0.0094 & -0.03616 to 0.01743 & No & 0.8042 \\
\hline Post7 & -0.0138 & -0.04057 to 0.01302 & No & 0.5472 \\
\hline Post8 & 0.0036 & -0.02323 to 0.03036 & No & 0.9861 \\
\hline
\end{tabular}


Supplementary Table 6. Bonferroni's multiple comparison test results for margins of stability following the first perturbation to the left lower limb on each day.

\begin{tabular}{lcccc}
\hline $\begin{array}{l}\text { Bonferroni's multiple } \\
\text { comparisons test }\end{array}$ & Mean Diff. (m) & $\mathbf{9 5 . 0 0 \% ~ C I ~ o f ~ d i f f . ~ ( m ) ~}$ & Significant? & $\begin{array}{c}\text { Adjusted } \\
\text { P Value }\end{array}$ \\
\hline Day 1 Pert2L - Day 2 Pert2 & & & & \\
\hline Base & -0.0068 & -0.04848 to 0.03482 & No & $>0.9999$ \\
\hline Pre & 0.0004 & -0.04126 to 0.04204 & No & $>0.9999$ \\
\hline Post1 & 0.0479 & 0.006305 to 0.0896 & Yes & 0.0126 \\
\hline Post2 & 0.0012 & -0.04045 to 0.04284 & No & $>0.9999$ \\
\hline Post3 & -0.0085 & -0.0502 to 0.0331 & No & $>0.9999$ \\
\hline Post4 & -0.0510 & -0.09263 to -0.009332 & Yes & 0.0061 \\
\hline Post5 & -0.0488 & -0.0905 to -0.007202 & Yes & 0.0102 \\
\hline Post6 & -0.0317 & -0.0734 to 0.009895 & No & 0.3193 \\
\hline Post7 & -0.0194 & -0.06101 to 0.02229 & No & $>0.9999$ \\
\hline Post8 & -0.0166 & -0.05829 to 0.02501 & No & $>0.9999$ \\
\hline
\end{tabular}




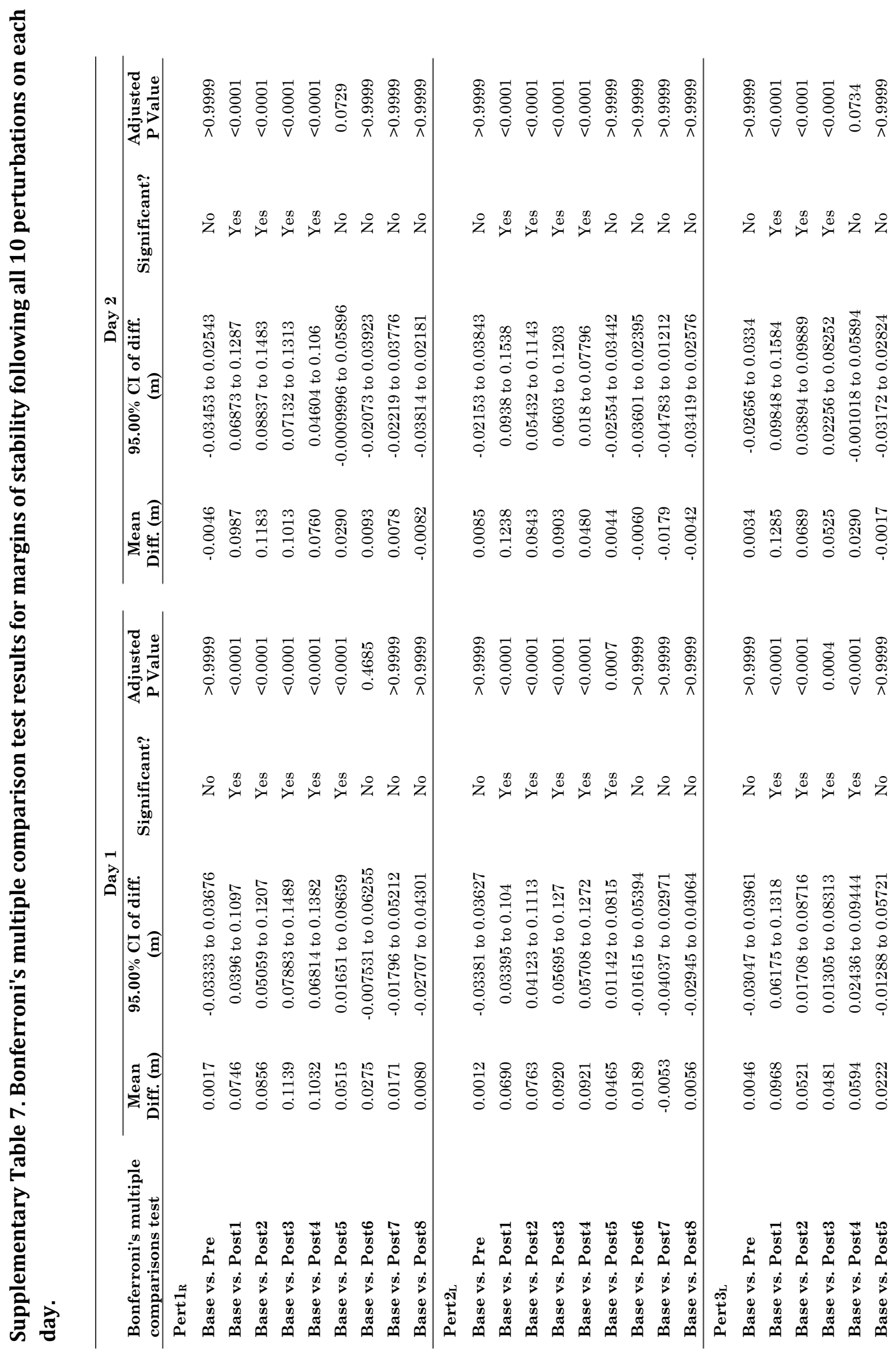




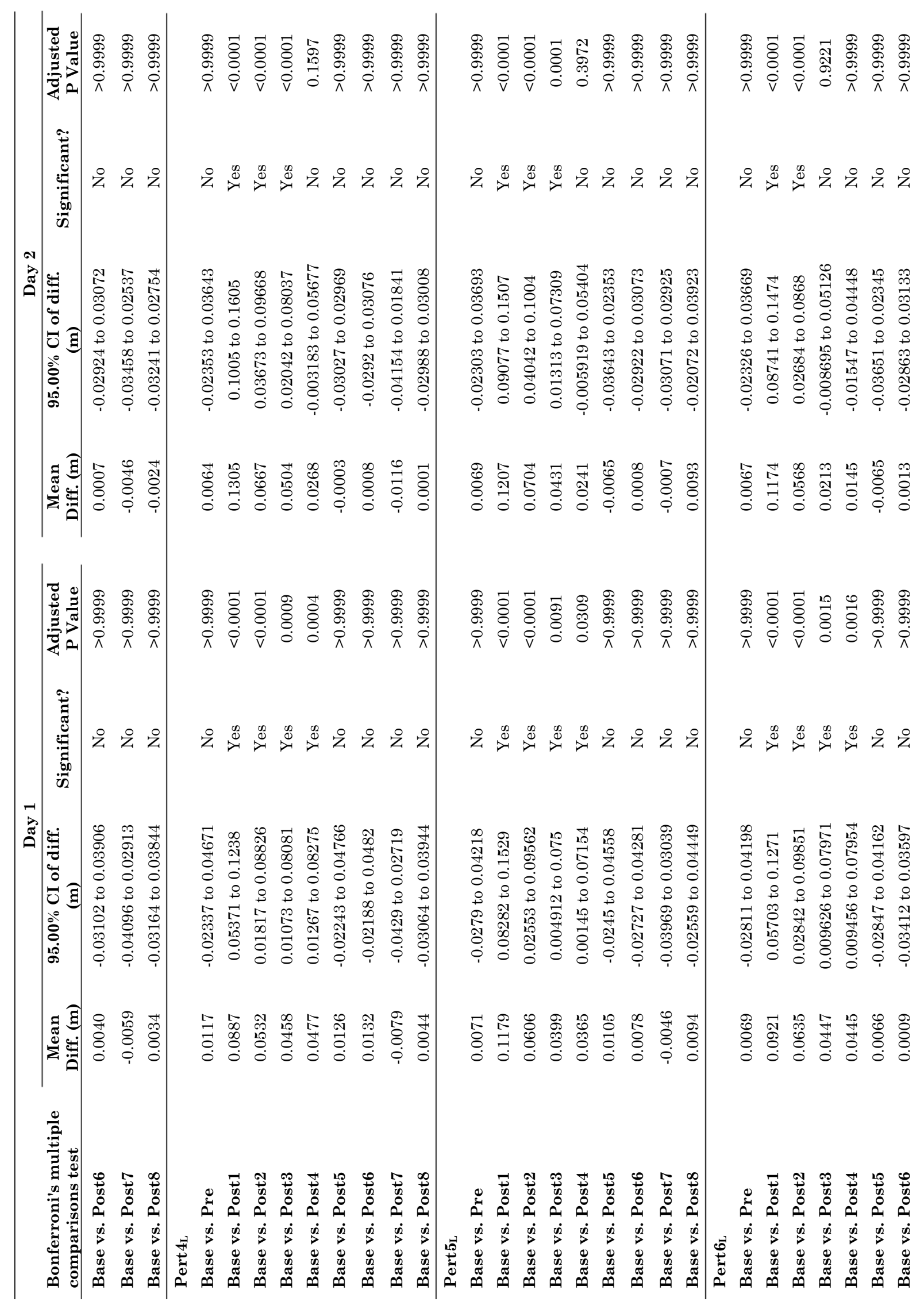




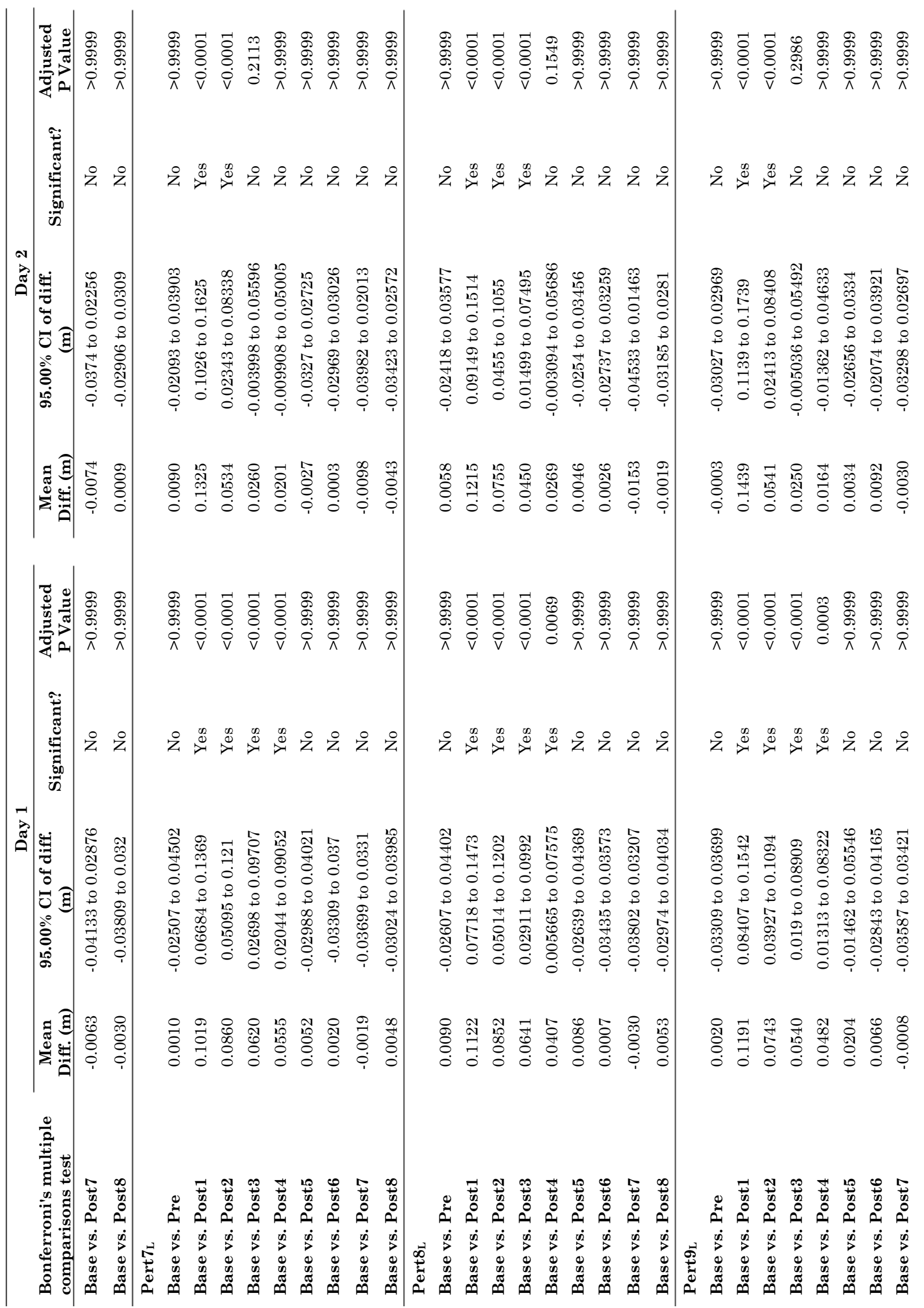




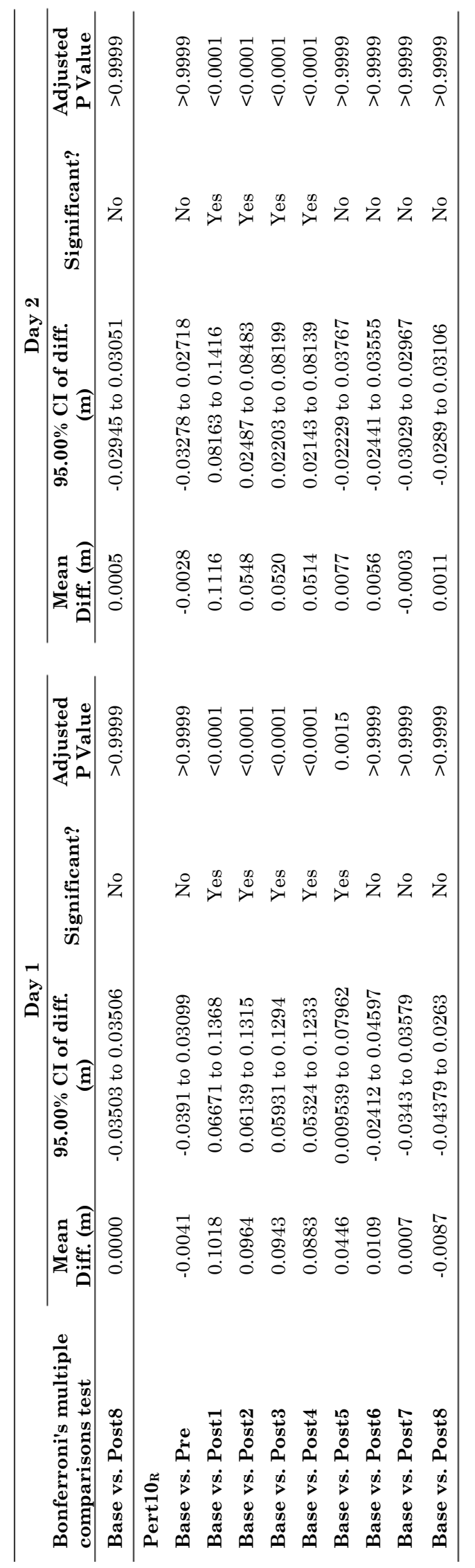




\section{2:}

\section{Older adults demonstrate interlimb transfer of reactive gait adaptations to repeated unpredictable gait perturbations}

Christopher McCrum, Kiros Karamanidis, Lotte Grevendonk, Wiebren Zijlstra, Kenneth Meijer

MCCRUM, C., KARAMANIDIS, K., GREVENDONK, L., ZIJLSTRA, W. \& MEIJER, K. 2019. Older adults demonstrate interlimb transfer of reactive gait adaptations to repeated unpredictable gait perturbations. GeroScience. doi: 10.1007/s11357-019-00130-x

(C) The Authors 2019. This work is licensed under the Creative Commons Attribution 4.0 International License. http://creativecommons.org/licenses/by/4.0/ 


\begin{abstract}
The ability to rapidly adjust gait to cope with unexpected mechanical perturbations declines with ageing. Previous studies however, have not ensured that gait stability preperturbation was equivalent across participants or age groups which may have influenced the outcomes. In this study, we investigate if age-related differences in stability following gait perturbations remain when all participants walk with equivalent stability. We also examine if interlimb transfer of gait adaptations are observed in healthy older adults, by examining if adaptation to repeated perturbations of one leg can benefit stability recovery when the other leg is perturbed. 30 young and 28 older healthy adults experienced ten unpredictable treadmill belt accelerations (the first and last applied to the right leg, the others to the left leg) during walking at their stability-normalised walking speeds (young: $1.32 \pm 0.07 \mathrm{~m} / \mathrm{s}$; older: $1.31 \pm 0.13 \mathrm{~m} / \mathrm{s}$; normalised to an average margin of stability of $0.05 \mathrm{~m}$ ). Using kinematic data, we assessed the margins of stability during unperturbed walking and the first eight post-perturbation recovery steps. Older adults required three more steps to recover during the first perturbation to each leg than the young adults. Yet, after repeated perturbations of the left leg, older adults required only one more step to recover. Interestingly, for the untrained right leg, the older adults could regain stability with three fewer steps, indicating interlimb transfer of the improvements. Age differences in reactive gait stability remain even when participants' walk with equivalent stability. Furthermore, we show that healthy older adults can transfer improvements in balance recovery made during repeated perturbations to one limb to their recovery following a perturbation to the untrained limb.
\end{abstract}




\section{Introduction}

The ability to maintain or regain gait stability following unexpected mechanical perturbations such as trips, slips or ground surface changes is negatively affected in older age (Bierbaum et al. 2010; Pavol et al. 2002; Süptitz et al. 2013) which reflects older adults' increased risk of falling during walking (Berg et al. 1997; Talbot et al. 2005). However, ageing does not greatly affect the ability to improve balance recovery responses to unexpected perturbations with repeated practice, nor the retention of these improvements over time (Bhatt et al. 2012; Epro et al. 2018a; Epro et al. 2018b; Pai et al. 2014). This has led to the development of perturbation-based balance training interventions, whereby different perturbations can be experienced and used as a training stimulus in a safe, controlled environment (Gerards et al. 2017).

There is reasonable evidence in humans that increasing motor error during locomotion (i.e. mismatch between expected sensory feedback based on the feedforward predictions of a normal gait pattern and the actual sensory feedback obtained following a perturbation to normal gait) facilitates greater or faster adaptation (Emken and Reinkensmeyer 2005; Kao et al. 2013). Motor error during a gait-like task in a stepping robot improves motor learning in young adults (Marchal-Crespo et al. 2017; MarchalCrespo et al. 2014). Furthermore, adaptation to split-belt and force-field perturbations during walking, as well as subsequent retention and savings (faster adaptation on reexposure to a perturbation) of these adaptations, often occur to a greater extent following abrupt versus gradual exposure to the perturbations (Roemmich and Bastian 2015; Torres-Oviedo and Bastian 2012; van Asseldonk et al. 2011). Additionally, experiencing large, rather than small, perturbations in an initial task leads to better generalisation to other similar locomotor tasks in both split-belt walking (Leech et al. 2018) and slipperturbed walking (Liu et al. 2016). These results indicate that older adults who experience more balance loss or difficulty recovering from gait perturbations, may experience larger motor errors (more diversion from the intended regular gait pattern) and experience a relatively larger stimulus for adaptation than younger adults completing the same gait perturbation task. In previous gait perturbation studies, transfer between similar perturbation tasks has been observed (Bieryla et al. 2007; Parijat and Lockhart 2012; Yang et al. 2013) but there is only limited evidence of interlimb transfer of reactive gait adaptations to perturbations (Bhatt and Pai 2008; McCrum et al. 2018). However, in both previous interlimb studies, only young healthy participants were included. If older adults had been exposed to similar perturbation conditions, the extent of motor errors experienced may have been much greater.

Walking speed can influence the impact of, and the response to, different perturbations (Bhatt et al. 2005; Espy et al. 2010; Pavol et al. 1999). If the same speed is used for all participants, this may result in different degrees of task difficulty (McCrum et al. 2017; McCrum et al. 2019b). In this study, we first aimed to determine if age-related differences in reactive gait stability and its adaptability in response to repeated mechanical gait perturbations are found when the participants' walking speed is normalised to gait stability. To achieve this, we applied our recently published method of walking speed normalisation which reduces inter-participant differences in gait stability (McCrum et al. 2019b) assessed by the margins of stability (MoS; Hof et al. 2005). With 
this method, multiple trials at different walking speeds are conducted, from which a speed that results in an equivalent baseline level of gait stability across participants is calculated using a polynomial function. As a result, we can infer that any differences in the response to the perturbations are not, in whole or part, artefacts of the walking speeds of the participants, but rather due to true differences in recovery responses. Based on previous work in trips leading to forward balance loss (Epro et al. 2018a; Süptitz et al. 2013), we hypothesised that older adults would require more steps to regain stability than younger adults following the initial perturbation to each leg, despite the stabilitynormalised walking speed, but that both groups would be able to adapt their gait to improve stability during the repetitions to the left leg. The second aim was to determine if interlimb transfer of these adaptations could be observed in healthy older adults, despite the limited support in young adults in our previous study (McCrum et al. 2018). Given our expectation that older adults would require more steps to regain stability than younger adults following the initial perturbation to each leg, and thereby experience greater motor error in their early responses, we hypothesised that evidence of interlimb transfer would be found in the older adults.

\section{Methods}

\section{Participants}

30 healthy young adults (12 males, 18 females; age: $24 \pm 2.5$ years; height: $173 \pm 8 \mathrm{~cm}$; weight: $71 \pm 13.9 \mathrm{~kg}$ ) and 28 healthy older adults (17 males, 11 females; age: $71 \pm 4$ years; height: $169 \pm 9.3 \mathrm{~cm}$; weight: $76 \pm 11.9 \mathrm{~kg}$ ) participated in this study. Participants were recruited via posters placed around the university and in local gyms and fitness centres. Data from 18 healthy young adults have been reported in our previous study (McCrum et al. 2018) as part of a different analysis. While this was a convenience sample taken from a larger study 4.2 powered for a different outcome, 28 to 30 participants provide sufficient power $(0.72<\beta<0.96)$ to detect the moderate to large effect sizes of interest (Cohen's $d$ of $0.5-0.7$ ) that we observed in our previous study (McCrum et al. 2018). The participants had no selfreported history of walking difficulties, dizziness or balance problems, had no known neuromuscular condition or injury that could affect balance or walking, and could walk at a regular pace for 30 minutes without assistance and without stopping. Written informed consent was obtained and the study was conducted in accordance with the Declaration of Helsinki. The study protocol was approved by the Maastricht University Medical Centre medical ethics committee.

\section{Setup and Procedures}

A dual-belt force plate-instrumented $(1000 \mathrm{~Hz})$ treadmill with a virtual environment that provided optic flow during walking (Computer Assisted Rehabilitation Environment Extended, CAREN; Motekforce Link, Amsterdam, The Netherlands) and a 12 -camera motion capture system $(100 \mathrm{~Hz}$; Vicon Motion Systems, Oxford, UK) were used in this study. Three high definition video cameras recorded video footage of the trials. Five retroreflective markers were attached to anatomical landmarks $(\mathrm{C} 7$, left and right 
trochanter and left and right hallux) and the three-dimensional coordinates of these markers were tracked by the motion capture system. Participants were secured in a safety harness system throughout the measurements.

Participants first completed 60-second walking familiarisation trials at speeds of $0.4 \mathrm{~m} / \mathrm{s}$ up to $1.8 \mathrm{~m} / \mathrm{s}$ in $0.2 \mathrm{~m} / \mathrm{s}$ increments and were given sufficient rest (approximately two minutes) before continuing with the recorded trials, comprised of two-to-three-minutelong trials (to ensure a sufficient minimum number of strides) at the same speeds. While participants rested, the stability-normalised walking speed was calculated by fitting a second order polynomial function to the mean anteroposterior MoS (see below) of the final 10 steps of each walking trial $(0.4 \mathrm{~m} / \mathrm{s}$ to $1.8 \mathrm{~m} / \mathrm{s})$ (McCrum et al. 2018; McCrum et al. $2019 \mathrm{~b})$. The theoretical background and data on the effectiveness of this approach are described elsewhere (McCrum et al. 2019b). For each participant, the walking speed that would result in MoS of $0.05 \mathrm{~m}$ was calculated from the function. The perturbation trial then began with three to four minutes of unperturbed walking at the stability-normalised walking speed, followed by 10 unannounced unilateral treadmill belt acceleration perturbations, each occurring every 30-90 seconds. Participants were told that they would complete a walking balance challenge and to try to continue walking as normally as possible. Participants were not aware of the specifics of the protocol (i.e. limbs to be perturbed, type, number, timing, magnitude of the perturbations). The first and tenth accelerations perturbed the right leg, while the second to ninth accelerations perturbed the left leg. The perturbation was a $3 \mathrm{~m} / \mathrm{s}^{2}$ acceleration of the treadmill belt to a maximum speed equal to $180 \%$ of the stability-normalised walking speed. The acceleration began when the hallux marker of the to-be-perturbed limb passed the hallux marker of the opposite foot in the sagittal plane. The belt decelerated at toe-off of the perturbed limb.

\section{Data Processing and Margin of Stability Calculation}

Data processing was conducted in MATLAB (2016a, The MathWorks, Inc., Natick). The three-dimensional coordinates of the markers were filtered using a low pass, second order, zero phase Butterworth filter with a $12 \mathrm{~Hz}$ cut-off frequency. Foot touchdown and toe-off were determined as previously described (McCrum et al. 2018; McCrum et al. 2019b). The anteroposterior MoS $\left(\operatorname{MoS}_{\mathrm{AP}}\right)$ at foot touchdown were calculated as the anteroposterior distance between the anterior boundary of the base of support (BoS) and the extrapolated centre of mass ( $\mathrm{X}_{\mathrm{CoM}}$ ) (Hof et al. 2005), adapted for our reduced kinematic model (Süptitz et al. 2013; more details, as well as the equation used for the calculation are available in Online Resource 1, eMethods). The mediolateral MoS (MoS $\mathrm{ML}_{\text {) }}$ were also calculated in a similar manner (mediolateral components instead of anteroposterior), with the exceptions that the treadmill belt velocity was not included in the estimation of CoM velocity and that the MoSML was not determined at foot touchdown, but rather the minimum MoS $\mathrm{SL}_{\mathrm{ML}}$ during the stance phase was determined (Hak et al. 2012). The MoS was calculated for the following steps: baseline for each perturbation was the mean MoS of the eleventh to second last step before each perturbation (Base); the final step before each perturbation (Pre); and the first eight recovery steps following each perturbation (Post1-8). 


\section{Statistics}

To investigate the age-related differences in the responses to novel perturbations and the adaptation to repeated perturbations to one leg, two-way repeated measures ANOVAs with group (young, older) and step (Base, Pre, Post1-Post8) as factors were conducted individually for the first, second and ninth perturbations (the first perturbation of the right leg (novel) and the first (novel) and final (adaptation) perturbations of the left "trained" leg; Pert1 $1_{\mathrm{R}}$, Pert2 $\mathrm{L}_{\mathrm{L}}$ and Pert9 $\mathrm{L}$, respectively). To evaluate any changes in the MoS $_{A P}$ during unperturbed walking that would indicate anticipatory adjustments, a repeated measure mixed model with perturbation number $\left(\right.$ Pert $1_{\mathrm{R}}$, Pert2 $\mathrm{L}_{\mathrm{L}}, \operatorname{Pert9}_{\mathrm{L}}$ and Pert10R) and age group as factors was conducted. To further investigate which MoS components might be responsible for any observed differences in the responses to the novel perturbations and the adaptation to repeated perturbations to one leg, the same ANOVAs were conducted for the BoS and $\mathrm{X}_{\mathrm{CoM}}$. Finally, the same ANOVAs were conducted for the MoSmL, as we suspected that lateral instability may also be increased in the older adults during anteroposterior perturbations (McIlroy and Maki 1996). To determine if interlimb transfer of the reactive adaptations occurred in the older adults, two-way repeated measures ANOVAs with perturbation number (Pert1 $1_{R}$ and Pert10 ${ }_{R}$ ) and step (Base, Pre, Post1-Post8) as factors were conducted for MoS $\mathrm{AP}$, BoS and $\mathrm{X}_{\mathrm{CoM}}$. For all ANOVAs, post hoc Bonferroni tests for multiple comparisons were applied. Sphericity of the data was checked and when required, outcomes were adjusted using the Geisser-Greenhouse epsilon. Significance was set at $\alpha=0.05$. Analyses were performed using GraphPad Prism version 8.02 for Windows (GraphPad Software Inc., La Jolla, California, USA).

\section{Results}

Similar to our previous work in young adults (McCrum et al., 2018a, McCrum et al., 2019a), means and standard deviations of the eleventh to second last step before the first perturbation revealed that most participants (25 of 30 young adults and 23 of 28 older adults) were within one standard deviation of the desired $0.05 \mathrm{~m} \mathrm{MoS} \mathrm{SP}_{\mathrm{AP}}$ (Fig. 1). The stability-normalised walking speeds (mean $\pm \mathrm{SD}$, range) were $1.32 \pm 0.07 \mathrm{~m} / \mathrm{s}, 1.16-1.51 \mathrm{~m} / \mathrm{s}$ for the young adults and $1.31 \pm 0.13 \mathrm{~m} / \mathrm{s}, 1.01-1.50 \mathrm{~m} / \mathrm{s}$ for the older adults.

All participants were able to recover from the gait perturbations without harness assistance. However, one older adult stopped walking after recovering from the first perturbation, leading to the treadmill stopping. This participant was therefore excluded from the analyses involving Pert $1_{\mathrm{R}}$. Two way repeated measures ANOVAs for Pert $1_{\mathrm{R}}$, Pert2 $\mathrm{L}$ and Pert9 $\mathrm{L}_{\mathrm{L}}$ revealed a significant age group effect on $\mathrm{MoS}_{\mathrm{AP}}$ for Pert $1_{\mathrm{R}}$ only $\left(\right.$ Pert $1_{\mathrm{R}}$ : $\mathrm{F}_{(1,55)}=14.11, \mathrm{P}=0.0004, \mathrm{n}_{\mathrm{p}}{ }^{2}=0.204 ;$ Pert $2_{\mathrm{L}}: \mathrm{F}_{(1,56)}=2.968, \mathrm{P}=0.0904, \mathrm{n}_{\mathrm{p}}{ }^{2}=0.050 ;$ Pert9 $\mathrm{L}_{\mathrm{L}}: \mathrm{F}_{(1,}$ $\left.{ }_{56}=0.2948, \mathrm{P}=0.5893, \mathrm{n}_{\mathrm{p}}{ }^{2}=0.005\right)$. Significant Step by Age Group interactions were found for Pert1 $1_{\mathrm{R}}$ and Pert2 $\mathrm{L}\left(\right.$ Pert1 $_{\mathrm{R}}$ : $\mathrm{F}_{(9,495)}=15.55, \mathrm{P}<0.0001, \mathrm{\eta}_{\mathrm{p}}{ }^{2}=0.220$; Pert2 $\mathrm{L}: \mathrm{F}_{(9,504)}=8.310$, $\mathrm{P}<0.0001, \eta_{\mathrm{p}}{ }^{2}=0.129$; Pert9 $\left.\mathrm{L}: \mathrm{F}_{(9,504)}=1.576, \mathrm{P}=0.1192, \mathrm{\eta}_{\mathrm{p}}{ }^{2}=0.027\right)$. Bonferroni tests for multiple comparisons (Fig. 2) revealed that, on average, older adults had MoS $\mathrm{AP}_{\mathrm{P}}$ significantly different to Base for at least three steps more than the young adults during

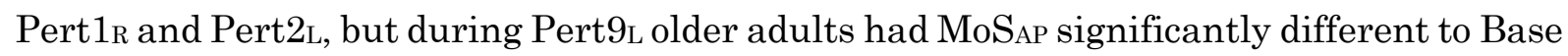
for only one step more than the young adults (five vs. four steps). The young and older 
adults improved their recovery performance following repeated perturbations (Pert2L to Pert9L) on average by two and three recovery steps, respectively. Complete Bonferroni results can be found in Online Resource 1 (eTables 1 and 2). Regarding the unperturbed walking $\mathrm{MoS}_{\mathrm{AP}}$, we did find a significant perturbation number effect $\left(\mathrm{F}_{(3,166)}=11.44\right.$, $\mathrm{P}<0.0001$ ) and Bonferroni post hoc tests revealed significant differences between Pert $1_{\mathrm{R}}$ and Pert9L, Pert2 $\mathrm{L}$ and Pert9L and between Pert2 $\mathrm{L}$ and Pert10R in the younger adults and between Pert2L and Pert9L in the older adults, but these differences ranged from $0.2 \mathrm{~cm}$ to $0.8 \mathrm{~cm}$ and were therefore not considered to have a meaningful effect on the main results.

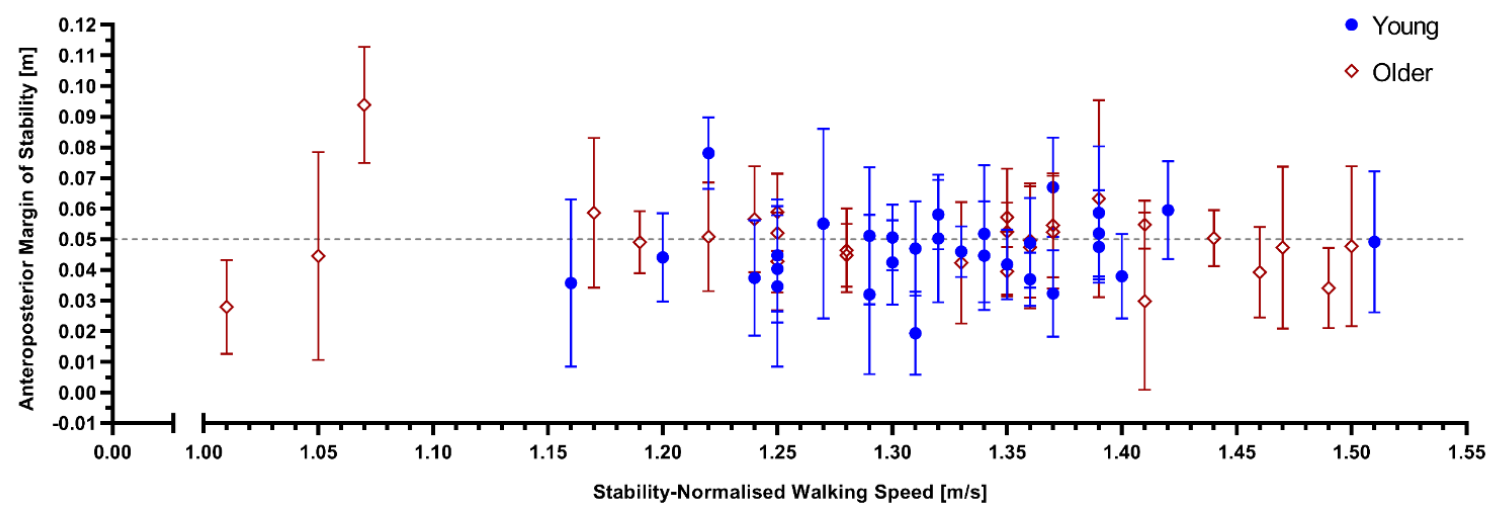

Fig. 1: Anteroposterior margins of stability (means and standard deviations) of the eleventh to second last step before the first perturbation across the individual stability-normalised walking speeds for young (blue circles) and older (red diamonds) healthy adults.
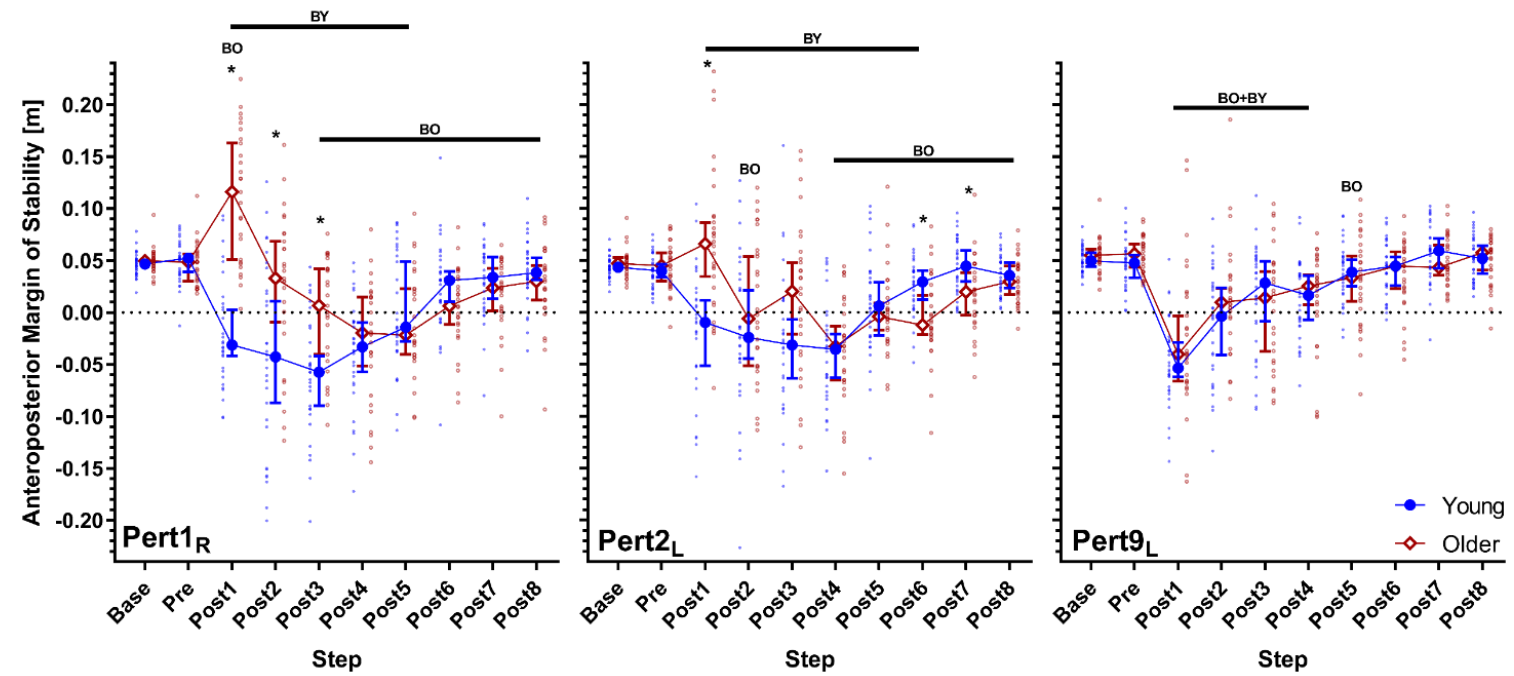

Fig. 2: Median and 95\% confidence intervals (with individual data points) of the anteroposterior margins of stability during the first, second and ninth perturbations (the first perturbations to the right and left leg and the final perturbation of the left leg; Pert $1_{\mathrm{R}}$, Pert $2_{\mathrm{L}}$, and Pert 9 , respectively) during unperturbed walking prior to each perturbation (Base), the final step prior to each perturbation (Pre) and the first eight recovery steps following the perturbations (Post1-8) for young and older adults. BO and BY: Significant difference to Base for older and young adults, respectively $(\mathrm{P}<0.05)$. * Significant difference between young and older adults $(\mathrm{P}<0.05)$. 
Two-way repeated measures ANOVAs for Pert1R, Pert2L and Pert9L revealed significant age group effects on BoS for Pert1R, Pert2L and Pert9L (Pert1R: $F_{(1,55)}=7.862$, $\mathrm{P}=0.007, \eta_{\mathrm{p}}{ }^{2}=0.125 ;$ Pert2L: $\mathrm{F}_{(1,56)}=11.75, \mathrm{P}=0.0011, \eta_{\mathrm{p}}{ }^{2}=0.173 ;$ Pert $\mathrm{L}_{\mathrm{L}}: \mathrm{F}_{(1,56)}=9.078$, $\mathrm{P}=0.0039, \mathrm{n}_{\mathrm{p}}{ }^{2}=0.139$; Fig. 3). Significant Step by Age Group interactions were found for Pert1 $1_{\mathrm{R}}$ Pert2 $\mathrm{L}_{\mathrm{L}}$ and Pert9 (Pert1 $_{\mathrm{R}}: \mathrm{F}_{(9,495)}=3.160, \mathrm{P}=0.001, \mathrm{n}_{\mathrm{p}}{ }^{2}=0.054$; Pert2 $\mathrm{L}_{\mathrm{L}}: \mathrm{F}_{(9,504)}=7.281$, $\mathrm{P}<0.0001, \mathrm{n}_{\mathrm{p}}{ }^{2}=0.115$; Pert9 $\mathrm{L}_{\mathrm{L}}: \mathrm{F}_{(9,504)}=1.987, \mathrm{P}=0.0389, \mathrm{n}_{\mathrm{p}}{ }^{2}=0.034$; Fig. 3). Bonferroni tests for multiple comparisons (Fig. 3) revealed that, on average, older adults had returned to BoS values not significantly different to Base by Post 4 during each of the analysed perturbations. Older adults had a significantly smaller BoS than young adults during Post2 to Post4 for Pert2L, and Post3 and Post4 for Pert9L. For XCoM, the ANOVAs revealed significant age group effects (Pert1 $: \mathrm{F}_{(1,55)}=16.26, \mathrm{P}=0.0002, \mathrm{\eta}_{\mathrm{p}}{ }^{2}=0.228$; Pert2L: $\mathrm{F}_{(1}$, $\left.{ }_{56}\right)=15.64, \mathrm{P}=0.0002, \eta_{\mathrm{p}}{ }^{2}=0.218$; Pert9L: $\mathrm{F}_{(1,56)}=9.140, \mathrm{P}=0.0038, \eta_{\mathrm{p}}{ }^{2}=0.140$; Fig. 3) and Step by Age Group interactions (Pert1R: $\mathrm{F}_{(9,495)}=10.45, \mathrm{P}<0.0001, \eta_{\mathrm{p}}{ }^{2}=0.160 ;$ Pert2L: $\mathrm{F}_{(9}$, 504) $=11.84, \mathrm{P}<0.0001, \eta_{\mathrm{p}}{ }^{2}=0.175 ;$ Pert9 $\mathrm{L}: \mathrm{F}_{(9,504)}=2.440, \mathrm{P}=0.0101, \mathrm{\eta}_{\mathrm{p}}{ }^{2}=0.042$; Fig. 3) for Pert1 $1_{R}$, Pert2 $2_{\mathrm{L}}$ and Pert9 $\mathrm{L}$. Bonferroni tests for multiple comparisons revealed that $\mathrm{X}_{\mathrm{CoM}}$ significantly differed between older and young adults from Post1 to Post4 for Pert $1_{\mathrm{R}}$ and Pert2L (Fig. 3). Complete Bonferroni results for the BoS and $\mathrm{X}_{\mathrm{CoM}}$ can be found in Online Resource 1 (eTables 3 to 6). Results regarding the MoS $\mathrm{SL}_{\mathrm{ML}}$ can be found in Online Resource 1 (eResults, eFigure 1, eTables 7 and 8).

Regarding the investigation of interlimb transfer in the older adults (Pert1 $1_{\mathrm{R}}$ and Pert10R), no significant perturbation number effects were found for MoS $\mathrm{SP}_{\mathrm{AP}}$ or MoS $\mathrm{SL}_{\mathrm{ML}}$ $\left(\mathrm{MoS}_{\mathrm{AP}}: \mathrm{F}_{(1,26)}=2.634, \mathrm{P}=0.1167, \mathrm{\eta}_{\mathrm{p}}^{2}=0.092 ; \mathrm{MoS}_{\mathrm{ML}}: \mathrm{F}_{(1,26)}=0.03025, \mathrm{P}=0.8633, \mathrm{\eta}_{\mathrm{p}}^{2}=0.001\right.$; Fig. 4). However, significant perturbation number effects were found for BoS and $\mathrm{X}_{\mathrm{CoM}}$ (BoS: $\mathrm{F}_{(1,26)}=9.104, \mathrm{P}=0.0056, \eta_{\mathrm{p}}^{2}=0.259 ; \mathrm{X}_{\mathrm{CoM}}: \mathrm{F}_{(1,26)}=18.32, \mathrm{P}=0.0002, \eta_{\mathrm{p}}{ }^{2}=0.413$; Fig. 4), along with significant perturbation number by step interactions for MoS $\mathrm{AP}, \mathrm{BoS}$ and $\mathrm{X}_{\mathrm{CoM}}$, but not MoSML $\left(\operatorname{MoS}_{\mathrm{AP}}: \mathrm{F}_{(4.150,107.9)}=16.42, \hat{\varepsilon}=0.4611, \mathrm{P}<0.0001, \eta_{\mathrm{p}}{ }^{2}=0.387 ; \mathrm{BoS}: \mathrm{F}_{(3.029}\right.$, 78.74) $=5.480, \hat{\varepsilon}=0.3365, \mathrm{P}=0.0017, \mathrm{\eta}_{\mathrm{p}}^{2}=0.174 ; \mathrm{X}_{\mathrm{CoM}}: \mathrm{F}_{(3.920,101.9)}=12.30, \hat{\varepsilon}=0.4355, \mathrm{P}<0.0001$, $\eta_{\mathrm{p}}{ }^{2}=0.321$; MoS $\mathrm{ML}_{\mathrm{L}}: \mathrm{F}_{(4.056,105.5)}=0.6885, \hat{\varepsilon}=0.4507, \mathrm{P}=0.6035, \mathrm{\eta}_{\mathrm{p}}{ }^{2}=0.026$; Fig. 4). Bonferroni tests for multiple comparisons are indicated in Fig. 4 and revealed that during Pert $1_{\mathrm{R}}$, the older adults did not return to $\mathrm{MoS}_{\mathrm{AP}}$ values not significantly different to Base during the eight analysed recovery steps, whereas during Pert10R, they reached this point by Post6. During Pert $1_{\mathrm{R}}$, Post1 and Post2 had significantly greater MoS $\mathrm{AP}_{\mathrm{P}}$ than during Pert10 $\mathrm{R}$, but significantly lower $\mathrm{MoS}_{\mathrm{AP}}$ during Post8. BoS was significantly smaller during Pert1 $\mathrm{R}$ than Pert10 $\mathrm{R}$ at Post2. This coincided with significant differences between Pert1 $\mathrm{R}$ and Pert10R in $\mathrm{X}_{\mathrm{CoM}}$ at Post1, Post2 and Post3, with more anterior $\mathrm{X}_{\mathrm{CoM}}$ during Pert10R. No significant differences in MoSml were observed between Pert1R and Pert10R. Complete Bonferroni results for the examination of interlimb transfer can be found in Online Resource 1 (eTables 9 to 16). 


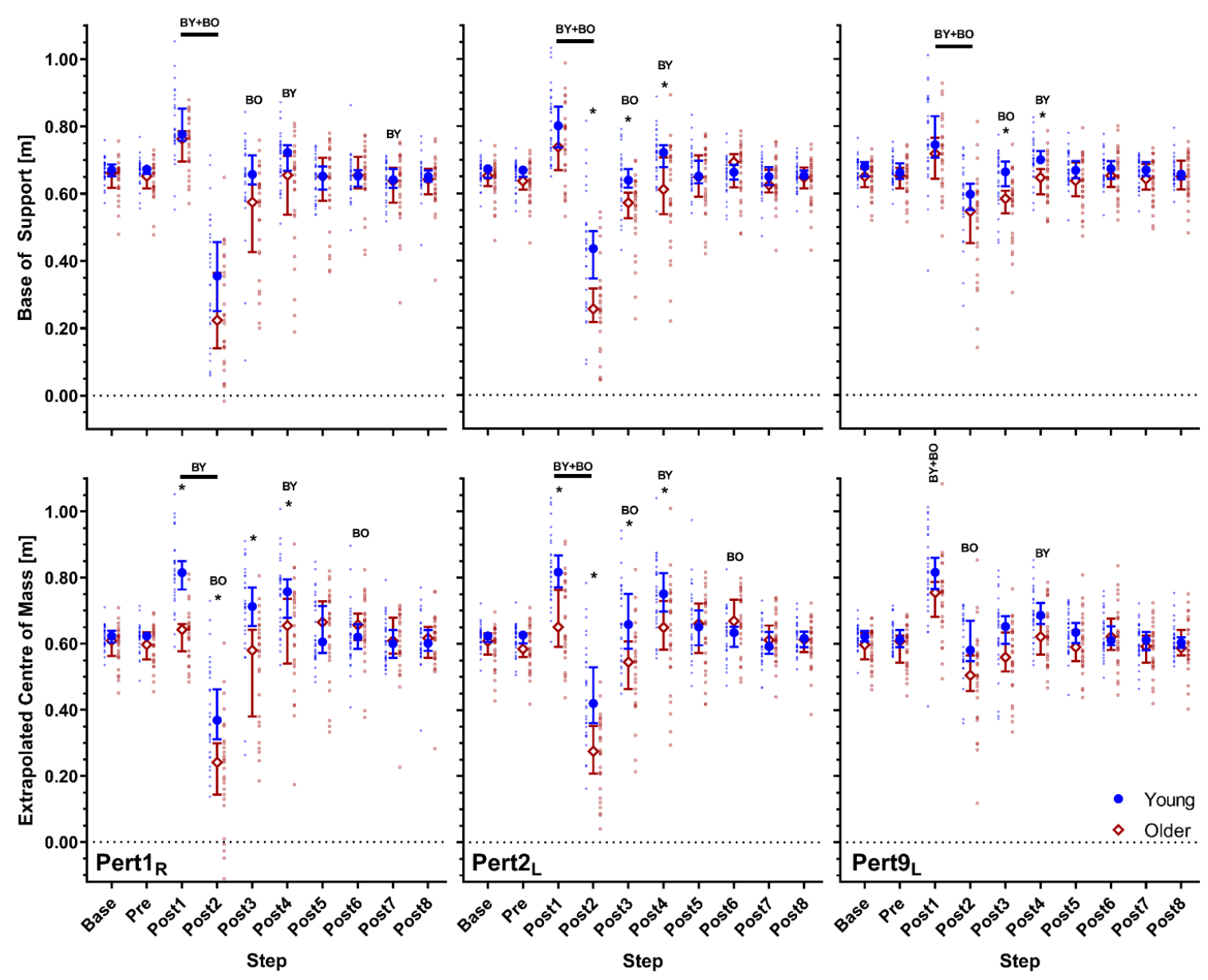

Fig. 3: Median and 95\% confidence intervals (with individual data points) of the anteroposterior base of support and extrapolated centre of mass during the first, second and ninth perturbations (the first perturbations to the right and left leg and the final perturbation of the left leg; Pert $1_{\mathrm{R}}$, Pert $2_{\mathrm{L}}$, and Pert $9_{\mathrm{L}}$, respectively) during unperturbed walking prior to each perturbation (Base), the final step prior to each perturbation (Pre) and the first eight recovery steps following the perturbations (Post1-8) for young and older adults. BO and BY: Significant difference to Base for older and young adults, respectively $(\mathrm{P}<0.05)$. *: Significant difference between young and older adults $(\mathrm{P}<0.05)$.

\section{Discussion}

In the current study, we aimed to determine whether age-related differences in reactive gait stability and its adaptability in response to repeated mechanical gait perturbations are found when the participants' walking speed is normalised to gait stability, and whether evidence of interlimb transfer of these adaptations can be observed in healthy older adults. We hypothesised that older adults require more steps to regain stability than younger adults following the initial perturbation to each leg, despite the stabilitynormalised walking speed, but that both groups would be able to adapt their gait to improve stability over the repetitions to the left leg. These hypotheses were confirmed, as the older adults required approximately three more steps to regain stability during the first perturbations to each leg than the young adults and after repeated perturbations of 

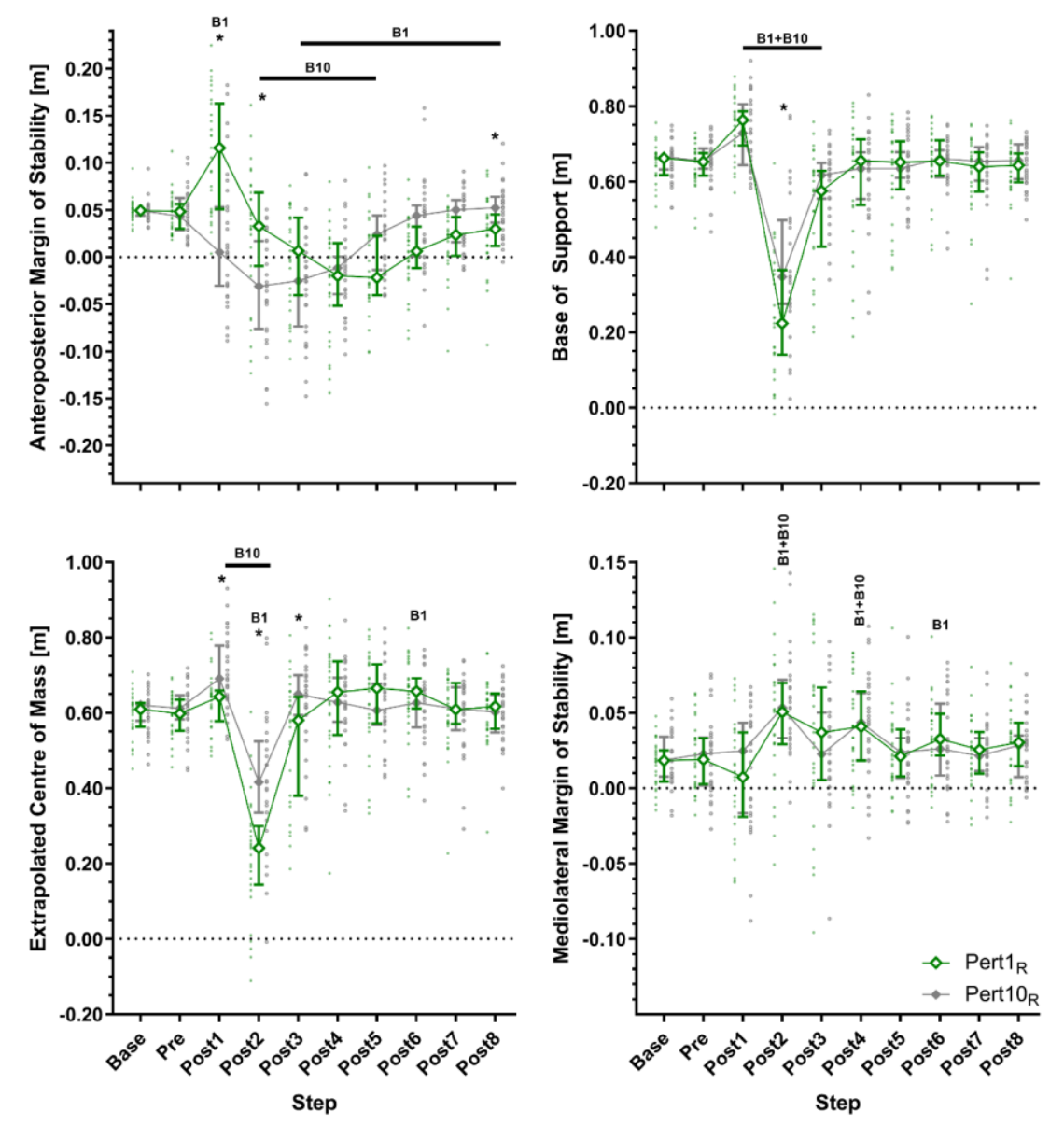

Fig. 4: Median and 95\% confidence intervals (with individual data points) of the anteroposterior margins of stability, base of support, extrapolated centre of mass and mediolateral margins of stability during the first and tenth perturbations (the first and final perturbations to the right leg; Pert $1_{\mathrm{R}}$ and Pert $10_{\mathrm{R}}$, respectively) including unperturbed walking prior to each perturbation (Base), the final step prior to each perturbation (Pre) and the first eight recovery steps following the perturbations (Post1-8) for older adults. B1 and B10: Significant difference to Base for Pert $1_{R}$ and Pert $10_{R}$, respectively $(P<0.05)$. *: Significant difference between Pert $1_{R}$ and Pert10 $(\mathrm{P}<0.05)$.

the left leg, required approximately three fewer steps to recover than during the first perturbations and were not significantly different to the young adults at any recovery step. These findings confirm previous studies in older adults using slip, trip and surface compliance perturbations (Bhatt et al. 2012; Bierbaum et al. 2010; Bierbaum et al. 2011; Epro et al. 2018a; Epro et al. 2018b; Pai et al. 2014) and extend these to treadmill belt acceleration perturbations during which the walking speed is normalised to stability, ensuring equivalent baseline gait stability and task difficulty. We also hypothesised that evidence of interlimb transfer would be found in the older adults due to them experiencing greater motor error in their early responses. This hypothesis was confirmed, as we found a three-step improvement in the steps to reach MoSAP values not significantly different to 
Base, as well as a more anterior $\mathrm{X}_{\mathrm{Com}}$ position during Post1 to Post3 in Pert10R compared to Pert1R.

Ageing has repeatedly been shown to be associated with poorer performance in regaining stability following unexpected gait perturbations (Bierbaum et al. 2010; Pavol et al. 2002; Süptitz et al. 2013). However, as previously described, potential differences in gait as a result of the walking speed choices in previous studies may have affected these findings (McCrum et al. 2017; McCrum et al. 2019b). The current study confirms and consolidates previously reported age-related differences in reactive gait stability, as age differences were observed despite the use of individual stability-normalised walking speeds. We found that these age differences in $\mathrm{MoS}_{\mathrm{AP}}$ were the result of significantly smaller $\mathrm{X}_{\mathrm{CoM}}$ during the first four recovery steps following the first two perturbations and to a lesser extent, smaller BoS during the second to fourth recovery steps following the first and second perturbations. These results indicate that the older adults responded to the treadmill belt acceleration perturbation with a more posterior $\mathrm{X}_{\mathrm{CoM}}$ and smaller BoS than young adults, delaying their stability recovery. This differs to what we have previously observed using a cable-trip setup, where the differences have been observed in the BoS (Epro et al. 2018a; McCrum et al. 2016; McCrum et al. 2014), reflecting the differences in perturbation type. By the final perturbation of the trained leg, more posterior $\mathrm{X}_{\mathrm{CoM}}$ (not significant) and smaller BoS values were still visible in the older adults compared to the young adults, but these no longer led to significantly different MoS $\mathrm{AP}_{\mathrm{P}}$ values. Multiple studies have demonstrated the ability of healthy adults to reactively adapt gait in response to repeated perturbations (Bhatt et al. 2012; Epro et al. 2018a; Epro et al. 2018b; Pai et al. 2014) and the current study confirms these findings in a treadmill belt acceleration paradigm with stability-normalised walking speeds and walking speed normalised perturbations. Therefore, we can conclude that potential differences in the initial gait stability or perturbation characteristics likely do not play a large role in whether older adults adapt their response to repeated perturbations.

We previously found little support in young adults for interlimb transfer of reactive gait adaptations following the same protocol as the current study (McCrum et al. 2018). However, we expected that older adults would require more steps to regain stability than younger adults following the initial perturbation to each leg, and thereby they would experience greater motor error in their early responses that may stimulate interlimb transfer. Our results confirmed this expectation, as we found a three-step improvement in the steps to reach $\mathrm{MoS}_{\mathrm{AP}}$ values not significantly different to Base from Pert1 $1_{\mathrm{R}}$ to Pert10 . In addition, perturbation number effects on $\mathrm{BoS}$ and $\mathrm{X}_{\mathrm{CoM}}$ were found. The older adults appeared to respond to Pert1 $\mathrm{R}$ with a more posterior $\mathrm{X}_{\mathrm{CoM}}$ at Post1 than in Pert10R, and with a smaller $\mathrm{BoS}$ and posterior $\mathrm{X}_{\mathrm{CoM}}$ at Post2. This alteration in recovery strategy during Post1-3 resulted in the three-step reduction in reaching MoS $\mathrm{AP}_{\mathrm{P}}$ values not significantly different to Base. Therefore, it appears that both the overall recovery performance and the altered movement strategy were transferred to the untrained leg. However, as it is well established that learning following such perturbations can occur within a single trial (Liu et al. 2017; Marigold and Patla 2002), we conducted an additional post hoc analysis to determine the extent of trial-to-trial learning for the first two perturbations to the left leg (Pert2 $\mathrm{L}$ and Pert3 $3_{\mathrm{L}}$ ), in order to determine if the changes from Pert1 $\mathrm{R}$ to Pert10 $\mathrm{R}$ could be 
explained by such a phenomenon. Briefly, we did find differences between Pert2L and Pert3L but not to the extent that the differences between Pert1 $\mathrm{R}$ and Pert10 $\mathrm{R}$ could be largely accounted for by the single trial effect. The complete results of this additional analysis can be found in Online Resource 1 (eResults, eFigure 2, eTables 17 and 18).

An interesting finding of the current study was that the older adults during Pert $1 \mathrm{R}$ and Pert2L demonstrated an increase, rather than a decrease in stability at Post1, whereas the young adults during all perturbations and the older adults during Pert9 ${ }_{\mathrm{L}}$ and Pert10 R (Figs. 2 and 4) demonstrated a decrease in stability. This increase was caused by a more posterior $\mathrm{X}_{\mathrm{CoM}}$ during Post1 in Pert1 $1_{\mathrm{R}}$ and Pert $2_{\mathrm{L}}$, but not a difference in BoS, implying that trunk motion was at least partly responsible. Future work could further investigate this using a kinematic model more suited to assessing trunk motion in detail. We speculate that this may be one potential reason for the observed interlimb transfer of balance recovery performance. While the lower limbs may play very specific roles in perturbation recovery during the first recovery step (i.e. push-off versus swing and placing of the foot), the role of the trunk may be more generalisable across perturbations to different limbs (i.e. counter-rotation to forward balance loss). This may also explain why no clear interlimb transfer occurred in our previous study (McCrum et al. 2018) because young adults appear to have a more anterior $\mathrm{X}_{\text {CoM }}$ position than older adults (Fig. 3). Regarding our analyses of MoSmL, the results did not reveal any substantial differences with age and these are discussed in Online Resource 1 (eDiscussion).

A limitation of the current work is that it is unclear if these findings would generalise to populations with reduced locomotor function and it is these groups that potentially could benefit most from perturbation-based balance training programmes (Gerards et al. 2017). Therefore, interlimb and intertask transfer of adaptations in reactive balance control and the generalisability of these improvements to daily life should be further explored. It could be argued that leg dominance may have affected the results, but due to the bipedal nature of the task, we think this is unlikely. Only one study has specifically investigated the effect of limb dominance on recovery from sudden balance loss and found no differences in performance between stepping with the dominant and nondominant limbs in young and older adults (Madigan and Lloyd 2005). Another limitation worth considering is that despite the evidence provided here that interlimb transfer can occur during a single short perturbation session, this does not necessarily imply that this will be retained over time, as perturbation dose appears to be related to the degree of retention possible (König et al. 2019; Liu et al. 2017).

In conclusion, the current results show that healthy older adults have a decreased ability to cope with unpredictable gait perturbations compared with younger adults, even when their walking speeds are normalised to an equivalent stability value. However, as previous studies have also shown, older healthy adults are capable of reactively adapting their gait to improve their stability following repeated gait perturbations and can then perform similarly to young adults. The current study provides evidence that older adults can transfer improvements in the number of steps required for balance recovery following repeated perturbations to one limb to their recovery following a perturbation to the untrained limb, which in this study was mostly due to an alteration in the $\mathrm{X}_{\mathrm{Com}}$ position, rather than in the BoS. 


\section{Acknowledgements}

The authors thank Paul Willems, Wouter Bijnens and Marissa Gerards for their support during this project. 


\section{Online Resource 1: Supplementary Material}

\section{eMethods}

For this study, we used the margins of stability (MoS) concept (Hof et al. 2005), one of only a few clearly defined and accepted measures of the mechanical stability of the body configuration during gait (Bruijn et al. 2013), useful for assessing changes in gait stability due to mechanical perturbations and balance loss. The anteroposterior MoS (MoSAP) were calculated at foot touchdown as the difference between the anterior boundary of the base of support (anteroposterior component of the hallux marker projection to the ground) and the extrapolated centre of mass as defined by Hof et al. (2005), adapted for our reduced kinematic model based on Süptitz et al. (2013), as follows:

$$
\mathrm{X}_{\mathrm{CoM}}=\frac{\mathrm{P}_{\text {TroL }}+\mathrm{P}_{\text {TroR }}}{2}-\mathrm{P}_{\text {HalluxP }}+\frac{0.5\left(\frac{\mathrm{V}_{\text {TroL }}+\mathrm{V}_{\text {TroR }}}{2}+\mathrm{V}_{\mathrm{C} 7}\right)+\left|\mathrm{V}_{\text {Belt }}\right|}{\sqrt{\frac{\mathrm{g}}{\mathrm{L}_{\text {Ref }}}}}
$$

where $\mathrm{P}_{\text {TroL }}, \mathrm{P}_{\text {TroR }}$ and $\mathrm{P}_{\text {HalluxP }}$ are the trochanter and the posterior hallux marker anteroposterior positions respectively; $\mathrm{V}_{\text {TroL }}, \mathrm{V}_{\text {TroR }}$ and $\mathrm{V}_{\mathrm{C} 7}$ are the anteroposterior velocities of the trochanter and $\mathrm{C} 7$ markers respectively; $\mathrm{V}_{\mathrm{Belt}}$ is the treadmill belt velocity; $\mathrm{g}$ is gravitational acceleration $\left(9.81 \mathrm{~m} / \mathrm{s}^{2}\right)$; and $\mathrm{L}_{\mathrm{Ref}}$ is the reference leg length. This reduced kinematic model was previously shown to be suitable for assessing the MoS and it's components during unperturbed and perturbed treadmill walking in young, middle and older-aged healthy adults, with high correlations and no clear differences compared to a full kinematic model (Süptitz et al. 2013).

\section{eResults}

Regarding the MoS $\mathrm{SL}_{\mathrm{ML}}$, the two way repeated measures ANOVAs for Pert1 $1_{\mathrm{R}}$, Pert $2_{\mathrm{L}}$ and Pert9 ${ }_{L}$ revealed a significant age effect for Pert1 $1_{R}$ and Pert9 ${ }_{L}$, but not for Pert2 $2_{L}$ (Pert1 : $\mathrm{F}_{(1,55)}=4.973, \mathrm{P}=0.0298, \eta_{\mathrm{p}}{ }^{2}=0.083 ;$ Pert2 ${ }_{\mathrm{L}}: \mathrm{F}_{(1,56)}=2.031, \mathrm{P}=0.1597, \eta_{\mathrm{p}}{ }^{2}=0.035 ;$ Pert9 $\mathrm{L}_{\mathrm{L}}: \mathrm{F}_{(1,}$ 56) $=4.110, \mathrm{P}=0.0474, \mathrm{n}_{\mathrm{p}}{ }^{2}=0.068$; eFig. 1). Significant Age Group by Step interactions were found for Pert1R, Pert2L and Pert9L (Pert1R: $\mathrm{F}_{(9,495)}=1.965, \mathrm{P}=0.0415, \mathrm{n}_{\mathrm{p}}{ }^{2}=0.034 ;$ Pert2L: $\mathrm{F}_{(9}$, ${ }_{504)}=2.254, \mathrm{P}=0.0177, \mathrm{\eta}_{\mathrm{p}}{ }^{2}=0.039 ;$ Pert9 $\mathrm{L}_{\mathrm{L}} \mathrm{F}(9,504)=3.610, \mathrm{P}=0.0002, \mathrm{\eta}_{\mathrm{p}}{ }^{2}=0.061 ;$ eFig. 1). Bonferroni tests for multiple comparisons are indicated in eFig. 1 and revealed two significant difference between the age groups at Post4 during Pert1R, and significant differences at Post5 and Post6 during Pert9L but no major differences were identified pre and post repetition of the left limb perturbations, with both age groups showing significantly increased MoS $\mathrm{SL}_{\mathrm{L}}$ at Post2 and Post3 during Pert2L and Pert9L. Complete Bonferroni results can be found in eTables 7 and 8 .

Regarding the investigation of the potential influence of the single trial effect on the interlimb transfer results in the older adults, a two-way repeated measures ANOVA with perturbation number (Pert2L and Pert3L) and step (Base, Pre, Post1-Post8) as factors with post hoc Bonferroni tests for multiple comparisons were conducted for MoS AP. Similar to the interlimb analysis, no significant perturbation number effect was found $\left(\mathrm{F}_{(1}\right.$, 26) $=1.681, \mathrm{P}=0.2061)$, although the size of the effect $\left(\mathrm{n}_{\mathrm{p}}{ }^{2}=0.061\right)$ was smaller than that of 
the interlimb analysis $\left(\eta_{\mathrm{p}}^{2}=0.092\right)$. Bonferroni tests for multiple comparisons revealed that during Pert2L, the older adults did not return to MoSAP values not significantly different to Base during the eight analysed recovery steps, whereas for Pert3L this was achieved at Post7, resulting in an average of two steps improvement (eFig. 2). Complete Bonferroni results can be found in eTables 17 and 18 .

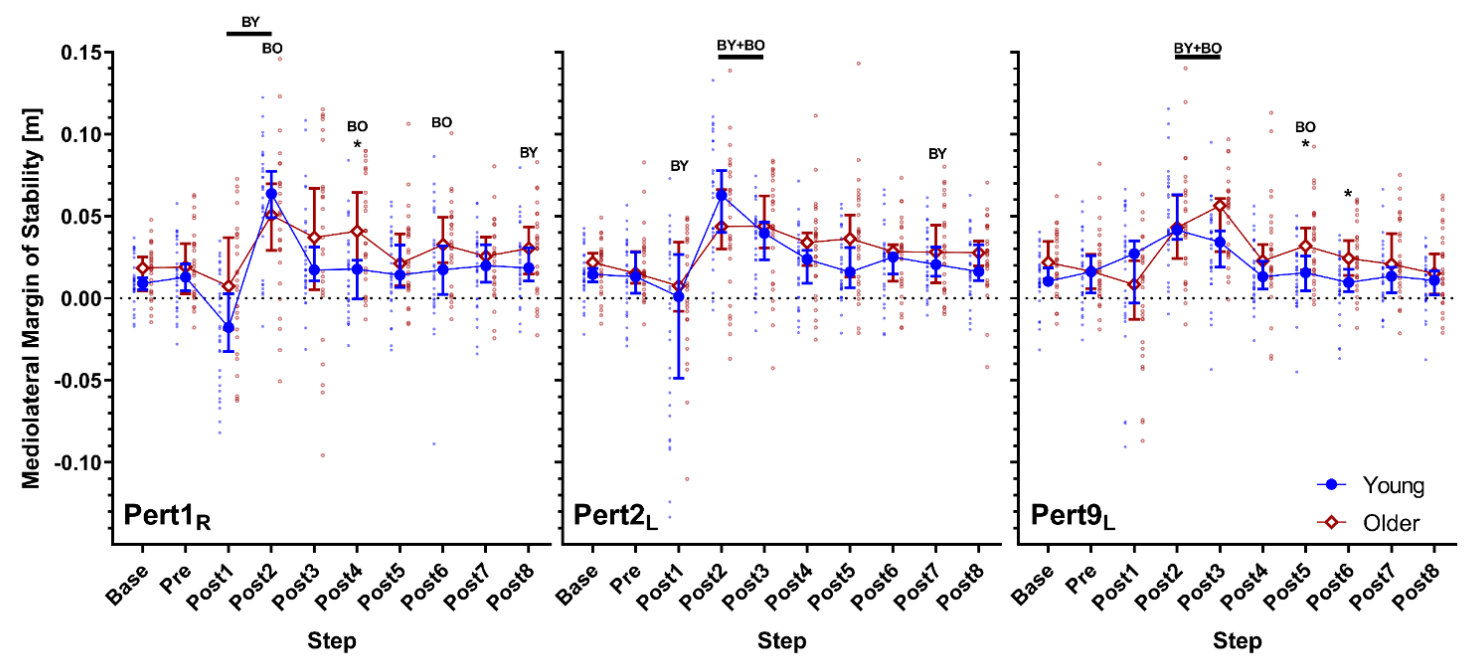

eFigure 1: Median and 95\% confidence intervals (with individual data points) of the mediolateral margin of stability during the first, second and ninth perturbations (the first perturbations to the right and left leg and the final perturbation of the left leg; Pert $1_{\mathrm{R}}$, Pert $2_{\mathrm{L}}$, and Pert9 9 , respectively) during unperturbed walking prior to each perturbation (Base), the final step prior to each perturbation (Pre) and the first eight recovery steps following the perturbations (Post1-8) for young and older adults. BO and BY: Significant difference to Base for older and young adults, respectively $(\mathrm{P}<0.05)$. : Significant difference between young and older adults $(\mathrm{P}<0.05)$.

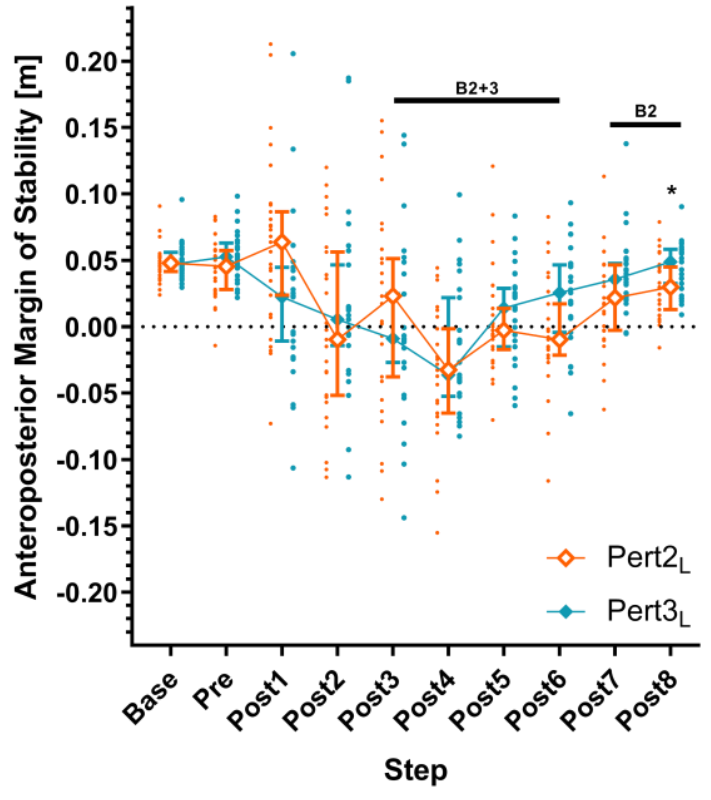

eFigure 2: Median and 95\% confidence intervals (with individual data points) of the anteroposterior margin of stability during the second and third perturbations (Pert2 $\mathrm{L}$ and Pert3 $\mathrm{L}_{\mathrm{L}}$ respectively) including unperturbed walking prior to each perturbation (Base), the final step prior to each perturbation (Pre) and the first eight recovery steps following the perturbations (Post1-8) for the older adults. B2 and B3: Significant difference to Base for Pert $2_{\mathrm{L}}$ and Pert $3_{\mathrm{L}}$, respectively $(\mathrm{P}<0.05) . \quad *$ : Significant difference between Pert2 $\mathrm{L}_{\mathrm{L}}$ and Pert3 $\mathrm{L}(\mathrm{P}<0.05)$. 


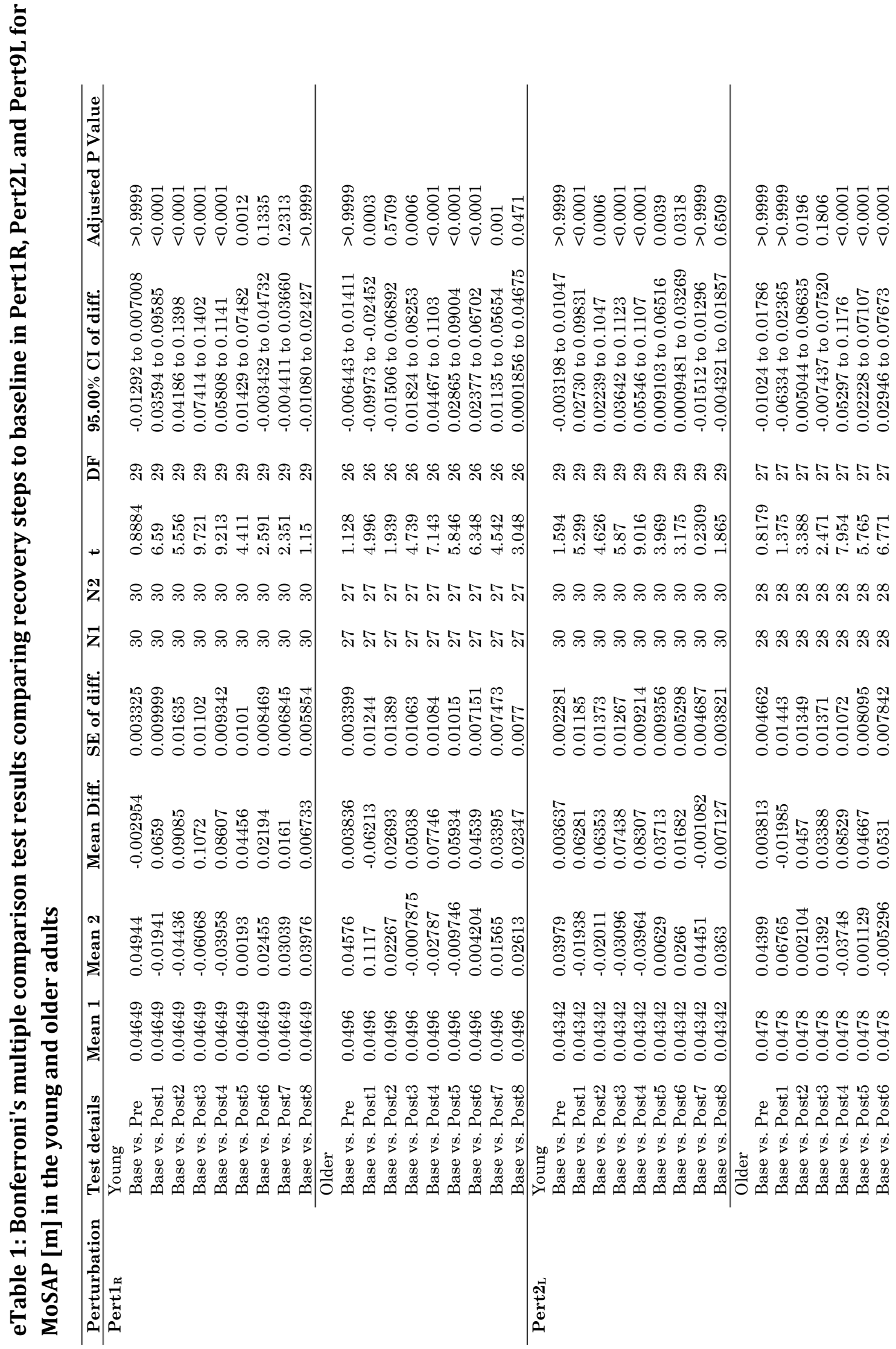




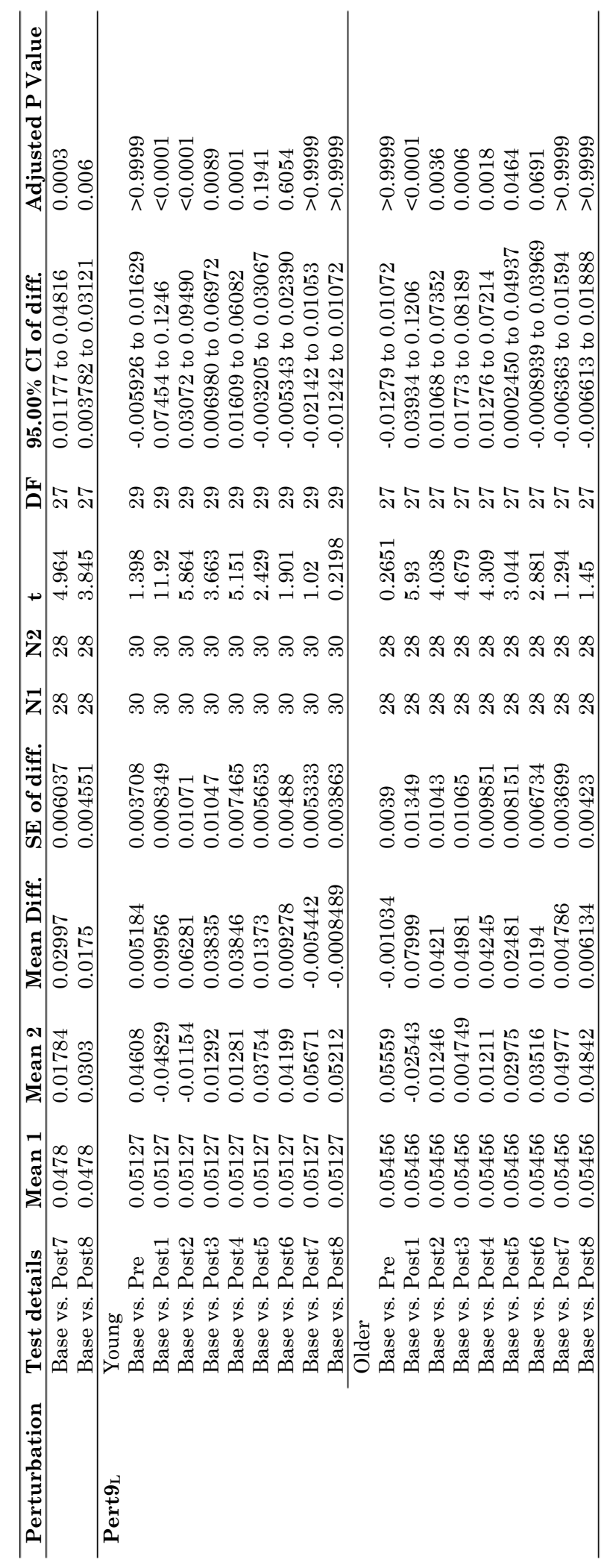




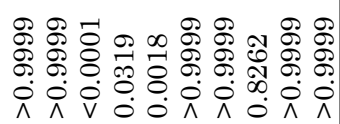

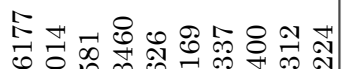

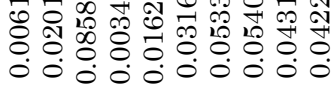

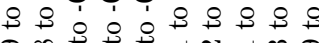

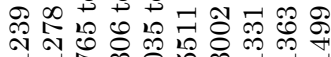

5 :

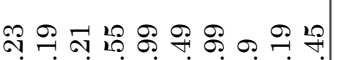

동

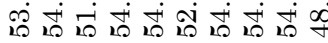

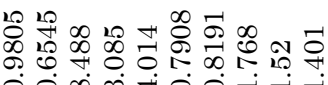

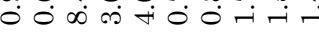

ลำลลลำลำลำลำ

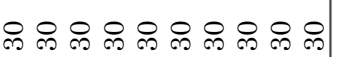

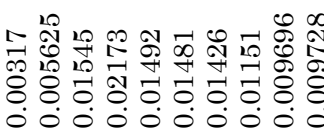

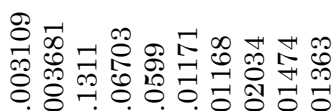

i.

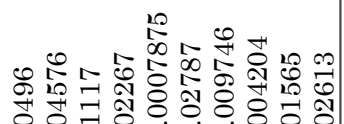

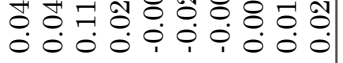

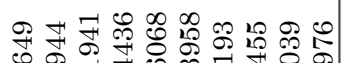

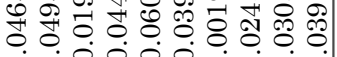

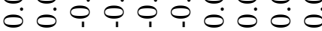

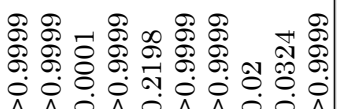

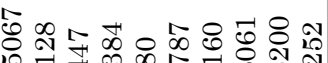
\&.

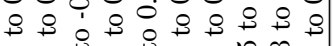

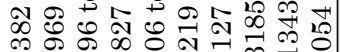
둥

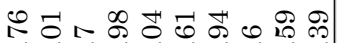

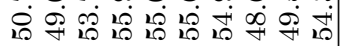

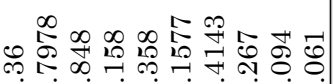

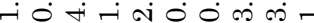

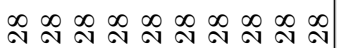

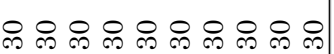

我 o.

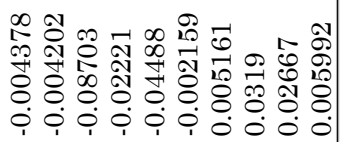

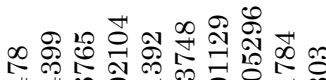

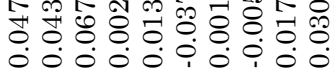

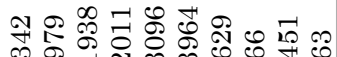

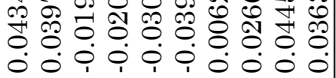
क्षेّ

H

展 i品 나요

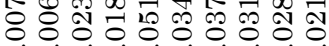
00000000.0 $+\circ$ 웅ㅇㅇㅇㅇㅇㅇㅇㅇㅛ

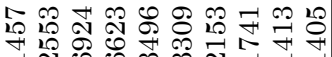

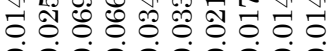
i

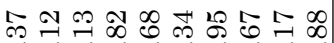

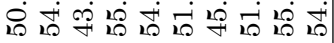

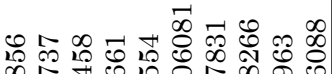

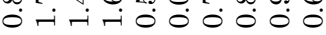

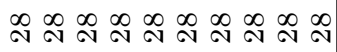

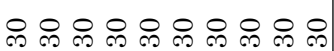

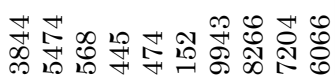
○

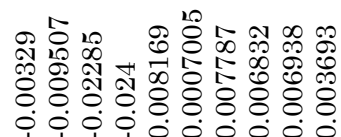

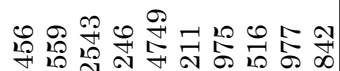

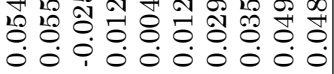
ำ 영 경

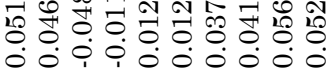
$\frac{\ddot{ \pm}}{0}$

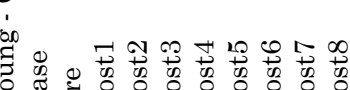




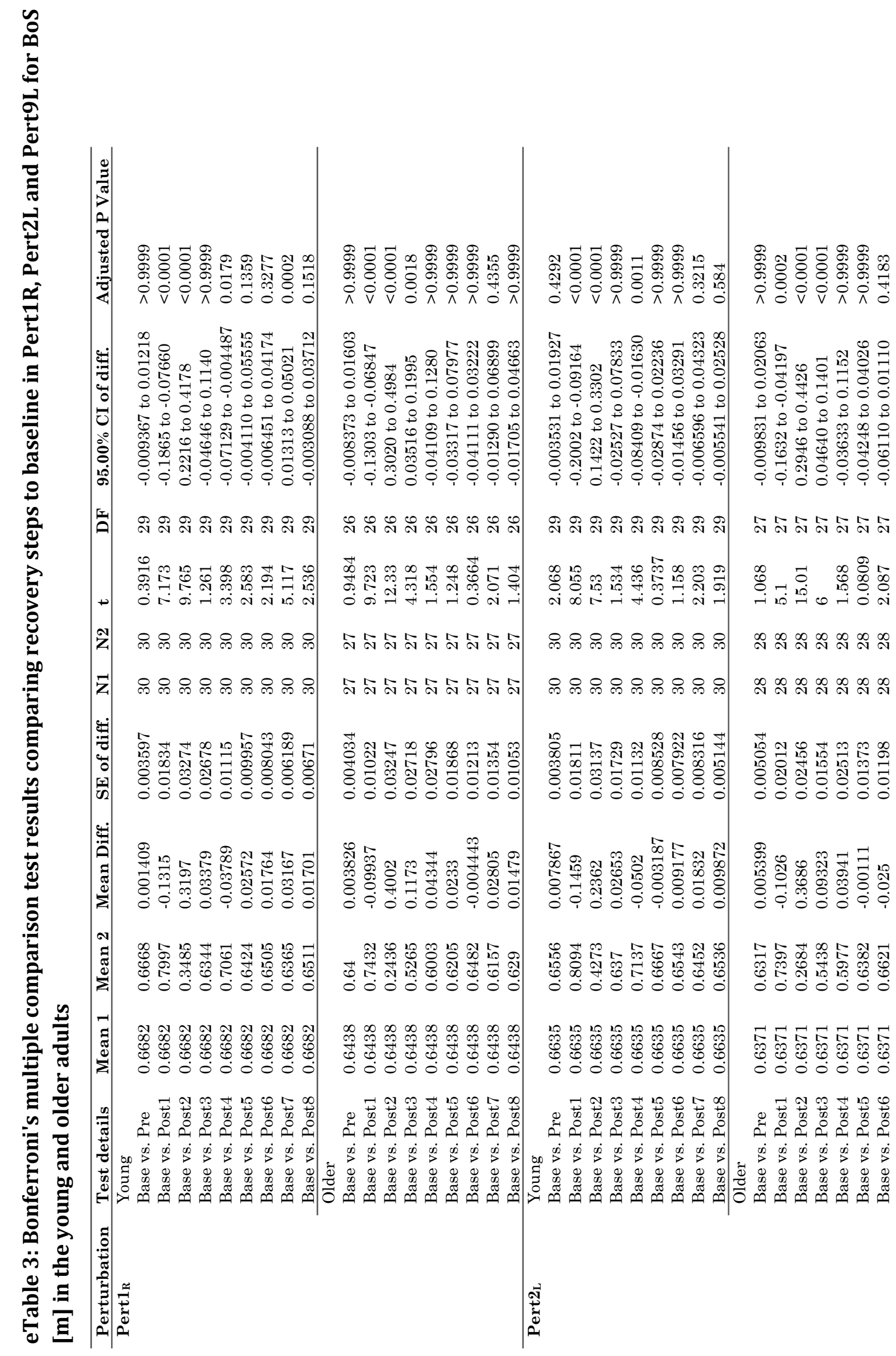




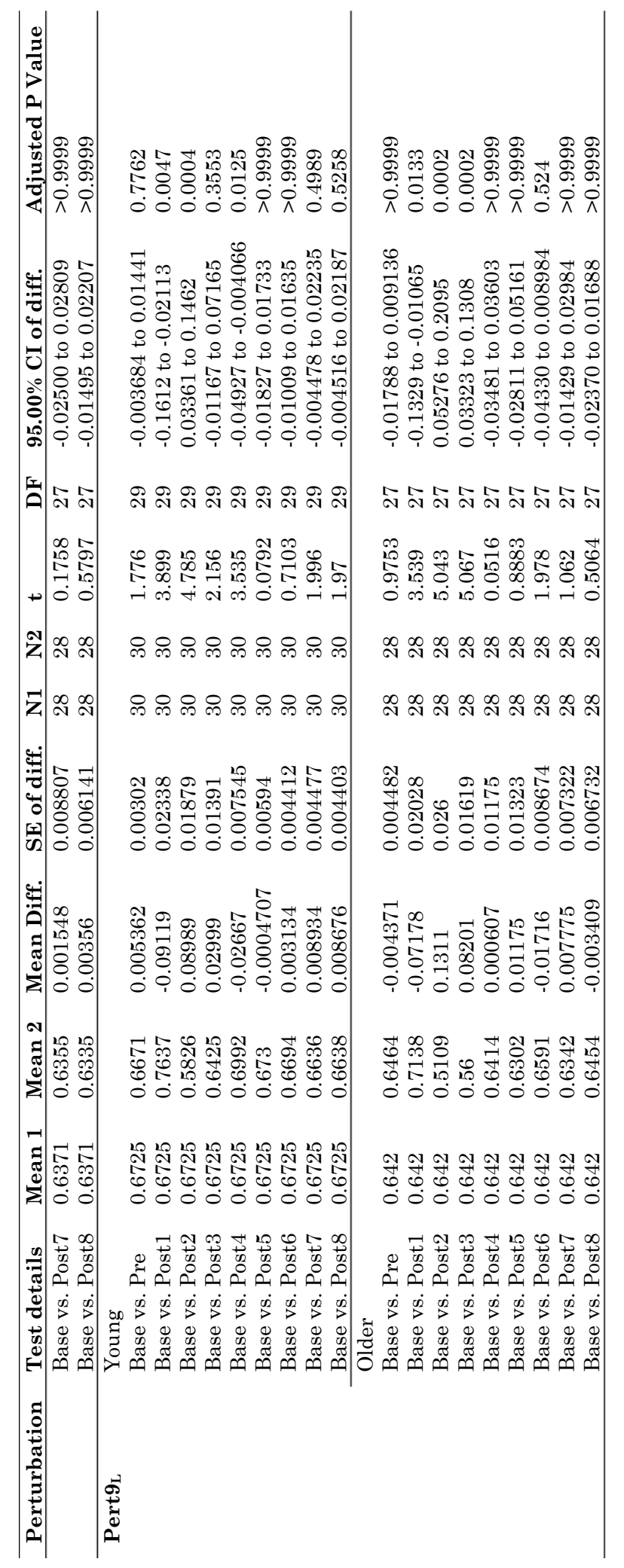




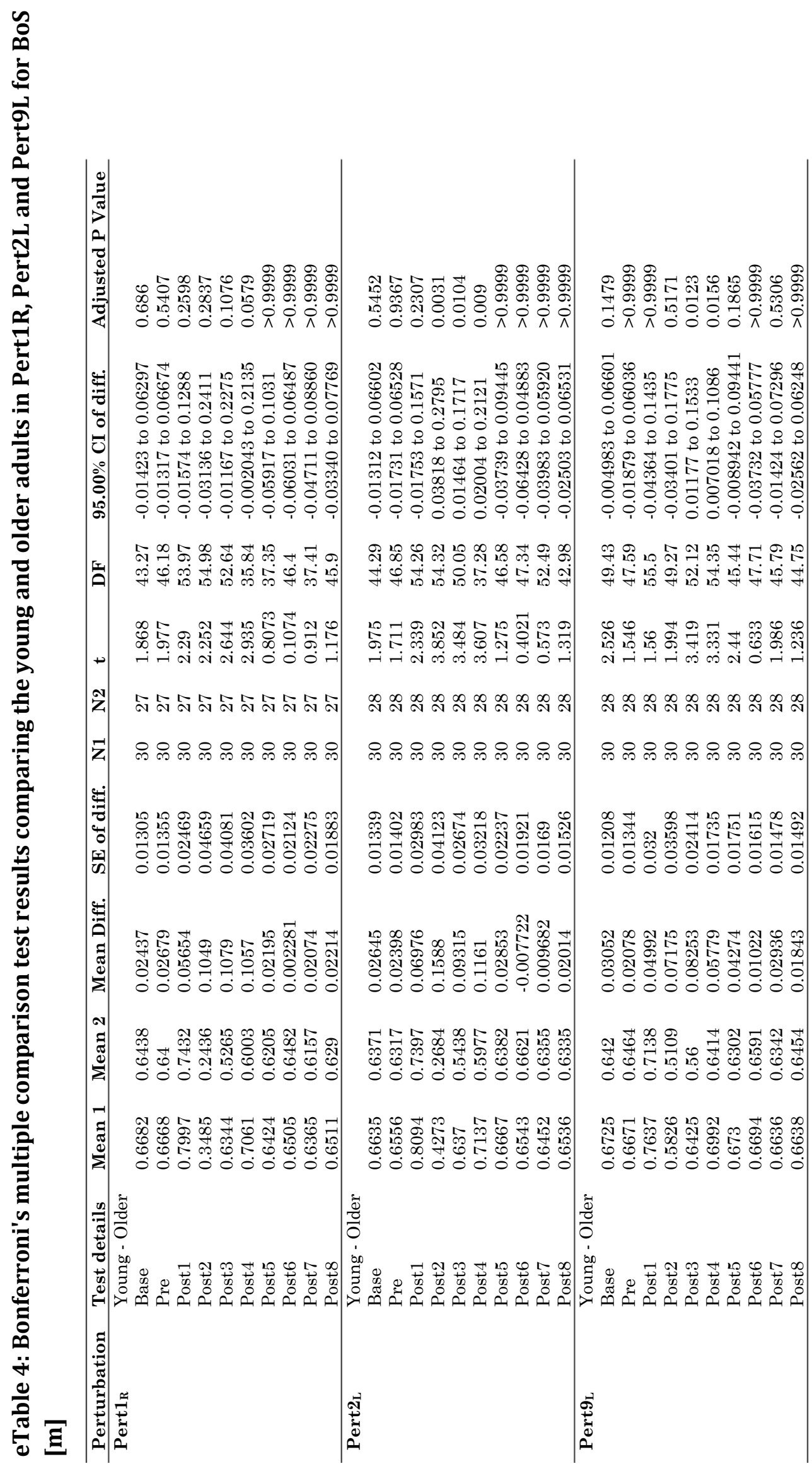




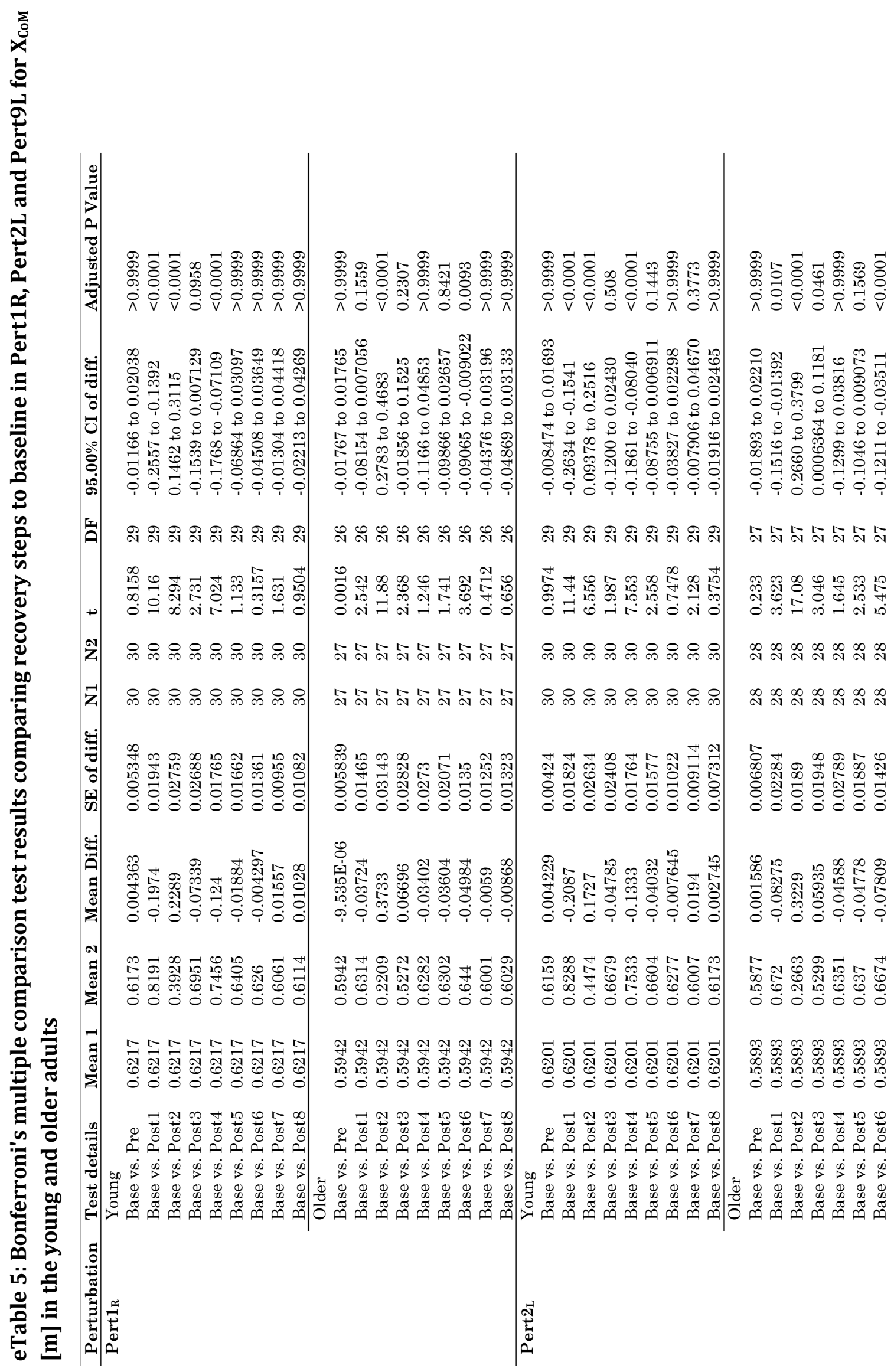




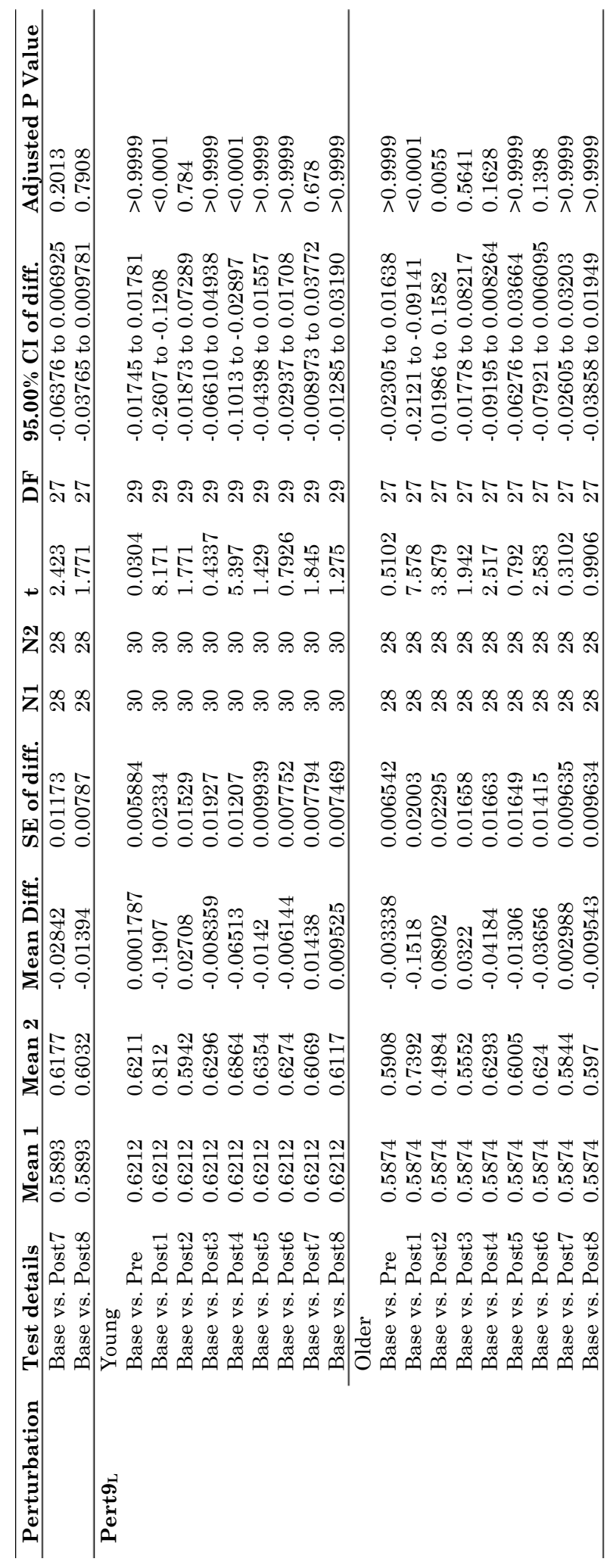




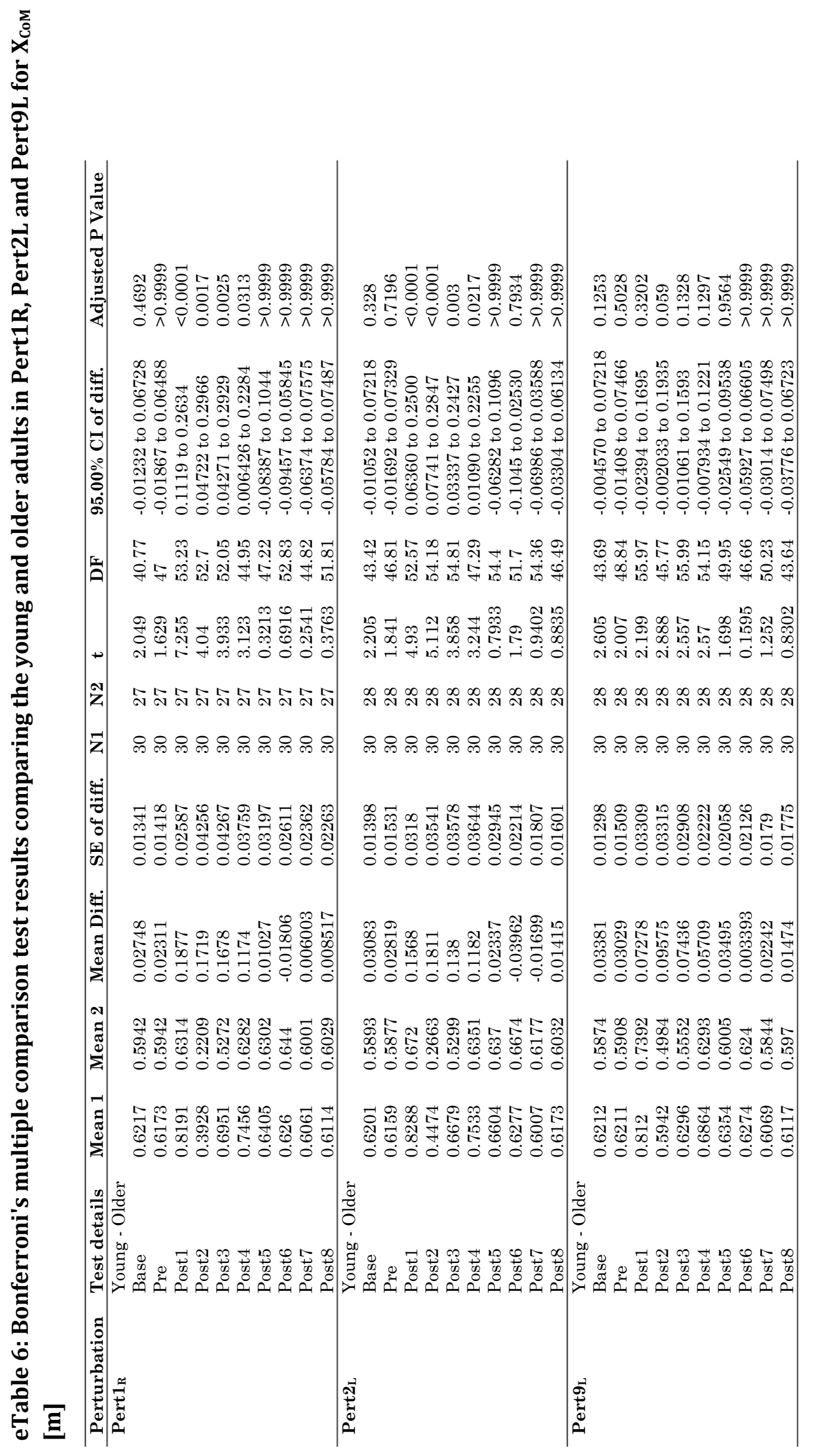




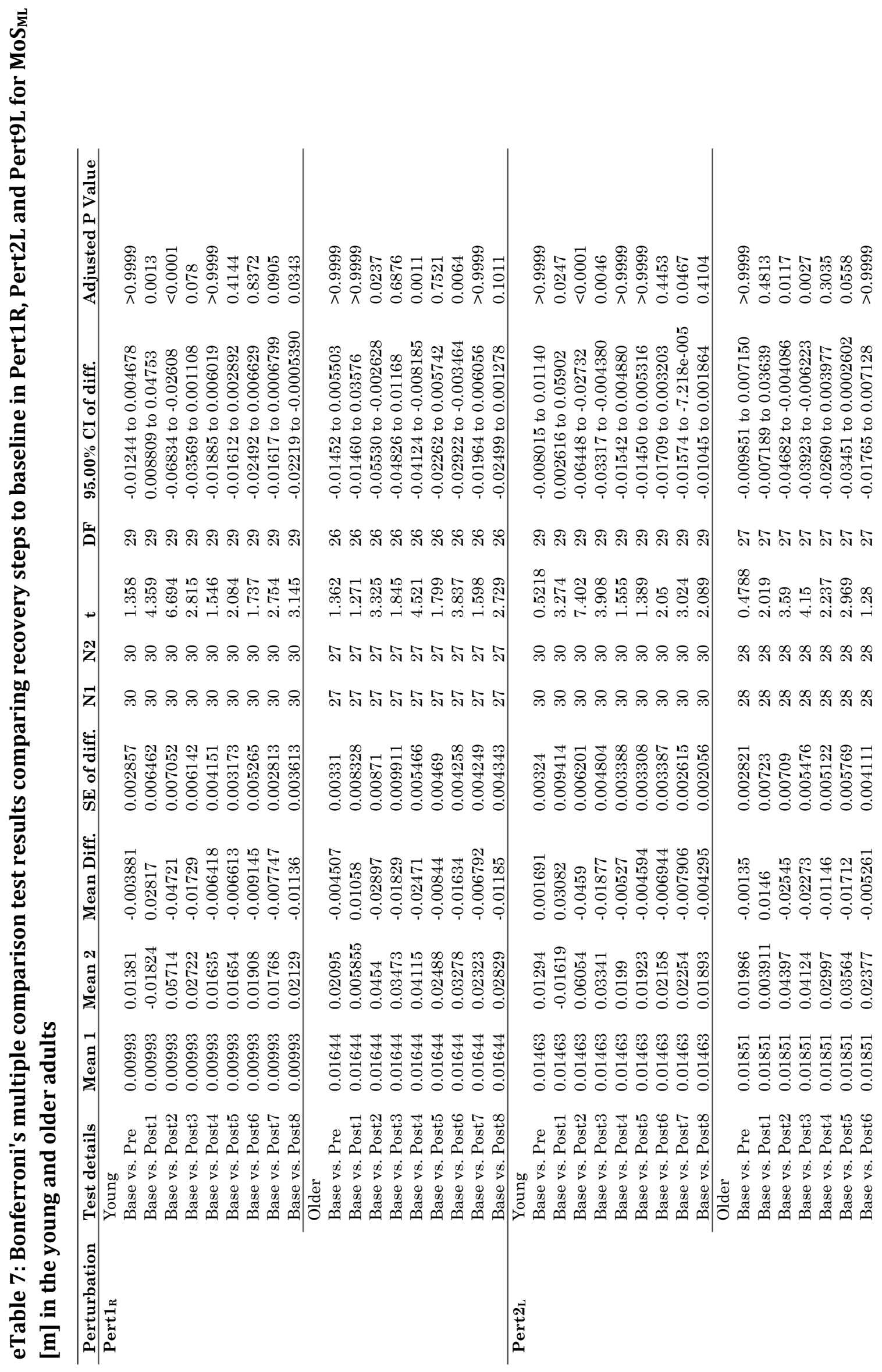




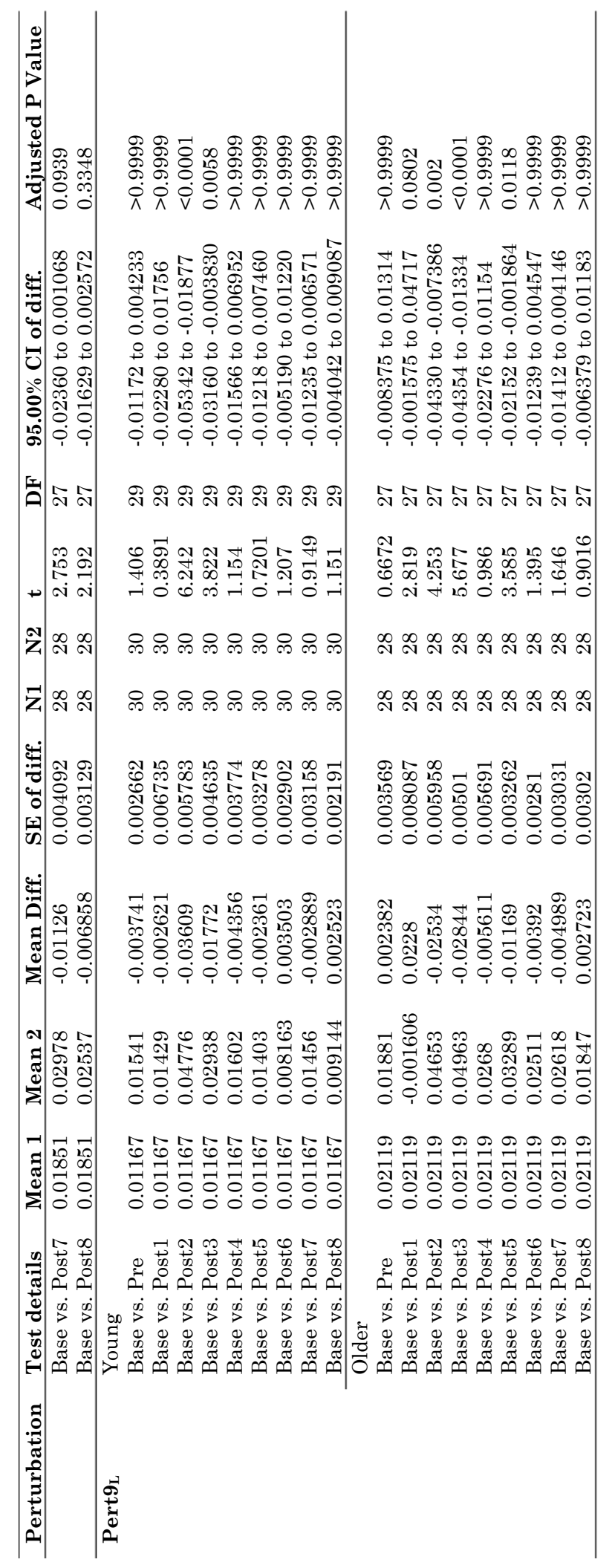




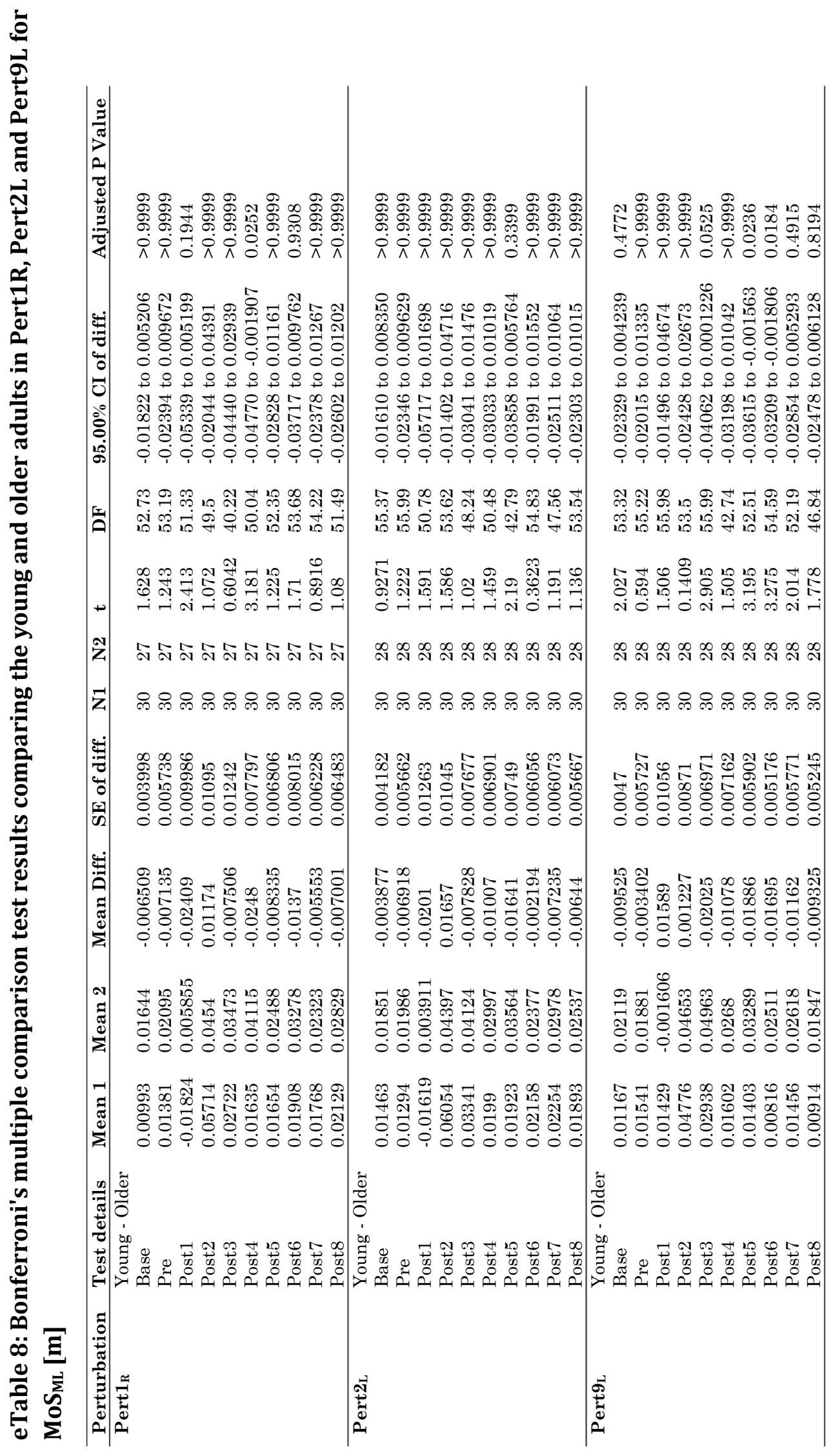




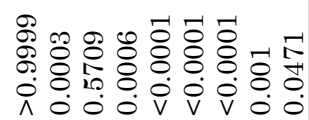

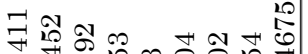

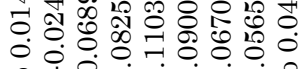

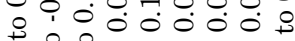

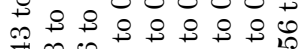

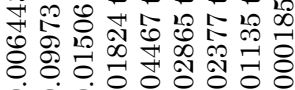

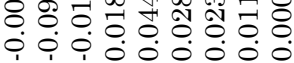

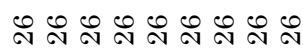

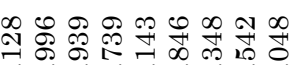
Ii

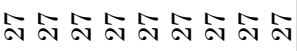

ลัลลัลัลัลัลัล

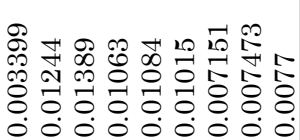

롤

ம

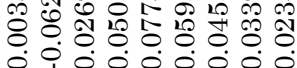

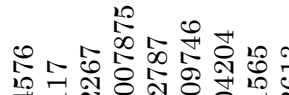

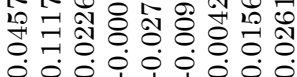

ஆ \% \& \% \%

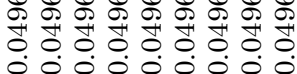

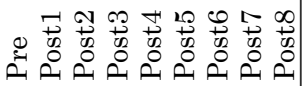

居 र

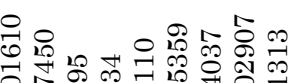
노의

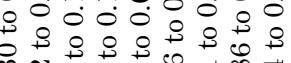

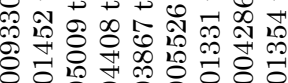

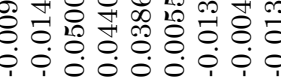
๗ బొ⿻

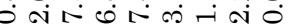
ลิลักิล ลิล ลิล

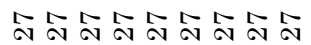

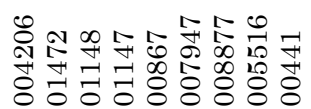

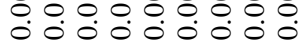

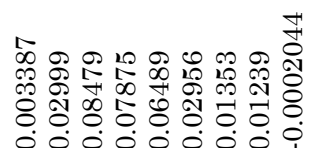

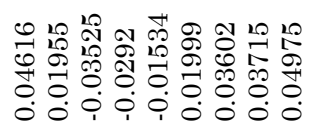

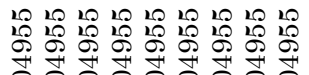

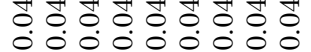

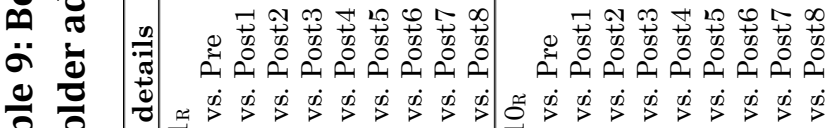
\%

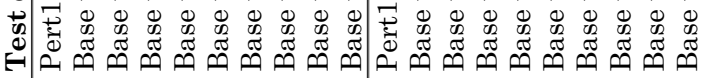

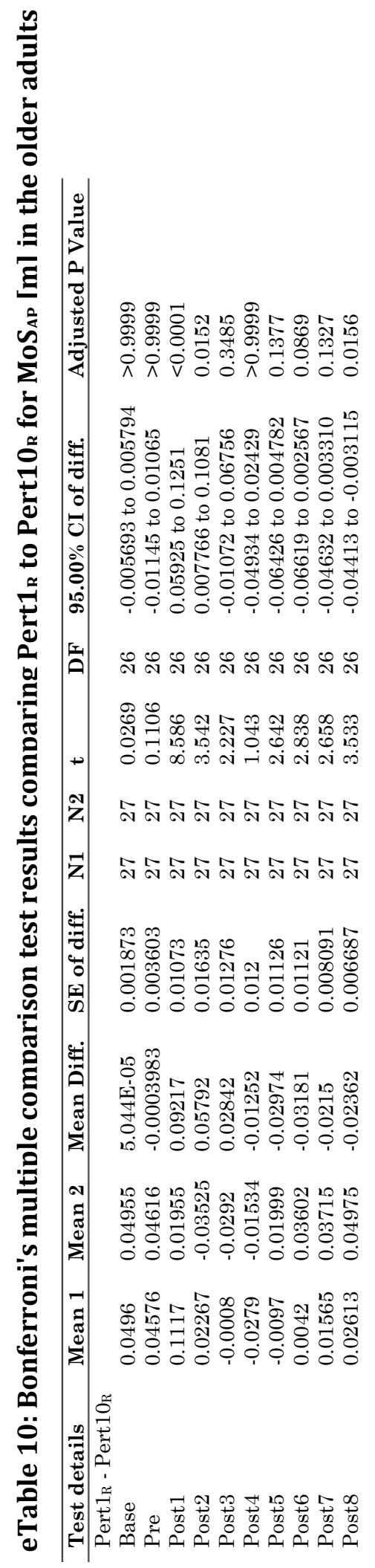



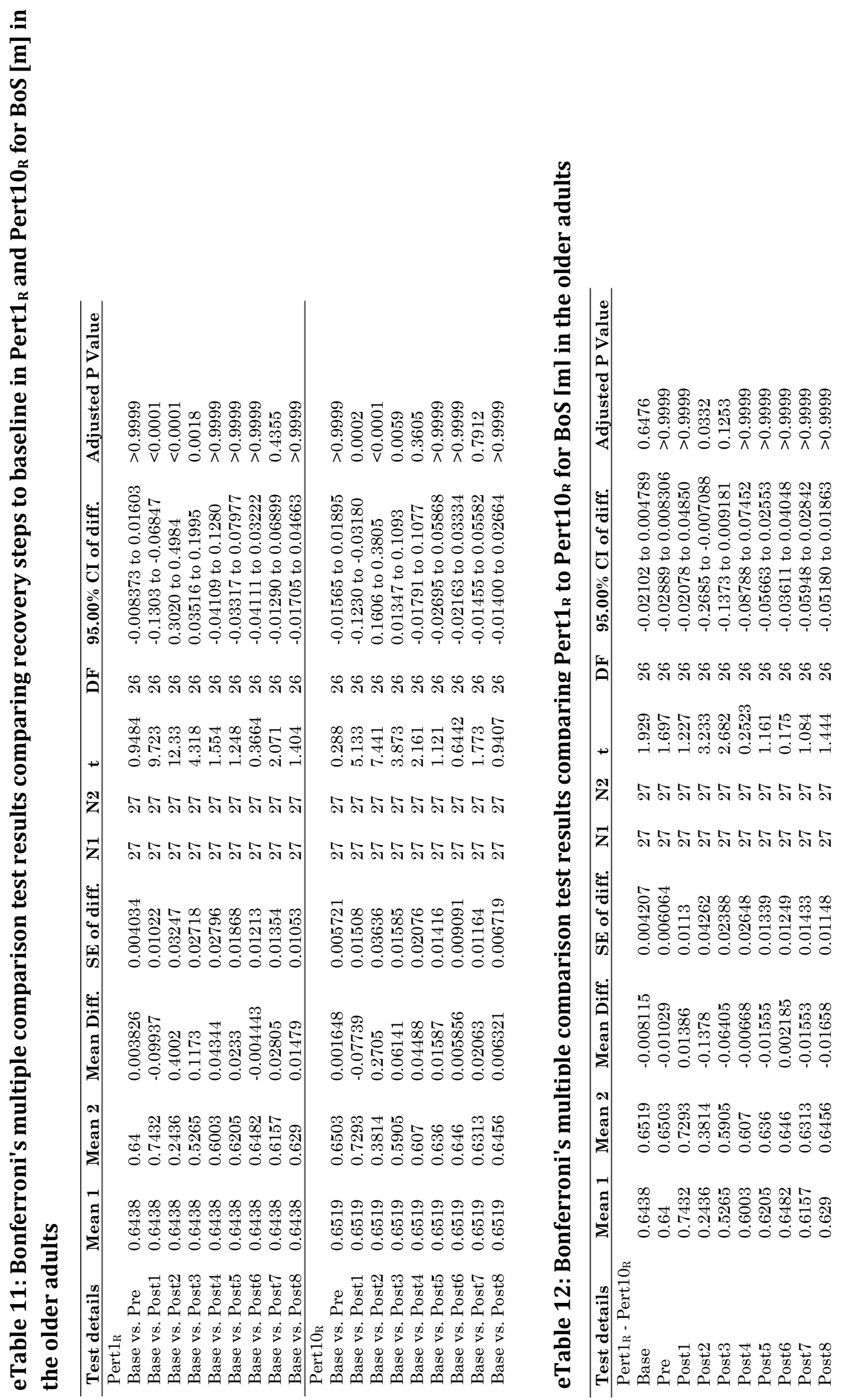

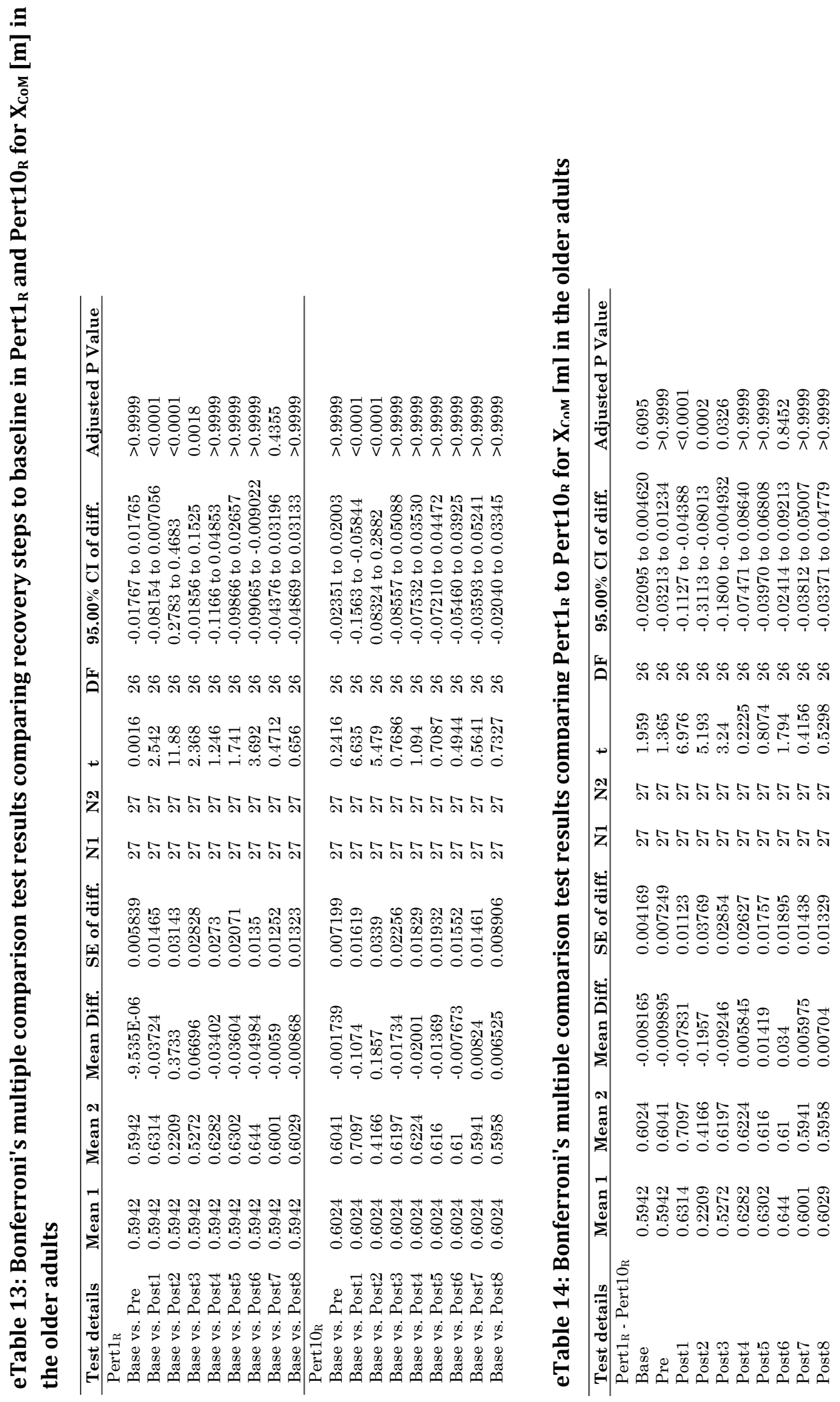

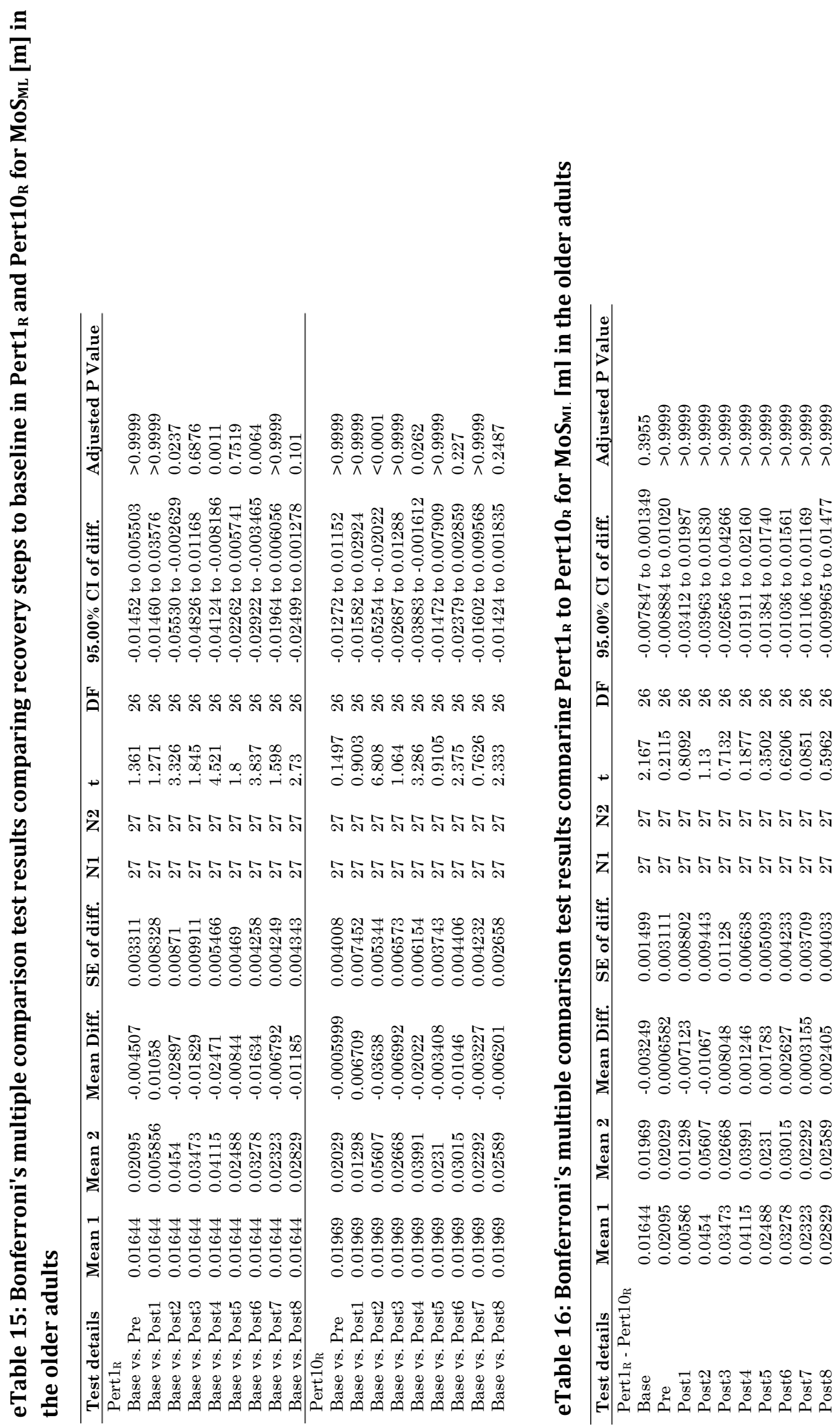

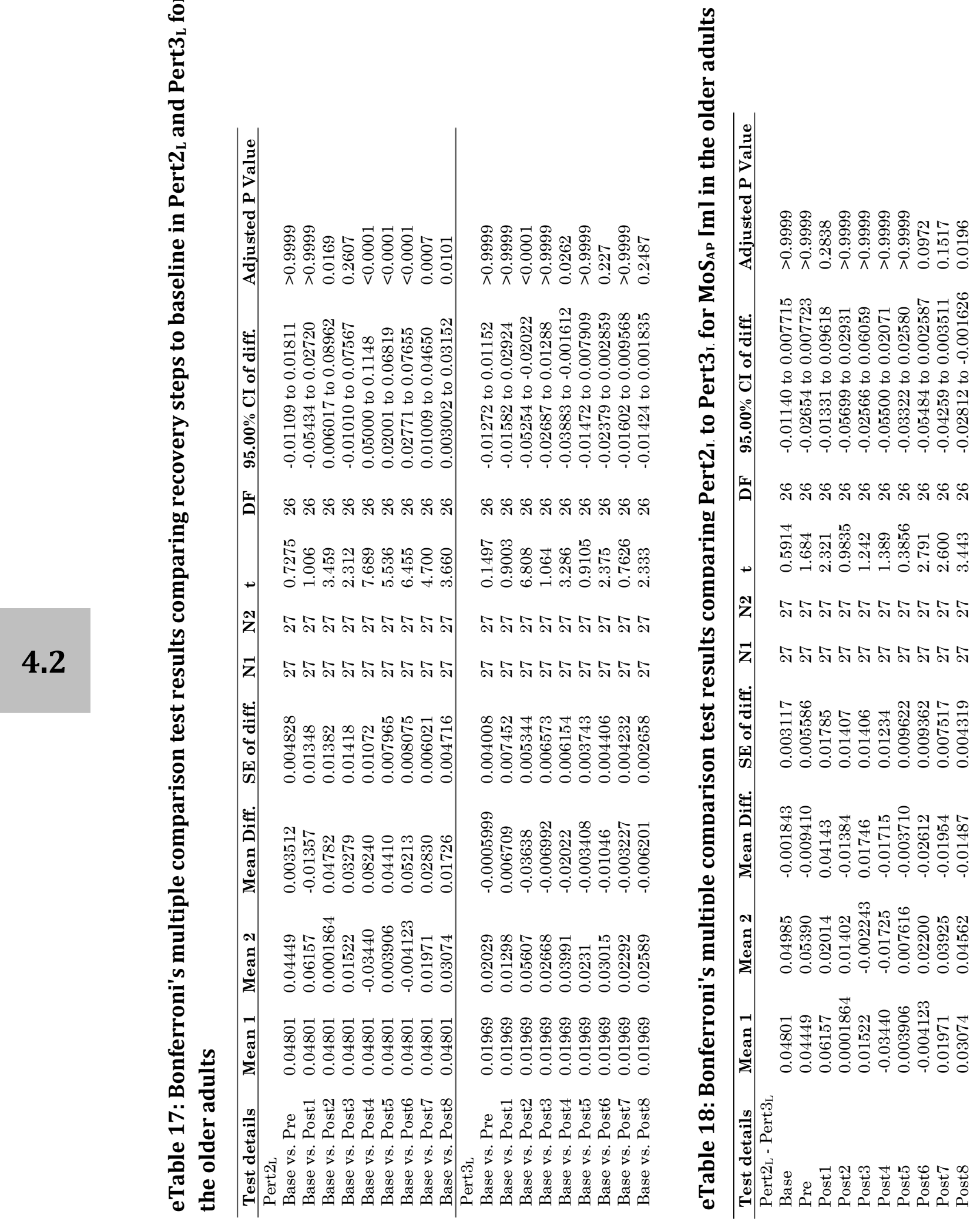


\section{eDiscussion}

Regarding our analyses of MoSML, the results did not reveal any substantial differences with age. Both age groups showed a significant increase in MoSmL in some of the first few steps post-perturbation, but there was little change in magnitude of these values with repetition. This suggests that anteroposterior gait perturbations of this nature require some mediolateral balance control but can be accommodated similarly by young and older healthy adults. It is not possible to say, based on the current work, if different magnitudes or types of anteroposterior perturbations would show a similar lack of effect with age. Sideways falls are prevalent among older adults in long-term and many falls initially directed anteriorly may lead to a lateral ground contact (Yang et al. 2016). Previous findings indicate that older age and falls history are related to lateral instability following anteroposterior perturbations to stance (McIlroy and Maki 1996; Rogers et al. 2001). However, as the current study deals with perturbations during gait, stance perturbations are obviously not directly comparable to those used in the current paradigm. A limitation that should be noted is that while our reduced kinematic model has been previously validated for the MoS $\mathrm{AP}_{\mathrm{P}}$ and its components (Süptitz et al. 2013), it was not validated for the MoSmL. Various levels of agreement have been reported in previous studies of CoM position estimates between simplified marker sets and the full-body marker sets (Havens et al. 2018; Huntley et al. 2017; Vanrenterghem et al. 2010; Yang and Pai 2014) and these differences can stem from differences in the marker model (e.g. with and without trunk), the analysed locomotion task (e.g. level walking, cutting manoeuvres), the participant groups (e.g. healthy young adults, stroke patients) and the gait velocity and experimental setup (e.g. overground and treadmill walking). Therefore, we would caution against drawing firm conclusions regarding our MoSmL results. 


\section{Part Five: \\ Improving the Reactive Stability of Older Adults}

"Preserved locomotor adaptability is the basis for the design and application of effective intervention strategies targeting fall prevention."

- Bohm et al. (2015) 


\section{1:}

\section{Perturbation-based balance training for falls reduction among older adults: current evidence and implications for clinical practice}

Marissa H. G. Gerards ${ }^{\dagger}$, Christopher McCrum $\dagger$, Avril Mansfield, Kenneth Meijer

†Joint first authors

Published as:

GERARDS, M. H. G., MCCRUM, C., MANSFIELD, A. \& MEIJER, K. 2017. Perturbation-based balance training for falls reduction among older adults: Current evidence and implications for clinical practice. Geriatr Gerontol Int, 17, 2294-2303. doi: 10.1111/ggi.13082

(C) The Authors 2017. This work is licensed under the Creative Commons Attribution-NonCommercial 4.0 International licence (CC BY-NC 4.0). https://creativecommons.org/licenses/by-nc/4.0/ 


\begin{abstract}
Falls are a leading cause of injury, hospitalization and even death among older adults. While various strength and balance exercise interventions have shown moderate reductions in falls incidence among healthy older adults, no significant falls incidence improvements have been consistently seen in frail older adults or in patient groups with an increased falls risk (e.g. people with Parkinson disease and stroke). This may be due to a lack of task specificity of previous exercise interventions to the recovery actions needed to prevent a fall. Perturbation-based balance training (PBT) is an emerging task-specific intervention that aims to improve reactive balance control following destabilizing perturbations in a safe and controlled environment. While early studies were conducted predominantly in research laboratory settings, work in clinical settings with various patient groups has been proliferating. A systematic search of recent PBT studies revealed significant reduction of falls incidence among healthy older adults and certain patient groups (e.g. people with Parkinson's disease and stroke), with clinically relevant reductions in frail older adults. The most practical methods in clinical settings may be treadmill-based systems and therapist applied perturbations and PBT that incorporates multiple perturbation types and directions may be of most benefit. While more controlled studies with long-term follow-up periods are needed to better elucidate the effects of PBT on falls incidence, PBT appears to be a feasible and effective approach to falls reduction among older adults in clinical settings.
\end{abstract}




\section{Introduction}

Falls and fall-related injuries represent a global public health concern for our ageing societies. Approximately 30\% of people over 60 years old experience a fall in a given year (Tinetti et al., 1988, Talbot et al., 2005), with older age and frailty independently increasing falls risk (Talbot et al., 2005, Kojima et al., 2015, Gale et al., 2016). Older adults with neurological disorders such as stroke and Parkinson's disease are at an even higher risk of falling (Homann et al., 2013). Falls are a leading cause of injury, hospitalization and even death among older adults (Tinetti et al., 1988, Terroso et al., 2013); therefore, evidence-based interventions for reducing falls and fall related injuries in older populations are of great importance.

Moderate reductions in falls risk (approximately 15-20\%) have been seen in healthy older adults following exercise interventions including combinations of strength, balance and aerobic exercises (Sherrington et al., 2008, Gillespie et al., 2012). However, there is mixed evidence for whether such exercise interventions result in a significant reduction in falls incidence in frail, older adults (Faber et al., 2006, Fairhall et al., 2014, de Labra et al., 2015). Importantly, there is limited evidence for falls risk reduction after such strength and balance exercise interventions alone in older adults with Parkinson's disease (Allen et al., 2010, Canning et al., 2015) or after a stroke (Verheyden et al., 2013). One potential reason for the inconsistency or lack of effectiveness of such general exercise interventions for falls reduction is the lack of task specificity to the recovery actions needed to prevent a fall (Oddsson et al., 2007, Grabiner et al., 2014). In order to recover balance following a postural disturbance, change-in-support movements (e.g. by taking compensatory steps or by grasping nearby objects for support) and counter rotations of body segments can be executed (Maki and McIlroy, 2005, Hof, 2007). Training that targets such balance recovery mechanisms may be more effective than general exercise (Oddsson et al., 2007, Mansfield et al., 2010, Grabiner et al., 2014, Carty et al., 2015).

The importance of task specific training has led to increasing interest in a new approach called perturbation-based balance training (PBT) (Pai and Bhatt, 2007, Maki et al., 2008). PBT is a task-specific intervention that aims to improve reactive balance control (i.e. rapid reactions to instability) following destabilizing perturbations in a safe and controlled environment. Participants are exposed to unexpected balance perturbations (e.g. treadmill accelerations, waist pulls, cable-based trips, nudge from a therapist etc.; see Fig. 1 for examples) during tasks of daily living, such as standing, walking or rising from a chair (Mansfield et al., 2010, Pai et al., 2010). The perturbations during PBT are unannounced in order to mimic the accidental and unexpected nature of falls in daily life (Pai and Bhatt, 2007) and ensure that the task-specific approach of PBT is in concordance with the "specificity of learning" hypothesis (Bachman, 2013).

Despite the diminished reactive gait stability seen in older adults in response to a novel perturbation compared to young adults (Süptitz et al., 2013), reactive locomotor adaptation potential (the ability to adapt and improve reactive gait adjustments in a feedback-driven manner) does not appear to decline with age (Bohm et al., 2015, Dijkstra et al., 2015), nor does it appear to be specific to one mode (stance, sit-to-stand or gait) of locomotion (McCrum et al., 2016b). By capitalizing on older adults' potential for 


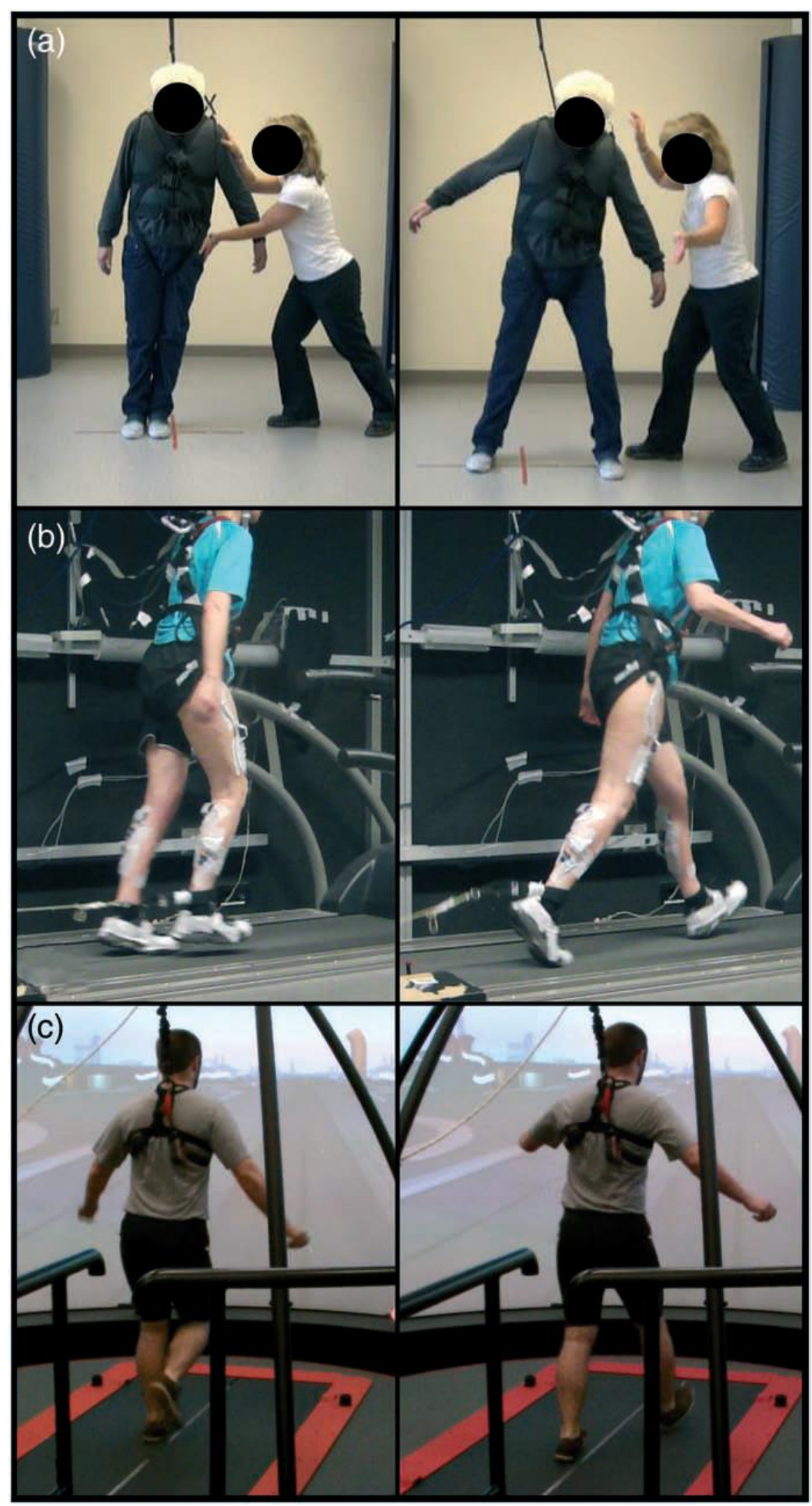

Fig. 1: Examples of different types of perturbations used in clinical and research settings. (a) A therapist-applied lean-and-release perturbation in the mediolateral direction. (b) A cable trip perturbation on a standard treadmill causing a forward loss of balance. (c) A treadmill belt acceleration perturbation using the Computer Assisted Rehabilitation Environment (Motekforce Link, Amsterdam, The Netherlands) causing a forward loss of balance. 
improvement by providing sufficient and specific stimuli (i.e. PBT), the reactive balance control of older adults could be improved, which may reduce falls risk. One recent metaanalysis of randomized controlled trials using PBT indeed reported significantly lower falls incidence in PBT groups following the interventions (Mansfield et al., 2015b), with a second meta-analysis combining studies of PBT with voluntary stepping interventions also reporting reduced falls incidence (Okubo et al., 2017). However, despite this evidence, it is important to consider whether such training is effective and feasible in clinical settings, or whether such benefits are only seen in highly controlled laboratory settings, information that is not yet explored in detail in the literature. Therefore, in this review, we systematically searched the literature for PBT studies with older adults in order to: a) examine the characteristics of PBT studies conducted to date with older adults that assessed prospective falls incidence; and b) using this evidence from the literature, present and discuss a number of considerations for applying PBT in clinical settings, such as the perturbation characteristics (type, direction, magnitude etc.) and the training program (frequency, volume), that could affect the feasibility and effectiveness of PBT for falls reduction among older adults in clinical settings.

\section{Methods}

A systematic literature search with search terms relating to perturbations, training, falls and age with date of publication set at 2002 or later was conducted in PubMed and Web of Science databases (see Appendix S1 for the full search strategy). Studies were selected for inclusion if they conducted PBT with older adults (mean age of 60 years or older), reported post-intervention falls data and if a control group was included. Studies that conducted PBT but that did not provide specific details on the intervention were excluded from the main synthesis. The final search was conducted on January $9^{\text {th }} 2017$. Additionally, reference lists of the discovered articles, previous reviews and other articles known to the authors were checked. Studies with healthy older adults, high risk or frail older adults, as well as older adults with neurological disorders that met the above criteria were considered in the current review.

\section{Results and Discussion}

The complete search and inclusion process can be seen in Fig. 2. The search yielded 802 records and four articles were identified through other sources. After removing duplicates, 672 titles were screened. The title screening excluded 489 records, after which the remaining 183 abstracts were assessed for inclusion. 32 full texts were then assessed and eight articles met all inclusion criteria. The reasons for exclusion at the full text screening stage can be found in Fig. 2. A summary of all included articles can be found in Table 1. 


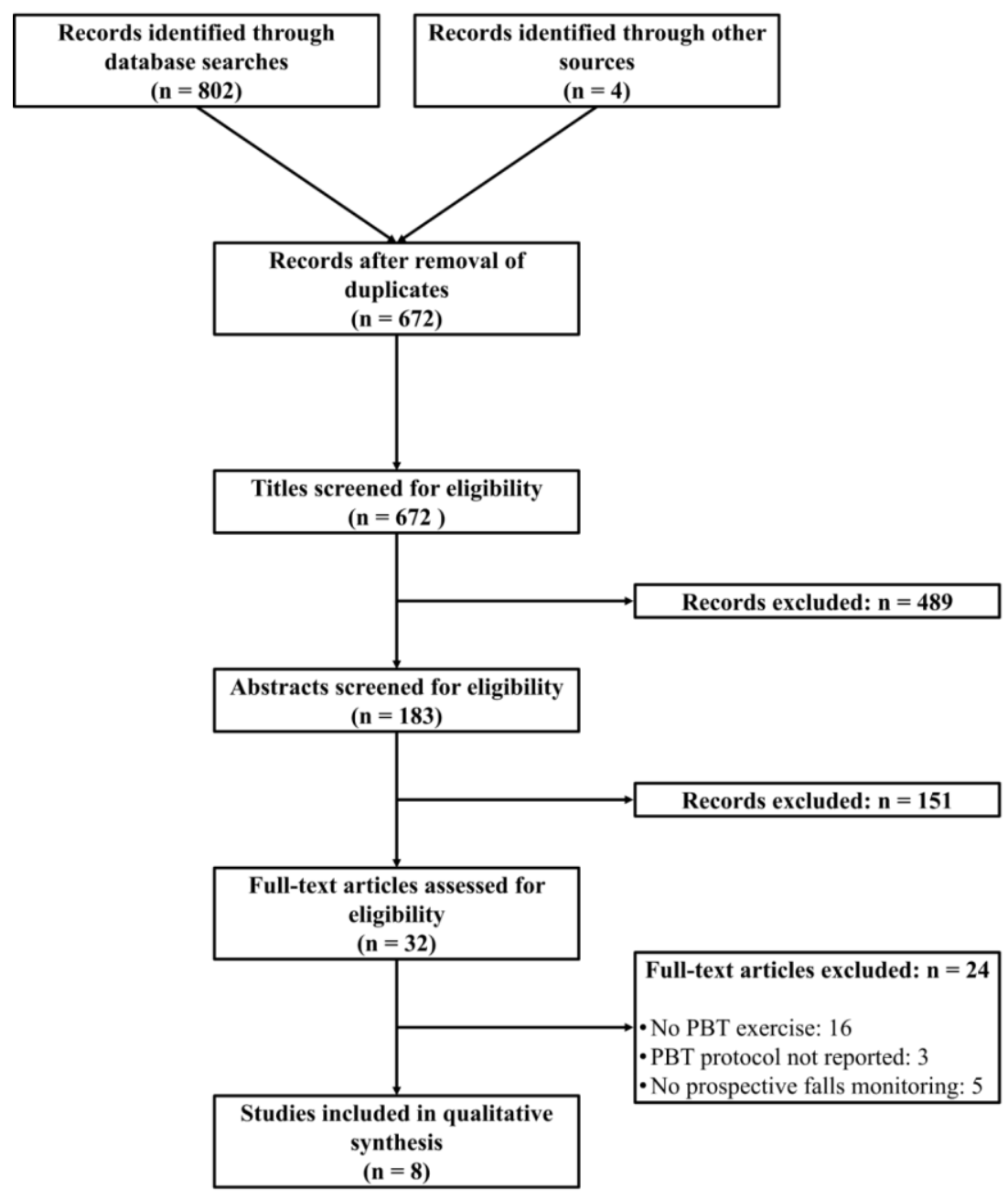

Fig. 2: Flowchart of systematic search and article inclusion and exclusion process

\section{PBT and Falls Reduction}

Three PBT studies have been conducted with healthy, community-dwelling older adults that prospectively monitored falls and included a control group (Mansfield et al., 2010, Rosenblatt et al., 2013, Pai et al., 2014a). Rosenblatt et al. (2013) examined the effects of PBT on the falls incidence over 1 year of 82 community-dwelling women (mean 65.4 (SD 7.8) years old) who received 2 weeks (4 1-hour sessions) of PBT (treadmill accelerations) compared to a control group. During the 1 year follow-up, the control group $(\mathrm{n}=80)$ experienced 31 likely preventable trip-related falls (i.e. compensatory stepping was possible), compared to a significantly lower 17 likely preventable trip-related falls in the intervention group (Rosenblatt et al., 2013). Pai et al. (2014a) also found a significant reduction in falls incidence after PBT in their study (67 community-dwelling older adults completed the PBT and 1 year follow-up; 72 (5.5) years old). Participants were exposed to either just 1 slip or a single PBT session of 24 unannounced slips. During the follow-up period of 12 months, the intervention group had a $50 \%$ decrease $(34 \%$ to $15 \%, P<0.05)$ in falls incidence, while no change in falls incidence was seen in the control group, who were 


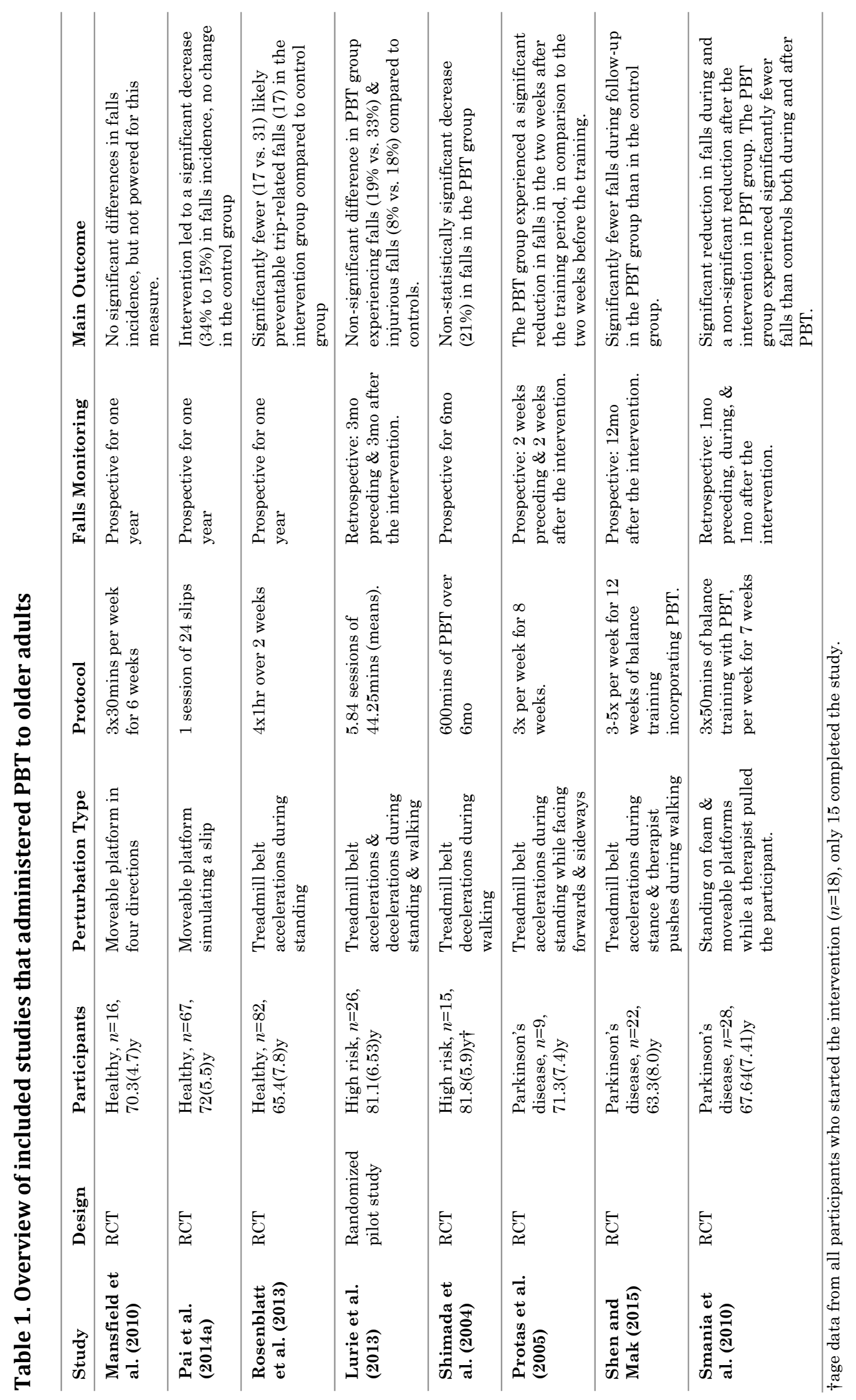


2.3 times more likely to fall than those in the intervention group in the 12 month followup period (Pai et al., 2014a). Finally, Mansfield et al. (2010) examined the effects of PBT over 6 weeks using a moveable platform during stance to train stepping and grasping reactions in older adults and found beneficial effects on balance recovery responses to labbased perturbations. The original publication did not report falls data but prospective falls data were recorded (reported in Mansfield et al. (2015b)). These data did not reveal significant reductions in falls incidence; however, the study was not powered for this outcome measure.

Two studies have examined the effects of PBT on falls incidence among frail or high risk older adults (Shimada et al., 2004, Lurie et al., 2013). Shimada et al. (2004) examined the effects of adding 600 minutes of PBT to an existing 6-month physical exercise intervention consisting of balance, strength, endurance and pain relieving exercises on falls incidence in a group of 15 long-term care facility residents and outpatients at a high risk of falling ( 4 were patients with Parkinson's disease, 4 were patients who had strokes, 8 were patients with knee osteoarthritis, the remaining 16 had no specific diagnosis). Participants were randomized to the normal exercise intervention or PBT plus the normal intervention. During the 6 -month follow-up period, the number of falls was $21 \%$ lower in the intervention group than in the control group which despite being clinically relevant, was not statistically significantly different $(P=0.384)$ to the control group (Shimada et al., 2004). Lurie et al. (2013) found similar results in 31 older adults who were referred to a physiotherapist for gait and balance training. They compared the results of PBT in addition to regular physiotherapy to a control group that received regular physiotherapy consisting of strength, mobility and balance exercises. During the 3 month follow-up period, fewer participants in the intervention group experienced falls $(19.23 \%$ vs. $33.33 \%$, $P=0.227)$ and injurious falls $(7.69 \%$ vs. $18.18 \%, P=0.243)$ in comparison to the control group (Lurie et al., 2013). As with Shimada et al. (2004) these results were not statistically significant, despite the clinically relevant differences. However, this was a pilot study and was not powered to detect differences in falls incidence. Additionally, the 3 month follow up period may have been too short to detect significant differences.

Three studies have examined the effects of PBT on the incidence of falls in daily life among older adults with Parkinson's disease (Protas et al., 2005, Smania et al., 2010, Shen and Mak, 2015). We do not discuss the above-detailed study of Shimada et al. (2004) here, as only a proportion of the participants had a neurological disorder. Protas et al. (2005) investigated the effects of 8 weeks PBT, in combination with gait training, in 9 men with mild to moderate idiopathic Parkinson's disease and showed a significant reduction of falls in the two weeks after the training period, in comparison to the two weeks before the training. Smania et al. (2010) conducted a similar study in 28 older adults with moderate to severe Parkinson's disease. 7 weeks balance training incorporating PBT was compared with general physical exercise for effects on falls incidence during, and for 1 month following the intervention (Smania et al., 2010). PBT led to a significant reduction in falls during and a non-significant reduction after the intervention compared to the month before (Smania et al., 2010). In comparison to the control group, the PBT group experienced significantly fewer falls both during and after the intervention (Smania et al., 2010). Finally, Shen and Mak (2015) reported significantly fewer falls in older adults with 
mild-to-moderate Parkinson's disease during a 15-month follow-up after 3 months balance training including PBT, compared to participants who had completed strength training. While the results of these studies suggest a beneficial effect of PBT on falls risk in Parkinson's disease, all of the interventions had multiple components, only one of which was PBT and therefore, the exact effect of PBT is difficult to determine. That being said, one recent study demonstrated that people with Parkinson's disease can adapt their reactive dynamic stability control following perturbations to stance and retain motor adaptations to a similar degree as healthy older adults over 24 hours (Peterson et al., 2016). This suggests that reactive adaptation may not be completely inhibited in Parkinson's disease, which is promising for the clinical implementation of PBT in this patient group.

There has been less research conducted on PBT in other patient populations. One study of the effects of PBT on falls post-training in people with chronic stroke is currently underway (Mansfield et al., 2015a). Preliminary results from another non-randomized study including individuals with sub-acute stroke shows a trend for reduced falls in daily life following PBT (Mansfield et al., 2016). In both of the previous stroke studies, a physiotherapist applied perturbations via pushes and nudges. One other previous study in people with chronic stroke incorporated similar PBT exercises into an agility-based training program (Marigold et al., 2005). Reduced number of falls during lab-based platform perturbations were observed following PBT, but no differences were seen in daily life falls incidence (Marigold et al., 2005), probably due to the study not being powered for this outcome measure. Despite these promising results, more research is needed to determine the effectiveness and feasibility of PBT for falls reduction in patient groups with an increased falls risk.

\section{Implementing PBT in Clinical Practice}

In this section, we discuss a number of factors that should be considered when implementing PBT in clinical practice. This is done with reference to current research in both laboratory and clinical settings. The included studies in this review, as well as studies analyzing the effects of PBT on reactive compensatory stepping behavior following labbased perturbations are discussed, as the effects of PBT can be evaluated more precisely in such laboratory settings.

\section{PBT Setups in Clinical Practice}

While many methods are available for delivering unexpected perturbations, the PBT studies that have been conducted in clinical settings have generally opted for treadmillbased perturbations (Suteerawattananon et al., 2002, Shimada et al., 2004, Protas et al., 2005, Lurie et al., 2013, Shen and Mak, 2015) or therapist-applied perturbations (Marigold et al., 2005, Mansfield et al., 2015a, Mansfield et al., 2016). There are two practical advantages to treadmill-based setups: the lack of required space and relative ease of securing a fixed harness system above the treadmill. These studies have employed treadmill belt accelerations during stance or walking in order to perturb the participant's 
balance in a similar manner to a trip or a slip, and this setup appears to be feasible in clinical settings (Lurie et al., 2013). Therapist-applied perturbations could be considered the most clinically feasible type of perturbations, given the low cost and limited equipment required. These can be either internal perturbations (having the patient perform a task that causes instability) or external perturbations. External therapist-applied perturbations can include lean-and-release or pushing and pulling the participant in multiple directions (Mansfield et al., 2015a). The feasibility of PBT in clinical settings is also supported by case studies of patients with progressive supranuclear palsy (Suteerawattananon et al., 2002) and sub-acute stroke (Mansfield et al., 2011) reporting positive outcomes. Once the most feasible setup for PBT has been determined, it is important to consider how to best maximize the effects of PBT.

\section{Maximizing Long-Term Effects of PBT}

One key factor that may determine how successful PBT can be for reducing falls is the extent to which participants retain improvements in reactive balance control they have made during training over the weeks, months and years following training. In PBT studies, the long-term effects can be determined via prospective falls monitoring or perturbation recovery performance assessed in a laboratory setting. These improvements could be enhanced ability to increase the base of support by stepping, decreased reaction time to perturbations, or improved counter rotation to control the center of mass (Maki and McIlroy, 2005, Pijnappels et al., 2005c). This retention may be affected by a combination of the perturbation type and magnitude, but also the training volume. Previous studies reported that both healthy older adults and patients with Parkinson's disease, after experiencing a single session of perturbations, showed at least partial retention of reactive balance control improvements over short periods of 24 hours (Dijkstra et al., 2015, Peterson et al., 2016). Studies with healthy older adults have also demonstrated retention over longer periods of 6 (Bhatt et al., 2012, Pai et al., 2014b), 9 and 12 months (Pai et al., 2014b) in laboratory settings. Retention in compensatory step length has also been shown in patients with Parkinson's up to two months after a two week long PBT intervention (Jobges et al., 2004). Retention over such long time periods indicates that PBT provides a strong stimulus for the neuromuscular system, which may indicate that high training volumes may not be necessary to maximize retention. Bhatt et al. (2012) also investigated the effect of experiencing a single slip perturbation 3 months after a perturbation session on retention at 6 months. Their results show that such a "booster" session helped participants to retain improvements in reactive balance control (Bhatt et al., 2012). These findings are potentially important for clinical practice, as they indicate that after an initial training period, long-term retention of the benefits of PBT is possible and can be enhanced with short additional sessions.

\section{Perturbation Magnitudes}

One way to maximize the effects of PBT is to use perturbations of appropriate magnitude. In the studies discussed above, a variety of magnitudes were used, that were either fixed, 
or progressive with training. High magnitude perturbations, where participants initially require support from the harness to regain stability, appear to trigger fast and significant adaptation in recovery behavior and long-term retention of motor adaptations (Bhatt et al., 2011, Bhatt et al., 2012, Pai et al., 2014a, Pai et al., 2014b). However, studies based in clinical settings have generally employed a more progressive increase in perturbation magnitude, starting with lower magnitude perturbations and progressing based on the supervising physiotherapist's judgement (Smania et al., 2010, Lurie et al., 2013). While perturbation magnitudes that result in participants requiring support from the harness have been shown to be effective, these may not always be appropriate for specific groups, such as frail older adults or people with neurological conditions, as physical injury is possible even if the safety harness is used to prevent a fall to the floor. Additionally, high magnitude perturbations might not be tolerated by some frail individuals, which could increase withdrawal from the program. It is not yet clear how much perturbation magnitude impacts motor learning and retention. In young adults, exposure to smaller magnitude perturbations can improve stability control following larger magnitude perturbations (Patel and Bhatt, 2015). However, it has also been shown that younger adults can recover more effectively from an overground slip after high, rather than low, magnitude perturbation experience (Liu et al., 2016). Given the mixed evidence in young adults and the benefits of both approaches shown in older adults, selecting perturbation magnitudes that are safe and tolerable while still challenging for the participant appears to be a reasonable choice for clinical applications.

\section{Perturbation Directions}

Second to the perturbation magnitude, the direction of perturbation should be considered. In the studies discussed above, 2 studies applied perturbations that caused a loss of balance in the backward direction (Shimada et al., 2004, Pai et al., 2014a). Another study applied only perturbations leading to a forward loss of balance (Rosenblatt et al., 2013), while 3 studies applied perturbations in both directions (Protas et al., 2005, Lurie et al., 2013, Shen and Mak, 2015). Although the impact of perturbation direction on falls incidence or types of falls experienced is not known, there is evidence to suggest that adaptation to perturbations in one direction may not transfer and benefit reactive balance control in another direction (Dijkstra et al., 2015, Peterson et al., 2016). Perturbations in the mediolateral directions should also be considered when applying PBT in clinical settings, due to the reduced mediolateral stability seen in older adults (Maki et al., 2000). This reduced mediolateral stability can also be seen during forward compensatory stepping, where older adults often struggle to stabilize the leg and keep from falling sideways (McIlroy and Maki, 1996, Singer et al., 2016). While repetition of one single perturbation may improve certain mechanisms of balance control that can be transferred to other tasks (e.g. counter rotations or rapid stepping to enlarge the base of support), it seems reasonable to suggest that multidirectional perturbations that target several balance recovery strategies, may be the most advantageous for falls reduction in older adults. 


\section{PBT Frequency and Volume}

The optimal frequency and volume of PBT for falls reduction among older adults must be considered. While the duration of training sessions in previous studies has not always been described, most report sessions of 50 minutes to 1 hour. The frequency and training load varies to a greater extent from just single sessions to multiple sessions over a number of months. It is important for future research to determine the minimum effective dose for falls reduction in different participant and patient groups, as this would minimize the time and financial commitment needed for PBT in clinical settings. As stated above, this may depend on the magnitude of perturbations used. With high magnitude perturbations, relatively low PBT volume may be required for long-term benefits (Bhatt et al., 2011, Bhatt et al., 2012, Pai et al., 2014a, Pai et al., 2014b). With lower magnitude perturbations, which may be more feasible with frail, older adults or different patient groups, longer training periods may be needed in order to result in a significant reduction in falls incidence.

\section{Other Considerations}

Falls tend to occur in daily life during execution of movement (e.g., walking or transferring from standing to sitting), and rarely occur during quiet standing (Tinetti et al., 1988, Berg et al., 1997, Robinovitch et al., 2013). Therefore, perturbations should be applied during tasks such as walking (Shimada et al., 2004, Lurie et al., 2013), weight shifting (Mansfield et al., 2007, Mansfield et al., 2015a) and rising from a chair (Pavol et al., 2004). Falls can also occur in varied environmental circumstances that pose sensory and mechanical challenges to balance control (e.g., in the dark/dim light or in a presence of obstacles that impede stepping), and may occur when the individual is distracted. Therefore, PBT programs should consider adding sensory, environmental, and cognitive challenges during training (Pavol et al., 2004, Mansfield et al., 2015a) to help to promote generalizability of improved reactive balance control to realistic situations.

\section{Future Research Directions}

Based on the PBT studies discussed in this review, a number of methodological issues should be addressed in the future. Firstly, the intervention was not always standardized across participants, due to the individualization based on ability and physiotherapist judgement (Shimada et al., 2004, Smania et al., 2010, Lurie et al., 2013, Shen and Mak, 2015). While these studies demonstrate the feasibility of PBT in clinical practice, conclusions related to the optimal perturbation number and type are difficult to make. Secondly, the falls monitoring follow up period differed between studies, with 4 studies following participants for 6 or 12 months and 3 with a follow up period between 1 and 3 months. This makes comparisons across different interventions more difficult with regards to long-term benefits of PBT. As these interventions differed greatly and were conducted in different subject groups, it is difficult to determine the components of PBT that affect long-term retention in PBT-induced adaptations. Therefore, more controlled 
studies of PBT with long-term follow up are needed to better determine the effects of different PBT components on motor adaptation, retention, and falls.

\section{Conclusion}

PBT appears to be a feasible approach to reduce falls among older adults in clinical settings. Based on current evidence, it appears that treadmill-based systems and therapist-applied perturbations may be the most practical methods in clinical settings and PBT that incorporates multiple perturbation types and directions may be of most benefit. 


\section{Supporting Information}

\section{Appendix S1: Search Strategy}

PubMed Search on 09/01/2017:

(((((Perturb*[Title/Abstract] OR trip[Title/Abstract] OR slip*[Title/Abstract] OR dynamic balanc*[Title/Abstract])) AND (train*[Title/Abstract] OR exercis*[Title/Abstract] OR rehabilitation[Title/Abstract])) AND falls[Title/Abstract]) AND (Age[Title/Abstract] OR aged[Title/Abstract] OR elderly[Title/Abstract] OR older[Title/Abstract])) NOT review[Publication Type]) AND ("2002"[Date - Publication] : "3000"[Date - Publication]) Search returned: 157 results

Web of Science on 09/01/2017:

((TS=(Perturb* OR trip OR slip* OR dynamic balanc*) AND TS=(train* OR exercis* OR rehabilitation) AND TS=falls AND TS=(Age OR aged OR elderly OR older)) OR (TI=(Perturb* OR trip OR slip* OR dynamic balanc*) AND TI=(train* OR exercis* OR rehabilitation) AND TI=falls AND TI=(Age OR aged OR elderly OR older))) $A N D$ DOCUMENT TYPES: (Article)

Indexes=SCI-EXPANDED, SSCI, A\&HCI, ESCI Timespan=2002-2017

Search returned: 645 results

Records identified through database searches: 802 records 
5.1: Perturbation-based balance training for falls reduction among older adults: current evidence and implications for clinical practice

\section{1}




\section{Part Six: \\ General Discussion}

"It gets on my nerves the old 'Well science doesn't know everything'. Well science knows it doesn't know everything, otherwise, it would stop."

- Dara O’Briain

With adapted excerpts from:

MCCRUM, C., LEOW, P., EPRO, G., KÖNIG, M., MEIJER, K. \& KARAMANIDIS, K. 2018. Alterations in Leg Extensor Muscle-Tendon Unit Biomechanical Properties With Ageing and Mechanical Loading. Front Physiol, 9, 150. doi: 10.3389/fphys.2018.00150 (in section "Muscles, Tendons and Strength Training")

VAN HOOREN, B., MEIJER, K. \& MCCRUM, C. 2019. Attractive Gait Training: Applying Dynamical Systems Theory to the Improvement of Locomotor Performance Across the Lifespan. Front Physiol, 9, 1934. doi: 10.3389/fphys.2018.01934 (in section "Gait Robustness") 


\section{1: Summary of the Main Findings}

This dissertation aimed to further our understanding of how old age might affect gait stability and adaptability, with the perspective that this knowledge could improve the effectiveness and specificity of exercise-based falls reduction interventions for both healthy and clinical populations at an increased risk of falls. Taking the results of the individual chapters of this dissertation together, a number of conclusions related to this overall aim can be made.

Firstly, when assessing, comparing or attempting to improve gait stability, the walking speed used should always be carefully considered. The results of this dissertation have demonstrated that walking speed significantly affects multiple step parameters and their variability (Chapter 2.1), the stability of the body configuration (Chapter 3.2) and, potentially, the vestibular contribution to locomotor control (Chapter 2.1). Additionally, the relationship between walking speed and stability varies from person to person in both young (Chapter 4.1) and older adults (Chapter 4.2). These findings imply that the outcomes of gait stability assessment, comparisons and training will be affected by the chosen walking speed.

Secondly, older adults generally demonstrate less stability during perturbed gait, both when the difference can't be attributed to the effects of walking speed (Chapter 4.2) and during unperturbed gait in some parameters (Chapter 2.1), in comparison to young adults. However, gait remains highly adaptable in older age, to the extent that older adults can achieve similar stability values to younger adults following a certain number of repeated perturbations (Chapters 2.2 and 4.2) and can positively influence their risk of falls (Chapters 3.1 and 5.1). That being said, the triggers of gait adaptation are manifold and situation specific; within this dissertation, the rate of adaptation to a sustained perturbation was found to be slower in older adults (Chapters 2.2), whereas older adults appeared to demonstrate interlimb transfer of adaptations to sudden perturbations (Chapter 4.2) when younger adults did not (Chapter 4.1).

Finally, and related to the previous general conclusion, many unanswered questions remain regarding how to optimally stimulate long-lasting, generalisable adaptations in gait that will have the best chance of translating to a reduced risk of falls in daily life (Chapters 3.1, 4.2 and 5.1).

\section{2: Exercise-based Fall Risk Assessment and Intervention}

\subsection{1: Muscles, Tendons and Strength Training}

It has been known for some time that lower limb strength and power play important roles in the age-related changes in locomotion. In early studies, it was recognised that the ankle flexors and extensors had greater deficits with age than those of the knee (Whipple et al., 1987, Wolfson et al., 1995) and that specifically the ability to produce rapid ankle torques was reduced in older age (Thelen et al., 1996). Since then, lower plantar flexor strength, leg extension power and knee extensor muscle quality have been associated with falls history in older adults (Skelton et al., 2002, Cattagni et al., 2014, Gadelha et al., 2018b) and knee extensor strength and muscle quality have been reported 
to distinguish future fallers from non-fallers (Gadelha et al., 2018a). Laboratory studies have also revealed associations between lower limb muscle strength and balance recovery following lean-and-release (Grabiner et al., 2005, Karamanidis and Arampatzis, 2007, Karamanidis et al., 2008, Carty et al., 2012a, Carty et al., 2012b), trip (Pavol et al., 2002a, Pijnappels et al., 2005c, Epro et al., 2018a) and slip (Ding and Yang, 2016) perturbations. The leg extensor muscle-tendon units (MTUs) play important roles in locomotion, with the muscles opposing gravity and controlling and generating progression by decelerating and accelerating the centre of mass and the tendons storing and returning elastic energy to the musculoskeletal system (Biewener and Roberts, 2000, Roberts, 2002, Pandy and Andriacchi, 2010). As a consequence, the tendons can also have a large influence on movement effectiveness (Hof et al., 2002, Lichtwark and Wilson, 2007, Pandy and Andriacchi, 2010, Huang et al., 2015). Specifically, the mechanical properties of the Achilles (AT) and patellar (PT) tendons (e.g. tendon stiffness) can greatly influence the contributions of the triceps surae (TS) and quadriceps femoris (QF) to forward propulsion and energy absorption during gait.

Figure 1 displays an overview of recent literature adapted from McCrum et al. (2018b) examining age-related differences in human leg extensor MTU biomechanical properties in young and older healthy adults, including muscle strength and the mechanical (tendon stiffness), morphological (tendon cross sectional area: CSA) and material (Young's modulus of the tendon) properties of the AT and PT.

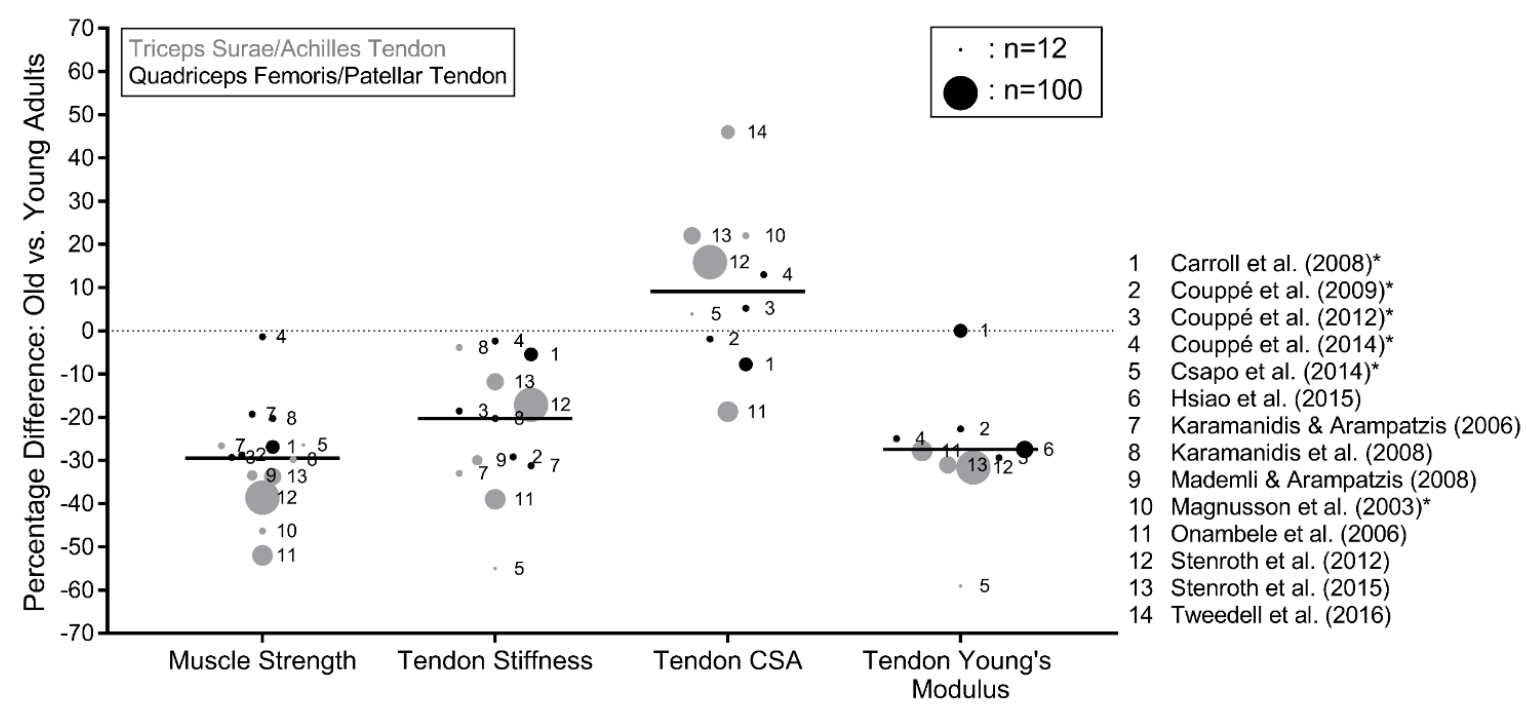

Fig. 1: Percentage differences in triceps surae and quadriceps femoris MTU biomechanical properties between older (mean age of 60y or older) and younger (mean age of 30y or younger) adults reported in the literature. The black lines represent the median values of the means taken from the studies and the circle size is an approximate representation of the relative sample size. ${ }^{*}$ indicates the studies that assessed tendon CSA with magnetic resonance imaging, while the others used ultrasound. Muscle strength was determined during maximum voluntary contractions and reported in the original studies in $\mathrm{kg}, \mathrm{N}, \mathrm{Nm}$ or body weight normalized values. Adapted from McCrum et al. (2018b). 
Based on the available literature, increasing age appears to result in reductions in human TS and QF muscle strength accompanied by reductions in AT and PT stiffness and elastic modulus, whereas the effect on AT and PT CSA is unclear. Therefore, the observed changes in tendon stiffness due to ageing are predominantly due to changes in tendon material properties rather than changes in tendon CSA.

Given the age-related declines in lower limb muscle strength and tendon properties important for force production and locomotion, it is not surprising that interventions aimed at improving these qualities generally show favourable outcomes with regards to falls reduction. Several recent, meta-analyses have estimated that exercise interventions including general muscle strengthening and balance exercises can reduce the number of people who experience a fall in a given year by $17-22 \%$ compared to control groups (Sherrington et al., 2017, Tricco et al., 2017, Hamed et al., 2018, Sherrington et al., 2019). However, due to the multifactorial nature of the interventions included in these metaanalyses, as well as the various potential causes of falls, specific mechanisms behind these results are difficult to determine. One of our recent studies (Epro et al., 2018b) aimed to investigate if improvements in the muscle and tendon properties of the triceps surae, in addition to repeated exposure to cable-trip perturbations, would lead to better trip recovery performance in older adults. Previous work highlighted a link between plantarflexion and trip recovery (Pijnappels et al., 2005b, Pijnappels et al., 2005a, Pijnappels et al., 2005c) and we expected that the combined effects of specific resistance exercise and perturbation exposure would lead to the greatest benefits. Two groups of older women completed a repeated gait perturbation task three times; at baseline, 14 weeks and 1.5 years (8 cable-trip perturbations on each occasion). One of the groups additionally completed resistance exercise, comprised of isometric plantarflexion contractions with a high training load (90\% of one repetition maximum for five sets of four repetitions, two to three times per week) for 14 weeks, with a subgroup continuing for 1.5 years. Despite significant increases in the triceps surae strength (approximately 25\%) after 1.5 years of training, the strength training group appeared to have no additional advantage during the final gait perturbation session compared with the group who only completed the three gait perturbation sessions (Epro et al., 2018b). In alignment with our study, another training study (14 weeks, 2 sessions per week) comparing 1.5-hour sessions of perturbation-based balance training (PBT) with sessions including 45 minutes PBT and 45 minutes muscle strength training in older adults found larger improvements in stability following a gait perturbation task in the 1.5-hours PBT group (Bierbaum et al., 2013). These results seem to agree with recent meta-analyses demonstrating a $39-48 \%$ reduction in the number of people experiencing a fall when higher intensity balance exercise or stability-challenging exercise programmes are followed (Sherrington et al., 2017, Hamed et al., 2018). Exercise programmes focussing specifically on volitional and reactive stepping also show similar reductions at 49\% (Okubo et al., 2017). These reductions far exceed those seen in more general exercise interventions described above (17-22\%), highlighting the importance of training specificity when it comes to falls reduction. 


\subsection{2: Task-specific Balance Assessment and Training}

\subsubsection{1: Specificity}

In 2014, Grabiner et al. published an article titled "Exercise-based fall prevention: can you be a bit more specific?" which succinctly highlights that the assessment and improvement of fall risk through exercise testing and training must be specific to the task. Recommendations that balance training should be task-specific have been given for quite some time (Tang and Woollacott, 1996, Oddsson et al., 2007, Pai and Bhatt, 2007, Maki et al., 2008) and there is now a body of evidence supporting these recommendations. With regards to assessment, it is clear that no single test of balance can capture all balance requirements for daily life (Patla et al., 1990). A recent meta-analysis reported only small correlations between different types of balance performance across the lifespan (Kiss et al., 2018). Ringhof and Stein (2018) have also demonstrated that performance of four different balance tasks seem to measure distinct balance characteristics and only distinguish between swimmers (low postural balance demands) and gymnasts (high postural balance demands) when the test closely resembles movements of one of the sports (e.g. a jump and land test). Regarding falls, standing balance tests in various forms have often been used to assess fall risk, but static and dynamic posturography outcomes could not distinguish previous older fallers from older non-fallers in one study (Baloh et al., 1994) and have repeatedly shown a lack of association with balance recovery performance during dynamic and reactive balance tasks (Owings et al., 2000, Mackey and Robinovitch, 2005, Bhatt et al., 2011, McCrum et al., 2017a).

Like balance assessment, balance training also yields task specific improvements. One systematic review has demonstrated a task-specific effect of balance training in healthy adults and children, whereby trained tasks improve with practice, but untrained tasks do not generally improve (Kummel et al., 2016). In studies on perturbation-based balance training (PBT), specific improvements in dynamic and perturbed balance tasks are observed, as expected, but no or minimal changes are seen in less dynamic or static balance tasks (Freyler et al., 2016, Chien and Hsu, 2018, Krause et al., 2018), although one study has reported beneficial transfer effects from PBT to standing balance (Kurz et al., 2016). Accordingly, it is not surprising that PBT leads to better performance of rapid balance control tasks than traditional proactive balance control training (Freyler et al., 2016) and Tai-Chi training (Aviles et al., 2019). As discussed in Chapter 5.1, PBT for falls prevention concords more to the principles of specificity in training and learning than does traditional balance training, so these results should not be surprising, and I recently attempted to recapitulate this:

"We do not expect athletes to win Olympic medals without practising the skills of their sports, so why should we expect older adults to fall less often without practising the skills required for avoiding falls?" McCrum (2018)

\subsubsection{2: Generalisability}

The concept of specificity in exercise-based falls prevention, while clear and principled, may be, in some sense, a double-edged sword, as it concurrently implies that training will 
be less generalisable. This deserves consideration, as the primary reason for simulating fall-like losses of balance in PBT is to increase the generalisability of the training to daily life. However, as falls can occur due to a multitude of different perturbations (in magnitude, type, direction, limb disturbed, walking environment, etc.), the question is how we can maximise the generalisability of our highly specialised PBT tasks?

Partial generalisability of adaptations in the recovery response to repeated treadmill-delivered trip and slip-like perturbations to untrained trip and slip perturbations during overground walking has been reported (Grabiner et al., 2012, Wang et al., 2019). Additionally, slip-like perturbations delivered via moveable platforms on a walk way lead to adaptations that could benefit balance recovery following slipping on an oily surface (Bhatt and Pai, 2009). Similarly, trip-related falls incidence in daily life was reduced in one study after four sessions of treadmill-based trip-like perturbation training (Rosenblatt et al., 2013). Together, these studies indicate that experiencing repeated balance perturbations can benefit recovery performance following other, untrained perturbations during walking, if the direction of balance loss, the balance recovery mechanisms used and potentially the characteristics of the perturbation are similar. However, the extent of the similarity in these components required for successful generalisation remains to be accurately determined. As discussed in Chapter 5.1, adaptations to perturbations in one direction do not necessarily benefit balance recovery following perturbations in other directions, even when the perturbation type and magnitude, and therefore the balance recovery mechanisms required are similar (see also: Dijkstra et al., 2015; Peterson et al., 2016). However, whether conducting perturbations in multiple directions during a training session might have an interference effect on the adaptation in balance recovery or the retention of those adaptations has not been thoroughly examined. One study in young adults indicated minimal interference between adaptation to repeated slip perturbations and subsequent recovery performance following a trip (Bhatt et al., 2013) and another reported improvement in the recovery from both trips and slips in a mixed perturbation protocol in young adults (Okubo et al., 2018).

The results presented in Part Four of this dissertation suggest that interlimb transfer of adaptations (which also indicates generalizability) following repeated perturbations to one limb might depend on the initial responses to the perturbations and the extent to which the system estimates that adaptations in the response and the transfer of those adaptations is required or necessary. This might suggest that perturbations of a high magnitude, relative to the capacities of a participant, might be more likely to stimulate generalisable adaptations. A number of other studies seem to support this notion. Larger platform slip distances experienced during training (18cm versus $12 \mathrm{~cm}$ ) led to better recovery performance following an unconstrained platform slip (up to $150 \mathrm{~cm}$ ) in one study with young adults (Yang et al., 2014). In studies using a similar protocol on a treadmill, treadmill accelerations at $12 \mathrm{~m} / \mathrm{s}$ (Liu et al., 2016) but not $9 \mathrm{~m} / \mathrm{s}$ (Lee et al., 2016) result in improved generalisability of adaptations to an unconstrained platform slip in young adults compared to accelerations at $6 \mathrm{~m} / \mathrm{s}$. Additionally, as well as increasing perturbation magnitude, increasing the number of perturbations during training may also have a similar effect, as two studies found an increase in generalisability from treadmill- 
delivered perturbations to overground perturbations with an increased volume of treadmill perturbations during the training period (Yang et al., 2013, Yang et al., 2018a).

A final method to better understand how to optimise generalisability of PBT is to use an ecological approach, for example, investigating overground and stair climbing gait on a moving bus (Karekla and Tyler, 2018b, Karekla and Tyler, 2018a) or analysing the reactive stability of train conductors who experience ground surface translations on a daily basis (Baumgart et al., 2016). Such studies may, in the future, provide more specific insight into dynamic balance control and its adaptation in real life situations that represent a risk of falls to older adults.

\subsubsection{3: Other Task-specific Targets for Fall Reduction Interventions}

Reactive balance recovery reactions are of great importance for fall prevention, but PBT could also incorporate, or be combined with, a few other task-specific training categories that all may contribute to safer locomotion in older adults. In the following sections, three specific potential targets are described, and Fig. 2 illustrates how each potentially contributes to the ability to cope with perturbations during walking.

\section{Foot Placement and Gait Adaptability}

Regarding avoiding obstacles during gait, older adults demonstrate more errors during perturbations in visually cued walking than younger adults (Mazaheri et al., 2015, Potocanac et al., 2015), and within older adults, prospective fallers perform worse on multiple visual cue-directed walking tasks such as obstacle avoidance, sudden stops, goaldirected stepping and turning tasks (Geerse et al., 2019). Older, compared to younger adults also have a reduced ability to adjust stepping during gait initiation when foot placement targets are shifted, and consequently have a slower return to stable gait (Sun et al., 2017). However, there may be potential to improve foot placement adjustments with training, as there is evidence that the avoidance of "forbidden landing zones", represented by coloured projections onto the ground, during trip recovery can be improved with practice in young adults (Potocanac et al., 2014). Improving adaptability of foot placement could potentially reduce the risk of falls by avoiding dangerous objects or surfaces altogether, as well as improving the ability to select safe foot placement locations during periods of instability (Fig. 2).

\section{Gait Robustness}

As well as studying the reactive responses following balance loss, it is important to consider how the balance loss occurs and if the robustness of the gait pattern to perturbations can be improved. In this context, rather than just using large perturbations that bring people out of balance, applying smaller perturbations during gait that do not require a complete switch of locomotor behaviour may also lead to positive improvements in gait stability via an increased robustness of the movement patterns. Both coping with small perturbations without a significant change in gait behaviour and with large perturbations that do require some explicit recovery movements have previously been suggested as key requirements for stable gait (Bruijn et al., 2013), and both show 
significant declines with increasing age (Maki and McIlroy, 2006, Süptitz et al., 2013, Terrier and Reynard, 2015, McCrum et al., 2016a). The importance of studying stability during steady-state gait, in addition to reactive stability during larger perturbations, is supported by evidence of the relationship between decreased stability during steady state gait and falls incidence (Hausdorff et al., 2001, van Schooten et al., 2016, Bizovska et al., 2018).

Through the application of small perturbations during steady state walking, the stability of specific locomotion attractors may be modified. Attractors represent coordination tendencies among system components (Davids et al., 2008), can be identified at multiple levels and emerge from the self-organization of the lower and higher-level components through circular causality (Haken, 1987). This means that the behaviour of components at a higher level will be influenced (i.e., constrained) in a bottom-up manner by the behaviour of components at the lower level and vice versa. One study has demonstrated alterations in motor primitives while walking and running on uneven, compared with even surfaces, creating activation patterns that were more robust to the perturbations (Santuz et al., 2018). If the basins of attraction of locomotion attractors could be modified in older adults, this could mean that perturbations of a larger magnitude (or perhaps greater frequency or unpredictability) could be accommodated without significant loss in dynamic stability and therefore changes in the gait pattern. For example, while walking over uneven ground, more frequent or larger undulations in the surface could be negotiated without loss of dynamic stability and without the need for subsequent large reactive balance corrections. One recent study had older participants walk on a treadmill with stable and unstable (water) loads in a backpack (Walsh et al., 2018). As would be expected, step variability was increased, and mediolateral dynamic stability decreased in the unstable load condition and electromyography activity was also increased to cope with the load (Walsh et al., 2018). If practiced over longer time periods, a more robust gait pattern may be the result via alterations such as step width or time, joint moments at the ankle to control centre of mass velocity and muscle activation and motor primitives at a mesoscopic level. Further research is needed to examine the training effects of walking with small continuous unexpected perturbations and whether this translates to a more robust response to large perturbations and subsequently reduced falls risk, but such training represents one interesting avenue for future falls prevention interventions.

\section{Landing}

Given that the duration of falls (i.e. time from balance loss until contact with the ground) usually allows sufficient time to plan and make voluntary movements (van Swigchem et al., 2009), it may be possible to train people to land in a way that reduces injury risk (Robinovitch et al., 2003, Groen et al., 2010a, Moon and Sosnoff, 2017, Robinovitch, 2018). One study on trained judo athletes has demonstrated that martial arts falling techniques may reduce the risk of fall-related fractures (van der Zijden et al., 2012) and based on measurements in young adults, learning these techniques may even be safe for people with osteoporosis, if certain safety measures are taken (Groen et al., 2010b). However, most studies have been conducted using expected or self-initiated falls and there are indications 


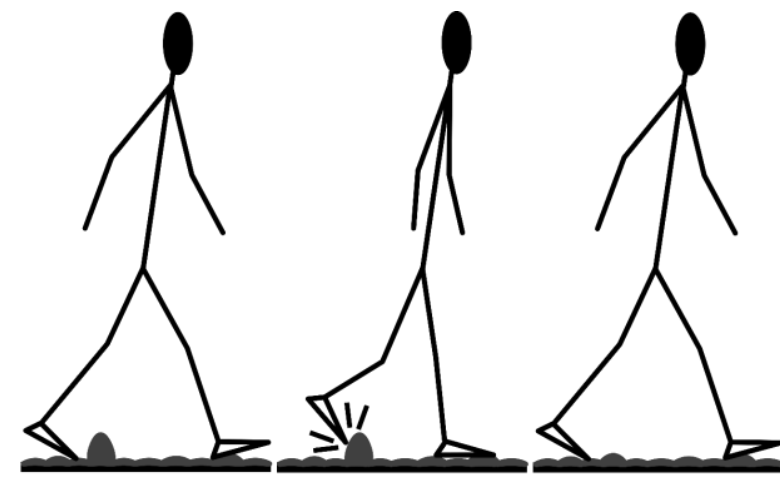

Gait "robustness" to perturbations in the walking

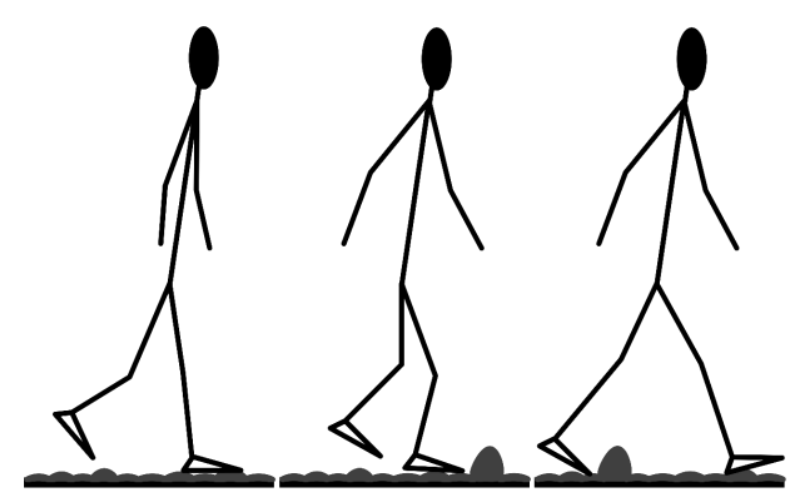

Foot placement adaptability to avoid potential balance threats

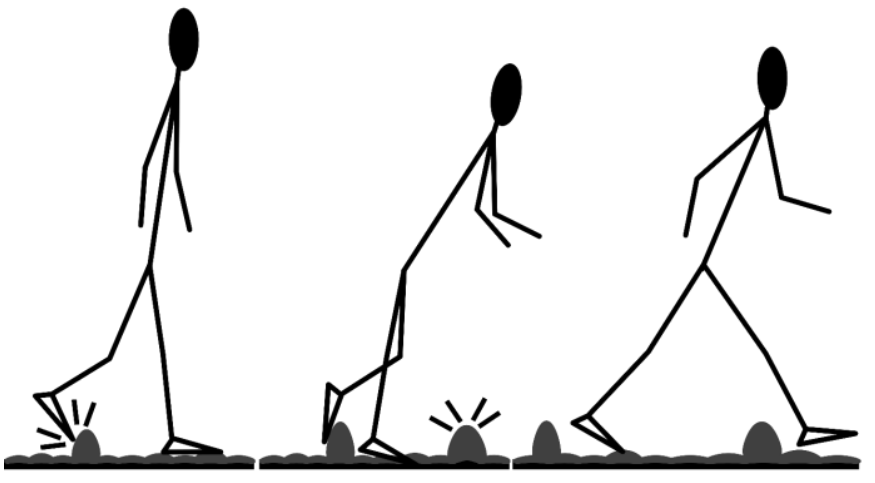

Reactive recovery step following a trip with rapid foot placement adjustment during recovery to avoid a second balance threat

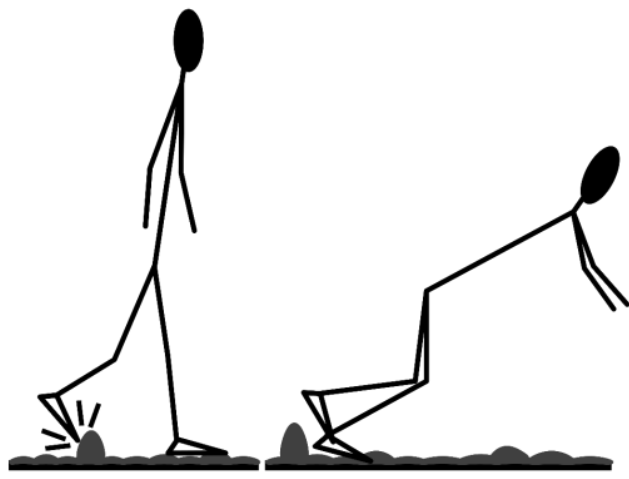

Rapid adjustments to reduce landing impact

Fig. 2: Schematic illustrating the potential contribution of gait robustness, foot placement adaptability before and after balance loss and safe landing skills to dealing with perturbations occurring during walking.

that athletes trained and experienced in falling safely do not tend to perform any better in this regard when exposed to an unexpected balance perturbation with which they have no prior experience (Feldman and Robinovitch, 2007), indicating that such skills are also task specific. Nevertheless, this approach represents another task-specific exercise-based method that may be beneficial in specific populations, possibly more so in populations with a higher risk or frequency of fall-related injury.

\section{3: Challenges in Implementing Fall Risk Assessment and Reduction Interventions}

A major challenge of exercise-based falls prevention programmes is that most general exercise programmes require relatively long term and consistent participation to achieve physiological adaptations that translate to a reduced risk of falls. In the meta-analyses described above, exercise programmes of 5 to 130 weeks were included, typically with 1-3 sessions per week, and a weekly training time of 3 hours or more typically showing larger effects (Sherrington et al., 2017, Hamed et al., 2018, Sherrington et al., 2019). To have any meaningful impact on population level falls statistics, this implies a huge logistic 
undertaking to have the facilities, staff and financial support to provide even a proportion of our populations with such extensive interventions. A related challenge is that many older adults may not consider falls prevention interventions to be worth participating in, even with a hypothetical $0 \%$ fall risk afterwards, due to the perceived costs and inconvenience of participating, as well as the view that they are not in need of such an intervention (Chou et al., 2006, Hill et al., 2014, Franco et al., 2015, Franco et al., 2016, Kiami et al., 2019). Even among older adults who have received medical treatment due to a fall, participation in falls prevention initiatives appears to be minimal (Shankar et al., 2017). Perturbation-based balance training may partly tackle some of these issues, as significant and long-lasting improvements in reactive balance can be achieved with only one or two training sessions (Bhatt et al., 2012, Pai et al., 2014a, Pai et al., 2014b, Epro et al., 2018b). However, other challenges in implementation lie with healthcare practitioners, rather than patients.

Multiple studies have shown that the majority of general practitioners do not specifically assess older patients' risk of falls unless a fall has already occurred (Wenger et al., 2003, Jones et al., 2011, Grant et al., 2015, Kielich et al., 2017, Mackenzie and McIntyre, 2019) and there is a general lack of awareness and use of recognised clinical guidelines and programmes (Jones et al., 2011, Howland et al., 2018). It is, however, reassuring that fall risk screening and fall reduction behaviours among primary care providers can be significantly improved through various interventions (Brown et al., 2005, Wenger et al., 2009, Tricco et al., 2019). Along similar lines, various surveys of physiotherapists concerning balance assessment practices indicate that reactive postural control is the least regularly assessed component of balance (Sibley et al., 2011, Oates et al., 2017) and when it is assessed, this is usually done using non-standardised methods or tests that do not specifically or completely focus on reactive postural adjustments (Sibley et al., 2013). However, similar to studies in general practice, there is evidence that interventions to encourage standardised reactive balance assessment can increase therapists' use and reporting of reactive balance assessment in clinical settings (Sibley et al., 2018). While these challenges of implementation are outwith the scope of this dissertation, these issues should continue to be investigated in order to improve the number of older adults that receive assessment and training of their reactive balance control.

\section{4: Future Directions in Specific Populations}

This dissertation and the discussion so far have largely focussed on the assessment and improvement of gait stability in healthy older adults. However, a few other populations require some further discussion. In this section, the current state of research and future perspectives for research in the assessment and improvement of gait stability in bilateral vestibulopathy and neurological disease more broadly will be briefly outlined.

\subsection{1: Bilateral Vestibulopathy}

As described in Chapter 2.1, one specific population where vestibular function is severely affected is bilateral vestibulopathy (BVP). In BVP, partial or complete loss of peripheral 
vestibular function affects the vestibulo-ocular, vestibulo-collic and vestibulo-spinal reflexes, responsible for maintaining stable gaze, head position and posture, respectively (Goldberg et al., 2012, Kingma and van de Berg, 2016, Strupp et al., 2017). Not surprisingly, these patients experience instability of posture and gait, as well as an increased risk of falls (Guinand et al., 2012a, van de Berg et al., 2015, Horak et al., 2016, Lucieer et al., 2016, Schlick et al., 2016, Schniepp et al., 2017, Sprenger et al., 2017, Grill et al., 2018, McCrum et al., 2018c). Additionally, significantly higher falls incidence has been reported in BVP (51.1\% since onset - age range 30-80 years, $\mathrm{n}=45)$ than in unilateral vestibulopathy ( $30 \%$ since onset - age $24-86$ years, $n=70$; Herdman et al., 2000). These findings, as well as outlining the balance and gait related deficits that people with vestibulopathy experience, highlight the importance of the vestibular system for the control of posture and gait more generally. Critically, the majority of people with BVP do not see any improvement in their symptoms over time (Zingler et al. 2009). It is currently unclear if exercise-based falls prevention strategies alone could be effective in improving gait stability in BVP. However, three other types of technical therapeutic intervention may hold some promise (Wuehr et al., 2017). Sensory substitution devices that provide vibrotactile feedback based on the person's movement appear to improve balance and mobility in BVP (Wuehr et al., 2017, Kingma et al., 2018) and noisy galvanic vestibular stimulation has shown some early signs of beneficial effects on balance and gait in healthy adults and in BVP where there is some residual function (Wuehr et al., 2017, Herssens and McCrum, 2019). Finally, recent advances in the vestibular implant in humans are promising, with partial or complete restoration of various vestibular reflexes and functions having already been demonstrated (for further details, see: Perez Fornos et al., 2014, Guinand et al., 2015, Perez Fornos et al., 2017, van de Berg, 2018). However, the role that balance and gait assessment can play in the assessment of people with BVP and in the evaluation of the effectiveness of such interventions requires more investigation. The results presented in Chapter 2.1 indicate that gait variability at specific walking speeds may provide insight into vestibular function that goes beyond the information provided by several typical clinical tests of vestibular function, but further investigations into balance and gait in BVP are needed to explore the potential utility of such assessments for research and clinical applications.

\subsection{2: Neurological Disease}

As outlined in Chapter 5.1, older adults with neurological disorders are at an increased risk of falling over and above that associated with ageing alone (Homann et al., 2013). It is also worth repeating that there is limited evidence that traditional strength and balance exercise interventions reduce the risk of falls in older adults with Parkinson's disease or stroke (Allen et al., 2010, Verheyden et al., 2013, Canning et al., 2015). As described throughout this dissertation, the increased task-specificity of perturbation-based balance training (PBT) may result in greater reductions in falls risk. However, this assumes that adaptations in reactive balance control can be successfully achieved and retained over time. Our group has recently proposed the hypothesis that people with neurological disorders whose sensory input or motor control is affected may have a decreased responsiveness to repeated gait perturbations and that more perturbation trials will be 
needed to stimulate these adaptations, reflecting a rightward shift in the PBT doseresponse relationship (Karamanidis et al., 2019). In turn, the retention and generalizability of these adaptations may be negatively affected in people with neurological disorders, limiting the overall effectiveness of PBT for falls prevention (Karamanidis et al., 2019). It remains an area for future research to experimentally investigate the perturbation dose-response relationship in these patient populations, as well as the underlying neurological factors involved in gait adaptation, which should provide more insight into the potential for PBT to reduce falls in these groups.

\section{5: Conclusions of the General Discussion}

This dissertation aimed to further our understanding of how old age might affect gait stability and adaptability, with the perspective that this knowledge could improve the effectiveness and specificity of exercise-based falls reduction interventions for both healthy and clinical populations at an increased risk of falls. In addition to the conclusions made in the various chapters in this dissertation, this general discussion has proposed several conclusions, perspectives and directions for future research on, and implementation of, the assessment and improvement of gait stability in older adults. While improving general muscle strength is no doubt important for a number of other reasons, the gait stability of older adults, and therefore falls risk, is not necessarily drastically improved with enhancements in muscle strength. Task specific assessment and training of gait and balance is required to have insight and positive influence on the risk of falls in older adults. Future research must continue explore how to optimise the effectiveness of PBT in terms of generalisability to daily life balance challenges. Other task specific skills such as gait robustness, foot placement and obstacle avoidance and safe landing may be complimentary to PBT and result in an overall greater effect on falls incidence and fall-related injuries. Finally, a number of challenges in improving the fall risk of people with BVP and other neurological disorders exist and the effects of technical therapeutic interventions, as well as task specific exercise-based training of balance and gait in these patients should continue to be investigated. 


\section{Part Seven:}

Summaries and Valorisation 


\section{1: English Summary}

This dissertation aimed to further our understanding of how old age might affect gait stability and adaptability, with the perspective that this knowledge could improve the effectiveness and specificity of exercise-based falls reduction interventions for both healthy and clinical populations at an increased risk of falls.

Part One of this dissertation reviewed the incidence, causes and consequences of falls among older adults. Humans are living longer than ever before and the percentage of older people in our populations will continue to rise for the foreseeable future. As a result, age-related health risks and disease are becoming a larger and larger burden on our older populations and our societies as a whole. Falls and their consequences are one of the most common risks to health, mobility and quality of life among older adults and the number of injuries and hospital admissions because of falls have been increasing in recent years. In particular, mechanical perturbations such as trips und slips during walking present a significant risk for falls in older adults. Therefore, Part One concluded that examination of the stability and adaptation of gait during such mechanical perturbations may help improve the effectiveness of interventions aimed at reducing falls, by identifying specific mechanisms of gait adaptation that can be exploited.

Part Two of this dissertation examined gait variability and stability during walking tasks targeting predominantly predictive control during unperturbed and perturbed gait. Chapter 2.1 assessed the gait of young and older healthy adults, as well as adults with bilateral vestibulopathy, during multiple walking speeds, to elucidate the potential role of the vestibular system in unperturbed gait control. The findings demonstrated that walking speed significantly affects multiple gait parameters and their variability in all the participant groups, and potentially influences the vestibular contribution to locomotor control. Chapter 2.2 used a sustained resistance perturbation to explore the predictive adaptation of gait to control stability in young, middle-aged and older adults, to investigate how such control might change across the adult lifespan. Following a certain number of repeated perturbations, the older adults could achieve similar stability values to younger adults, but the rate of adaptation to a sustained perturbation was found to be slower in the older adults.

Part Three of this dissertation examined methodological aspects of reactive gait stability assessment using perturbations and how we might improve our experimental procedures to better understand how people control and adapt their gait on exposure to unexpected perturbations. Chapter 3.1 reviewed previous studies and their methodologies for examining reactive gait stability during unexpected perturbations in older adults and made suggestions regarding what methodological aspects required further investigation and regarding considerations for future research. In response to the outcomes of the literature review, Chapter 3.2 confirmed that walking speed significantly affects the stability of the body configuration during walking and presented a method to reduce interindividual differences in gait stability prior to experiencing a perturbation, allowing a theoretically more accurate examination of responses to gait perturbations and any subsequent adaptations in gait. 
Part Four of this dissertation subsequently applied the improved methodology to examine how young humans adapt, retain and transfer adaptations in gait stability to controlled gait perturbations (Chapter 4.1) and whether or not the processes of adaptation and transfer are altered in older adults (Chapter 4.2). Furthermore, Chapter 4.2 investigated whether or not having young and older participants walk with equivalent gait stability using the methods presented in Chapter 3.2 would lead to different conclusions than those previously reported in the literature regarding age-related differences in gait stability. The results demonstrated that the relationship between walking speed and stability varies from person to person in both young (Chapter 4.1) and older adults (Chapter 4.2) and that older adults could demonstrate interlimb transfer of adaptations to sudden perturbations (Chapter 4.2) when younger adults did not (Chapter 4.1).

Part Five reviewed the literature to examine the characteristics of perturbationbased balance training studies conducted with older adults that assessed prospective falls incidence and discussed several considerations for applying perturbation-based balance training in clinical settings. The results indicated that perturbation-based balance training is a feasible approach to reduce falls risk in healthy and clinical populations and the most feasible methods appear to be treadmill-based systems and therapist-applied perturbations. While more research into specific perturbation characteristics is necessary, using perturbations of multiple types, directions and magnitudes seems advisable based on the reviewed literature.

Part Six of this dissertation discussed the results of all parts of this dissertation within the broader context of exercise-based falls prevention and a number of conclusions were drawn. While improving general muscle strength is important for older adults for several other health and function-related reasons, the gait stability of older adults, and therefore falls risk, is not necessarily drastically improved with enhancements in muscle strength through resistance training. Task specific assessment and training of gait and balance is required in order to gain insight into, and positively influence, the risk of falls in older adults. Despite the strong evidence for these points, future research must continue to explore how to optimise the effectiveness of perturbation-based balance training in terms of retention and generalisability to daily life balance challenges. Other task specific skills such as gait robustness, gait or foot placement adaptability and safe landing techniques may be complimentary to perturbation-based balance training and result in an overall greater effect on falls incidence and fall-related injuries. Several challenges in improving the fall risk of people with bilateral vestibulopathy and other neurological disorders exist related to their residual function and capacity to respond to physical exercise interventions. To this end, the effects of ever-improving technical therapeutic interventions, as well as task specific exercise-based training on balance and gait in these patients should continue to be investigated. 


\section{2: Nederlandse Samenvatting}

In het kader van valpreventie is kennis omtrent de invloed van leeftijd op de stabiliteit en het aanpassingsvermogen tijdens het lopen essentieel. Middels dit proefschrift is getracht de kennis hieromtrent te vergroten met als doel de effectiviteit en de specificiteit van valpreventieve oefenprogramma's te optimaliseren voor personen met een verhoogd valrisico.

In deel een van dit proefschrift worden de incidentie, alsmede de oorzaken en consequenties van vallen bij oudere patiënten beschreven. Vanwege de vergrijzing verandert de leeftijdssamenstelling van de bevolking en neemt het aandeel van oudere leeftijdsgroepen de komende jaren aanzienlijk toe. Als gevolg hiervan neemt ook het aantal leeftijdsgebonden gezondheidsrisico's en ziekten toe. De negatieve impact van deze ziektebeelden op de kwaliteit van leven, alsmede op de maatschappij is groot. Vallen, en de gevolgen hiervan, hebben grote, negatieve consequenties ten aanzien van algemene gezondheid, mobiliteit en de kwaliteit van leven voor oudere personen. Het aantal verwondingen en ziekenhuisopnames ten gevolge van een val zijn dan ook aanzienlijk toegenomen de afgelopen jaren. Valincidenten bij ouderen worden voornamelijk veroorzaakt door mechanische verstoringen tijdens het lopen (bijvoorbeeld struikelen en uitglijden). Derhalve wordt in deel een geconcludeerd dat onderzoek naar de stabiliteit en vermogen tot aanpassing van het looppatroon tijdens dergelijke verstoringen van groot belang is om de effectiviteit van valpreventieve interventies te verbeteren. Een beter begrip van de specifieke mechanismen achter loopaanpassingen is hiervoor een vereiste.

In deel twee van dit proefschrift wordt de loopvariabiliteit en loopstabiliteit tijdens verschillende looptaken, met en zonder externe verstoringen, onderzocht. In hoofdstuk 2.1 is het looppatroon bij verschillende loopsnelheden van gezonde volwassenen (jong en oud), alsmede van volwassenen met bilaterale vestibulopathie onderzocht, om zo de mogelijke rol van het vestibulaire systeem bij een onverstoord looppatroon te verduidelijken. De resultaten tonen aan dat loopsnelheid een grote invloed heeft op de verschillende loopparameters en hun variabiliteit in alle deelnemende groepen, en dat loopsnelheid mogelijk ook de bijdrage van het vestibulaire systeem aan de motorische controle beïnvloedt. Hoofdstuk 2.2 beschrijft het vermogen om het looppatroon aan te passen als reactie op een constante weerstand tijdens de zwaaifase bij jonge, middelbare en oudere personen. $\mathrm{Na}$ een reeks herhalingen tonen alle drie de leeftijdsgroepen een vergelijkbare loopstabiliteit, echter ouderen passen zich aanzienlijk langzamer aan na een verstoring dan jongeren.

In deel drie van dit proefschrift worden de methodologische aspecten van het onderzoek naar reactieve loopstabiliteit middels het gebruik van verstoringen onderzocht. Dit hoofdstuk bediscussieert op welke manier we onze experimenten kunnen optimaliseren om meer inzicht te krijgen in de controle en de aanpassingen van het looppatroon bij volwassenen. In hoofdstuk 3.1 wordt een overzicht gepresenteerd van eerder uitgevoerde studies en methoden om de reactieve loopstabiliteit van ouderen ten gevolge van onverwachte verstoringen te besturen. Op basis hiervan zijn aanknopingspunten voor verder onderzoek geformuleerd. In navolging van deze literatuurstudie wordt in hoofdstuk 3.2 aangetoond dat loopsnelheid de dynamische 
stabiliteit beïnvloedt. Daarnaast wordt een methode gepresenteerd die de interindividuele verschillen in loopstabiliteit vermindert en daardoor het onderzoek naar verstoringen in het gangbeeld meer accuraat maakt.

In deel vier van dit proefschrift wordt gebruik gemaakt van de eerder beschreven, geoptimaliseerde methodologie om te onderzoeken hoe jongeren hun loopstabiliteit controleren en aanpassen (hoofdstuk 4.1). Ook is gekeken of dit verschilt van ouderen (hoofdstuk 4.2). Daarnaast wordt in hoofdstuk 4.2 onderzocht of de reeds in literatuur gerapporteerde resultaten met betrekking tot leeftijdgerelateerde verschillen in loopstabiliteit kunnen worden bevestigd met behulp van de methode die wordt gepresenteerd in hoofdstuk 3.2. De resultaten tonen een intra-individuele variabiliteit aan van de relatie tussen loopsnelheid en loopstabiliteit voor zowel jongere (hoofdstuk 4.1), evenals oudere volwassenen (hoofdstuk 4.2); Bovendien wordt aangetoond dat alleen bij oudere volwassenen een tranfer plaatsvindt van de aanpassingen van het getrainde been naar het ongetrainde been.

De literatuurstudie in deel vijf geeft meer inzicht in de kenmerken van op verstoring gebaseerde balanstraining bij ouderen met een verhoogd valrisico; Daarnaast wordt in deel vijf ook de toepassing hiervan voor de kliniek bediscussieerd. Resultaten tonen aan dat balanstraining met behulp van verstoringen een geschikte manier is om het valrisico voor gezonde en klinische populaties te verlagen (waarbij training op een loopband danwel met een therapeut het meest geschikt lijkt). Hoewel verder onderzoek nodig is naar specifieke verstoringskenmerken, toont de huidige literatuur aan dat een diversiteit van mechanische verstoringen (in multipele richtingen, van verschillende grootten en verschillende soorten) het best gebruikt kan worden.

In deel zes van dit proefschrift worden alle resultaten van dit proefschrift binnen de bredere context van valpreventieve training beschreven en worden een aantal conclusies getrokken. Hoewel het verbeteren van de spiercapaciteit een positieve invloed heeft op de lichaamsfuncties en de algehele gezondheid, verbetert dit niet noodzakelijkerwijs de loopstabiliteit van ouderen en verlaagt dit dus ook niet het valrisico. Taakgerichte analyse en taakgerichte loop- en balanstraining is nodig om meer inzicht te krijgen in het valrisico bij ouderen alsmede dit op een positieve manier te beïnvloeden. Ondanks dit overtuigende bewijs is verder onderzoek nodig om de effectiviteit van verstorings-gebaseerde evenwichtstraining te optimaliseren bij het handhaven en overbrengen van leereffecten naar de dagelijkse uitdagingen in de loopbalans. Andere parameters, zoals neuro-motorische bewegingscontrole, een gecontroleerde voetplaatsing en veilige val- en landingstechnieken moeten hand in hand gaan met op verstoring gebaseerde balanstraining om het positief effect op valrisico of valgerelateerd letsel te vergroten. Vooral het onderzoek ten aanzien van het verlagen van het valrisico van personen met bilaterale vestibulopathie en andere neurologische ziekten kent verschillende uitdagingen door bijvoorbeeld een verminderd aanpassingsvermogen en een verminderde fysieke belastbaarheid. Daartoe moeten de effecten van steeds betere technische en therapeutische opties en taakspecifieke trainingsprogramma's voor lopen en balans verder worden onderzocht in deze patiëntengroep. 


\section{3: Deutsche Zusammungfassung}

Die vorliegende Dissertation zielt darauf ab unser Verständnis zum Einfluss von Alter auf die Gangstabilität und Ganganpassungsfähigkeit zu erweitern. Damit sollen die neu gewonnenen Erkenntnisse zu einer Verbesserung von Effektivität und Spezifität spezieller Trainingsmaßnahmen zur Prävention von Stürzen für sowohl gesunde als auch klinische sturzgefährdete Bevölkerungsschichten führen.

Teil Eins dieser Dissertation überprüft die Epidemiologie, Ätiologie und Konsequenzen von Stürzen bei älteren Personen. Die Lebenserwartung steigt zunehmend und der Anteil älterer Menschen innerhalb der Bevölkerung wird in Zukunft weiterhin ansteigen. Infolgedessen werden altersbedingte Gesundheitsrisiken und Krankheiten ein immer größeres Problem für die Älteren und die Gesellschaft als Ganzes. Stürze und deren Folgen zählen zu den häufigsten Risiken für Gesundheit, Mobilität und Lebensqualität Älterer und die Anzahl sturzbedingter Verletzungen und Krankenhausaufenthalte sind in den letzten Jahren weiter gestiegen. Vor allem mechanische Störungen (Perturbationen) während des Gehens (z.B. Stolpern und Ausrutschen) stellen eine erhebliche Sturzgefahr für ältere Personen dar. Teil Eins gelangt zu der Erkenntnis, dass eine Untersuchung der Gangstabilität und Ganganpassung während solcher mechanischer Perturbationen hilft, die Effektivität von Interventionen zur Sturzprävention zu verbessern indem spezifische Mechanismen der Ganganpassung identifiziert und in Folge genutzt werden.

Teil Zwei dieser Dissertation untersucht die Gangvariabilität und Gangstabilität während unterschiedlicher Gangaufgaben, die vor allem auf die prädiktive Bewegungskontrolle mit und ohne externer Perturbationen abzielt. Kapitel 2.1 untersucht dabei den Gang von jungen und älteren Erwachsenen sowie Erwachsenen mit bilateraler Vestibulopathie während unterschiedlicher Ganggeschwindigkeiten, um die potentielle Rolle des vestibulären Systems für die Gangkontrolle zu analysieren. Die Ergebnisse zeigen, dass die Ganggeschwindigkeit verschiedene Gangparameter und deren Variabilität in allen teilnehmenden Gruppen erheblich beeinflusst und möglicherweise Auswirkungen auf den Einfluss des vestibulären Systems auf die Bewegungskontrolle nimmt. Kapitel 2.2 untersucht die prädiktive Anpassung des Gehens in Reaktion auf einen konstanten Widerstand für junge, mittelalte und ältere Personen und somit deren Veränderung über die Lebensspanne des Erwachsenenalters. Nach einer Reihe von Wiederholungen wiesen alle drei Altersgruppen ähnliche Werte für die Gangstabilität auf. Jedoch erfolgte die Anpassung an die konstante Perturbation langsamer für die älteren im Vergleich zu den jüngeren Erwachsenen.

Teil Drei dieser Dissertation untersucht methodologische Aspekte der Analyse der reaktiven Gangstabilität mit Hilfe von Perturbationen und diskutiert wie solche experimentellen Designs zu verbessern sind, um unser Verständnis von Stabilitätskontrolle in Folge unerwarteter Störungen zu erweitern. Kapitel 3.1 gibt eine Übersicht über vorherige Studien und Methoden zur Untersuchung der reaktiven Gangstabilität von älteren Personen in Folge unerwarteter Perturbationen. Hierbei werden konkrete Vorschläge abgeleitet, welche methodologischen Aspekte einer weiteren Untersuchung und Berücksichtigung bedürfen. Im Hinblick auf die Literaturanalyse 
konnte Kapitel 3.2 bestätigen, dass die Ganggeschwindigkeit die dynamische Stabilität beeinflusst. Weiterhin wurde eine Methode vorgestellt, mit Hilfe derer interindividuelle Unterschiede in der Gangstabilität reduziert und somit eine genauere Untersuchung der reaktiven Stabilitätskontrolle in Folge einer unerwarteten Störung erlaubt.

Teil Vier dieser Dissertation wendet die zuvor genannte verbesserte Methode an, um die Fähigkeit jüngerer Personen zu untersuchen, die Gangstabilität in Folge kontrollierter Perturbationen anzupassen und diese Anpassungen beizubehalten und zu transferieren (Kapitel 4.1). Außerdem wird der Effekt des Alters auf die Anpassung und den Transfer von Lerneffekten analysiert (Kapitel 4.2). Weiterhin untersucht Kapitel 4.2 ob die in früherer Literatur berichteten Ergebnisse hinsichtlich der altersbedingten Unterschiede in der Gangstabilität unter Verwendung der in Kapitel 3.2 vorgestellten Methode bestätigt werden können. Die Ergebnisse zeigen eine intraindividuelle Variabilität des Zusammenhangs von Ganggeschwindigkeit und Gangstabilität sowohl für junge (Kapitel 4.1), als auch ältere Erwachsene (Kapitel 4.2) und dass ausschließlich ältere Erwachsene in der Lage waren einen Transfer von Anpassungseffekten zwischen den Beinen auszubilden.

Teil Fünf gibt eine Übersicht zu den Charakteristiken von perturbationsbasiertem Gleichgewichtstraining bei älteren Personen, das eine Aussage über das zukünftige Sturzrisiko zulassen soll. Dabei wurden verschiedene Überlegungen für die Anwendung eines solchen Trainings in der Klinik diskutiert. Die Ergebnisse deuten darauf hin, dass Gleichgewichtstraining unter zu Hilfenahme von Perturbationen eine geeignete Herangehensweise darstellt, um das Risiko von Stürzen für gesunde und klinische Bevölkerungsgruppen zu reduzieren. Als praktikabelste Methode konnten Laufbandsysteme und von Therapeuten durchgeführte Perturbationen identifiziert werden. Obwohl es weiterer Forschung zu konkreten Perturbationsmerkmalen bedarf, scheinen, basierend auf der international einschlägigen Literatur, unterschiedliche Arten, Richtungen und Umfänge von mechanischen Perturbationen empfehlenswert zu sein.

Teil Sechs diskutiert die Ergebnisse aller Teile dieser Dissertation im Kontext trainingsbasierter Maßnahmen zur Prävention von Stürzen und unter Ableitung finaler Schlussfolgerungen. Während eine Verbesserung muskulärer Kapazitäten einen positiven Einfluss auf die Körperfunktion und allgemeine Gesundheit nimmt, führt dies nicht zwangsläufig zu einer Verbesserung der Gangstabilität älterer Menschen und somit auch nicht zu einer Reduzierung des Sturzrisikos. Dagegen scheint eine aufgabenspezifische Analyse und Verbesserung von Gang und Gleichgewicht durch Training erforderlich, um noch mehr Erkenntnisse zu bekommen und die Sturzgefahr älterer Menschen positiv zu beeinflussen. Trotz dieser überzeugenden Nachweise sind weitere Untersuchungen nötig, um die Wirksamkeit perturbationsbasierten Gleichgewichtstrainings in Bezug auf die Beibehaltung und den Transfer von Lerneffekten auf tägliche Gleichgewichtsherausforderungen im Alltag zu optimieren. Andere Parameter, wie z.B. die neuromotorische Kontrolle von Bewegung, ein kontrollierter Fußaufsatz, sowie sichere Landungstechniken sollten Hand in Hand mit perturbationsbasiertem Gleichgewichtstraining gehen und einen etwaigen positiven Effekt auf das Sturzrisiko bzw. sturzbedingte Verletzungen sogar noch verstärken. Vor allem im Hinblick auf eine Verminderung des Sturzrisikos von Personen mit bilateraler Vestibulopathie und anderen 
neurologischen Erkrankungen existieren etliche Herausforderungen hervorgerufen durch eine z.T. verminderte Anpassungsfähigkeit and physische Belastungen. Dazu sollten in diesem Patientenkollektiv die Auswirkungen von zunehmend besseren technischen und therapeutischen Möglichkeiten als auch von aufgabenspezifischen Trainingsmaßnahmen für Gang und Gleichgewicht weiterhin untersucht werden. 
7.3: Deutsche Zusammungfassung 


\section{4: Valorisation}

The research described in this dissertation had the general aim to further our understanding of how gait stability and adaptability are affected in older adults, in order to generate new knowledge that could improve the effectiveness and specificity of exercisebased falls reduction interventions for both healthy and clinical populations at an increased risk of falls. Valorisation refers to generating societal or economic value from knowledge and, in this section, the valorisation of the knowledge gained in this dissertation will be outlined in the contexts of societal and economic relevance, target interest groups, translation of knowledge and innovation, and implementation.

\section{Societal and Economic Relevance}

The societal relevance of better understanding and improving older adults' gait stability has been discussed at length in this dissertation because falls represent a major societal burden and their prevalence and associated consequences will continue to increase in our ageing societies. To the individual, falls represent a threat to physical function and independence, and by extension to social engagement and overall quality of life. The benefits of physical activity and social participation for overall health are well established, but these can become even more important as we age, in order to minimise physical and mental decline. Falls and fall-related injuries can severely disrupt this, with recovery (or lack thereof) and bedrest following injury accelerating physical decline and potentially resulting in social isolation, which can be further exacerbated by an increased concern about falling again. To our societies and economies as a whole, reducing falls and fallrelated injuries would help relieve some logistical and financial pressure on our healthcare systems.

However, the above address falls and fall-related injuries, and not specifically our understanding of gait stability and adaptability, which was the focus of this dissertation. The direct relevance of this understanding lies in the potential for optimising falls reduction interventions. There are a number of challenges with implementing exercisebased falls reduction interventions in society. The first is that they are costly, due to the time required (in order to achieve a minimum effective dose) and the other logistical requirements such as locations, staff and equipment. As discussed in Part Six of this dissertation, improving the specificity of these interventions by better understanding how humans can improve their gait stability will likely reduce the intervention time needed to have a meaningful effect on falls risk and may therefore lead to lower overall costs for implementing these interventions. This may also positively influence the second challenge - ensuring sufficient adherence to the interventions to reach the minimum effective dose. It is well known that many adults do not participate in enough regular physical activity to meet the various national and international guidelines, and therefore exercise-based fall reduction interventions may be more likely to have better adherence when they are of shorter duration or frequency. As mentioned throughout this dissertation, training involving only one to four sessions of large, sudden balance disturbance in a secure environment have demonstrated long term improvements (up to 1.5 years) in reactive 
balance control during walking. Improving our understanding of how these effects occur and how to best exploit them by manipulating our training protocols is therefore highly relevant for society-wide implementation.

\section{Relevance to Groups outwith the Scientific Community}

As described in Part Six of this dissertation, fall risk assessment and intervention among medical and health care practitioners may be lacking in a number of areas. Most general practitioners report not assessing their older patients' risk of falls unless a fall has already occurred, and physiotherapists' assessment of reactive postural control appears to be lacking. However, as these professions typically represent a first point of contact regarding the health and mobility of older adults, they should be target groups for knowledge transfer. If improvements can be made in the assessment and early intervention of reactive balance control at these levels by utilising the knowledge generated by this dissertation and other similar research, declines in balance control in older adults may be delayed at the community level.

In addition to medical and health care practitioners, older adults themselves are a target group for knowledge translation. By increasing awareness among older adults about what can be done to reduce falls risk and improve gait stability, this can also stimulate those individuals to seek out opportunities with their healthcare providers or take steps to reduce their own risk through various exercise or non-exercise-based interventions. Finally, the current results and research on exercise-based falls reduction more broadly should be of interest to organisations involved in healthcare and public health policy. Following building scientific evidence, a number of national physical activity guidelines have recently included resistance exercise as an important physical activity component, especially for older adults. Similar emphasis on increasing the amount of challenging and, when feasible, reactive balance training among older adults could have a positive effect on awareness, policy and practice with regards to falls prevention.

\section{Knowledge Translation and Innovation}

In recent years, the number of companies that produce technologies designed for testing and training mobility has increased and, of particular relevance for the work in this dissertation, an increasing number of commercially available treadmill-based systems that can apply controlled perturbations to balance during walking are becoming available. Many of these devices come with built in programmes for testing and training balance control and the results of this dissertation and related work may help to improve the accuracy, validity and effectiveness of these programmes. However, as such devices are only feasible options for larger or wealthier organisations, another important commercial area for knowledge translation are companies that produce relatively inexpensive tools and products that are feasible for the typical physiotherapy practice or rehabilitation centre. In this regard, translation of the underlying principles of the work is critical, especially regarding the retention and transfer of adaptations in gait and balance. Finally, organisations that develop interventions for specific clinical populations (for example, the 
vestibular implant for people with bilateral vestibulopathy) can be guided in their development of such interventions by an improved understanding of the various components of balance control during walking, by more specific gait and balance testing protocols for their target groups and by investigating which components can be successfully improved through intervention.

\section{Implementation}

The first step to translating scientific knowledge is through the publication and dissemination of the work to the scientific community and the public. The majority of the work in this dissertation has been published in peer reviewed journals and in all cases, an open access version of each article has been made available (either the preprint, accepted or published versions). Parts One, Six and Seven of this dissertation are licensed under the CC BY 4.0 License (which permits unrestricted use, distribution, and reproduction in any medium, provided the original work is properly cited) in the hope that the information presented can be more easily and freely shared and applied. The work has been presented and discussed at national and international scientific conferences as well as conferences with a more clinical perspective intended for health care professionals.

Additionally, the author has participated in industry-initiated workshops aiming to share knowledge about the use of technology in the context of assessing and training gait and balance control and has also given presentations for companies themselves. In the near future, public summaries of the work in this dissertation and its relevance to various products for assessing and training balance will become available on the respective company websites. Such initiatives are intended to bring the end users of the products, as well as the developers, closer to the results of the relevant research. The author will continue to work closely with these companies as the research in this area continues.

Finally, outreach activities for the general public will take more precedence in the future. Openly available summaries of current research activities and public presentations for older adults, patient groups and healthcare practitioners will be planned in order to stimulate a direct link with the researchers that initially will help increase awareness of recent developments but will also stimulate feedback channels to help direct and inform future research that accounts for challenges faced in practice.

\section{Personal Perspective}

Almost every published scientific article concerning balance or gait stability begins with a paragraph on the problem of falls. I wonder how often we as researchers actually translate the knowledge we have gained from research to practice, or at least bring it to a point that others can translate and apply the work to address this problem, and how often it is simply an easy justification for our work? Developing sophisticated protocols, parameters and devices to train and assess gait stability is no doubt of fundamental importance but they will have no impact on the wider societal problem of falls unless they can be applied on a broad, public scale, either directly or indirectly. The current incentive system in science may cloud this bigger picture, with publications often being viewed as end products that 
gather citations and further funding, rather than vehicles of knowledge translation for application in broader contexts. I challenge myself and my fellow researchers in this field to frequently reflect on this and to ask ourselves if we are translating the knowledge we have gained, or if we are just padding our CVs? Speaking for myself, I would be utterly ashamed looking back in 30- or 40-years' time if I could not clearly see how my research had helped us to understand and address the problem of falls.

To this end, I believe that open science practices and active community engagement can go a long way. As many groups for whom this research is relevant do not have academic institutional access to paywalled literature, open access publication of research is vital for successful knowledge translation, as is the dissemination of knowledge through other accessible means such as social and traditional media and open workshops and presentations. Open data, software, code and methodology while perhaps of less direct relevance to knowledge translation for the public, help the scientific community evaluate, improve upon and accelerate what is being researched, which will lead to faster and more robust scientific progress that can be translated. Finally, frequent and direct contact with the public, with health care practitioners and with societies and companies to disseminate new knowledge and discuss their needs and challenges will help improve the translation of past, and translatability of future, research.

\section{Concluding Remarks}

Research on falls prevention is, almost by definition, tied to valorisation. Similarly, research on balance and gait that aims to inform, and lead to improvements in, exercisebased falls risk assessment and reduction is closely linked to valorisation. However, it is vital that we as researchers realise that this does not occur automatically. We should actively pursue channels for translating knowledge gained through research to the public, to healthcare practitioners, to companies and to organisations. Only with this translation of knowledge can we justify using the broader societal issues caused by falls as a rationale for our research. 
References 
ABOLHASSANI, F., MOAYYERI, A., NAGHAVI, M., SOLTANI, A., LARIJANI, B. \& SHALMANI, H. T. 2006. Incidence and characteristics of falls leading to hip fracture in Iranian population. Bone, 39, 408-13. doi: 10.1016/j.bone.2006.01.144

AGRAWAL, Y., VAN DE BERG, R., WUYTS, F., WALTHER, L., MAGNUSSON, M., OH, E., SHARPE, M. \& STRUPP, M. 2018. Presbyvestibulopathy: Diagnostic Criteria. J Vestib Res. Available at: http://www.jvr-web.org/images/Presbyvestibulopathy-Diagnostic-Criteria090618ab-MS01.pdf

AHARONOFF, G. B., DENNIS, M. G., ELSHINAWY, A., ZUCKERMAN, J. D. \& KOVAL, K. J. 1998. Circumstances of falls causing hip fractures in the elderly. Clin Orthop Relat Res, 104.

ALEX, D., KHOR, H. M., CHIN, A. V., HAIRI, N. N., OTHMAN, S., KHOO, S. P. K., BAHYAH KAMARUZZAMAN, S. \& TAN, M. P. 2018. Cross-sectional analysis of ethnic differences in fall prevalence in urban dwellers aged 55 years and over in the Malaysian Elders Longitudinal Research study. BMJ Open, 8, e019579. doi: 10.1136/bmjopen-2017-019579

ALEXANDER, R. M. 1989. Optimization and gaits in the locomotion of vertebrates. Physiol Rev, 69, 1199-227. doi: 10.1152/physrev.1989.69.4.1199

ALEXANDER, R. M. 1991. Energy-saving mechanisms in walking and running. J Exp Biol, 160, 55-69.

ALLAIN, T. J., MWAMBELO, M., MDOLO, T. \& MFUNE, P. 2014. Falls and other geriatric syndromes in Blantyre, Malawi: a community survey of older adults. Malawi Med J, 26, 105-8.

ALLEN, N. E., CANNING, C. G., SHERRINGTON, C., LORD, S. R., LATT, M. D., CLOSE, J. C., O'ROURKE, S. D., MURRAY, S. M. \& FUNG, V. S. 2010. The effects of an exercise program on fall risk factors in people with Parkinson's disease: a randomized controlled trial. Mov Disord, 25, 1217-25. doi: 10.1002/mds.23082

ALLUM, J. H., TANG, K. S., CARPENTER, M. G., OUDE NIJHUIS, L. B. \& BLOEM, B. R. 2011. Review of first trial responses in balance control: influence of vestibular loss and Parkinson's disease. Hum Mov Sci, 30, 279-95. doi: 10.1016/j.humov.2010.11.009

ALMAWLAWI, E., AL ANSARI, A. \& AHMED, A. 2011. Prevalence and Risk Factors for Falls Among the Elderly in Primary Healthcare Centers (PHC) in Qatar. Qatar Medical Journal, 20, 12-17.

ALMEGBEL, F. Y., ALOTAIBI, I. M., ALHUSAIN, F. A., MASUADI, E. M., AL SUlAMI, S. L., ALOUSHAN, A. F. \& ALMUQBIL, B. I. 2018. Period prevalence, risk factors and consequent injuries of falling among the Saudi elderly living in Riyadh, Saudi Arabia: a cross-sectional study. BMJ Open, 8, e019063. doi: 10.1136/bmjopen-2017-019063

ANSON, E. \& JEKA, J. 2015. Perspectives on Aging Vestibular Function. Front Neurol, 6, 269. doi: 10.3389/fneur.2015.00269

AOYAGI, K., ROSS, P. D., DAVIS, J. W., WASNICH, R. D., HAYASHI, T. \& TAKEMOTO, T. 1998. Falls among community-dwelling elderly in Japan. J Bone Miner Res, 13, 1468-74. doi: 10.1359/jbmr.1998.13.9.1468

APRIGLIANO, F., MARTELLI, D., MICERA, S. \& MONACO, V. 2016. Intersegmental coordination elicited by unexpected multidirectional slipping-like perturbations resembles that adopted during steady locomotion. J Neurophysiol, 115, 728-40. doi: 10.1152/jn.00327.2015 
APRIGLIANO, F., MARTElLI, D., TROPEA, P., PASQUINI, G., MICERA, S. \& MONACO, V. 2017. Aging does not affect the intralimb coordination elicited by slip-like perturbation of different intensities. J Neurophysiol, 118, 1739-1748. doi: 10.1152/jn.00844.2016

ARAGAO, F. A., KARAMANIDIS, K., VAZ, M. A. \& ARAMPATZIS, A. 2011. Mini-trampoline exercise related to mechanisms of dynamic stability improves the ability to regain balance in elderly. J Electromyogr Kinesiol, 21, 512-8. doi: 10.1016/j.jelekin.2011.01.003

ARAMPATZIS, A., KARAMANIDIS, K. \& MADEMLI, L. 2008. Deficits in the way to achieve balance related to mechanisms of dynamic stability control in the elderly. J Biomech, 41, 1754-61. doi: 10.1016/j.jbiomech.2008.02.022

ARAMPATZIS, A., PEPER, A. \& BIERBAUM, S. 2011. Exercise of mechanisms for dynamic stability control increases stability performance in the elderly. $J$ Biomech, 44, 52-8. doi: 10.1016/j.jbiomech.2010.08.023

ARENA, S. L., DAVIS, J. L., GRANT, J. W. \& MADIGAN, M. L. 2016. Tripping Elicits Earlier and Larger Deviations in Linear Head Acceleration Compared to Slipping. PLoS One, 11, e0165670. doi: 10.1371/journal.pone.0165670

ARSHAD, Q. \& SEEMUNGAL, B. M. 2016. Age-Related Vestibular Loss: Current Understanding and Future Research Directions. Front Neurol, 7, 231. doi: 10.3389/fneur.2016.00231

ASHTON-MILLER, J. A. \& ALEXANDER, N. B. 2009. Biomechanics of Mobility. In: HALTER, J. B., OUSLANDER, J. G., TINETTI , M. E., STUDENSKI, S., HIGH, K. P. \& ASTHANA, S. A. (eds.) Hazzard's Geriatric Medicine and Gerontology. 6th ed. USA: McGraw-Hill.

AVILES, J., ALlin, L. J., ALEXANDER, N. B., VAN MULlEKOM, J., NUSSBAUM, M. A. \& MADIGAN, M. L. 2019. Comparison of treadmill trip-like training versus Tai Chi to improve reactive balance among independent older adult residents of senior housing: a pilot controlled trial. J Gerontol A Biol Sci Med Sci. doi: 10.1093/gerona/glz018

AZAR, G. J. \& LAWTON, A. H. 1964. Gait and Stepping as Factors in the Frequent Falls of Elderly Women. Gerontologist, 4, 83-4. doi: 10.1093/geront/4.2_part_1.83

BACHMAN, J. C. 2013. Specificity vs. Generality in Learning and Performing Two Large Muscle Motor Tasks. Res Q Am Assoc Health Phys Educ Recreation, 32, 3-11. doi: 10.1080/10671188.1961.10762064

BAILEY, I. L. \& LOVIE, J. E. 1976. New design principles for visual acuity letter charts. $A m J$ Optom Physiol Opt, 53, 740-5.

BALOH, R. W., FIFE, T. D., ZWERLING, L., SOCOTCH, T., JACOBSON, K., BELL, T. \& BEYKIRCH, K. 1994. Comparison of static and dynamic posturography in young and older normal people. J Am Geriatr Soc, 42, 405-12.

BARRETT, R. S., CRONIN, N. J., LICHTWARK, G. A., MILLS, P. M. \& CARTY, C. P. 2012. Adaptive recovery responses to repeated forward loss of balance in older adults. J Biomech, 45, 183-7. doi: 10.1016/j.jbiomech.2011.10.005

BAUBY, C. E. \& KUO, A. D. 2000. Active control of lateral balance in human walking. J Biomech, 33, 1433-40.

BAUMGART, C., HOPPE, M. W. \& FREIWALD, J. 2016. Long-Term Adaptations to Unexpected Surface Perturbations: Postural Control During Stance and Gait in Train Conductors. $J$ Mot Behav, 48, 341-7. doi: 10.1080/00222895.2015.1095154

BAUMGARTNER, R. N., KOEHLER, K. M., GALLAGHER, D., ROMERO, L., HEYMSFIELD, S. B., ROSS, R. R., GARRY, P. J. \& LINDEMAN, R. D. 1998. Epidemiology of sarcopenia 
among the elderly in New Mexico. Am $J$ Epidemiol, 147, 755-63. doi: 10.1093/oxfordjournals.aje.a009520

BEKIBELE, C. O. \& GUREJE, O. 2010. Fall incidence in a population of elderly persons in Nigeria. Gerontology, 56, 278-83. doi: 10.1159/000236327

BERG, W. P., ALESSIO, H. M., MILLS, E. M. \& TONG, C. 1997. Circumstances and consequences of falls in independent community-dwelling older adults. Age Ageing, 26, 261-8. doi: 10.1093/ageing/26.4.261

BERGEN, G., STEVENS, M. R. \& BURNS, E. R. 2016. Falls and Fall Injuries Among Adults Aged >l=65 Years - United States, 2014. MMWR Morb Mortal Wkly Rep, 65, 993-998. doi: 10.15585/mmwr.mm6537a2

BERGER, W., DIETZ, V. \& QUINTERN, J. 1984. Corrective reactions to stumbling in man: neuronal co-ordination of bilateral leg muscle activity during gait. J Physiol, 357, 109-25. doi: 10.1113/jphysiol.1984.sp015492

BHANGU, J., KING-KALlimanis, B. L., DONOGHUE, O. A., CARROLL, L. \& KENNY, R. A. 2017. Falls, non-accidental falls and syncope in community-dwelling adults aged 50 years and older: Implications for cardiovascular assessment. PLoS One, 12, e0180997. doi: 10.1371/journal.pone.0180997

BHATT, T., ESPY, D., YANG, F. \& PAI, Y. C. 2011. Dynamic gait stability, clinical correlates, and prognosis of falls among community-dwelling older adults. Arch Phys Med Rehabil, 92, 799805. doi: 10.1016/j.apmr.2010.12.032

BHATT, T. \& PAI, Y. C. 2008. Immediate and latent interlimb transfer of gait stability adaptation following repeated exposure to slips. J Mot Behav, 40, 380-90. doi: 10.3200/JMBR.40.5.380390

BHATT, T. \& PAI, Y. C. 2009. Generalization of gait adaptation for fall prevention: from moveable platform to slippery floor. $J$ Neurophysiol, 101, 948-57. doi: 10.1152/jn.91004.2008

BHATT, T., WANG, T. Y., YANG, F. \& PAI, Y. C. 2013. Adaptation and generalization to opposing perturbations in walking. Neuroscience, 246, 435-50. doi: 10.1016/j.neuroscience.2013.04.013

BHATT, T., WENING, J. D. \& PAI, Y. C. 2005. Influence of gait speed on stability: recovery from anterior slips and compensatory stepping. Gait Posture, 21, 146-56. doi: 10.1016/j.gaitpost.2004.01.008

BHATT, T., YANG, F. \& PAI, Y. C. 2012. Learning to resist gait-slip falls: long-term retention in community-dwelling older adults. Arch Phys Med Rehabil, 93, 557-64. doi: 10.1016/j.apmr.2011.10.027

BIERBAUM, S., PEPER, A. \& ARAMPATZIS, A. 2013. Exercise of mechanisms of dynamic stability improves the stability state after an unexpected gait perturbation in elderly. Age, 35, 190515. doi: 10.1007/s11357-012-9481-z

BIERBAUM, S., PEPER, A., KARAMANIDIS, K. \& ARAMPATZIS, A. 2010. Adaptational responses in dynamic stability during disturbed walking in the elderly. $J$ Biomech, 43, 2362-8. doi: 10.1016/j.jbiomech.2010.04.025

BIERBAUM, S., PEPER, A., KARAMANIDIS, K. \& ARAMPATZIS, A. 2011. Adaptive feedback potential in dynamic stability during disturbed walking in the elderly. J Biomech, 44, 19216. doi: 10.1016/j.jbiomech.2011.04.027 
BIERYLA, K. A. \& MADIGAN, M. L. 2011. Proof of concept for perturbation-based balance training in older adults at a high risk for falls. Arch Phys Med Rehabil, 92, 841-3. doi: 10.1016/j.apmr.2010.12.004

BIERYLA, K. A., MADIGAN, M. L. \& NUSSBAUM, M. A. 2007. Practicing recovery from a simulated trip improves recovery kinematics after an actual trip. Gait Posture, 26, 208-13. doi: 10.1016/j.gaitpost.2006.09.010

BIEWENER, A. A. \& ROBERTS, T. J. 2000. Muscle and tendon contributions to force, work, and elastic energy savings: a comparative perspective. Exerc Sport Sci Rev, 28, 99-107.

BIZOVSKA, L., SVOBODA, Z., JANURA, M., BISI, M. C. \& VUILLERME, N. 2018. Local dynamic stability during gait for predicting falls in elderly people: A one-year prospective study. PLoS One, 13, e0197091. doi: 10.1371/journal.pone.0197091

BLAKE, A. J., MORGAN, K., BENDALL, M. J., DALlOSSO, H., EBRAHIM, S. B., ARIE, T. H., FENTEM, P. H. \& BASSEY, E. J. 1988. Falls by elderly people at home: prevalence and associated factors. Age Ageing, 17, 365-72. doi: 10.1093/ageing/17.6.365

BOHM, S., MADEMLI, L., MERSMANN, F. \& ARAMPATZIS, A. 2015. Predictive and Reactive Locomotor Adaptability in Healthy Elderly: A Systematic Review and Meta-Analysis. Sports Med, 45, 1759-77. doi: 10.1007/s40279-015-0413-9

BOSSE, I., OBERLÄNDER, K. D., SAVELBERG, H. H., MEIJER, K., BRÜGGEMANN, G. P. \& KARAMANIDIS, K. 2012. Dynamic stability control in younger and older adults during stair descent. Hum Mov Sci, 31, 1560-70. doi: 10.1016/j.humov.2012.05.003

BOYE, N. D., MATTACE-RASO, F. U., VAN DER VELDE, N., VAN LIESHOUT, E. M., DE VRIES, O. J., HARTHOLT, K. A., KERVER, A. J., BRUIJNINCKX, M. M., VAN DER CAMMEN, T. J., PATKA, P., VAN BEECK, E. F. \& IMPROVEFALL TRIAL COLLABORATORS. 2014. Circumstances leading to injurious falls in older men and women in the Netherlands. Injury, 45, 1224-30. doi: 10.1016/j.injury.2014.03.021

BRANDT, T., STRUPP, M. \& BENSON, J. 1999. You are better off running than walking with acute vestibulopathy. Lancet, 354, 746. doi: 10.1016/S0140-6736(99)03179-7

BRANTBERG, K. \& LOFQVIST, L. 2007. Preserved vestibular evoked myogenic potentials (VEMP) in some patients with walking-induced oscillopsia due to bilateral vestibulopathy. $J$ Vestib Res, 17, 33-8.

BROWN, C. J., GOTTSCHALK, M., VAN NESS, P. H., FORTINSKY, R. H. \& TINETTI, M. E. 2005. Changes in physical therapy providers' use of fall prevention strategies following a multicomponent behavioral change intervention. Phys Ther, 85, 394-403.

BRUIJN, S. M., MEIJER, O. G., BEEK, P. J. \& VAN DIEËN, J. H. 2013. Assessing the stability of human locomotion: a review of current measures. $J R$ Soc Interface, 10, 20120999. doi: 10.1098/rsif.2012.0999

BRUIJN, S. M. \& VAN DIEËN, J. H. 2018. Control of human gait stability through foot placement. $J$ R Soc Interface, 15. doi: 10.1098/rsif.2017.0816

BRUIJN, S. M., VAN DIEËN, J. H. \& DAFFERTSHOFER, A. 2015. Beta activity in the premotor cortex is increased during stabilized as compared to normal walking. Front Hum Neurosci, 9, 593. doi: 10.3389/fnhum.2015.00593

BRUIJN, S. M., VAN IMPE, A., DUYSENS, J. \& SWINNEN, S. P. 2012. Split-belt walking: adaptation differences between young and older adults. J Neurophysiol, 108, 1149-57. doi: $10.1152 / \mathrm{jn} .00018 .2012$ 
BUCZAK-STEC, E. \& GORYNSKI, P. 2013. Fall related hospital admissions among seniors in Poland in 2010. Przegl Epidemiol, 67, 57-62, 141-4.

BURNS, E. \& KAKARA, R. 2018. Deaths from Falls Among Persons Aged $>/=65$ Years - United States, 2007-2016. MMWR Morb Mortal Wkly Rep, 67, 509-514. doi: 10.15585/mmwr.mm6718a1

BURNS, E. R., STEVENS, J. A. \& LEE, R. 2016. The direct costs of fatal and non-fatal falls among older adults - United States. J Safety Res, 58, 99-103. doi: 10.1016/j.jsr.2016.05.001

CALI, C. M. \& KIEL, D. P. 1995. An epidemiologic study of fall-related fractures among institutionalized older people. J Am Geriatr Soc, 43, 1336-40.

CALLISAYA, M. L., SHARMAN, J. E., CLOSE, J., LORD, S. R. \& SRIKANTH, V. K. 2014. Greater daily defined dose of antihypertensive medication increases the risk of falls in older people-a population-based study. J Am Geriatr Soc, 62, 1527-33. doi: 10.1111/jgs.12925

CAMPBELL, A. J., BORRIE, M. J., SPEARS, G. F., JACKSON, S. L., BROWN, J. S. \& FITZGERALD, J. L. 1990. Circumstances and consequences of falls experienced by a community population 70 years and over during a prospective study. Age Ageing, 19, 13641. doi: 10.1093/ageing/19.2.136

CAMPBELL, A. J., REINKEN, J., ALLAN, B. C. \& MARTINEZ, G. S. 1981. Falls in old age: a study of frequency and related clinical factors. Age Ageing, 10, 264-70. doi: 10.1093/ageing/10.4.264

CANNING, C. G., SHERRINGTON, C., LORD, S. R., ClOSE, J. C., HERITIER, S., HELlER, G. Z., HOWARD, K., ALLEN, N. E., LATT, M. D., MURRAY, S. M., O'ROURKE, S. D., PAUL, S. S., SONG, J. \& FUNG, V. S. 2015. Exercise for falls prevention in Parkinson disease: a randomized controlled trial. Neurology, 84, 304-12. doi: 10.1212/WNL.0000000000001155

CARROLL, C. C., DICKINSON, J. M., HAUS, J. M., LEE, G. A., HOLLON, C. J., AAGAARD, P., MAGNUSSON, S. P. \& TRAPPE, T. A. 2008. Influence of aging on the in vivo properties of human patellar tendon. J Appl Physiol, 105, 1907-15. doi: 10.1152/japplphysiol.00059.2008

CARTY, C. P., BARRETT, R. S., CRONIN, N. J., LICHTWARK, G. A. \& MILLS, P. M. 2012a. Lower limb muscle weakness predicts use of a multiple- versus single-step strategy to recover from forward loss of balance in older adults. J Gerontol A Biol Sci Med Sci, 67, 1246-52. doi: 10.1093/gerona/gls149

CARTY, C. P., CRONIN, N. J., LICHTWARK, G. A., MILLS, P. M. \& BARRETT, R. S. 2012b. Lower limb muscle moments and power during recovery from forward loss of balance in male and female single and multiple steppers. Clin Biomech, 27, 1031-7. doi: 10.1016/j.clinbiomech.2012.07.009

CARTY, C. P., CRONIN, N. J., LICHTWARK, G. A., MILLS, P. M. \& BARRETT, R. S. 2012c. Mechanisms of adaptation from a multiple to a single step recovery strategy following repeated exposure to forward loss of balance in older adults. PLoS One, 7, e33591. doi: 10.1371/journal.pone.0033591

CARTY, C. P., CRONIN, N. J., NICHOLSON, D., LICHTWARK, G. A., MILLS, P. M., KERR, G., CRESSWELL, A. G. \& BARRETT, R. S. 2015. Reactive stepping behaviour in response to forward loss of balance predicts future falls in community-dwelling older adults. Age Ageing, 44, 109-15. doi: 10.1093/ageing/afu054

CATTAGNI, T., SCAGLIONI, G., LAROCHE, D., VAN HOECKE, J., GREMEAUX, V. \& MARTIN, A. 2014. Ankle muscle strength discriminates fallers from non-fallers. Front Aging Neurosci, 6, 336. doi: 10.3389/fnagi.2014.00336 
CHAN, K. M., PANG, W. S., EE, C. H., DING, Y. Y. \& CHOO, P. 1997. Epidemiology of falls among the elderly community dwellers in Singapore. Singapore Med J, 38, 427-31.

Chang, J. T., Morton, S. C., RUbenstein, L. Z., MOJiCA, W. A., MAGLione, M., SUTTORP, M. J., ROTH, E. A. \& SHEKELLE, P. G. 2004. Interventions for the prevention of falls in older adults: systematic review and meta-analysis of randomised clinical trials. BMJ, 328, 680. doi: 10.1136/bmj.328.7441.680

CHIEN, J. E. \& HSU, W. L. 2018. Effects of Dynamic Perturbation-Based Training on Balance Control of Community-Dwelling Older Adults. Sci Rep, 8, 17231. doi: 10.1038/s41598-01835644-5

CHIEN, M. H. \& GUO, H. R. 2014. Nutritional status and falls in community-dwelling older people: a longitudinal study of a population-based random sample. PLoS One, 9, e91044. doi: 10.1371/journal.pone.0091044

CHOI, E. J., KIM, S. A., KIM, N. R., RHEE, J. A., YUN, Y. W. \& SHIN, M. H. 2014. Risk factors for falls in older Korean adults: the 2011 Community Health Survey. J Korean Med Sci, 29, 1482-7. doi: 10.3346/jkms.2014.29.11.1482

CHOI, J. T. \& BASTIAN, A. J. 2007. Adaptation reveals independent control networks for human walking. Nat Neurosci, 10, 1055-62. doi: 10.1038/nn1930

CHOU, W. C., TINETTI, M. E., KING, M. B., IRWIN, K. \& FORTINSKY, R. H. 2006. Perceptions of physicians on the barriers and facilitators to integrating fall risk evaluation and management into practice. $J$ Gen Intern Med, 21, 117-22. doi: 10.1111/j.15251497.2005.00298.x

CHU, L. W., CHI, I. \& CHIU, A. Y. 2005. Incidence and predictors of falls in the chinese elderly. Ann Acad Med Singapore, 34, 60-72.

CHU, L. W., CHIU, A. Y. \& CHI, I. 2006. Impact of falls on the balance, gait, and activities of daily living functioning in community-dwelling Chinese older adults. J Gerontol A Biol Sci Med Sci, 61, 399-404. doi: 10.1093/gerona/61.4.399

COUPPE, C., HANSEN, P., KONGSGAARD, M., KOVANEN, V., SUETTA, C., AAGAARD, P., KJAER, M. \& MAGNUSSON, S. P. 2009. Mechanical properties and collagen cross-linking of the patellar tendon in old and young men. J Appl Physiol, 107, 880-6. doi: 10.1152/japplphysiol.00291.2009

COUPPE, C., SUETTA, C., KONGSGAARD, M., JUstesen, L., HVID, L. G., AAGAARD, P., KJAER, M. \& MAGNUSSON, S. P. 2012. The effects of immobilization on the mechanical properties of the patellar tendon in younger and older men. Clin Biomech, 27, 949-54. doi: 10.1016/j.clinbiomech.2012.06.003

COUPPE, C., SVENSSON, R. B., GROSSET, J. F., KOVANEN, V., NIELSEN, R. H., OLSEN, M. R., LARSEN, J. O., PRAET, S. F., SKOVGAARD, D., HANSEN, M., AAGAARD, P., KJAER, M. \& MAGNUSSON, S. P. 2014. Life-long endurance running is associated with reduced glycation and mechanical stress in connective tissue. Age, 36, 9665. doi: 10.1007/s11357014-9665-9

CRAIG, J., MURRAY, A., MITCHELL, S., CLARK, S., SAUNDERS, L. \& BURLEIGH, L. 2013. The high cost to health and social care of managing falls in older adults living in the community in Scotland. Scott Med J, 58, 198-203. doi: 10.1177/0036933013507848

CRENSHAW, J. R., BERNHARDT, K. A., ACHENBACH, S. J., ATKINSON, E. J., KHOSLA, S., KAUFMAN, K. R. \& AMIN, S. 2017. The circumstances, orientations, and impact locations 
of falls in community-dwelling older women. Arch Gerontol Geriatr, 73, 240-247. doi: 10.1016/j.archger.2017.07.011

CRUZ, D. T., RIBEIRO, L. C., VIEIRA MDE, T., TEIXEIRA, M. T., BASTOS, R. R. \& LEITE, I. C. 2012. Prevalence of falls and associated factors in elderly individuals. Rev Saude Publica, 46, 138-46. doi: 10.1590/s0034-89102011005000087

CUMMINGS, S. R., NEVITT, M. C. \& KIDD, S. 1988. Forgetting falls. The limited accuracy of recall of falls in the elderly. J Am Geriatr Soc, 36, 613-6.

DAKIN, C. J., INGLIS, J. T., CHUA, R. \& BLOUIN, J. S. 2013. Muscle-specific modulation of vestibular reflexes with increased locomotor velocity and cadence. J Neurophysiol, 110, 8694. doi: $10.1152 /$ jn. 00843.2012

DAMBREVILLE, C., LABARRE, A., THIBAUDIER, Y., HURTEAU, M. F. \& FRIGON, A. 2015. The spinal control of locomotion and step-to-step variability in left-right symmetry from slow to moderate speeds. J Neurophysiol, 114, 1119-28. doi: 10.1152/jn.00419.2015

DAVIDS, K. W., BUTTON, C. \& BENNETT, S. J. 2008. Dynamics of skill acquisition: A constraintsled approach, Champaign, Human Kinetics.

DAVIES, A. J. \& KENNY, R. A. 1996. Falls presenting to the accident and emergency department: types of presentation and risk factor profile. Age Ageing, 25, 362-6. doi: 10.1093/ageing/25.5.362

DAVIS, J. C., ROBERTSON, M. C., ASHE, M. C., LIU-AMBROSE, T., KHAN, K. M. \& MARRA, C. A. 2010. International comparison of cost of falls in older adults living in the community: a systematic review. Osteoporos Int, 21, 1295-306. doi: 10.1007/s00198-009-1162-0

DECKER, L. M., CIGNETTI, F. \& STERGIOU, N. 2012. Wearing a safety harness during treadmill walking influences lower extremity kinematics mainly through changes in ankle regularity and local stability. J Neuroeng Rehabil, 9, 8. doi: 10.1186/1743-0003-9-8

DE LABRA, C., GUIMARAES-PINHEIRO, C., MASEDA, A., LORENZO, T. \& MILLAN-CALENTI, J. C. 2015. Effects of physical exercise interventions in frail older adults: a systematic review of randomized controlled trials. BMC Geriatr, 15, 154. doi: 10.1186/s12877-015$0155-4$

DE MORTON, N. A. 2009. The PEDro scale is a valid measure of the methodological quality of clinical trials: a demographic study. Aust J Physiother, 55, 129-33.

DEAN, J. C., ALEXANDER, N. B. \& KUO, A. D. 2007. The effect of lateral stabilization on walking in young and old adults. IEEE Trans Biomed Eng, 54, 1919-26. doi: 10.1109/TBME.2007.901031

DEANDREA, S., LUCENTEFORTE, E., BRAVI, F., FOSCHI, R., LA VECCHIA, C. \& NEGRI, E. 2010. Risk factors for falls in community-dwelling older people: a systematic review and meta-analysis. Epidemiology, 21, 658-68. doi: 10.1097/EDE.0b013e3181e89905

Delbaere, K., Close, J. C., Heim, J., SAChDEV, P. S., BRODATY, H., SLAVIN, M. J., KOCHAN, N. A. \& LORD, S. R. 2010. A multifactorial approach to understanding fall risk in older people. J Am Geriatr Soc, 58, 1679-85. doi: 10.1111/j.1532-5415.2010.03017.x

DEMPSTER, W. T., GABEL, W. C. \& FELTS, W. J. 1959. The anthropometry of the manual workspace for the seated subject. Am J Phys Anthropol, 17, 289-317.

DIJKSTRA, B. W., HORAK, F. B., KAMSMA, Y. P. \& PETERSON, D. S. 2015. Older adults can improve compensatory stepping with repeated postural perturbations. Front Aging Neurosci, 7, 201. doi: 10.3389/fnagi.2015.00201 
DING, L. \& YANG, F. 2016. Muscle weakness is related to slip-initiated falls among communitydwelling older adults. J Biomech, 49, 238-43. doi: 10.1016/j.jbiomech.2015.12.009

DINGWELL, J. B., CUSUMANO, J. P., CAVANAGH, P. R. \& STERNAD, D. 2001. Local dynamic stability versus kinematic variability of continuous overground and treadmill walking. $J$ Biomech Eng, 123, 27-32. doi: 10.1115/1.1336798

DO, M. T., CHANG, V. C., KURAN, N. \& THOMPSON, W. 2015. Fall-related injuries among Canadian seniors, 2005-2013: an analysis of the Canadian Community Health Survey. Health Promot Chronic Dis Prev Can, 35, 99-108.

DONALDSON, L. J., COOK, A. \& THOMSON, R. G. 1990. Incidence of fractures in a geographically defined population. J Epidemiol Community Health, 44, 241-5. doi: 10.1136/jech.44.3.241

DONELAN, J. M., SHIPMAN, D. W., KRAM, R. \& KUO, A. D. 2004. Mechanical and metabolic requirements for active lateral stabilization in human walking. J Biomech, 37, 827-35. doi: 10.1016/j.jbiomech.2003.06.002

DOWNTON, J. H. \& ANDREWS, K. 1991. Prevalence, characteristics and factors associated with falls among the elderly living at home. Aging, 3, 219-28.

DU, Y., WOLF, I. K. \& KNOPF, H. 2017. Association of psychotropic drug use with falls among older adults in Germany. Results of the German Health Interview and Examination Survey for Adults 2008-2011 (DEGS1). PLoS One, 12, e0182432. doi: 10.1371/journal.pone.0182432

EKVALL HANSSON, E. \& MAGNUSSON, M. 2013. Vestibular asymmetry predicts falls among elderly patients with multi-sensory dizziness. BMC Geriatr, 13, 77. doi: 10.1186/1471-231813-77

EMKEN, J. L. \& REINKENSMEYER, D. J. 2005. Robot-enhanced motor learning: accelerating internal model formation during locomotion by transient dynamic amplification. IEEE Trans Neural Syst Rehabil Eng, 13, 33-9. doi: 10.1109/TNSRE.2004.843173

EPRO, G., MCCRUM, C., MIERAU, A., LEYENDECKER, M., BRÜGGEMANN, G. P. \& KARAMANIDIS, K. 2018a. Effects of triceps surae muscle strength and tendon stiffness on the reactive dynamic stability and adaptability of older female adults during perturbed walking. J Appl Physiol, 124, 1541-1549. doi: 10.1152/japplphysiol.00545.2017

EPRO, G., MIERAU, A., LEYENDECKER, M., MCCRUM, C., BRÜGGEMANN, G. P. \& KARAMANIDIS, K. Effects of triceps surae muscle strength on gait stability and adaptability in older adults. 11th joint Conference on Motor Control \& Learning, Biomechanics \& Training (German Society of Sports Science), 2016 Darmstadt, Germany.

EPRO, G., MIERAU, A., MCCRUM, C., LEYENDECKER, M., BRÜGGEMANN, G. P. \& KARAMANIDIS, K. 2018b. Retention of gait stability improvements over 1.5 years in older adults: effects of perturbation exposure and triceps surae neuromuscular exercise. $J$ Neurophysiol, 119, 2229-2240. doi: 10.1152/jn.00513.2017

ESPY, D. D., YANG, F., BHATT, T. \& PAI, Y. C. 2010. Independent influence of gait speed and step length on stability and fall risk. Gait Posture, 32, 378-82. doi: 10.1016/j.gaitpost.2010.06.013

FABER, M. J., BOSSCHER, R. J., CHIN, A. P. M. J. \& VAN WIERINGEN, P. C. 2006. Effects of exercise programs on falls and mobility in frail and pre-frail older adults: A multicenter randomized controlled trial. Arch Phys Med Rehabil, 87, 885-96. doi: 10.1016/j.apmr.2006.04.005

FAIRHALL, N., SHERRINGTON, C., LORD, S. R., KURRLE, S. E., LANGRON, C., LOCKWOOD, K., MONAGHAN, N., AGGAR, C. \& CAMERON, I. D. 2014. Effect of a multifactorial, 
interdisciplinary intervention on risk factors for falls and fall rate in frail older people: a randomised controlled trial. Age Ageing, 43, 616-22. doi: 10.1093/ageing/aft204

FELDMAN, F. \& ROBINOVITCH, S. Do judo athletes utilize ukemi falling techniques during unexpected sideways falls? 18th meeting of the International Society for Posture and Gait Research, 2007. 149-150.

FINE, W. 1959. An Analysis of 277 Falls in Hospital. Gerontologia Clinica, 1, 292-300. doi: $10.1159 / 000244584$

FITZPATRICK, R. C., WARDMAN, D. L. \& TAYLOR, J. L. 1999. Effects of galvanic vestibular stimulation during human walking. J Physiol, 517 (Pt 3), 931-9. doi: 10.1111/j.14697793.1999.0931s.x

FORBES, P. A., VLUTTERS, M., DAKIN, C. J., VAN DER KOOIJ, H., BLOUIN, J. S. \& SCHOUTEN, A. C. 2017. Rapid limb-specific modulation of vestibular contributions to ankle muscle activity during locomotion. J Physiol, 595, 2175-2195. doi: 10.1113/JP272614

FORSSBERG, H. 1982. Spinal locomotor functions and descending control. In: SJOLUND, B. \& BJORKLUND, R. (eds.) Brainstem Control of Spinal Mechanisms. Amsterdam, The Netherlands: Elsevier.

FORSSBERG, H., GRILLNER, S. \& ROSSIGNOL, S. 1975. Phase dependent reflex reversal during walking in chronic spinal cats. Brain Res, 85, 103-7. doi: 10.1016/0006-8993(75)91013-6

FORSSBERG, H., GRILLNER, S. \& ROSSIGNOL, S. 1977. Phasic gain control of reflexes from the dorsum of the paw during spinal locomotion. Brain Res, 132, 121-39.

FOTHERGILL, J., O'DRISCOLL, D. \& HASHEMI, K. 1995. The role of environmental factors in causing injury through falls in public places. Ergonomics, 38, 220-223. doi: 10.1080/00140139508925099

FRANCO, M. R., HOWARD, K., SHERRINGTON, C., FERREIRA, P. H., ROSE, J., GOMES, J. L. \& FERREIRA, M. L. 2015. Eliciting older people's preferences for exercise programs: a bestworst scaling choice experiment. J Physiother, 61, 34-41. doi: 10.1016/j.jphys.2014.11.001

FRANCO, M. R., HOWARD, K., SHERRINGTON, C., ROSE, J., FERREIRA, P. H. \& FERREIRA, M. L. 2016. Smallest worthwhile effect of exercise programs to prevent falls among older people: estimates from benefit-harm trade-off and discrete choice methods. Age Ageing, 45, 806-812. doi: 10.1093/ageing/afw110

FREYLER, K., KRAUSE, A., GOLLHOFER, A. \& RITZMANN, R. 2016. Specific Stimuli Induce Specific Adaptations: Sensorimotor Training vs. Reactive Balance Training. PLoS One, 11, e0167557. doi: 10.1371/journal.pone.0167557

FRIGON, A. 2017. The neural control of interlimb coordination during mammalian locomotion. $J$ Neurophysiol, 117, 2224-2241. doi: 10.1152/jn.00978.2016

FRIGON, A. \& ROSSIGNOL, S. 2008. Adaptive changes of the locomotor pattern and cutaneous reflexes during locomotion studied in the same cats before and after spinalization. $J$ Physiol, 586, 2927-45. doi: 10.1113/jphysiol.2008.152488

FUJIMOTO, C., MUROFUSHI, T., CHIHARA, Y., SUZUKI, M., YAMASOBA, T. \& IWASAKI, S. 2009. Novel subtype of idiopathic bilateral vestibulopathy: bilateral absence of vestibular evoked myogenic potentials in the presence of normal caloric responses. $J$ Neurol, 256, 1488-92. doi: 10.1007/s00415-009-5147-x

FUJIMOTO, M., BAIR, W. N. \& ROGERS, M. W. 2015. Center of pressure control for balance maintenance during lateral waist-pull perturbations in older adults. $J$ Biomech, 48, 963-8. doi: 10.1016/j.jbiomech.2015.02.012 
GADELHA, A. B., NERI, S. G. R., BOTTARO, M. \& LIMA, R. M. 2018a. The relationship between muscle quality and incidence of falls in older community-dwelling women: An 18-month follow-up study. Exp Gerontol, 110, 241-246. doi: 10.1016/j.exger.2018.06.018

GADELHA, A. B., NERI, S. G. R., NOBREGA, O. T., PEREIRA, J. C., BOTTARO, M., FONSECA, A. \& LIMA, R. M. 2018b. Muscle quality is associated with dynamic balance, fear of falling, and falls in older women. Exp Gerontol, 104, 1-6. doi: 10.1016/j.exger.2018.01.003

GALE, C. R., COOPER, C. \& AIHIE SAYER, A. 2016. Prevalence and risk factors for falls in older men and women: The English Longitudinal Study of Ageing. Age Ageing, 45, 789-794. doi: 10.1093/ageing/afw129

GATES, D. H., DARTER, B. J., DINGWELL, J. B. \& WILKEN, J. M. 2012. Comparison of walking overground and in a Computer Assisted Rehabilitation Environment (CAREN) in individuals with and without transtibial amputation. $J$ Neuroeng Rehabil, 9, 81. doi: 10.1186/1743-0003-9-81

GEERSE, D. J., ROERDINK, M., MARINUS, J. \& VAN HILTEN, J. J. 2019. Walking adaptability for targeted fall-risk assessments. Gait Posture, 70, 203-210. doi: 10.1016/j.gaitpost.2019.02.013

GERARDS, M. H. G., MCCRUM, C., MANSFIELD, A. \& MEIJER, K. 2017. Perturbation-based balance training for falls reduction among older adults: Current evidence and implications for clinical practice. Geriatr Gerontol Int, 17, 2294-2303. doi: 10.1111/ggi.13082

GERVASIO, S., KERSTING, U. G., FARINA, D. \& MRACHACZ-KERSTING, N. 2015. The effect of crossed reflex responses on dynamic stability during locomotion. J Neurophysiol, 114, 1034-40. doi: 10.1152/jn.00178.2015

GHODSI, S. M., ROUDSARI, B. S., ABDOLLAHI, M. \& SHADMAN, M. 2003. Fall-related injuries in the elderly in Tehran. Injury, 34, 809-14.

GILASI, H. R., SOORI, H., YAZDANI, S. \& TAHERI TENJANI, P. 2015. Fall-Related Injuries in Community-Dwelling Older Adults in Qom Province, Iran, 2010-2012. Arch Trauma Res, 4, e22925. doi: 10.5812/atr.22925

GILL, T., TAYLOR, A. W. \& PENGELLY, A. 2005. A population-based survey of factors relating to the prevalence of falls in older people. Gerontology, 51, 340-5. doi: 10.1159/000086372

GILlesPiE, L. D., ROBERTSON, M. C., GILlESPIE, W. J., SHERRINGTON, C., GATES, S., CLEMSON, L. M. \& LAMB, S. E. 2012. Interventions for preventing falls in older people living in the community. Cochrane Database Syst Rev, 9, CD007146. doi: 10.1002/14651858.CD007146.pub3

GOLDBERG, J. M., WILSON, V. J., CULlEN, K. E., ANGELAKI, D. E., BROUSSARD, D. M., BUTTNER-ENNEVER, J., FUKUSHIMA, K. \& MINOR, L. B. 2012. The Vestibular System, OUP USA.

GOVENDER, S., ROSENGREN, S. M. \& COLEBATCH, J. G. 2011. Vestibular neuritis has selective effects on air- and bone-conducted cervical and ocular vestibular evoked myogenic potentials. Clin Neurophysiol, 122, 1246-55. doi: 10.1016/j.clinph.2010.12.040

GRABINER, M. D., BAREITHER, M. L., GATTS, S., MARONE, J. \& TROY, K. L. 2012. Taskspecific training reduces trip-related fall risk in women. Med Sci Sports Exerc, 44, 2410-4. doi: 10.1249/MSS.0b013e318268c89f

GRABINER, M. D., CRENSHAW, J. R., HURT, C. P., ROSENBLATT, N. J. \& TROY, K. L. 2014. Exercise-based fall prevention: can you be a bit more specific? Exerc Sport Sci Rev, 42, 1618. doi: 10.1249/JES.0000000000000023 
GRABINER, M. D., DONOVAN, S., BAREITHER, M. L., MARONE, J. R., HAMSTRA-WRIGHT, K., GATTS, S. \& TROY, K. L. 2008. Trunk kinematics and fall risk of older adults: translating biomechanical results to the clinic. J Electromyogr Kinesiol, 18, 197-204. doi: 10.1016/j.jelekin.2007.06.009

GRABINER, M. D., OWINGS, T. M. \& PAVOL, M. J. 2005. Lower extremity strength plays only a small role in determining the maximum recoverable lean angle in older adults. J Gerontol A Biol Sci Med Sci, 60, 1447-50. doi: 10.1093/gerona/60.11.1447

GRAHAM, D. F., CARTY, C. P., LLOYD, D. G. \& BARRETT, R. S. 2015. Biomechanical predictors of maximal balance recovery performance amongst community-dwelling older adults. Exp Gerontol, 66, 39-46. doi: 10.1016/j.exger.2015.04.006

GRANACHER, U., MUEHLBAUER, T., ZAHNER, L., GOLLHOFER, A. \& KRESSIG, R. W. 2011. Comparison of traditional and recent approaches in the promotion of balance and strength in older adults. Sports Med, 41, 377-400. doi: 10.2165/11539920-000000000-00000

GRANATA, K. P. \& ENGLAND, S. A. 2007. Reply to the Letter to the Editor. Gait Posture, 26, 329330. doi: 10.1016/j.gaitpost.2007.02.002

GRANT, A., MACKENZIE, L. \& CLEMSON, L. 2015. How do general practitioners engage with allied health practitioners to prevent falls in older people? An exploratory qualitative study. Australas J Ageing, 34, 149-54. doi: 10.1111/ajag.12157

GRIFFIN, J., LALL, R., BRUCE, J., WITHERS, E., FINNEGAN, S., LAMB, S. E. \& PRE, F. I. T. S. G. 2019. Comparison of alternative falls data collection methods in the Prevention of Falls Injury Trial (PreFIT). J Clin Epidemiol, 106, 32-40. doi: 10.1016/j.jclinepi.2018.09.006

GRILL, E., HEUBERGER, M., STROBL, R., SAGLAM, M., HOLLE, R., LINKOHR, B., LADWIG, K. H., PETERS, A., SCHNEIDER, E., JAHN, K. \& LEHNEN, N. 2018. Prevalence, Determinants, and Consequences of Vestibular Hypofunction. Results From the KORA-FF4 Survey. Front Neurol, 9, 1076. doi: 10.3389/fneur.2018.01076

GRISSO, J. A., SCHWARZ, D. F., WOLFSON, V., POLANSKY, M. \& LAPANN, K. 1992. The impact of falls in an inner-city elderly African-American population. J Am Geriatr Soc, 40, 673-8.

GROEN, B. E., SMULDERS, E., DE KAM, D., DUYSENS, J. \& WEERDESTEYN, V. 2010a. Martial arts fall training to prevent hip fractures in the elderly. Osteoporos Int, 21, 215-21. doi: 10.1007/s00198-009-0934-x

GROEN, B. E., SMULDERS, E., DUYSENS, J., VAN LANKVELD, W. \& WEERDESTEYN, V. 2010b. Could martial arts fall training be safe for persons with osteoporosis?: a feasibility study. BMC Res Notes, 3, 111. doi: 10.1186/1756-0500-3-111

GUERIN, B., HOORENS, S., KHODYAKOV, D. \& YAQUB, O. 2015. A growing and ageing population-global societal trends to 2030: thematic report 1. Research Reports. RR-920/1EC. Santa Monica, CA, RAND Corporation.

GUINAND, N., BOSELIE, F., GUYOT, J. P. \& KINGMA, H. 2012a. Quality of life of patients with bilateral vestibulopathy. Ann Otol Rhinol Laryngol, 121, 471-7. doi: 10.1177/000348941212100708

GUINAND, N., PIJNENBURG, M., JANSSEN, M. \& KINGMA, H. 2012b. Visual Acuity While Walking and Oscillopsia Severity in Healthy Subjects and Patients With Unilateral and Bilateral Vestibular Function Loss. Arch Otolaryngol Head Neck Surg, 138, 301-306.

GUINAND, N., VAN DE BERG, R., CAVUSCENS, S., STOKROOS, R. J., RANIERI, M., PELIZZONE, M., KINGMA, H., GUYOT, J. P. \& PEREZ-FORNOS, A. 2015. Vestibular 
Implants: 8 Years of Experience with Electrical Stimulation of the Vestibular Nerve in 11 Patients with Bilateral Vestibular Loss. ORL J Otorhinolaryngol Relat Spec, 77, 227-240. doi: 10.1159/000433554

GUYOT, J. P., PEREZ FORNOS, A., GUINAND, N., VAN DE BERG, R., STOKROOS, R. \& KINGMA, H. 2016. Vestibular assistance systems: promises and challenges. J Neurol, 263 Suppl 1, S30-5. doi: 10.1007/s00415-015-7922-1

HAK, L., HOUDIJK, H., BEEK, P. J. \& VAN DIEËN, J. H. 2013. Steps to take to enhance gait stability: the effect of stride frequency, stride length, and walking speed on local dynamic stability and margins of stability. PLoS One, 8, e82842. doi: 10.1371/journal.pone.0082842

HAK, L., HOUDIJK, H., STEENBRINK, F., MERT, A., VAN DER WURFF, P., BEEK, P. J. \& VAN DIEËN, J. H. 2012. Speeding up or slowing down?: Gait adaptations to preserve gait stability in response to balance perturbations. Gait Posture, 36, 260-4. doi: 10.1016/j.gaitpost.2012.03.005

HAKEN, H. 1987. Synergetics: An approach to self-organization. Self-organizing systems: The emergence of order. New York: Plenum Press.

HALIL, M., Ulger, Z., CANKURTARAN, M., Shorbagi, A., YAVUZ, B. B., DEDE, D., OZKAYAR, N. \& ARIOGUL, S. 2006. Falls and the elderly: is there any difference in the developing world? A cross-sectional study from Turkey. Arch Gerontol Geriatr, 43, 351-9. doi: 10.1016/j.archger.2005.12.005

HALL, S. E. \& HENDRIE, D. V. 2003. A prospective study of the costs of falls in older adults living in the community. Aust N Z J Public Health, 27, 343-51.

HAMED, A., BOHM, S., MERSMANN, F. \& ARAMPATZIS, A. 2018. Follow-up efficacy of physical exercise interventions on fall incidence and fall risk in healthy older adults: a systematic review and meta-analysis. Sports Med Open, 4, 56. doi: 10.1186/s40798-018-0170-z

HARIDAS, C., ZEHR, E. P. \& MISIASZEK, J. E. 2008. Adaptation of cutaneous stumble correction when tripping is part of the locomotor environment. $J$ Neurophysiol, 99, 2789-97. doi: 10.1152/jn.00487.2007

HARPER, S. \& WALPORT, M. 2016. Future of an ageing population. London: Government Office for Science. GS/16/10

HARThOLT, K. A., POLINDER, S., VAN DER CAMMEN, T. J., PANNEMAN, M. J., VAN DER VELDE, N., VAN LIESHOUT, E. M., PATKA, P. \& VAN BEECK, E. F. 2012. Costs of falls in an ageing population: a nationwide study from the Netherlands (2007-2009). Injury, 43, 1199-203. doi: 10.1016/j.injury.2012.03.033

HARTHOLT, K. A., VAN BEECK, E. F., POLINDER, S., VAN DER VELDE, N., VAN LIESHOUT, E. M., PANNEMAN, M. J., VAN DER CAMMEN, T. J. \& PATKA, P. 2011. Societal consequences of falls in the older population: injuries, healthcare costs, and long-term reduced quality of life. J Trauma, 71, 748-53. doi: 10.1097/TA.0b013e3181f6f5e5

HARTHOLT, K. A., VAN DER VELDE, N., LOOMAN, C. W., VAN LIESHOUT, E. M., PANNEMAN, M. J., VAN BEECK, E. F., PATKA, P. \& VAN DER CAMMEN, T. J. 2010. Trends in fall-related hospital admissions in older persons in the Netherlands. Arch Intern Med, 170, 905-11. doi: 10.1001/archinternmed.2010.106

HAUSDORFF, J. M., RIOS, D. A. \& EDELBERG, H. K. 2001. Gait variability and fall risk in community-living older adults: a 1-year prospective study. Arch Phys Med Rehabil, 82, 1050-6. doi: 10.1053/apmr.2001.24893 
HAVENS, K. L., MUKHERJEE, T. \& FINLEY, J. M. 2018. Analysis of biases in dynamic margins of stability introduced by the use of simplified center of mass estimates during walking and turning. Gait Posture, 59, 162-167. doi: 10.1016/j.gaitpost.2017.10.002

HEIDEN, T. L., SANDERSON, D. J., INGLIS, J. T. \& SIEGMUND, G. P. 2006. Adaptations to normal human gait on potentially slippery surfaces: the effects of awareness and prior slip experience. Gait Posture, 24, 237-46. doi: 10.1016/j.gaitpost.2005.09.004

HERDMAN, S. J., BLATT, P., SCHUBERT, M. C. \& TUSA, R. J. 2000. Falls in patients with vestibular deficits. Am J Otol, 21, 847-51.

HERSSENS, N. \& MCCRUM, C. 2019. Stimulating balance: recent advances in vestibular stimulation for balance and gait. J Neurophysiol. doi: 10.1152/jn.00851.2018

HERSSENS, N., VERBECQUE, E., HALLEMANS, A., VEREECK, L., VAN ROMPAEY, V. \& SAEYS, W. 2018. Do spatiotemporal parameters and gait variability differ across the lifespan of healthy adults? A systematic review. Gait Posture, 64, 181-190. doi: 10.1016/j.gaitpost.2018.06.012

HILL, K., SCHWARZ, J., FLICKER, L. \& CARROLL, S. 1999. Falls among healthy, communitydwelling, older women: a prospective study of frequency, circumstances, consequences and prediction accuracy. Aust NZ J Public Health, 23, 41-8.

HILL, K. D., DAY, L. \& HAINES, T. P. 2014. What factors influence community-dwelling older people's intent to undertake multifactorial fall prevention programs? Clin Interv Aging, 9, 2045-53. doi: 10.2147/CIA.S72679

HILLIARD, M. J., MARTINEZ, K. M., JANSSEN, I., EDWARDS, B., MILLE, M. L., ZHANG, Y. \& ROGERS, M. W. 2008. Lateral balance factors predict future falls in community-living older adults. Arch Phys Med Rehabil, 89, 1708-13. doi: 10.1016/j.apmr.2008.01.023

HOF, A. L. 1996. Scaling gait data to body size. Gait Posture, 4, 222-223. doi: 10.1016/09666362(95)01057-2

HOF, A. L. 2007. The equations of motion for a standing human reveal three mechanisms for balance. J Biomech, 40, 451-7. doi: 10.1016/j.jbiomech.2005.12.016

HOF, A. L. 2008. The 'extrapolated center of mass' concept suggests a simple control of balance in walking. Hum Mov Sci, 27, 112-25. doi: 10.1016/j.humov.2007.08.003

HOF, A. L., GAZENDAM, M. G. \& SINKE, W. E. 2005. The condition for dynamic stability. $J$ Biomech, 38, 1-8. doi: 10.1016/j.jbiomech.2004.03.025

HOF, A. L., VAN ZANDWIJK, J. P. \& BOBBERT, M. F. 2002. Mechanics of human triceps surae muscle in walking, running and jumping. Acta Physiol Scand, 174, 17-30. doi: 10.1046/j.1365-201x.2002.00917.x

HOF, A. L., VERMERRIS, S. M. \& GJALTEMA, W. A. 2010. Balance responses to lateral perturbations in human treadmill walking. $J$ Exp Biol, 213, 2655-64. doi: 10.1242/jeb.042572

HOLLMAN, J. H., BREY, R. H., ROBB, R. A., BANG, T. J. \& KAUFMAN, K. R. 2006. Spatiotemporal gait deviations in a virtual reality environment. Gait Posture, 23, 441-4. doi: 10.1016/j.gaitpost.2005.05.005

HOLlMAN, J. H., CHILDS, K. B., MCNEIL, M. L., MUELlER, A. C., QUILTER, C. M. \& YOUDAS, J. W. 2010. Number of strides required for reliable measurements of pace, rhythm and variability parameters of gait during normal and dual task walking in older individuals. Gait Posture, 32, 23-8. doi: 10.1016/j.gaitpost.2010.02.017 
HOLLMAN, J. H., MCDADE, E. M. \& PETERSEN, R. C. 2011. Normative spatiotemporal gait parameters in older adults. Gait Posture, 34, 111-8. doi: 10.1016/j.gaitpost.2011.03.024

HOMANN, B., PLASCHG, A., GRUNDNER, M., HAUBENHOFER, A., GRIEDL, T., IVANIC, G., HOFER, E., FAZEKAS, F. \& HOMANN, C. N. 2013. The impact of neurological disorders on the risk for falls in the community dwelling elderly: a case-controlled study. BMJ Open, 3, e003367. doi: 10.1136/bmjopen-2013-003367

HONG, I., SIMPSON, A. N., LOGAN, S. \& WOO, H. S. 2016. Longitudinal Trends in Fall Accidents in Community Dwelling Korean Adults: The 2008-2013 Korean Community Health Survey. Ann Rehabil Med, 40, 657-65. doi: 10.5535/arm.2016.40.4.657

HOOGKAMER, W., MASSAAD, F., JANSEN, K., BRUIJN, S. M. \& DUYSENS, J. 2012. Selective bilateral activation of leg muscles after cutaneous nerve stimulation during backward walking. J Neurophysiol, 108, 1933-41. doi: 10.1152/jn.01159.2011

HORAK, F. B., KLUZIK, J. \& HLAVACKA, F. 2016. Velocity dependence of vestibular information for postural control on tilting surfaces. $J$ Neurophysiol, 116, 1468-79. doi: 10.1152/jn.00057.2016

HOULDIN, A., CHUA, R., CARPENTER, M. G. \& LAM, T. 2012. Limited interlimb transfer of locomotor adaptations to a velocity-dependent force field during unipedal walking. $J$ Neurophysiol, 108, 943-52. doi: 10.1152/jn.00670.2011

HOWELL, T. H. 1958. Analysis of falls in old people. J Am Geriatr Soc, 6, 522-5.

HOWLAND, J., HACKMAN, H., TAYLOR, A., O'HARA, K., LIU, J. \& BRUSCH, J. 2018. Older adult fall prevention practices among primary care providers at accountable care organizations: A pilot study. PLoS One, 13, e0205279. doi: 10.1371/journal.pone.0205279

HSIAO, M. Y., CHEN, Y. C., LIN, C. Y., CHEN, W. S. \& WANG, T. G. 2015. Reduced Patellar Tendon Elasticity with Aging: In Vivo Assessment by Shear Wave Elastography. Ultrasound Med Biol, 41, 2899-905. doi: 10.1016/j.ultrasmedbio.2015.07.008

HUANG, T. W., SHORTER, K. A., ADAMCZYK, P. G. \& KUO, A. D. 2015. Mechanical and energetic consequences of reduced ankle plantar-flexion in human walking. J Exp Biol, 218, 3541-50. doi: $10.1242 /$ jeb.113910

HUNTLEY, A. H., SCHINKEL-IVY, A., AQUI, A. \& MANSFIELD, A. 2017. Validation of simplified centre of mass models during gait in individuals with chronic stroke. Clin Biomech, 48, 97102. doi: 10.1016/j.clinbiomech.2017.07.015

HUSSAIN, S. J. \& MORTON, S. M. 2014. Perturbation schedule does not alter retention of a locomotor adaptation across days. J Neurophysiol, 111, 2414-22. doi: 10.1152/jn.00570.2013

IWASAKI, S. \& YAMASOBA, T. 2015. Dizziness and Imbalance in the Elderly: Age-related Decline in the Vestibular System. Aging Dis, 6, 38-47. doi: 10.14336/AD.2014.0128

JACK, C. I., SMITH, T., NEOH, C., LYE, M. \& MCGALLIARD, J. N. 1995. Prevalence of low vision in elderly patients admitted to an acute geriatric unit in Liverpool: elderly people who fall are more likely to have low vision. Gerontology, 41, 280-5. doi: 10.1159/000213695

JAHN, K., DEUTSCHLANDER, A., STEPHAN, T., KALlA, R., HUFNER, K., WAGNER, J., STRUPP, M. \& BRANDT, T. 2008. Supraspinal locomotor control in quadrupeds and humans. Prog Brain Res, 171, 353-62. doi: 10.1016/S0079-6123(08)00652-3

JAHN, K., STRUPP, M., SCHNEIDER, E., DIETERICH, M. \& BRANDT, T. 2000. Differential effects of vestibular stimulation on walking and running. Neuroreport, 11, 1745-8. 
JAHN, K., STRUPP, M., SCHNEIDER, E., DIETERICH, M. \& BRANDT, T. 2001. Visually induced gait deviations during different locomotion speeds. Exp Brain Res, 141, 370-4. doi: $10.1007 / \mathrm{s} 002210100884$

JI, L. \& ZHAI, S. 2018. Aging and the peripheral vestibular system. J Otol, 13, 138-140. doi: 10.1016/j.joto.2018.11.006

JOBGES, M., HEUSCHKEL, G., PRETZEL, C., ILLHARDT, C., RENNER, C. \& HUMMELSHEIM, H. 2004. Repetitive training of compensatory steps: a therapeutic approach for postural instability in Parkinson's disease. J Neurol Neurosurg Psychiatry, 75, 1682-7. doi: 10.1136/jnnp.2003.016550

JONES, T. S., GHOSH, T. S., HORN, K., SMITH, J. \& VOGT, R. L. 2011. Primary care physicians perceptions and practices regarding fall prevention in adult's 65 years and over. Accid Anal Prev, 43, 1605-9. doi: 10.1016/j.aap.2011.03.013

KALULA, S. Z., FERREIRA, M., SWINGLER, G. H. \& BADRI, M. 2016. Risk factors for falls in older adults in a South African Urban Community. BMC Geriatr, 16, 51. doi: 10.1186/s12877-016-0212-7

KALULA, S. Z., SCOTT, V., DOWD, A. \& BRODRICK, K. 2011. Falls and fall prevention programmes in developing countries: environmental scan for the adaptation of the Canadian Falls prevention curriculum for developing countries. J Safety Res, 42, 461-72. doi: 10.1016/j.jsr.2010.12.003

KAMEL, M. H., ABDULMAJEED, A. A. \& ISMAIL SEL, S. 2013. Risk factors of falls among elderly living in urban Suez--Egypt. Pan Afr Med J, 14, 26. doi: 10.11604/pamj.2013.14.26.1609

KANG, L., HAN, P., WANG, J., MA, Y., JIA, L., FU, L., YU, H., CHEN, X., NIU, K. \& GUO, Q. 2017. Timed Up and Go Test can predict recurrent falls: a longitudinal study of the community-dwelling elderly in China. Clin Interv Aging, 12, 2009-2016. doi: 10.2147/CIA.S138287

KANNUS, P., NIEMI, S., PALVANEN, M. \& PARKKARI, J. 2000. Continuously increasing number and incidence of fall-induced, fracture-associated, spinal cord injuries in elderly persons. Arch Intern Med, 160, 2145-9.

KANNUS, P., NIEMI, S., PARKKARI, J., PALVANEN, M. \& SIEVANEN, H. 2007a. Alarming rise in fall-induced severe head injuries among elderly people. Injury, 38, 81-3. doi: 10.1016/j.injury.2006.08.027

KANNUS, P., PALVANEN, M., NIEMI, S. \& PARKKARI, J. 2007b. Alarming rise in the number and incidence of fall-induced cervical spine injuries among older adults. J Gerontol A Biol Sci Med Sci, 62, 180-3. doi: 10.1093/gerona/62.2.180

KAO, P. C., SRIVASTAVA, S., AGRAWAL, S. K. \& SCHOLZ, J. P. 2013. Effect of robotic performance-based error-augmentation versus error-reduction training on the gait of healthy individuals. Gait Posture, 37, 113-20. doi: 10.1016/j.gaitpost.2012.06.025

KARAMANIDIS, K. \& ARAMPATZIS, A. 2006. Mechanical and morphological properties of human quadriceps femoris and triceps surae muscle-tendon unit in relation to aging and running. J Biomech, 39, 406-17. doi: 10.1016/j.jbiomech.2004.12.017

KARAMANIDIS, K. \& ARAMPATZIS, A. 2007. Age-related degeneration in leg-extensor muscletendon units decreases recovery performance after a forward fall: compensation with running experience. Eur J Appl Physiol, 99, 73-85. doi: 10.1007/s00421-006-0318-2 
KARAMANIDIS, K., ARAMPATZIS, A. \& MADEMLI, L. 2008. Age-related deficit in dynamic stability control after forward falls is affected by muscle strength and tendon stiffness. $J$ Electromyogr Kinesiol, 18, 980-9. doi: 10.1016/j.jelekin.2007.04.003

KARAMANIDIS, K., EPRO, G., MCCRUM, C. \& KÖNIG, M. (2019). Improving trip and slipresisting skills in older people: perturbation dose matters. Exerc Sport Sci Rev. doi: 10.1249/JES.0000000000000210

KAREKLA, X. \& TYLER, N. 2018a. Maintaining balance on a moving bus: The importance of threepeak steps whilst climbing stairs. Transp Res Part A Policy Pract, 116, 339-349. doi: 10.1016/j.tra.2018.06.020

KAREKLA, X. \& TYLER, N. 2018b. Maintaining balance on a moving bus: The importance of threepeak steps whilst walking on the lower-deck. Transp Res Part A Policy Pract, 116, 484-496. doi: 10.1016/j.tra.2018.06.022

KARIO, K., TOBIN, J. N., WOLFSON, L. I., WHIPPLE, R., DERBY, C. A., SINGH, D., MARANTZ, P. R. \& WASSERTHEIL-SMOLLER, S. 2001. Lower standing systolic blood pressure as a predictor of falls in the elderly: a community-based prospective study. J Am Coll Cardiol, $38,246-52$.

KATSAVELIS, D., MUKHERJEE, M., DECKER, L. \& STERGIOU, N. 2010. The effect of virtual reality on gait variability. Nonlinear Dynamics Psychol Life Sci, 14, 239-56.

KELSEY, J. L., PROCTER-GRAY, E., HANNAN, M. T. \& LI, W. 2012. Heterogeneity of falls among older adults: implications for public health prevention. Am J Public Health, 102, 2149-56. doi: 10.2105/AJPH.2012.300677

KIAMI, S. R., SKY, R. \& GOODGOLD, S. 2019. Facilitators and barriers to enrolling in falls prevention programming among community dwelling older adults. Arch Gerontol Geriatr, 82, 106-113. doi: 10.1016/j.archger.2019.01.006

KIELICH, K., MACKENZIE, L., LOVARINI, M. \& CLEMSON, L. 2017. Urban Australian general practitioners' perceptions of falls risk screening, falls risk assessment, and referral practices for falls prevention: an exploratory cross-sectional survey study. Aust Health Rev, 41, 111-119. doi: 10.1071/AH15152

KIM, J. C., CHON, J., KIM, H. S., LEE, J. H., YOO, S. D., KIM, D. H., LEE, S. A., HAN, Y. J., LEE, H. S., LEE, B. Y., SOH, Y. S. \& WON, C. W. 2017. The Association Between Fall History and Physical Performance Tests in the Community-Dwelling Elderly: A Cross-Sectional Analysis. Ann Rehabil Med, 41, 239-247. doi: 10.5535/arm.2017.41.2.239

KING, M. B. 2009. Falls. In: HALTER, J. B., OUSLANDER, J. G., TINETTI , M. E., STUDENSKI, S., HIGH, K. P. \& ASTHANA, S. A. (eds.) Hazzard's Geriatric Medicine and Gerontology. 6th ed. USA: McGraw-Hill.

KINGMA, H., FELIPE, L., GERARDS, M. C., GERITS, P., GUINAND, N., PEREZ-FORNOS, A., DEMKIN, V. \& VAN DE BERG, R. 2018. Vibrotactile feedback improves balance and mobility in patients with severe bilateral vestibular loss. $J$ Neurol. doi: 10.1007/s00415018-9133-z

KINGMA, H. \& VAN DE BERG, R. 2016. Chapter 1 - Anatomy, physiology, and physics of the peripheral vestibular system. In: JOSEPH, M. F. \& THOMAS, L. (eds.) Handbook of Clinical Neurology. Elsevier. doi: 10.1016/B978-0-444-63437-5.00001-7

KINSELLA, K., SUZMAN, R., ROBINE, J. M. \& MYER, G. 2000. Demography of older populations in developed countries. In: EVANS, J. G., WILLIAMS, T. F., BEATTIE, B. L., MICHEL, J. 
P. \& WILCOCK, G. K. (eds.) Oxford Textbook of Geriatric Medicine. 2nd ed. Oxford, UK: Oxford University Press.

KISS, R., SCHEDLER, S. \& MUEHLBAUER, T. 2018. Associations Between Types of Balance Performance in Healthy Individuals Across the Lifespan: A Systematic Review and MetaAnalysis. Front Physiol, 9, 1366. doi: 10.3389/fphys.2018.01366

KOJIMA, G., KENDRICK, D., SKELTON, D. A., MORRIS, R. W., GAWLER, S. \& ILIFFE, S. 2015. Frailty predicts short-term incidence of future falls among British community-dwelling older people: a prospective cohort study nested within a randomised controlled trial. BMC Geriatr, 15, 155. doi: 10.1186/s12877-015-0152-7

KOJIMA, S., FURUNA, T., IKEDA, N., NAKAMURA, M. \& SAWADA, Y. 2008. Falls among community-dwelling elderly people of Hokkaido, Japan. Geriatr Gerontol Int, 8, 272-7. doi: 10.1111/j.1447-0594.2008.00476.x

KONIG, N., SINGH, N. B., VON BECKERATH, J., JANKE, L. \& TAYLOR, W. R. 2014. Is gait variability reliable? An assessment of spatio-temporal parameters of gait variability during continuous overground walking. Gait Posture, 39, 615-7. doi: 10.1016/j.gaitpost.2013.06.014

KÖNIG, M., EPRO, G., SEELEY, J., CATALA-LEHNEN, P., POTTHAST, W. \& KARAMANIDIS, K. 2019. Retention of improvement in gait stability over 14 weeks due to trip-perturbation training is dependent on perturbation dose. $J$ Biomech, 84, 243-246. doi: 10.1016/j.jbiomech.2018.12.011

KOSOROK, M. R., OMENN, G. S., DIEHR, P., KOEPSELL, T. D. \& PATRICK, D. L. 1992. Restricted activity days among older adults. Am J Public Health, 82, 1263-7. doi: 10.2105/ajph.82.9.1263

KRASOVSKY, T., LAMONTAGNE, A., FELDMAN, A. G. \& LEVIN, M. F. 2014. Effects of walking speed on gait stability and interlimb coordination in younger and older adults. Gait Posture, 39, 378-85. doi: 10.1016/j.gaitpost.2013.08.011

KRAUSE, A., FREYlER, K., GOLlHOFER, A., STOCKER, T., BRUDERLIN, U., COLIN, R., TOPFER, H. \& RITZMANN, R. 2018. Neuromuscular and Kinematic Adaptation in Response to Reactive Balance Training - a Randomized Controlled Study Regarding Fall Prevention. Front Physiol, 9, 1075. doi: 10.3389/fphys.2018.01075

KUMMEL, J., KRAMER, A., GIBOIN, L. S. \& GRUBER, M. 2016. Specificity of Balance Training in Healthy Individuals: A Systematic Review and Meta-Analysis. Sports Med, 46, 1261-71. doi: $10.1007 / \mathrm{s} 40279-016-0515-\mathrm{z}$

KUO, A. D. 2007. The six determinants of gait and the inverted pendulum analogy: A dynamic walking perspective. Hum Mov Sci, 26, 617-56. doi: 10.1016/j.humov.2007.04.003

KUO, A. D., DONELAN, J. M. \& RUINA, A. 2005. Energetic consequences of walking like an inverted pendulum: step-to-step transitions. Exerc Sport Sci Rev, 33, 88-97.

KURZ, I., GIMMON, Y., SHAPIRO, A., DEBI, R., SNIR, Y. \& MELZER, I. 2016. Unexpected perturbations training improves balance control and voluntary stepping times in older adults - a double blind randomized control trial. BMC Geriatr, 16, 58. doi: 10.1186/s12877016-0223-4

KWAN, E., STRAUS, S. \& HOLROYD-LEDUC, J. 2016. Risk Factors for Falls in the Elderly. In: HUANG, A. R. \& MALLET, L. (eds.) Medication-Related Falls in Older People. Switzerland: Springer International Publishing. doi: 10.1007/978-3-319-32304-6_8

LAM, T., ANDERSCHITZ, M. \& DIETZ, V. 2006. Contribution of feedback and feedforward strategies to locomotor adaptations. J Neurophysiol, 95, 766-73. doi: 10.1152/jn.00473.2005 
LAM, T., WOLSTENHOLME, C., VAN DER LINDEN, M., PANG, M. Y. \& YANG, J. F. 2003. Stumbling corrective responses during treadmill-elicited stepping in human infants. $J$ Physiol, 553, 319-31. doi: 10.1113/jphysiol.2003.043984

LAMB, S. E., JORSTAD-STEIN, E. C., HAUER, K., BECKER, C., PREVENTION OF FALLS NETWORK, E. \& OUTCOMES CONSENSUS, G. 2005. Development of a common outcome data set for fall injury prevention trials: the Prevention of Falls Network Europe consensus. J Am Geriatr Soc, 53, 1618-22. doi: 10.1111/j.1532-5415.2005.53455.x

LARSSON, J., EKVALL HANSSON, E. \& MILLER, M. 2015. Increased double support variability in elderly female fallers with vestibular asymmetry. Gait Posture, 41, 820-4. doi: 10.1016/j.gaitpost.2015.02.019

LEE, A., BHATT, T. \& PAI, Y. C. 2016. Generalization of treadmill perturbation to overground slip during gait: Effect of different perturbation distances on slip recovery. J Biomech, 49, 14954. doi: 10.1016/j.jbiomech.2015.11.021

LEECH, K. A., ROEMMICH, R. T. \& BASTIAN, A. J. 2018. Creating flexible motor memories in human walking. Sci Rep, 8, 94. doi: 10.1038/s41598-017-18538-w

LEWIS, R. F. 2016. Vestibular implants studied in animal models: clinical and scientific implications. J Neurophysiol, 116, 2777-2788. doi: 10.1152/jn.00601.2016

LI, W., KEEGAN, T. H., STERNFELD, B., SIDNEY, S., QUESENBERRY, C. P., JR. \& KELSEY, J. L. 2006. Outdoor falls among middle-aged and older adults: a neglected public health problem. Am J Public Health, 96, 1192-200. doi: 10.2105/AJPH.2005.083055

LICHTWARK, G. A. \& WILSON, A. M. 2007. Is Achilles tendon compliance optimised for maximum muscle efficiency during locomotion? $J$ Biomech, 40, 1768-75. doi: 10.1016/j.jbiomech.2006.07.025

LIN, C. H., LIAO, K. C., PU, S. J., CHEN, Y. C. \& LIU, M. S. 2011. Associated factors for falls among the community-dwelling older people assessed by annual geriatric health examinations. PLoS One, 6, e18976. doi: 10.1371/journal.pone.0018976

LIPSITZ, L. A., MANOR, B., HABTEMARIAM, D., ILOPUTAIFE, I., ZHOU, J. \& TRAVISON, T. G. 2018. The pace and prognosis of peripheral sensory loss in advanced age: association with gait speed and falls. BMC Geriatr, 18, 274. doi: 10.1186/s12877-018-0970-5

LISTON, M. B., BAMIOU, D. E., MARTIN, F., HOPPER, A., KOOHI, N., LUXON, L. \& PAVLOU, M. 2014. Peripheral vestibular dysfunction is prevalent in older adults experiencing multiple non-syncopal falls versus age-matched non-fallers: a pilot study. Age Ageing, 43, 38-43. doi: 10.1093/ageing/aft129

LIU, X., BHATT, T. \& PAI, Y. C. 2016. Intensity and generalization of treadmill slip training: High or low, progressive increase or decrease? $J$ Biomech, 49, 135-40. doi: 10.1016/j.jbiomech.2015.06.004

LIU, X., BHATT, T., WANG, S., YANG, F. \& PAI, Y. C. 2017. Retention of the "first-trial effect" in gait-slip among community-living older adults. GeroScience, 39, 93-102. doi: 10.1007/s11357-017-9963-0

LONG, A. W., FINLEY, J. M. \& BASTIAN, A. J. 2015. A marching-walking hybrid induces step length adaptation and transfers to natural walking. J Neurophysiol, 113, 3905-14. doi: 10.1152/jn.00779.2014

LORD, S. R., DELBAERE, K. \& STURNIEKS, D. L. 2018. Aging. Handb Clin Neurol, 159, 157-171. doi: 10.1016/B978-0-444-63916-5.00010-0 
LORD, S. R., ROGERS, M. W., HOWLAND, A. \& FITZPATRICK, R. 1999. Lateral stability, sensorimotor function and falls in older people. J Am Geriatr Soc, 47, 1077-81.

LORD, S. R., SAMBROOK, P. N., GILBERT, C., KELLY, P. J., NGUYEN, T., WEBSTER, I. W. \& EISMAN, J. A. 1994a. Postural stability, falls and fractures in the elderly: results from the Dubbo Osteoporosis Epidemiology Study. Med J Aust, 160, 684-5, 688-91.

LORD, S. R., SHERRINGTON, C., MENZ, H. B. \& CLOSE, J. C. T. 2011. Falls in Older People: Risk Factors and Strategies for Prevention, New York, USA, Cambridge University Press.

LORD, S. R., WARD, J. A., WILLIAMS, P. \& ANSTEY, K. J. 1993. An epidemiological study of falls in older community-dwelling women: the Randwick falls and fractures study. Aust J Public Health, 17, 240-5.

LORD, S. R., WARD, J. A., WILLIAMS, P. \& ANSTEY, K. J. 1994b. Physiological factors associated with falls in older community-dwelling women. J Am Geriatr Soc, 42, 1110-7.

LUCHIES, C. W., WALlACE, D., PAZDUR, R., YOUNG, S. \& DEYOUNG, A. J. 1999. Effects of age on balance assessment using voluntary and involuntary step tasks. J Gerontol A Biol Sci Med Sci, 54, M140-4. doi: 10.1093/gerona/54.3.m140

LUCIEER, F., VONK, P., GUINAND, N., STOKROOS, R., KINGMA, H. \& VAN DE BERG, R. 2016. Bilateral Vestibular Hypofunction: Insights in Etiologies, Clinical Subtypes, and Diagnostics. Front Neurol, 7, 26. doi: 10.3389/fneur.2016.00026

LURIE, J. D., ZAGARIA, A. B., PIDGEON, D. M., FORMAN, J. L. \& SPRATT, K. F. 2013. Pilot comparative effectiveness study of surface perturbation treadmill training to prevent falls in older adults. BMC Geriatr, 13, 49. doi: 10.1186/1471-2318-13-49

LUTZ, W., SANDERSON, W. \& SCHERBOV, S. 2008. The coming acceleration of global population ageing. Nature, 451, 716-9. doi: 10.1038/nature06516

LUUKINEN, H., KOSKI, K., HILTUNEN, L. \& KIVELA, S. L. 1994. Incidence rate of falls in an aged population in northern Finland. J Clin Epidemiol, 47, 843-50.

MACKENZIE, L. \& MCINTYRE, A. 2019. How Do General Practitioners (GPs) Engage in Falls Prevention With Older People? A Pilot Survey of GPs in NHS England Suggests a Gap in Routine Practice to Address Falls Prevention. Front Public Health, 7, 32. doi: 10.3389/fpubh.2019.00032

MACKEY, D. C. \& ROBINOVITCH, S. N. 2005. Postural steadiness during quiet stance does not associate with ability to recover balance in older women. Clin Biomech, 20, 776-83. doi: 10.1016/j.clinbiomech.2005.05.002

MACKINNON, C. D. 2018. Sensorimotor anatomy of gait, balance, and falls. Handb Clin Neurol, 159, 3-26. doi: 10.1016/B978-0-444-63916-5.00001-X

MACNEILAGE, P. R. \& GLASAUER, S. 2017. Quantification of Head Movement Predictability and Implications for Suppression of Vestibular Input during Locomotion. Front Comput Neurosci, 11, 47. doi: 10.3389/fncom.2017.00047

MADEMLI, L. \& ARAMPATZIS, A. 2008. Mechanical and morphological properties of the triceps surae muscle-tendon unit in old and young adults and their interaction with a submaximal fatiguing contraction. J Electromyogr Kinesiol, 18, 89-98. doi: 10.1016/j.jelekin.2006.09.008

MADEMLI, L. \& ARAMPATZIS, A. 2014. Lower safety factor for old adults during walking at preferred velocity. Age, 36, 9636. doi: 10.1007/s11357-014-9636-1

MADIGAN, M. L. \& LLOYD, E. M. 2005. Age and stepping limb performance differences during a single-step recovery from a forward fall. J Gerontol A Biol Sci Med Sci, 60, 481-5. doi: $10.1093 /$ gerona/60.4.481 
MAGNUSSON, S. P., BEYER, N., ABRAHAMSEN, H., AAGAARD, P., NEERGAARD, K. \& KJAER, M. 2003. Increased cross-sectional area and reduced tensile stress of the Achilles tendon in elderly compared with young women. J Gerontol A Biol Sci Med Sci, 58, 123-7. doi: 10.1093/gerona/58.2.b123

MAHER, C. G., SHERRINGTON, C., HERBERT, R. D., MOSELEY, A. M. \& ELKINS, M. 2003. Reliability of the PEDro scale for rating quality of randomized controlled trials. Phys Ther, 83, 713-21.

MAKI, B. E., CHENG, K. C., MANSFIELD, A., SCOVIL, C. Y., PERRY, S. D., PETERS, A. L., MCKAY, S., LEE, T., MARQUIS, A., CORBEIL, P., FERNIE, G. R., LIU, B. \& MCILROY, W. E. 2008. Preventing falls in older adults: new interventions to promote more effective change-in-support balance reactions. $J$ Electromyogr Kinesiol, 18, 243-54. doi: 10.1016/j.jelekin.2007.06.005

MAKI, B. E., EDMONDSTONE, M. A. \& MCILROY, W. E. 2000. Age-related differences in laterally directed compensatory stepping behavior. J Gerontol A Biol Sci Med Sci, 55, M270-7. doi: 10.1093/gerona/55.5.m270

MAKI, B. E., HOLLIDAY, P. J. \& TOPPER, A. K. 1994. A prospective study of postural balance and risk of falling in an ambulatory and independent elderly population. $J$ Gerontol, 49, M72-84.

MAKI, B. E. \& MCILROY, W. E. 1996. Postural control in the older adult. Clin Geriatr Med, 12, 635-58.

MAKI, B. E. \& MCILROY, W. E. 1997. The role of limb movements in maintaining upright stance: the "change-in-support" strategy. Phys Ther, 77, 488-507. doi: 10.1093/ptj/77.5.488

MAKI, B. E. \& MCILROY, W. E. 2005. Change-in-support balance reactions in older persons: an emerging research area of clinical importance. Neurol Clin, 23, 751-83, vi-vii. doi: 10.1016/j.ncl.2005.01.002

MAKI, B. E. \& MCILROY, W. E. 2006. Control of rapid limb movements for balance recovery: agerelated changes and implications for fall prevention. Age Ageing, 35 Suppl 2, ii12-ii18. doi: 10.1093/ageing/af1078

MALMIVAARA, A., HELIOVAARA, M., KNEKT, P., REUNANEN, A. \& AROMAA, A. 1993. Risk factors for injurious falls leading to hospitalization or death in a cohort of 19,500 adults. Am J Epidemiol, 138, 384-94. doi: 10.1093/oxfordjournals.aje.a116871

MANCINI, C., WILliAMSON, D., BINKIN, N., MICHIElETTO, F., DE GIACOMI, G. V. \& GRUPPO DI LAVORO STUDIO, A. 2005. [Epidemiology of falls among the elderly]. Ig Sanita Pubbl, 61, 117-32.

MANsfield, A., AQUi, A., CENTEN, A., DANELls, C. J., DEPAUl, V. G., KNORR, S., SCHINKEL-IVY, A., BROOKS, D., INNESS, E. L., MCILROY, W. E. \& MOCHIZUKI, G. 2015a. Perturbation training to promote safe independent mobility post-stroke: study protocol for a randomized controlled trial. BMC Neurol, 15, 87. doi: 10.1186/s12883-0150347-8

MANSField, A., AQUI, A., DANELls, C. J., KNORR, S., CENTEN, A., DEPAUL, V. G., SCHINKEL-IVY, A., BROOKS, D., INNESS, E. L. \& MOCHIZUKI, G. 2018. Does perturbation-based balance training prevent falls among individuals with chronic stroke? A randomised controlled trial. BMJ Open, 8, e021510. doi: 10.1136/bmjopen-2018-021510 
MANSFIELD, A., INNESS, E. L., KOMAR, J., BIASIN, L., BRUNTON, K., LAKHANI, B. \& MCILROY, W. E. 2011. Training rapid stepping responses in an individual with stroke. Phys Ther, 91, 958-69. doi: 10.2522/ptj.20100212

MANSFIELD, A., PETERS, A. L., LIU, B. A. \& MAKI, B. E. 2007. A perturbation-based balance training program for older adults: study protocol for a randomised controlled trial. $B M C$ Geriatr, 7, 12. doi: 10.1186/1471-2318-7-12

MANSFIELD, A., PETERS, A. L., LIU, B. A. \& MAKI, B. E. 2010. Effect of a perturbation-based balance training program on compensatory stepping and grasping reactions in older adults: a randomized controlled trial. Phys Ther, 90, 476-91. doi: 10.2522/ptj.20090070

MANSFIELD, A., SCHINKEL-IVY, A., DANELLS, C. J., AQUI, A., ARYAN, R., BIASIN, L., POON, V., DEPAUL, V. \& INNESS, E. L. Does perturbation training prevent falls after discharge from stroke rehabilitation? A prospective cohort study with historical control. 2016 Canadian Stroke Congress, 2016 Québec City, QC, Canada.

MANSFIELD, A., WONG, J. S., BRYCE, J., KNORR, S. \& PATTERSON, K. K. 2015b. Does perturbation-based balance training prevent falls? Systematic review and meta-analysis of preliminary randomized controlled trials. Phys Ther, 95, 700-9. doi: 10.2522/ptj.20140090

MARCHAL-CRESPO, L., MICHELS, L., JAEGER, L., LOPEZ-OLORIZ, J. \& RIENER, R. 2017. Effect of Error Augmentation on Brain Activation and Motor Learning of a Complex Locomotor Task. Front Neurosci, 11, 526. doi: 10.3389/fnins.2017.00526

MARCHAL-CRESPO, L., SCHNEIDER, J., JAEGER, L. \& RIENER, R. 2014. Learning a locomotor task: with or without errors? J Neuroeng Rehabil, 11, 25. doi: 10.1186/1743-0003-11-25

MARIGOLD, D. S., ENG, J. J., DAWSON, A. S., INGLIS, J. T., HARRIS, J. E. \& GYLFADOTTIR, S. 2005. Exercise leads to faster postural reflexes, improved balance and mobility, and fewer falls in older persons with chronic stroke. J Am Geriatr Soc, 53, 416-23. doi: 10.1111/j.1532-5415.2005.53158.x

MARIGOLD, D. S. \& PATLA, A. E. 2002. Strategies for dynamic stability during locomotion on a slippery surface: effects of prior experience and knowledge. J Neurophysiol, 88, 339-53. doi: 10.1152/jn.00691.2001

MARTELLI, D., MONACO, V., BASSI LUCIANI, L. \& MICERA, S. 2013. Angular momentum during unexpected multidirectional perturbations delivered while walking. IEEE Trans Biomed Eng, 60, 1785-95. doi: 10.1109/TBME.2013.2241434

MARTELlI, D., VASHISTA, V., MICERA, S. \& AGRAWAL, S. K. 2016. Direction-Dependent Adaptation of Dynamic Gait Stability Following Waist-Pull Perturbations. IEEE Trans Neural Syst Rehabil Eng, 24, 1304-1313. doi: 10.1109/TNSRE.2015.2500100

MARTINEZ, M., VALENCIA, M., VIDORRETA, M., LUIS, E. O., CASTELLANOS, G., VILLAGRA, F., FERNANDEZ-SEARA, M. A. \& PASTOR, M. A. 2016. Trade-off between frequency and precision during stepping movements: Kinematic and BOLD brain activation patterns. Hum Brain Mapp, 37, 1722-37. doi: 10.1002/hbm.23131

MAZAHERI, M., HOOGKAMER, W., POTOCANAC, Z., VERSCHUEREN, S., ROERDINK, M., BEEK, P. J., PEPER, C. E. \& DUYSENS, J. 2015. Effects of aging and dual tasking on step adjustments to perturbations in visually cued walking. Exp Brain Res, 233, 3467-74. doi: 10.1007/s00221-015-4407-5

MCCRUM, C. 2018. Falls among older people-are intervention duration and specificity the keys to making a difference? BMJ, 361, k2213. doi: 10.1136/bmj.k2213 
MCCRUM, C., EPRO, G., MEIJER, K., ZIJLSTRA, W., BRÜGGEMANN, G. P. \& KARAMANIDIS, K. 2016a. Locomotor stability and adaptation during perturbed walking across the adult female lifespan. J Biomech, 49, 1244-1247. doi: 10.1016/j.jbiomech.2016.02.051

MCCRUM, C., ESSERS, J. M., JIE, L. J., LIU, W. Y. \& MEIJER, K. 2016b. Commentary: Older adults can improve compensatory stepping with repeated postural perturbations. Front Aging Neurosci, 8, 111. doi: 10.3389/fnagi.2016.00111

MCCRUM, C., EYSEL-GOSEPATH, K., EPRO, G., MEIJER, K., SAVElBERG, H. H., BRÜGGEMANN, G. P. \& KARAMANIDIS, K. 2014. Deficient recovery response and adaptive feedback potential in dynamic gait stability in unilateral peripheral vestibular disorder patients. Physiol Rep, 2, e12222. doi: 10.14814/phy2.12222

MCCRUM, C., EYSEL-GOSEPATH, K., EPRO, G., MEIJER, K., SAVELBERG, H. H., BRÜGGEMANN, G. P. \& KARAMANIDIS, K. 2017a. Associations Between Bipedal Stance Stability and Locomotor Stability Following a Trip in Unilateral Vestibulopathy. $J$ Appl Biomech, 33, 112-117. doi: 10.1123/jab.2016-0004

MCCRUM, C., GERARDS, M. H. G., KARAMANIDIS, K., ZIJLSTRA, W. \& MEIJER, K. 2017b. A systematic review of gait perturbation paradigms for improving reactive stepping responses and falls risk among healthy older adults. Eur Rev Aging Phys Act, 14, 3. doi: 10.1186/s11556-017-0173-7

MCCRUM, C., KARAMANIDIS, K., GREVENDONK, L., ZIJLSTRA, W. \& MEIJER, K. 2019. Older adults demonstrate interlimb transfer of reactive gait adaptations to repeated unpredictable gait perturbations. bioRxiv, 673574. doi: 10.1101/673574

MCCRUM, C., KARAMANIDIS, K., GREVENDONK, L., ZIJLSTRA, W. \& MEIJER, K. (Accepted 2019). Older adults demonstrate interlimb transfer of reactive gait adaptations to repeated unpredictable gait perturbations. GeroScience. doi: 10.1007/s11357-019-00130-x

MCCRUM, C., KARAMANIDIS, K., WILLEMS, P., ZIJLSTRA, W. \& MEIJER, K. 2018a. Retention, savings and interlimb transfer of reactive gait adaptations in humans following unexpected perturbations. Commun Biol, 1, 230. doi: 10.1038/s42003-018-0238-9

MCCRUM, C., LEOW, P., EPRO, G., KÖNIG, M., MEIJER, K. \& KARAMANIDIS, K. 2018b. Alterations in Leg Extensor Muscle-Tendon Unit Biomechanical Properties With Ageing and Mechanical Loading. Front Physiol, 9, 150. doi: 10.3389/fphys.2018.00150

MCCRUM, C., LUCIEER, F., VAN DE BERG, R., WILLEMS, P., PÉREZ FORNOS, A., GUINAND, N., KARAMANIDIS, K., KINGMA, H. \& MEIJER, K. 2018c. Is faster always better? The walking speed-dependency of gait variability in bilateral vestibulopathy. bioRxiv. doi: $10.1101 / 413955$

MCCRUM, C., WILLEMS, P., KARAMANIDIS, K. \& MEIJER, K. 2019a. Stability-normalised walking speed: A new approach for human gait perturbation research. J Biomech, 87, 4853. doi: 10.1016/j.jbiomech.2019.02.016

MCCRUM, C., WILLEMS, P., KARAMANIDIS, K. \& MEIJER, K. 2019b. Stability-normalised walking speed: a new approach for human gait perturbation research. bioRxiv. doi: $10.1101 / 314757$

MCCRUM, C., WILLEMS, P., VAN DE BERG, R., CAVUSCENS, S., GUINAND, N., GUYOT, J. P., PEREZ FORNOS, A., MARCELLIS, R., RANIERI, M., SENDEN, R., STOKROOS, R., ZIJLSTRA, W., KARAMANIDIS, K., MEIJER, K. \& KINGMA, H. 2016c. Preliminary observations of the acute effects of vestibular nerve stimulation on stride length and time 
in two patients with bilateral vestibular hypofunction. Gait \& Posture, 49, 124. doi: 10.1016/j.gaitpost.2016.07.179

MCGARVIE, L. A., MACDOUGALL, H. G., HALMAGYI, G. M., BURGESS, A. M., WEBER, K. P. \& CURTHOYS, I. S. 2015. The Video Head Impulse Test (vHIT) of Semicircular Canal Function - Age-Dependent Normative Values of VOR Gain in Healthy Subjects. Front Neurol, 6, 154. doi: 10.3389/fneur.2015.00154

MCILROY, W. E. \& MAKI, B. E. 1996. Age-related changes in compensatory stepping in response to unpredictable perturbations. J Gerontol A Biol Sci Med Sci, 51, M289-96. doi: 10.1093/gerona/51a.6.m289

MENANT, J. C., ST GEORGE, R. J., FITZPATRICK, R. C. \& LORD, S. R. 2012. Perception of the postural vertical and falls in older people. Gerontology, 58, 497-503. doi: 10.1159/000339295

MICKLE, K. J., MUNRO, B. J., LORD, S. R., MENZ, H. B. \& STEELE, J. R. 2009. ISB Clinical Biomechanics Award 2009: toe weakness and deformity increase the risk of falls in older people. Clin Biomech, 24, 787-91. doi: 10.1016/j.clinbiomech.2009.08.011

MIERAU, A., PESTER, B., HULSDUNKER, T., SCHIECKE, K., STRUDER, H. K. \& WITTE, H. 2017. Cortical Correlates of Human Balance Control. Brain Topogr, 30, 434-446. doi: 10.1007/s10548-017-0567-x

MILLE, M. L., JOHNSON-HILLIARD, M., MARTINEZ, K. M., ZHANG, Y., EDWARDS, B. J. \& ROGERS, M. W. 2013. One step, two steps, three steps more ... Directional vulnerability to falls in community-dwelling older people. J Gerontol A Biol Sci Med Sci, 68, 1540-8. doi: 10.1093/gerona/glt062

MILLE, M. L., JOHNSON, M. E., MARTINEZ, K. M. \& ROGERS, M. W. 2005. Age-dependent differences in lateral balance recovery through protective stepping. Clin Biomech, 20, 60716. doi: 10.1016/j.clinbiomech.2005.03.004

MOON, Y. \& SOSNOFF, J. J. 2017. Safe Landing Strategies During a Fall: Systematic Review and Meta-Analysis. Arch Phys Med Rehabil, 98, 783-794. doi: 10.1016/j.apmr.2016.08.460

MOREIRA, B. S., SAMPAIO, R. F. \& KIRKWOOD, R. N. 2015. Spatiotemporal gait parameters and recurrent falls in community-dwelling elderly women: a prospective study. Braz J Phys Ther, 19, 61-9. doi: 10.1590/bjpt-rbf.2014.0067

MORENO CATALA, M., WOITALLA, D. \& ARAMPATZIS, A. 2016. Reactive but not predictive locomotor adaptability is impaired in young Parkinson's disease patients. Gait Posture, 48, 177-182. doi: 10.1016/j.gaitpost.2016.05.008

MORTON, S. M. \& BASTIAN, A. J. 2006. Cerebellar contributions to locomotor adaptations during splitbelt treadmill walking. $J$ Neurosci, 26, 9107-16. doi: 10.1523/JNEUROSCI.262206.2006

MRACHACZ-KERSTING, N., GERVASIO, S. \& MARCHAND-PAUVERT, V. 2018. Evidence for a Supraspinal Contribution to the Human Crossed Reflex Response During Human Walking. Front Hum Neurosci, 12, 260. doi: 10.3389/fnhum.2018.00260

MUIR, S. W., BERG, K., CHESWORTH, B. \& SPEECHLEY, M. 2008. Use of the Berg Balance Scale for predicting multiple falls in community-dwelling elderly people: a prospective study. Phys Ther, 88, 449-59. doi: 10.2522/ptj.20070251

MÜLLER, R., HAUFLE, D. F. \& BLICKHAN, R. 2015. Preparing the leg for ground contact in running: the contribution of feed-forward and visual feedback. $J$ Exp Biol, 218, 451-7. doi: 10.1242/jeb.113688 
NASHNER, L. M. 1980. Balance adjustments of humans perturbed while walking. J Neurophysiol, 44, 650-64. doi: 10.1152/jn.1980.44.4.650

NIELSEN, J. B. \& SINKJAER, T. 2002. Afferent feedback in the control of human gait. $J$ Electromyogr Kinesiol, 12, 213-7.

NIINO, N., TSUZUKU, S., ANDO, F. \& SHIMOKATA, H. 2000. Frequencies and circumstances of falls in the National Institute for Longevity Sciences, Longitudinal Study of Aging (NILSLSA). J Epidemiol, 10, S90-4. doi: 10.2188/jea.10.1sup_90

NUTT, J. G., MARSDEN, C. D. \& THOMPSON, P. D. 1993. Human walking and higher-level gait disorders, particularly in the elderly. Neurology, 43, 268-79. doi: 10.1212/wnl.43.2.268

O'CONNOR, S. M., XU, H. Z. \& KUO, A. D. 2012. Energetic cost of walking with increased step variability. Gait Posture, 36, 102-7. doi: 10.1016/j.gaitpost.2012.01.014

O'LOUGHLIN, J. L., ROBITAILLE, Y., BOIVIN, J. F. \& SUISSA, S. 1993. Incidence of and risk factors for falls and injurious falls among the community-dwelling elderly. Am J Epidemiol, 137, 342-54. doi: 10.1093/oxfordjournals.aje.a116681

OATES, A., ARNOLD, C., WALKER-JOHNSTON, J., VAN OOTEGHEM, K., OLIVER, A., YAUSIE, J., LOUCKS, N., BAILEY, K., LEMIEUX, J. \& SIBLEY, K. M. 2017. Balance Assessment Practices of Saskatchewan Physiotherapists: A Brief Report of Survey Findings. Physiother Can, 69, 217-225. doi: 10.3138/ptc.2016-47

ODDSSON, L. I. E., BOISSY, P. \& MELZER, I. 2007. How to improve gait and balance function in elderly individuals-compliance with principles of training. Eur Rev Aging Phys Act, 4, 1523. doi: 10.1007/s11556-007-0019-9

OKUBO, Y., BRODIE, M. A., STURNIEKS, D. L., HICKS, C., CARTER, H., TOSON, B. \& LORD, S. R. 2018. Exposure to trips and slips with increasing unpredictability while walking can improve balance recovery responses with minimum predictive gait alterations. PLoS One, 13, e0202913. doi: 10.1371/journal.pone.0202913

OKUBO, Y., SCHOENE, D. \& LORD, S. R. 2017. Step training improves reaction time, gait and balance and reduces falls in older people: a systematic review and meta-analysis. $\mathrm{Br} J$ Sports Med, 51, 586-593. doi: 10.1136/bjsports-2015-095452

OLIJ, B. F., PANNEMAN, M. J. M., VAN BEECK, E. F., HAAGSMA, J. A., HARTHOLT, K. A. \& POLINDER, S. 2019. Fall-related healthcare use and mortality among older adults in the Netherlands, 1997-2016. Exp Gerontol, 120, 95-100. doi: 10.1016/j.exger.2019.03.003

OLUDARE, S. O., PATER, M. L., ROSENBLATT, N. J. \& GRABINER, M. D. 2018. Trip-specific training enhances recovery after large postural disturbances for which there is NO expectation. Gait Posture, 61, 382-386. doi: 10.1016/j.gaitpost.2018.02.001

ORCES, C. H. 2013. Prevalence and Determinants of Falls among Older Adults in Ecuador: An Analysis of the SABE I Survey. Curr Gerontol Geriatr Res, 2013, 495468. doi: $10.1155 / 2013 / 495468$

ORCES, C. H. \& ALAMGIR, H. 2014. Trends in fall-related injuries among older adults treated in emergency departments in the USA. Inj Prev, 20, 421-3. doi: 10.1136/injuryprev-2014041268

ORTMAN, J. M., VELKOFF, V. A. \& HOGAN, H. 2014. An Aging Nation: The Older Population in the United States. In: BUREAU, U. S. C. (ed.). U.S. Census Bureau.

OVERSTALL, P. W., EXTON-SMITH, A. N., IMMS, F. J. \& JOHNSON, A. L. 1977. Falls in the elderly related to postural imbalance. $\mathrm{Br}$ Med J, 1, 261-4. doi: 10.1136/bmj.1.6056.261 
OWINGS, T. M. \& GRABINER, M. D. 2003. Measuring step kinematic variability on an instrumented treadmill: how many steps are enough? J Biomech, 36, 1215-8. doi: 10.1016/s0021-9290(03)00108-8

OWINGS, T. M., PAVOL, M. J., FOLEY, K. T. \& GRABINER, M. D. 2000. Measures of postural stability are not predictors of recovery from large postural disturbances in healthy older adults. J Am Geriatr Soc, 48, 42-50.

PAI, Y. C., BHATT, T., WANG, E., ESPY, D. \& PAVOL, M. J. 2010. Inoculation against falls: rapid adaptation by young and older adults to slips during daily activities. Arch Phys Med Rehabil, 91, 452-9. doi: 10.1016/j.apmr.2009.10.032

PAI, Y. C., BHATT, T., YANG, F. \& WANG, E. 2014a. Perturbation training can reduce communitydwelling older adults' annual fall risk: a randomized controlled trial. J Gerontol A Biol Sci Med Sci, 69, 1586-94. doi: 10.1093/gerona/glu087

PAI, Y. C. \& BHATT, T. S. 2007. Repeated-slip training: an emerging paradigm for prevention of slip-related falls among older adults. Phys Ther, 87, 1478-91. doi: 10.2522/ptj.20060326

PAI, Y. C. \& PATTON, J. 1997. Center of mass velocity-position predictions for balance control. $J$ Biomech, 30, 347-54.

PAI, Y. C., YANG, F., BHATT, T. \& WANG, E. 2014b. Learning from laboratory-induced falling: long-term motor retention among older adults. Age, 36, 9640. doi: 10.1007/s11357-014-96405

PALUMBO, P., KLENK, J., CATTELANI, L., BANDINELlI, S., FERRUCCI, L., RAPP, K., CHIARI, L. \& ROTHENBACHER, D. 2016. Predictive Performance of a Fall Risk Assessment Tool for Community-Dwelling Older People (FRAT-up) in 4 European Cohorts. J Am Med Dir Assoc, 17, 1106-1113. doi: 10.1016/j.jamda.2016.07.015

PANDY, M. G. \& ANDRIACCHI, T. P. 2010. Muscle and joint function in human locomotion. Annu Rev Biomed Eng, 12, 401-33. doi: 10.1146/annurev-bioeng-070909-105259

PANG, M. Y., LAM, T. \& YANG, J. F. 2003. Infants adapt their stepping to repeated trip-inducing stimuli. J Neurophysiol, 90, 2731-40. doi: 10.1152/jn.00407.2003

PARASKEVOUDI, N., BALCI, F. \& VATAKIS, A. 2018. "Walking" through the sensory, cognitive, and temporal degradations of healthy aging. Ann N Y Acad Sci. doi: 10.1111/nyas.13734

PARIJAT, P. \& LOCKHART, T. E. 2012. Effects of moveable platform training in preventing slipinduced falls in older adults. Ann Biomed Eng, 40, 1111-21. doi: 10.1007/s10439-011-04770

PARKKARI, J., KANNUS, P., PALVANEN, M., NATRI, A., VAINIO, J., AHO, H., VUORI, I. \& JARVINEN, M. 1999. Majority of hip fractures occur as a result of a fall and impact on the greater trochanter of the femur: a prospective controlled hip fracture study with 206 consecutive patients. Calcif Tissue Int, 65, 183-7.

PATEL, P. \& BHATT, T. 2015. Adaptation to large-magnitude treadmill-based perturbations: improvements in reactive balance response. Physiol Rep, 3. doi: 10.14814/phy2.12247

PATER, M. L., ROSENBLATT, N. J. \& GRABINER, M. D. 2015. Expectation of an upcoming large postural perturbation influences the recovery stepping response and outcome. Gait Posture, 41, 335-7. doi: 10.1016/j.gaitpost.2014.10.026

PATLA, A., FRANK, J. \& WINTER, D. 1990. Assessment of balance control in the elderly: major issues. Physiother Can, 42, 89-97. doi: 10.3138/ptc.42.2.089 
PATLA, A. E. 1991. Understanding the Control of Human Locomotion: A Prologue. In: PATLA, A. E. (ed.) Adaptability of Human Gait - Implications for the Control of Locomotion. NorthHolland. doi: 10.1016/s0166-4115(08)60735-9

PAVOL, M. J., OWINGS, T. M., FOLEY, K. T. \& GRABINER, M. D. 1999. Gait characteristics as risk factors for falling from trips induced in older adults. J Gerontol A Biol Sci Med Sci, 54, M583-90. doi: 10.1093/gerona/54.11.m583

PAVOL, M. J., OWINGS, T. M., FOLEY, K. T. \& GRABINER, M. D. 2002a. Influence of lower extremity strength of healthy older adults on the outcome of an induced trip. J Am Geriatr Soc, 50, 256-62.

PAVOL, M. J., RUNTZ, E. F., EDWARDS, B. J. \& PAI, Y. C. 2002b. Age influences the outcome of a slipping perturbation during initial but not repeated exposures. J Gerontol A Biol Sci Med Sci, 57, M496-503. doi: 10.1093/gerona/57.8.m496

PAVOL, M. J., RUNTZ, E. F. \& PAI, Y. C. 2004. Young and older adults exhibit proactive and reactive adaptations to repeated slip exposure. J Gerontol A Biol Sci Med Sci, 59, 494-502. doi: 10.1093/gerona/59.5.m494

PEREZ FORNOS, A., CAVUSCENS, S., RANIERI, M., VAN DE BERG, R., STOKROOS, R., KINGMA, H., GUYOT, J. P. \& GUINAND, N. 2017. The vestibular implant: A probe in orbit around the human balance system. J Vestib Res, 27, 51-61. doi: 10.3233/VES-170604

PEREZ FORNOS, A., GUINAND, N., VAN DE BERG, R., STOKROOS, R., MICERA, S., KINGMA, H., PELIZZONE, M. \& GUYOT, J. P. 2014. Artificial balance: restoration of the vestibuloocular reflex in humans with a prototype vestibular neuroprosthesis. Front Neurol, 5, 66. doi: 10.3389/fneur.2014.00066

PERRY, J. A. \& SRINIVASAN, M. 2017. Walking with wider steps changes foot placement control, increases kinematic variability and does not improve linear stability. $R$ Soc Open Sci, 4, 160627. doi: 10.1098/rsos.160627

PETERSON, D. S., DIJKSTRA, B. W. \& HORAK, F. B. 2016. Postural motor learning in people with Parkinson's disease. J Neurol, 263, 1518-29. doi: 10.1007/s00415-016-8158-4

PETERSON, D. S. \& HORAK, F. B. 2016. Neural Control of Walking in People with Parkinsonism. Physiology, 31, 95-107. doi: 10.1152/physiol.00034.2015

PIJNAPPELS, M., BOBBERT, M. F. \& VAN DIEËN, J. H. 2005a. Control of support limb muscles in recovery after tripping in young and older subjects. Exp Brain Res, 160, 326-33. doi: 10.1007/s00221-004-2014-y

PIJNAPPELS, M., BOBBERT, M. F. \& VAN DIEËN, J. H. 2005b. How early reactions in the support limb contribute to balance recovery after tripping. J Biomech, 38, 627-34. doi: 10.1016/j.jbiomech.2004.03.029

PIJNAPPELS, M., BOBBERT, M. F. \& VAN DIEËN, J. H. 2005c. Push-off reactions in recovery after tripping discriminate young subjects, older non-fallers and older fallers. Gait Posture, 21, 388-94. doi: 10.1016/j.gaitpost.2004.04.009

PIJNAPPELS, M., REEVES, N. D., MAGANARIS, C. N. \& VAN DIEËN, J. H. 2008a. Tripping without falling; lower limb strength, a limitation for balance recovery and a target for training in the elderly. $J$ Electromyogr Kinesiol, 18, 188-96. doi: 10.1016/j.jelekin.2007.06.004

PIJNAPPELS, M., VAN DER BURG, P. J., REEVES, N. D. \& VAN DIEËN, J. H. 2008b. Identification of elderly fallers by muscle strength measures. Eur J Appl Physiol, 102, 58592. doi: 10.1007/s00421-007-0613-6 
PIKER, E. G., JACOBSON, G. P., BURKARD, R. F., MCCASLIN, D. L. \& HOOD, L. J. 2013. Effects of age on the tuning of the cVEMP and oVEMP. Ear Hear, 34, e65-73. doi: 10.1097/AUD.0b013e31828fc9f2

POLLOCK, A. S., DURWARD, B. R., ROWE, P. J. \& PAUL, J. P. 2000. What is balance? Clin Rehabil, 14, 402-6. doi: 10.1191/0269215500cr342oa

POTOCANAC, Z., DE BRUIN, J., VAN DER VEEN, S., VERSCHUEREN, S., VAN DIEËN, J., DUYSENS, J. \& PIJNAPPELS, M. 2014. Fast online corrections of tripping responses. Exp Brain Res, 232, 3579-90. doi: 10.1007/s00221-014-4038-2

POTOCANAC, Z., SMULDERS, E., PIJNAPPELS, M., VERSCHUEREN, S. \& DUYSENS, J. 2015. Response inhibition and avoidance of virtual obstacles during gait in healthy young and older adults. Hum Mov Sci, 39, 27-40. doi: 10.1016/j.humov.2014.08.015

PROKOP, T., BERGER, W., ZIJLSTRA, W. \& DIETZ, V. 1995. Adaptational and learning processes during human split-belt locomotion: interaction between central mechanisms and afferent input. Exp Brain Res, 106, 449-56.

PROTAS, E. J., MITCHELL, K., WILLIAMS, A., QURESHY, H., CAROLINE, K. \& LAI, E. C. 2005. Gait and step training to reduce falls in Parkinson's disease. NeuroRehabilitation, 20, 18390.

PRUDHAM, D. \& EVANS, J. G. 1981. Factors associated with falls in the elderly: a community study. Age Ageing, 10, 141-6. doi: 10.1093/ageing/10.3.141

QUINTERN, J., BERGER, W. \& DIETZ, V. 1985. Compensatory reactions to gait perturbations in man: short- and long-term effects of neuronal adaptation. Neurosci Lett, 62, 371-6.

RAPP, K., FREIBERGER, E., TODD, C., KLENK, J., BECKER, C., DENKINGER, M., SCHEIDTNAVE, C. \& FUCHS, J. 2014. Fall incidence in Germany: results of two population-based studies, and comparison of retrospective and prospective falls data collection methods. BMC Geriatr, 14, 105. doi: 10.1186/1471-2318-14-105

RECHEL, B., GRUNDY, E., ROBINE, J. M., CYLUS, J., MACKENBACH, J. P., KNAI, C. \& MCKEE, M. 2013. Ageing in the European Union. Lancet, 381, 1312-22. doi: 10.1016/S01406736(12)62087-X

REISMAN, D. S., WITYK, R., SILVER, K. \& BASTIAN, A. J. 2009. Split-belt treadmill adaptation transfers to overground walking in persons poststroke. Neurorehabil Neural Repair, 23, 735-44. doi: 10.1177/1545968309332880

REYES-ORTIZ, C. A., AL SNIH, S. \& MARKIDES, K. S. 2005. Falls among elderly persons in Latin America and the Caribbean and among elderly Mexican-Americans. Rev Panam Salud Publica, 17, 362-9. doi: 10.1590/s1020-49892005000500008

RICHARDSON, J. K. \& HURVITZ, E. A. 1995. Peripheral neuropathy: a true risk factor for falls. J Gerontol A Biol Sci Med Sci, 50, M211-5. doi: 10.1093/gerona/50a.4.m211

RINGHOF, S. \& STEIN, T. 2018. Biomechanical assessment of dynamic balance: Specificity of different balance tests. Hum Mov Sci, 58, 140-147. doi: 10.1016/j.humov.2018.02.004

RIVA, F., BISI, M. C. \& STAGNI, R. 2014. Gait variability and stability measures: minimum number of strides and within-session reliability. Comput Biol Med, 50, 9-13. doi: 10.1016/j.compbiomed.2014.04.001

ROBBINS, S., WAKED, E. \& MCCLARAN, J. 1995. Proprioception and stability: foot position awareness as a function of age and footwear. Age Ageing, 24, 67-72. doi: 10.1093/ageing/24.1.67 
ROBERTS, T. J. 2002. The integrated function of muscles and tendons during locomotion. Comp Biochem Physiol A Mol Integr Physiol, 133, 1087-99.

ROBINOVITCH, S. 2018. Ecology of falls. Handb Clin Neurol, 159, 147-154. doi: 10.1016/B978-0444-63916-5.00009-4

ROBINOVITCH, S. N., FELDMAN, F., YANG, Y., SCHONNOP, R., LEUNG, P. M., SARRAF, T., SIMS-GOULD, J. \& LOUGHIN, M. 2013. Video capture of the circumstances of falls in elderly people residing in long-term care: an observational study. Lancet, 381, 47-54. doi: 10.1016/S0140-6736(12)61263-X

ROBINOVITCH, S. N., INKSTER, L., MAURER, J. \& WARNICK, B. 2003. Strategies for avoiding hip impact during sideways falls. $J$ Bone Miner Res, 18, 1267-73. doi: 10.1359/jbmr.2003.18.7.1267

ROEMMICH, R. T. \& BASTIAN, A. J. 2015. Two ways to save a newly learned motor pattern. $J$ Neurophysiol, 113, 3519-30. doi: 10.1152/jn.00965.2014

ROGERS, M. W., HEDMAN, L. D., JOHNSON, M. E., CAIN, T. D. \& HANKE, T. A. 2001. Lateral stability during forward-induced stepping for dynamic balance recovery in young and older adults. J Gerontol A Biol Sci Med Sci, 56, M589-94. doi: 10.1093/gerona/56.9.m589

ROSEnBLATT, N. J., MARONE, J. \& GRABINER, M. D. 2013. Preventing trip-related falls by community-dwelling adults: a prospective study. J Am Geriatr Soc, 61, 1629-31. doi: 10.1111 jgs. 12428

ROUDSARI, B. S., EBEL, B. E., CORSO, P. S., MOLINARI, N. A. \& KOEPSELL, T. D. 2005. The acute medical care costs of fall-related injuries among the U.S. older adults. Injury, 36, 1316-22. doi: 10.1016/j.injury.2005.05.024

SAI, A. J., GALLAGHER, J. C., SMITH, L. M. \& LOGSDON, S. 2010. Fall predictors in the community dwelling elderly: a cross sectional and prospective cohort study. J Musculoskelet Neuronal Interact, 10, 142-50.

SAKAI, M., SHIBA, Y., SATO, H. \& TAKAHIRA, N. 2008. Motor Adaptation during Slip-Perturbed Gait in Older Adults. J Phys Ther Sci, 20, 109-115. doi: 10.1589/jpts.20.109

SANTUZ, A., EKIZOS, A., ECKARDT, N., KIBELE, A. \& ARAMPATZIS, A. 2018. Challenging human locomotion: stability and modular organisation in unsteady conditions. Sci Rep, 8, 2740. doi: 10.1038/s41598-018-21018-4

SATtin, R. W., LAMBERT HUBER, D. A., DEVito, C. A., RODRIGUEZ, J. G., ROS, A., BACCHELLI, S., STEVENS, J. A. \& WAXWEILER, R. J. 1990. The incidence of fall injury events among the elderly in a defined population. Am J Epidemiol, 131, 1028-37. doi: 10.1093/oxfordjournals.aje.a115594

SAYER, A. A., SYDDALL, H. E., MARTIN, H. J., DENNISON, E. M., ANDERSON, F. H. \& COOPER, C. 2006. Falls, sarcopenia, and growth in early life: findings from the Hertfordshire cohort study. Am J Epidemiol, 164, 665-71. doi: 10.1093/aje/kwj255

SCHLICK, C., SCHNIEPP, R., LOIDL, V., WUEHR, M., HESSELBARTH, K. \& JAHN, K. 2016. Falls and fear of falling in vertigo and balance disorders: A controlled cross-sectional study. $J$ Vestib Res, 25, 241-51. doi: 10.3233/VES-150564

SCHNEIDER, C. \& CAPADAY, C. 2003. Progressive adaptation of the soleus H-reflex with daily training at walking backward. J Neurophysiol, 89, 648-56. doi: 10.1152/jn.00403.2002

SCHNIEPP, R., SCHLICK, C., SCHENKEL, F., PRADHAN, C., JAHN, K., BRANDT, T. \& WUEHR, M. 2017. Clinical and neurophysiological risk factors for falls in patients with bilateral vestibulopathy. J Neurol, 264, 277-283. doi: 10.1007/s00415-016-8342-6 
SCHNIEPP, R., WUEHR, M., NEUHAEUSSER, M., KAMENOVA, M., DIMITRIADIS, K., KLOPSTOCK, T., STRUPP, M., BRANDT, T. \& JAHN, K. 2012. Locomotion speed determines gait variability in cerebellar ataxia and vestibular failure. Mov Disord, 27, 12531. doi: $10.1002 / \mathrm{mds} .23978$

SCHRAGER, M. A., KELLY, V. E., PRICE, R., FERRUCCI, L. \& SHUMWAY-COOK, A. 2008. The effects of age on medio-lateral stability during normal and narrow base walking. Gait Posture, 28, 466-71. doi: 10.1016/j.gaitpost.2008.02.009

SCHULTZ, A. B. 1992. Mobility impairment in the elderly: challenges for biomechanics research. J Biomech, 25, 519-28.

SCHUMACHER, J., PIENTKA, L., TRAMPISCH, U., MOSCHNY, A., HINRICHS, T. \& THIEM, U. 2014. The prevalence of falls in adults aged 40 years or older in an urban, German population. Results from a telephone survey. $Z$ Gerontol Geriatr, 47, 141-6. doi: 10.1007/s00391-013-0503-y

SELINGER, J. C., O'CONNOR, S. M., WONG, J. D. \& DONELAN, J. M. 2015. Humans Can Continuously Optimize Energetic Cost during Walking. Curr Biol, 25, 2452-6. doi: 10.1016/j.cub.2015.08.016

SENDEN, R., SAVELBERG, H. H., ADAM, J., GRIMM, B., HEYLIGERS, I. C. \& MEIJER, K. 2014. The influence of age, muscle strength and speed of information processing on recovery responses to external perturbations in gait. Gait Posture, 39, 513-7. doi: 10.1016/j.gaitpost.2013.08.033

SHANKAR, K. N., TREADWAY, N. J., TAYLOR, A. A., BREAUD, A. H., PETERSON, E. W. \& HOWLAND, J. 2017. Older adult falls prevention behaviors 60 days post-discharge from an urban emergency department after treatment for a fall. Inj Epidemiol, 4, 18. doi: 10.1186/s40621-017-0114-y

SHAPIRO, A. \& MELZER, I. 2010. Balance perturbation system to improve balance compensatory responses during walking in old persons. J Neuroeng Rehabil, 7, 32. doi: 10.1186/1743-00037-32

SHARMA, P. K., BUNKER, C. H., SINGH, T., GANGULY, E., REDDY, P. S., NEWMAN, A. B. \& CAULEY, J. A. 2017. Burden and Correlates of Falls among Rural Elders of South India: Mobility and Independent Living in Elders Study. Curr Gerontol Geriatr Res, 2017, 1290936. doi: 10.1155/2017/1290936

SHELDON, J. H. 1960. On the Natural History of Falls in Old Age. Br Med J, 2, 1685-90. doi: 10.1136/bmj.2.5214.1685

SHEN, X. \& MAK, M. K. 2015. Technology-assisted balance and gait training reduces falls in patients with Parkinson's disease: a randomized controlled trial with 12-month follow-up. Neurorehabil Neural Repair, 29, 103-11. doi: 10.1177/1545968314537559

SHERRINGTON, C., FAIRHALL, N. J., WALLBANK, G. K., TIEDEMANN, A., MICHALEFF, Z. A., HOWARD, K., CLEMSON, L., HOPEWELL, S. \& LAMB, S. E. 2019. Exercise for preventing falls in older people living in the community. Cochrane Database Syst Rev, 1, CD012424. doi: 10.1002/14651858.CD012424.pub2

SHERRINGTON, C., MICHALEFF, Z. A., FAIRHALL, N., PAUL, S. S., TIEDEMANN, A., WHITNEY, J., CUMMinG, R. G., HERBERT, R. D., CLOSE, J. C. T. \& LORD, S. R. 2017. Exercise to prevent falls in older adults: an updated systematic review and meta-analysis. Br J Sports Med, 51, 1750-1758. doi: 10.1136/bjsports-2016-096547 
SHERRINGTON, C., WHITNEy, J. C., LORD, S. R., HERBERT, R. D., CUMMING, R. G. \& CLOSE, J. C. 2008. Effective exercise for the prevention of falls: a systematic review and meta-analysis. J Am Geriatr Soc, 56, 2234-43. doi: 10.1111/j.1532-5415.2008.02014.x

SHIMADA, H., OBUCHI, S., FURUNA, T. \& SUZUKI, T. 2004. New Intervention Program for Preventing Falls Among Frail Elderly People. Am J Phys Med Rehabil, 83, 493-499. doi: 10.1097/01.Phm.0000130025.54168.91

SIBLEY, K. M., BENTLEY, D. C., SALBACH, N. M., GARDNER, P., MCGLYNN, M., O'HOSKI, S., SHAFFER, J., SHING, P., MCEWEN, S., BEAUCHAMP, M. K., HOSSAIN, S., STRAUS, S. E. \& JAGLAL, S. B. 2018. A theory-based multi-component intervention to increase reactive balance measurement by physiotherapists in three rehabilitation hospitals: an uncontrolled single group study. BMC Health Serv Res, 18, 724. doi: 10.1186/s12913-018-3533-8

SIBLEY, K. M., INNESS, E. L., STRAUS, S. E., SALBACH, N. M. \& JAGLAL, S. B. 2013. Clinical assessment of reactive postural control among physiotherapists in Ontario, Canada. Gait Posture, 38, 1026-31. doi: 10.1016/j.gaitpost.2013.05.016

SIBLEY, K. M., STRAUS, S. E., INNESS, E. L., SALBACH, N. M. \& JAGLAL, S. B. 2011. Balance assessment practices and use of standardized balance measures among Ontario physical therapists. Phys Ther, 91, 1583-91. doi: 10.2522/ptj.20110063

SINGER, J. C., PRENTICE, S. D. \& MCILROY, W. E. 2016. Age-related challenges in reactive control of mediolateral stability during compensatory stepping: A focus on the dynamics of restabilisation. J Biomech, 49, 749-755. doi: 10.1016/j.jbiomech.2016.02.001

SIQUEIRA, F. V., FACCHINI, L. A., SILVEIRA, D. S., PICCINI, R. X., TOMASI, E., THUME, E., SILVA, S. M. \& DILELIO, A. 2011. Prevalence of falls in elderly in Brazil: a countrywide analysis. Cad Saude Publica, 27, 1819-26.

SKELTON, D. A., KENNEDY, J. \& RUTHERFORD, O. M. 2002. Explosive power and asymmetry in leg muscle function in frequent fallers and non-fallers aged over 65. Age Ageing, 31, 11925. doi: 10.1093/ageing/31.2.119

SLOAN, L. L. 1959. New test charts for the measurement of visual acuity at far and near distances. Am J Ophthalmol, 48, 807-13.

SLOOT, L. H., VAN DEN NOORT, J. C., VAN DER KROGT, M. M., BRUIJN, S. M. \& HARLAAR, J. 2015. Can Treadmill Perturbations Evoke Stretch Reflexes in the Calf Muscles? PLoS One, 10, e0144815. doi: 10.1371/journal.pone.0144815

SLOOT, L. H., VAN DER KROGT, M. M. \& HARLAAR, J. 2014. Effects of adding a virtual reality environment to different modes of treadmill walking. Gait Posture, 39, 939-45. doi: 10.1016/j.gaitpost.2013.12.005

SMANIA, N., CORATO, E., TINAZZI, M., STANZANI, C., FIASCHI, A., GIRARDI, P. \& GANDOLFI, M. 2010. Effect of balance training on postural instability in patients with idiopathic Parkinson's disease. Neurorehabil Neural Repair, 24, 826-34. doi: 10.1177/1545968310376057

SMEESTERS, C., HAYES, W. C. \& MCMAHON, T. A. 2001. Disturbance type and gait speed affect fall direction and impact location. J Biomech, 34, 309-17.

SOUSA, A. S., SILVA, A. \& TAVARES, J. M. 2012. Biomechanical and neurophysiological mechanisms related to postural control and efficiency of movement: a review. Somatosens Mot Res, 29, 131-43. doi: 10.3109/08990220.2012.725680 
SPAICH, E. G., ARENDT-NIELSEN, L. \& ANDERSEN, O. K. 2004. Modulation of lower limb withdrawal reflexes during gait: a topographical study. $J$ Neurophysiol, 91, 258-66. doi: $10.1152 /$ jn.00360.2003

SPRENGER, A., WOJAK, J. F., JANDL, N. M. \& HELMCHEN, C. 2017. Postural Control in Bilateral Vestibular Failure: Its Relation to Visual, Proprioceptive, Vestibular, and Cognitive Input. Front Neurol, 8, 444. doi: 10.3389/fneur.2017.00444

STEnRoth, L., CRONIN, N. J., PELTONEN, J., KORHONEN, M. T., SIPILA, S. \& FINNI, T. 2016. Triceps surae muscle-tendon properties in older endurance- and sprint-trained athletes. J Appl Physiol, 120, 63-9. doi: 10.1152/japplphysiol.00511.2015

STEnRoTh, L., PELTONEN, J., CRONIN, N. J., SIPILA, S. \& FINNI, T. 2012. Age-related differences in Achilles tendon properties and triceps surae muscle architecture in vivo. $J$ Appl Physiol, 113, 1537-44. doi: 10.1152/japplphysiol.00782.2012

STERGIOU, N. \& DECKER, L. M. 2011. Human movement variability, nonlinear dynamics, and pathology: is there a connection? Hum Mov Sci, 30, 869-888.

STEVENS, J. A., CORSO, P. S., FINKELSTEIN, E. A. \& MILLER, T. R. 2006. The costs of fatal and non-fatal falls among older adults. Inj Prev, 12, 290-5. doi: 10.1136/ip.2005.011015

STEvenson, A. J., GEertsen, S. S., ANDERSEN, J. B., SINKJAER, T., NIELSEN, J. B. \& MRACHACZ-KERSTING, N. 2013. Interlimb communication to the knee flexors during walking in humans. J Physiol, 591, 4921-35. doi: 10.1113/jphysiol.2013.257949

STEVEnson, A. J., GeERTSEN, S. S., SINKJAER, T., NIELSEN, J. B. \& MRACHACZKERSTING, N. 2015. Interlimb communication following unexpected changes in treadmill velocity during human walking. $J$ Neurophysiol, 113, 3151-8. doi: 10.1152/jn.00794.2014

STEWART WILLIAMS, J., KOWAL, P., HESTEKIN, H., O'DRISCOLL, T., PELTZER, K., YAWSON, A., BIRITWUM, R., MAXIMOVA, T., SALINAS RODRIGUEZ, A., MANRIQUE ESPINOZA, B., WU, F., AROKIASAMY, P., CHATTERJI, S. \& SAGE COLLABORATORS. 2015. Prevalence, risk factors and disability associated with fall-related injury in older adults in low- and middle-incomecountries: results from the WHO Study on global AGEing and adult health (SAGE). BMC Med, 13, 147. doi: 10.1186/s12916-015-0390-8

STRUPP, M., KIM, J. S., MUROFUSHI, T., STRAUMANN, D., JEN, J. C., ROSENGREN, S. M., DELLA SANTINA, C. C. \& KINGMA, H. 2017. Bilateral vestibulopathy: Diagnostic criteria Consensus document of the Classification Committee of the Barany Society. J Vestib Res, 27, 177-189. doi: 10.3233/VES-170619

STUBBS, P. W., NIELSEN, J. F., SINKJAER, T. \& MRACHACZ-KERSTING, N. 2011. Phase modulation of the short-latency crossed spinal response in the human soleus muscle. $J$ Neurophysiol, 105, 503-11. doi: 10.1152/jn.00786.2010

STURNIEKS, D. L., MENANT, J., DELBAERE, K., VANRENTERGHEM, J., ROGERS, M. W., FITZPATRICK, R. C. \& LORD, S. R. 2013. Force-controlled balance perturbations associated with falls in older people: a prospective cohort study. PLoS One, 8, e70981. doi: 10.1371/journal.pone.0070981

SUN, R., CUI, C. \& SHEA, J. B. 2017. Aging effect on step adjustments and stability control in visually perturbed gait initiation. Gait Posture, 58, 268-273. doi: 10.1016/j.gaitpost.2017.08.013

SÜPTITZ, F., KARAMANIDIS, K., MORENO CATALA, M. \& BRÜGGEMANN, G. P. 2012. Symmetry and reproducibility of the components of dynamic stability in young adults at 
different walking velocities on the treadmill. J Electromyogr Kinesiol, 22, 301-7. doi: 10.1016/j.jelekin.2011.12.007

SÜPTITZ, F., MORENO CATALA, M., BRÜGGEMANN, G. P. \& KARAMANIDIS, K. 2013. Dynamic stability control during perturbed walking can be assessed by a reduced kinematic model across the adult female lifespan. Hum Mov Sci, 32, 1404-14. doi: 10.1016/j.humov.2013.07.008

SUTEERAWATTANANON, M., MACNEILL, B. \& PROTAS, E. J. 2002. Supported treadmill training for gait and balance in a patient with progressive supranuclear palsy. Phys Ther, 82, 485-95.

SWANENBURG, J., DE BRUIN, E. D., UEBELHART, D. \& MULDER, T. 2010. Falls prediction in elderly people: a 1-year prospective study. Gait Posture, 31, 317-21. doi: 10.1016/j.gaitpost.2009.11.013

TALBOT, L. A., MUSIOL, R. J., WITHAM, E. K. \& METTER, E. J. 2005. Falls in young, middleaged and older community dwelling adults: perceived cause, environmental factors and injury. BMC Public Health, 5, 86. doi: 10.1186/1471-2458-5-86

TANG, P.-F. \& WOOLLACOTT, M. H. 1996. Balance Control in Older Adults: Training Effects on Balance Control and the Integration of Balance Control into Walking. In: FERRANDEZ, A.-M. \& TEASDALE, N. (eds.) Changes in Sensory Motor Behavior in Aging. NorthHolland. doi: 10.1016/s0166-4115(96)80015-x

TEASELL, R., BAYONA, N., MARSHALL, S., CULLEN, N., BAYLEY, M., CHUNDAMALA, J., VILLAMERE, J., MACKIE, D., REES, L., HARTRIDGE, C., LIPPERT, C., HILDITCH, M., WELCH-WEST, P., WEISER, M., FERRI, C., MCCABE, P., MCCORMICK, A., AUBUT, J. A., COMPER, P., SALTER, K., VAN REEKUM, R., COLLINS, D., FOLEY, N., NOWAK, J., JUTAI, J., SPEECHLEY, M., HELLINGS, C. \& TU, L. 2007. A systematic review of the rehabilitation of moderate to severe acquired brain injuries. Brain Inj, 21, 107-12. doi: 10.1080/02699050701201524

TENO, J., KIEL, D. P. \& MOR, V. 1990. Multiple stumbles: a risk factor for falls in communitydwelling elderly. A prospective study. J Am Geriatr Soc, 38, 1321-5.

TERRIER, P. \& REYNARD, F. 2015. Effect of age on the variability and stability of gait: a crosssectional treadmill study in healthy individuals between 20 and 69 years of age. Gait Posture, 41, 170-4. doi: 10.1016/j.gaitpost.2014.09.024

TERROSO, M., ROSA, N., TORRES MARQUES, A. \& SIMOES, R. 2013. Physical consequences of falls in the elderly: a literature review from 1995 to 2010. Eur Rev Aging Phys Act, 11, 5159. doi: 10.1007/s11556-013-0134-8

THELEN, D. G., SCHULTZ, A. B., ALEXANDER, N. B. \& ASHTON-MILLER, J. A. 1996. Effects of age on rapid ankle torque development. J Gerontol A Biol Sci Med Sci, 51, M226-32. doi: 10.1093/gerona/51a.5.m226

TIEDEMANN, A., SHIMADA, H., SHERRINGTON, C., MURRAY, S. \& LORD, S. 2008. The comparative ability of eight functional mobility tests for predicting falls in communitydwelling older people. Age Ageing, 37, 430-5. doi: 10.1093/ageing/afn100

TIMSINA, L. R., WILLETTS, J. L., BRENNAN, M. J., MARUCCI-WELLMAN, H., LOMBARDI, D. A., COURTNEY, T. K. \& VERMA, S. K. 2017. Circumstances of fall-related injuries by age and gender among community-dwelling adults in the United States. PLoS One, 12, e0176561. doi: 10.1371/journal.pone.0176561 
TINETTI, M. E., SPEECHLEY, M. \& GINTER, S. F. 1988. Risk factors for falls among elderly persons living in the community. $N$ Engl $J$ Med, 319, 1701-7. doi: 10.1056/NEJM198812293192604

TORRES-OVIEDO, G. \& BASTIAN, A. J. 2010. Seeing is believing: effects of visual contextual cues on learning and transfer of locomotor adaptation. $J$ Neurosci, 30, 17015-22. doi: 10.1523/JNEUROSCI.4205-10.2010

TORRES-OVIEDO, G. \& BASTIAN, A. J. 2012. Natural error patterns enable transfer of motor learning to novel contexts. J Neurophysiol, 107, 346-56. doi: 10.1152/jn.00570.2011

TOWNSEND, M. A. 1985. Biped gait stabilization via foot placement. J Biomech, 18, 21-38.

TRICCO, A. C., THOMAS, S. M., VERONIKI, A. A., HAMID, J. S., COGO, E., STRIFLER, L., KHAN, P. A., ROBSON, R., SIBLEY, K. M., MACDONALD, H., RIVA, J. J., THAVORN, K., WILSON, C., HOLROYD-LEDUC, J., KERR, G. D., FELDMAN, F., MAJUMDAR, S. R., JAGLAL, S. B., HUI, W. \& STRAUS, S. E. 2017. Comparisons of Interventions for Preventing Falls in Older Adults: A Systematic Review and Meta-analysis. JAMA, 318, 1687-1699. doi: 10.1001/jama.2017.15006

TRICCO, A. C., THOMAS, S. M., VERONIKI, A. A., HAMID, J. S., COGO, E., STRIFLER, L., KHAN, P. A., SIBLEY, K. M., ROBSON, R., MACDONALD, H., RIVA, J. J., THAVORN, K., WILSON, C., HOLROYD-LEDUC, J., KERR, G. D., FELDMAN, F., MAJUMDAR, S. R., JAGLAL, S. B., HUI, W. \& STRAUS, S. E. 2019. Quality improvement strategies to prevent falls in older adults: a systematic review and network meta-analysis. Age Ageing, 48, 337 346. doi: 10.1093/ageing/afy219

TROMP, A. M., PLUIJM, S. M., SMIT, J. H., DEEG, D. J., BOUTER, L. M. \& LIPS, P. 2001. Fallrisk screening test: a prospective study on predictors for falls in community-dwelling elderly. J Clin Epidemiol, 54, 837-44.

TWEEDELL, A. J., RYAN, E. D., SCHARVILLE, M. J., ROSENBERG, J. G., SOBOLEWSKI, E. J. \& KLEINBERG, C. R. 2016. The influence of ultrasound measurement techniques on the age-related differences in Achilles tendon size. Exp Gerontol, 76, 68-71. doi: 10.1016/j.exger.2016.01.015

United Nations, Department of Economic and Social Affairs, Population Division. 2017. World Population Ageing 2017 (ST/ESA/SER.A/408).

VALKO, Y., ROSENGREN, S. M., JUNG, H. H., STRAUMANN, D., LANDAU, K. \& WEBER, K. P. 2016. Ocular vestibular evoked myogenic potentials as a test for myasthenia gravis. Neurology, 86, 660-8. doi: 10.1212/WNL.0000000000002383

VAN ASSELDONK, E. H., KOOPMAN, B. \& VAN DER KOOIJ, H. 2011. Locomotor adaptation and retention to gradual and sudden dynamic perturbations. IEEE Int Conf Rehabil Robot, 2011, 5975379. doi: 10.1109/ICORR.2011.5975379

VAN DE BERG, R. 2018. The Vestibular Implant: Feasibility in Humans. PhD Thesis, Maastricht University. doi: 10.26481/dis.20180920rb

VAN DE BERG, R., VAN TILBURG, M. \& KINGMA, H. 2015. Bilateral Vestibular Hypofunction: Challenges in Establishing the Diagnosis in Adults. ORL J Otorhinolaryngol Relat Spec, 77, 197-218. doi: 10.1159/000433549

VAN DER LINDEN, M. H., HENDRICKS, H. T., BLOEM, B. R. \& DUYSENS, J. 2009. Hitting a support surface at unexpected height during walking induces loading transients. Gait Posture, 29, 255-60. doi: 10.1016/j.gaitpost.2008.08.017 
VAN DER LINDEN, M. H., MARIGOLD, D. S., GABREELS, F. J. \& DUYSENS, J. 2007. Muscle reflexes and synergies triggered by an unexpected support surface height during walking. J Neurophysiol, 97, 3639-50. doi: 10.1152/jn.01272.2006

VAN DER ZIJDEN, A. M., GROEN, B. E., TANCK, E., NIENHUIS, B., VERDONSCHOT, N. \& WEERDESTEYN, V. 2012. Can martial arts techniques reduce fall severity? An in vivo study of femoral loading configurations in sideways falls. $J$ Biomech, 45, 1650-5. doi: 10.1016/j.jbiomech.2012.03.024

VAN DOOREN, T. S., LUCIEER, F. M. P., JANSSEN, A. M. L., KINGMA, H. \& VAN DE BERG, R. 2018. The Video Head Impulse Test and the Influence of Daily Use of Spectacles to Correct a Refractive Error. Front Neurol, 9, 125. doi: 10.3389/fneur.2018.00125

VAN HEDEL, H. J., BIEDERMANN, M., ERNI, T. \& DIETZ, V. 2002. Obstacle avoidance during human walking: transfer of motor skill from one leg to the other. J Physiol, 543, 709-17. doi: 10.1113/jphysiol.2002.018473

VAN HOOREN, B., MEIJER, K. \& MCCRUM, C. 2019. Attractive Gait Training: Applying Dynamical Systems Theory to the Improvement of Locomotor Performance Across the Lifespan. Front Physiol, 9, 1934. doi: 10.3389/fphys.2018.01934

VAN SCHOOTEN, K. S., PIJNAPPELS, M., RISPENS, S. M., ELDERS, P. J., LIPS, P., DAFFERTSHOFER, A., BEEK, P. J. \& VAN DIEËN, J. H. 2016. Daily-Life Gait Quality as Predictor of Falls in Older People: A 1-Year Prospective Cohort Study. PLoS One, 11, e0158623. doi: 10.1371/journal.pone.0158623

VAN SCHOOTEN, K. S., YANG, Y., FELDMAN, F., LEUNG, M., MCKAY, H., SIMS-GOULD, J. $\&$ ROBINOVITCH, S. N. 2018. The Association Between Fall Frequency, Injury Risk, and Characteristics of Falls in Older Residents of Long-Term Care: Do Recurrent Fallers Fall More Safely? J Gerontol A Biol Sci Med Sci, 73, 786-791. doi: 10.1093/gerona/glx196

VAN SWIGCHEM, R., GROEN, B. E., WEERDESTEYN, V. \& DUYSENS, J. 2009. The effects of time pressure and experience on the performance of fall techniques during a fall. $J$ Electromyogr Kinesiol, 19, 521-31. doi: 10.1016/j.jelekin.2007.12.004

VAN TILBURG, M. J., HERRMANN, B. S., GUINAN, J. J., JR. \& RAUCH, S. D. 2016. Increasing the Stimulation Rate Reduces cVEMP Testing Time by More Than Half With No Significant Difference in Threshold. Otol Neurotol, 37, 933-6. doi: 10.1097/MAO.0000000000001096

VANRENTERGHEM, J., GORMLEY, D., ROBINSON, M. \& LEES, A. 2010. Solutions for representing the whole-body centre of mass in side cutting manoeuvres based on data that is typically available for lower limb kinematics. Gait Posture, 31, 517-21. doi: 10.1016/j.gaitpost.2010.02.014

VASUDEVAN, E. V., GLASS, R. N. \& PACKEL, A. T. 2014. Effects of traumatic brain injury on locomotor adaptation. $J$ Neurol Phys Ther, 38, 172-82. doi: 10.1097/NPT.0000000000000049

VAUGHAN, C. L. \& O'MALLEY, M. J. 2005. Froude and the contribution of naval architecture to our understanding of bipedal locomotion. Gait Posture, 21, 350-62. doi: 10.1016/j.gaitpost.2004.01.011

VERHEYDEN, G. S., WEERDESTEYN, V., PICKERING, R. M., KUNKEL, D., LENNON, S., GEURTS, A. C. \& ASHBURN, A. 2013. Interventions for preventing falls in people after stroke. Cochrane Database Syst Rev, 5, CD008728. doi: 10.1002/14651858.CD008728.pub2 
VERMA, S. K., WILLETTS, J. L., CORNS, H. L., MARUCCI-WELLMAN, H. R., LOMBARDI, D. A. \& COURTNEY, T. K. 2016. Falls and Fall-Related Injuries among Community-Dwelling Adults in the United States. PLoS One, 11, e0150939. doi: 10.1371/journal.pone.0150939

VIEIRA, L. S., GOMES, A. P., BIERHALS, I. O., FARIAS-ANTUNEZ, S., RIBEIRO, C. G., MIRANDA, V. I. A., LUTZ, B. H., BARBOSA-SILVA, T. G., LIMA, N. P., BERTOLDI, A. D. \& TOMASI, E. 2018. Falls among older adults in the South of Brazil: prevalence and determinants. Rev Saude Publica, 52, 22. doi: 10.11606/s1518-8787.2018052000103

VILENSKY, J. A., COOK, J. A. \& COOPER, J. L. 1999. Stumbling corrective responses in healthy human subjects to rapid reversal of treadmill direction. J Electromyogr Kinesiol, 9, 161-71.

WALSH, G. S., LOW, D. C. \& ARKESTEIJN, M. 2018. Effect of stable and unstable load carriage on walking gait variability, dynamic stability and muscle activity of older adults. $J$ Biomech, 73, 18-23. doi: 10.1016/j.jbiomech.2018.03.018

WANG, X., MA, Y., WANG, J., HAN, P., DONG, R., KANG, L., ZHANG, W., SHEN, S., WANG, J., LI, D., ZHOU, M., WANG, L., NIU, K. \& GUO, Q. 2016. Mobility and Muscle Strength Together are More Strongly Correlated with Falls in Suburb-Dwelling Older Chinese. Sci Rep, 6, 25420. doi: 10.1038/srep25420

WANG, Y., BHATT, T., LIU, X., WANG, S., LEE, A., WANG, E. \& PAI, Y. C. 2019. Can treadmillslip perturbation training reduce immediate risk of over-ground-slip induced fall among community-dwelling older adults? $J$ Biomech, 84, 58-66. doi: 10.1016/j.jbiomech.2018.12.017

WELCH, T. D. \& TING, L. H. 2014. Mechanisms of motor adaptation in reactive balance control. PLoS One, 9, e96440. doi: 10.1371/journal.pone.0096440

WENGER, N. S., ROTH, C. P., SHEKELLE, P. G., YOUNG, R. T., SOLOMON, D. H., KAMBERG, C. J., CHANG, J. T., LOUIE, R., HIGASHI, T., MAClEAN, C. H., ADAMS, J., MIN, L. C., RANSOHOFF, K., HOFFING, M. \& REUBEN, D. B. 2009. A practice-based intervention to improve primary care for falls, urinary incontinence, and dementia. J Am Geriatr Soc, 57, 547-55. doi: 10.1111/j.1532-5415.2008.02128.x

WENGER, N. S., SOLOMON, D. H., ROTH, C. P., MACLEAN, C. H., SALIBA, D., KAMBERG, C. J., RUBENSTEIN, L. Z., YOUNG, R. T., SLOSS, E. M., LOUIE, R., ADAMS, J., CHANG, J. T., VENUS, P. J., SCHNELLE, J. F. \& SHEKELLE, P. G. 2003. The quality of medical care provided to vulnerable community-dwelling older patients. Ann Intern Med, 139, 7407.

WHIPPLE, R. H., WOLFSON, L. I. \& AMERMAN, P. M. 1987. The relationship of knee and ankle weakness to falls in nursing home residents: an isokinetic study. J Am Geriatr Soc, 35, 1320.

WHITNEY, S. \& MORRIS, L. O. 2006. Multisensory Impairment in Older Adults: Evaluation and Intervention. In: CALHOUN, K. H., EIBLING, D. E., WAX, M. K. \& KOST, K. M. (eds.) Geriatric Otolaryngology. New York, USA: Taylor \& Francis Group.

WINTER, D. A. 1995. Human balance and posture control during standing and walking. Gait \& Posture, 3, 193-214. doi: 10.1016/0966-6362(96)82849-9

WITTENBERG, E., THOMPSON, J., NAM, C. S. \& FRANZ, J. R. 2017. Neuroimaging of Human Balance Control: A Systematic Review. Front Hum Neurosci, 11, 170. doi: 10.3389/fnhum.2017.00170 
WOLFSON, L., JUDGE, J., WHIPPLE, R. \& KING, M. 1995. Strength is a major factor in balance, gait, and the occurrence of falls. J Gerontol A Biol Sci Med Sci, 50 Spec No, 64-7. doi: 10.1093/gerona/50a.special_issue.64

WOLPAW, J. R. 2010. What can the spinal cord teach us about learning and memory? Neuroscientist, 16, 532-49. doi: 10.1177/1073858410368314

WOLPAW, J. R. 2012. Harnessing neuroplasticity for clinical applications. Brain, 135, e215; author reply e216. doi: 10.1093/brain/aws017

WOLTRING, H. J. 1986. A Fortran package for generalized, cross-validatory spline smoothing and differentiation. Adv Eng Software, 8, 104-113.

WONG, A. K., LORD, S. R., STURNIEKS, D. L., DELBAERE, K., TROLLOR, J. N. \& CLOSE, J. C. 2013. Angiotensin system-blocking medications are associated with fewer falls over 12 months in community-dwelling older people. $J$ Am Geriatr Soc, 61, 776-81. doi: 10.1111/jgs. 12205

WUEHR, M., DECKER, J. \& SCHNIEPP, R. 2017. Noisy galvanic vestibular stimulation: an emerging treatment option for bilateral vestibulopathy. $J$ Neurol, 264, 81-86. doi: 10.1007/s00415-017-8481-4

WUEHR, M., NUSSER, E., DECKER, J., KRAFCZYK, S., STRAUBE, A., BRANDT, T., JAHN, K. \& SCHNIEPP, R. 2016. Noisy vestibular stimulation improves dynamic walking stability in bilateral vestibulopathy. Neurology, 86, 2196-202. doi: 10.1212/WNL.0000000000002748

WUEHR, M., SCHNIEPP, R., PRADHAN, C., ILMBERGER, J., STRUPP, M., BRANDT, T. \& JAHN, K. 2013. Differential effects of absent visual feedback control on gait variability during different locomotion speeds. Exp Brain Res, 224, 287-94. doi: 10.1007/s00221-0123310-6

XU, D. \& DREW, J. A. 2016. Cause, nature and care-seeking behaviour for injuries among community-dwelling older adults, USA, 2004-2013. Inj Prev, 22, 46-51. doi: 10.1136/injuryprev-2015-041583

YAMADA, M. \& ICHIHASHI, N. 2010. Predicting the probability of falls in community-dwelling elderly individuals using the trail-walking test. Environ Health Prev Med, 15, 386-91. doi: 10.1007/s12199-010-0154-1

YANG, F., BHATT, T. \& PAI, Y. C. 2013. Generalization of treadmill-slip training to prevent a fall following a sudden (novel) slip in over-ground walking. $J$ Biomech, 46, 63-9. doi: 10.1016/j.jbiomech.2012.10.002

YANG, F., CERECERES, P. \& QIAO, M. 2018a. Treadmill-based gait-slip training with reduced training volume could still prevent slip-related falls. Gait Posture, 66, 160-165. doi: 10.1016/j.gaitpost.2018.08.029

YANG, F. \& PAI, Y. C. 2014. Can sacral marker approximate center of mass during gait and slipfall recovery among community-dwelling older adults? $J$ Biomech, 47, 3807-12. doi: 10.1016/j.jbiomech.2014.10.027

YANG, F., WANG, T. Y. \& PAI, Y. C. 2014. Reduced intensity in gait-slip training can still improve stability. J Biomech, 47, 2330-8. doi: 10.1016/j.jbiomech.2014.04.021

YANG, J. F., STEPHENS, M. J. \& VISHRAM, R. 1998. Transient disturbances to one limb produce coordinated, bilateral responses during infant stepping. $J$ Neurophysiol, 79, 2329-37. doi: 10.1152/jn.1998.79.5.2329 
YANG, Y., MACKEY, D. C., LIU-AMBROSE, T., FELDMAN, F. \& ROBINOVITCH, S. N. 2016. Risk factors for hip impact during real-life falls captured on video in long-term care. Osteoporos Int, 27, 537-47. doi: 10.1007/s00198-015-3268-x

YANG, Y., VAN SCHOOTEN, K. S., SIMS-GOULD, J., MCKAY, H. A., FELDMAN, F. \& ROBINOVITCH, S. N. 2018b. Sex Differences in the Circumstances Leading to Falls: Evidence From Real-Life Falls Captured on Video in Long-Term Care. J Am Med Dir Assoc, 19, 130-135 e1. doi: 10.1016/j.jamda.2017.08.011

YASUMURA, S., HAGA, H., NAGAI, H., SUZUKI, T., AMANO, H. \& SHIBATA, H. 1994. Rate of falls and the correlates among elderly people living in an urban community in Japan. Age Ageing, 23, 323-7. doi: 10.1093/ageing/23.4.323

YIP, C. W., GLASER, M., FRENZEL, C., BAYER, O. \& STRUPP, M. 2016. Comparison of the Bedside Head-Impulse Test with the Video Head-Impulse Test in a Clinical Practice Setting: A Prospective Study of 500 Outpatients. Front Neurol, 7, 58. doi: 10.3389/fneur.2016.00058

ZALEWSKI, C. K. 2015. Aging of the Human Vestibular System. Semin Hear, 36, 175-96. doi: 10.1055/s-0035-1555120

ZENI, J. A., JR., RICHARDS, J. G. \& HIGGINSON, J. S. 2008. Two simple methods for determining gait events during treadmill and overground walking using kinematic data. Gait Posture, 27, 710-4. doi: 10.1016/j.gaitpost.2007.07.007

ZHONG, H., ROY, R. R., NAKADA, K. K., ZDUNOWSKI, S., KHALILI, N., DE LEON, R. D. \& EDGERTON, V. R. 2012. Accommodation of the spinal cat to a tripping perturbation. Front Physiol, 3, 112. doi: 10.3389/fphys.2012.00112 


\section{Acknowledgements}

"Everyone you will ever meet knows something you don't."

- Bill Nye

"An extremely Dutch question, like simultaneously expressing an appropriate amount of selfscepticism while pointing out the fact that other people are wrong, an attitude for which I am deeply in love."

- James Heathers, responding to a question from a Dutch listener

(Everything Hertz podcast episode 77: Promiscuous expertise) 
As stated in the title of this dissertation, the last few years really have been a trip to remember, in more ways than one. I am incredibly grateful to everyone who has directly or indirectly supported me during and prior to this period. Reflecting on how I got to this point, it is clear that it would not have been possible without the advice, education, support, collaboration, friendship and love of many, many people.

Kiros: Thank you for all of the opportunities that you have given me! From starting my master thesis with you in 2013 with no real prior experience in biomechanics to now at the completion of my $\mathrm{PhD}$, you always challenged and supported me in my development. You told me at the end of my masters when I asked about whether my knowledge was sufficient to begin a $\mathrm{PhD}$, that it was more a matter of will, because you have to learn so many new things during a $\mathrm{PhD}$ anyway. This has really stuck with me and with the support of my supervisors has pushed me to continue to work on my understanding of my topics. I am very proud of the team we have become over the last few years, together with Gaspar and Matthias, and look forward to continuing to work together!

Kenneth: I have and continue to really appreciate your supervision and support! You have given me a freedom that I think many students do not have, when it comes to exploring and developing their own identity as a researcher. I have never felt constrained in any way and so many positives have come out of this freedom. Nevertheless, you have always been there to provide advice and context and have helped me see the bigger picture or finer details of an issue when I needed it. Thank you also for your faith and confidence in me and my future; I hope I can prove them well placed! Thank you for everything!

Raymond: I have really appreciated our work together. Your enthusiasm and above all, your selfless dedication to your research and the eventual benefit of research to your patients is inspiring. I am very pleased that we will continue to work together on these topics. Thank you for your great collaboration and support!

Wiebren: Thank you for all of the support that you and the institute have given me. I have greatly enjoyed our interactions over the last few years and have learned a lot from you. Hopefully we will continue our work together soon!

Herman: You welcomed me and Kenneth into you and Raymond's projects with such enthusiasm and I am very grateful for this opportunity. Your support these last few years is greatly appreciated!

Dissertation Assessment Committee: Jeroen, Uwe, Ton, Mirjam and Hans, thank you all for dedicating your time and expertise to the evaluation of my dissertation and for your participation in the defence ceremony!

Paranymphs: Gaspar, thank you for your friendship and support these last years! Your advice and encouragement, especially in the early years of my project, really helped a lot! We have spent a lot of time working (and travelling) very hard (that have even led to you falling asleep mid-sentence) but despite this, you were always able to make things fun! Despite how busy or stressed you were, you always had time, energy and motivation to help others and I really admire this in you. And there is no one better to light up a wedding dancefloor! Matthias, auch bei dir möchte ich mich für die letzten Jahre ganz herzlich bedanken, denn ich schätze unsere Freundschaft wirklich sehr! Danke für den regen 
Austausch unserer gegenseitigen Arbeit, aber auch vor allem für die vielen wertschätzenden Unterhaltungen über alle möglichen Themen. Ich wünsche dir und Jenni alles alles Gute für eure gemeinsame Zukunft und deine letzte Phase deines $\mathrm{PhD}$.

A big thank you to all past and present members of the movement science group in Maastricht! Especially thanks to Kenneth, Hans, Maarten, Jos, Thamar, Marlies, Brenda, Guy for firstly, your education and later, your support and advice that have helped me on my way! Paul, special thanks to you for your time and support on the technical side of my projects - it would not have been possible without you! Pieter, thanks to you for your early advice and support as one of my first roommates during the $\mathrm{PhD}$ ! Bernard, I really enjoyed working next to you! We thought very similarly about many things and I gained a lot from our discussions! Your patients are very lucky to have you as their GP! Wai-Yan, my co-gait stability researcher in the group, it has been really great to learn from you and your work over the last few years, and it has been a lot of fun! Hans, my long-time desk neighbour, thanks for the years of camaraderie and the more and less office-appropriate discussions! I have learned everything from jokes to the details of EMG analysis from you and wouldn't have it any other way! And thanks for the great title idea! Li-Juan, the last few years have been great fun and I have enjoyed learning a lot about the applied or clinical side of (loco)motor learning from you. I'm looking forward to seeing the outcomes of your project soon! HQ, our time as roommates was too short, but great nonetheless! It has been great getting to know you and I can't wait to see the outcome of your studies after witnessing what feels like thousands of activpals going through the office! Wouter, thanks for all of your help with my measurements! The support during a very busy time is greatly appreciated! We miss you on the $2^{\text {nd }}$ floor! Kyra, Irene and Bas - the next batch of Kenneth's PhDs is shaping up nicely! Looking forward to continuing to see how you and the projects develop and to continuing to learn from you! Bart, it has been nice getting to know you and I am looking forward to working together more closely in the future!

Ein sehr großes Dankeschön an alle Mitarbeiter vom Institut für Bewegungs- und Sportgerontologie der Sporthochschule Köln für die Unterstützung und Zusammenarbeit der letzten Jahre! Besonderer Dank gilt Stefanie, dafür, dass ich mich immer willkommen fühlen durfte!

Harry, Desiree and Yolanda: Thank you all for the support these last few years! Whenever I have questions (simple, stupid or sensible) you are always quick and happy to help, and I really appreciate it!

Rik and Rachel: Thank you both for the support at the CAREN, especially at the beginning of my measurements!

Thanks to all of the Maastricht Vestibular Team for their accommodation and collaboration! Floor, thanks for all of the nice mornings at the CAREN and for the great collaboration! Also special thanks to you, Ellen and Sophie, for helping me learn and understand the vestibular tests! 
Also many thanks to the Vestibular Implant Team in Geneva for their collaboration! Especially to Angélica and Nils for their contribution to the first article in this dissertation!

Avril: I am very grateful for your support these last few years. Going from reading your papers in detail as a student, to working with you remotely on the review in my dissertation, to joining your lab for the summer last year has been a great experience and I have learned a lot from you. Thanks also to the entire SIMbL team, especially Andrew for your support during my visit!

Lotte: Thanks to you for the nice collaboration these last few years and for helping me complete my final paper. And thanks to Joris for helping us link our projects together!

Many thanks to all of the participants in the research within this dissertation!

The financial support of the Kootstra Talent Fellowship and the NUTRIM Graduate Programme are very much appreciated. My thanks to the committees for their faith and support! Thanks also to the various organisations that have awarded me travel grants: The Force and Motion Foundation, The BANCOM 2016 Organisers together with the NIH, The International Society of Biomechanics, and the De Luca Foundation. Many thanks also to the European Group for Research into Elderly and Physical Activity for their support with the 2016-2017 Young Researcher Award. Additionally, many thanks to the sponsors of the printing of this dissertation: Motek, Ultimateinstability, das Institut für Bewegungs- und Sportgerontologie (Deutsche Sporthochschule Köln), MED-EL and Prof. Dr. K. Eysel-Gosepath (HNO Cologne).

Finally, many thanks to Christina, Matthias and Kiros, and Hans, Eva, Irene, LiJuan and Kenneth for the help with my German and Dutch summaries!

Next, of course there are many people who I have had the pleasure of working with on topics and projects outside of my dissertation, who have nonetheless had a positive impact on my experience and development. To my Antwerp colleagues, especially Nolan, Anne, Evi and Vincent, thanks for the already very positive and productive collaborations together! Eva and Paul, thanks for involving me in your work and letting me learn more about the spine! Martijn, Jeannet and Anouk: Thank you for your efforts and collaboration at CIRO! Thijs: Our work together on and off the last few years has always been fun! Thanks for the nice ongoing work together! Lex and Sanne: Thank you both for the great teamwork on the systematic reviews course and especially for helping me with my first larger teaching role! Hannah, Stephanie, Sabine and Frieder: Thanks for sharing in the NMS Twitter journey - it has been nice getting to know you and helping to spread the department's work! Special thanks to the ISPGR Communications Committee members for the nice work together these last two years, especially to Kim who invited me to get involved! Thanks to the staff at Motek, especially Frans and Sanne, for your support and engagement over the last few years! Thanks to all of the MED-EL staff for their interest in and support of my work! And thanks to Paul and Ultimateinstability for your interest and support! Thanks to Kathrin for the early 
collaborations on our unilateral vestibulopathy papers and the support! Finally, thanks to Alice and team in Leuven for the open discussions and collaboration so far!

Many thanks to all of my past and current interns for their enthusiasm and help these last few years: Andrea, Juan, Shanice, Ray, Nathalie, Cas, Joost, Bas, Ward, Clemence, Yiannis, Simone, Isabelle, Twan. Special thanks especially to Marissa, for your extended effort and assistance at the beginning of my study! To Alexander, thank you for helping me deepen my understanding of our measurement methods! Pamela and Daniella, your extra efforts for the review papers are greatly appreciated! Julia, many thanks for your help with the last stages of my study over the last year!

My thanks also to Jogchum and all of the staff and PIs of the Department of Nutrition and Movement Science, as well as Annemie and NUTRIM, for their support and faith in my potential!

Many of the opportunities and experiences that led me to this point came from my experiences in sports and similarly some of the most valuable lessons and experiences that have helped me get to this point have come from sports. For these, I would like to thank all of my past teachers and coaches at the Dalziel PE Dept., Lau Gar Kung Fu, Carluke Predators, Motherwell AC, Clyde Valley Falcons and Hawks, Augustana Vikings, Stirling Clansmen and Sydney Uni AC, as well as Davie Easton and Linda Duddy. Likewise, many thanks to all of my club- and teammates over the years! Many thanks to all of my previous athletes at Motherwell AC for the pleasure of coaching them. Ebenso, vielen vielen Dank an alle meine aktuellen und ehemaligen Athleten und Athletinnen des ASV Köln! Conor, thanks for the support and collaboration!

Finally, I would like to thank all of our friends for their kindness, love and support!

Esther und Etienne, ihr habt so viel für uns gemacht und es ist wirklich nicht übertrieben, wenn ich sage, dass diese Arbeit ohne eure Unterstützung viel mehr Zeit und Nerven gekostet hätte. Vielen Dank für Alles!

Andy and Bruna, thank you for your friendship and support! You have been there for me and us for a very long time, no matter whether you were nearby or on the other side of the world! Andy, many things have changed for us, but our friendship stands the test of time, distance and country. I am thankful for you always being there for me!

Hermann und Sigrid, ihr beide habt mich und uns seit mehr als zehn Jahren auf die unterschiedlichste Art und Weise unterstützt, mich auf diesem Weg begleitet und ich kann euch gar nicht genug dafür danken! Vor allem in den letzten zwei Jahren wart ihr immer für uns da, wenn wir wegen unserer Arbeit Hilfe mit Alex benötigt haben. Der „SommerWille", die Zielstrebigkeit etwas zu erreichen und eure Hingabe für die Familie war mir immer ein Vorbild und wird mich weiterhin auf meinem Weg begleiten. Matthias, auch bei dir möchte ich mich ganz besonders bedanken, dass du so unermüdlich zu uns nach Köln kommst und dich ganz besonders toll um Alex kümmerst. Das weiß ich sehr zu schätzen. Außerdem möchte ich mich bei allen Familienmitgliedern der SommerFamilie für eure wertschätzende Ermunterung und euer reges Interesse bedanken.

To all of my family in Scotland, thank you for the years of encouragement and support! Growing up in such a supporting family has been a blessing! 
To Granny, the relationship between children and their grandparents is a very special one and I also see this now with Alex. It is also difficult to put into words, because Grandparents play so many important roles in their Grandchildren's lives. Thank you for all of your love and encouragement! I am extremely lucky, proud and grateful to be your grandson - thank you for being my Granny and everything that that entails!

David, we have spent many of the last ten years apart, but nevertheless when we see each other, it seems like many things don't change. We are still the same brothers that played, argued, competed and laughed together and it always feels like home when we are together, whether we are actually in Motherwell or not. Thank you for always being there for me!

To my parents, thank you for everything that you have done, and do, for me, and for us! You have supported me in all of my ambitions and provided me with so many opportunities that have led to where I am today. So many of the steps I have taken in my life have been so much easier than they might have been due to your encouragement and support, and due to me having the knowledge and comfort that you were there for me, whether I would be successful or not. You have also taught me so much about the important things in life, including by your example and your dedication to helping others in so many different ways. The example you have set for me as parents and as people is second to none and I am very thankful for that. Finally, thank you for all of your help in these last few years. Despite the distance, you have been there for us and Alex whenever we needed you during this new and busy time in our lives.

Alex, it will be a while until you read this, but even after only two years, there are many things to thank you for: the joy you bring to the people around you; the cheeky smiles; the extreme bursts of intensity, passion and excitement for the small everyday-things in life like aeroplanes, dogs and splashing in puddles; the ability to make me forget everything else I was thinking about with a smile or a giggle; and the new perspective you have given me on the important things in life.

Christina, thank you for everything that you are and do! My love, my best friend, my wife, my person. You have helped me become a better version of myself in every way. There is no one who inspires, motivates, challenges or grounds me like you do. You have given me the courage to push my boundaries on so many fronts and I have achieved so much due to your love and support. I am so full of pride and respect for you and how you approach life, work, and even small day-to-day tasks (it is worth noting that people have won Olympic medals with less focus and determination than you have shown while helping people move house!). All this to say that you being who you are, inspires me to be the best I can be. I love you! Thank you for everything! 
Curriculum Vitae 


\section{Publication List}

\section{Submitted}

McCrum C, Lucieer F, van de Berg R, Willems P, Pérez Fornos A, Guinand N, Karamanidis K, Kingma H, Meijer K. Is faster always better? The walking speed-dependency of gait variability in bilateral vestibulopathy. Preprint at bioRxiv. doi: $\underline{10.1101 / 413955}$

Herssens N, Verbecque E, McCrum C, Meijer K, Van De Berg R, Saeys W, Vereeck L, Van Rompaey V, Hallemans A. A systematic review on balance performance in patients with bilateral vestibulopathy.

\section{Accepted}

McCrum C, Karamanidis K, Grevendonk L, Zijlstra W, Meijer K. Older adults demonstrate interlimb transfer of reactive gait adaptations to repeated unpredictable gait perturbations. GeroScience. doi: 10.1007/s11357-019-00130-x Preprint at bioRxiv. doi: 10.1101/673574

\section{Published}

Karamanidis K, Epro G, McCrum C, König M. (2019) Improving trip and slip-resisting skills in older people: perturbation dose matters. Exercise and Sport Sciences Reviews. doi: 10.1249/JES.0000000000000210

Jeras NMJ, Bovend'Eerdt TJH, McCrum C. (2019) Biomechanical mechanisms of jumping performance in youth elite female soccer players. Journal of Sports Sciences, doi: $\underline{10.1080 / 02640414.2019 .1674526}$

Herssens N, McCrum C. (2019) Stimulating balance: recent advances in vestibular stimulation for balance and gait. Journal of Neurophysiology, 122(2): 447-450. doi: 10.1152/jn.00851.2018

Jacobs E, Senden R, McCrum C, van Rhijn LW, Meijer K, Willems PC. (2019) The effect of a semirigid thoracolumbar orthosis on gait and sagittal alignment in patients with an osteoporotic vertebral compression fracture. Clinical Interventions in Aging, 14: 671-680. doi: 10.2147/CIA.s199853

Jacobs E, McCrum C, Senden R, van Rhijn LW, Meijer K, Willems PC. (2019) Gait in patients with symptomatic osteoporotic vertebral compression fractures over six months recovery. Aging Clinical and Experimental Research, doi: 10.1007/s40520-019-01203-9

McCrum C, Willems P, Karamanidis K, Meijer K. (2019) Stability-normalised walking speed: a new approach for human gait perturbation research. Journal of Biomechanics, doi: 10.1016/j.jbiomech.2019.02.016 Preprint at_bioRxiv. doi: $\underline{10.1101 / 314757}$

Jafarnezhadgero AA, Madadi M, McCrum C, Karamanidis K. (2019) Effects of corrective training on drop landing ground reaction force characteristics and lower limb kinematics in older adults with genu valgus: A randomized controlled trial. Journal of Aging and Physical Activity, 27(1): 9-17. doi: 10.1123/japa.2017-0315

Van Hooren B, Meijer K, McCrum C. (2019) Attractive gait training: applying dynamical systems theory to the improvement of locomotor performance across the lifespan. Frontiers in Physiology, 9: 1934. doi: 10.3389/fphys.2018.01934 
McCrum C, Karamanidis K, Willems P, Zijlstra W, Meijer K. (2018) Retention, savings and interlimb transfer of reactive gait adaptations in humans following unexpected perturbations. Communications Biology, 1: 230. doi: 10.1038/s42003-018-0238-9

König M, Hemmers S, Epro G, McCrum C, Ackermans TM, Hartmann U, Karamanidis K. (2018) Matching participants for triceps surae muscle strength and tendon stiffness does not eliminate age-related differences in mechanical power output during jumping. Frontiers in Physiology, 9: 1345. doi: 10.3389/fphys.2018.01345

Holzer D, Epro G, McCrum C, Doerner J, Luetkens JA, Scheef L, Kukuk GM, Boecker H, Mierau A, Brüggeman G-P, Maganaris CN, Karamanidis K. (2018) The role of muscle strength on tendon adaptability in old age. European Journal of Applied Physiology, 118(11): 22692279. doi: $10.1007 / \mathrm{s} 00421-018-3947-3$

McCrum C. (2018) Falls among older people-are intervention duration and specificity the keys to making a difference? The BMJ, 361: k2213. doi: 10.1136/bmj.k2213

Epro G, Mierau A, McCrum C, Leyendecker M, Brüggemann G-P, Karamanidis K. (2018) Retention of gait stability improvements over 1.5 years in older adults: effects of perturbation exposure and triceps surae neuromuscular exercise. Journal of Neurophysiology, 119(6): 2229-2240. doi: 10.1152/jn.00513.2017

Epro G, McCrum C, Mierau A, Leyendecker M, Brüggemann G-P, Karamanidis K. (2018) Effects of triceps surae muscle strength and tendon stiffness on the reactive dynamic stability and adaptability of older female adults during perturbed walking. Journal of Applied Physiology, 124(6): 1541-1549. doi: 10.1152/japplphysiol.00545.2017

McCrum C, Leow P, Epro G, König M, Meijer K, Karamanidis K. (2018) Alterations in leg extensor muscle-tendon unit biomechanical properties with ageing and mechanical loading. Frontiers in Physiology, 9: 150. doi: 10.3389/fphys.2018.00150

McCrum C, Oberländer KD, Epro G, Krauss P, James DC, Reeves ND, Karamanidis K. (2018) Loading rate and contraction duration effects on in vivo human Achilles tendon mechanical properties. Clinical Physiology and Functional Imaging, 38:517-523. doi: 10.1111/cpf.12472

Gerards MHG*, McCrum C*, Mansfield A, Meijer K. (2017) Perturbation-based balance training for falls reduction among older adults: current evidence and implications for clinical practice. Geriatrics \& Gerontology International, 17: 2294-2303. doi: 10.1111/ggi.13082 *Joint first authors.

McCrum C, Gerards MHG, Karamanidis K, Zijlstra W, Meijer K. (2017) A systematic review of gait perturbation paradigms for improving reactive stepping responses and falls risk among healthy older adults. European Review of Aging and Physical Activity, 14: 3. doi: 10.1186/s11556-017-0173-7

McCrum C, Eysel-Gosepath K, Epro G, Meijer K, Savelberg HHCM, Brüggemann GP, Karamanidis K. (2017) Associations between bipedal stance stability and locomotor stability following a trip in unilateral vestibulopathy. Journal of Applied Biomechanics, 33(2): 112-117. doi: 10.1123/jab.2016-0004

Ackermans TMA, Epro G, McCrum C, Oberländer KD, Suhr F, Drost MR, Meijer K, Karamanidis K. (2016) Aging and the effects of a half marathon on Achilles tendon length-tension properties. European Journal of Applied Physiology, 116: 2281-2292. doi: 10.1007/s00421016-3482-z

Eysel-Gosepath K, McCrum C, Epro G, Brüggemann GP, Karamanidis K. (2016) Visual and proprioceptive contributions to postural control of upright stance in unilateral 
vestibulopathy. Somatosensory \& Motor Research, 33(2):72-78. doi: $\underline{10.1080 / 08990220.2016 .1178635}$

McCrum C, Essers JMN, Jie LJ, Liu WY, Meijer K. (2016) Commentary: Older adults can improve compensatory stepping with repeated postural perturbations. Frontiers in Aging Neuroscience, 8: 111. doi: 10.3389/fnagi.2016.00111

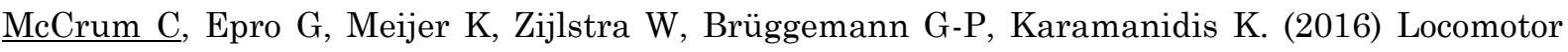
stability and adaptation during perturbed walking across the adult female lifespan. Journal of Biomechanics, 49(7): 1244-1247. doi: 10.1016/j.jbiomech.2016.02.051

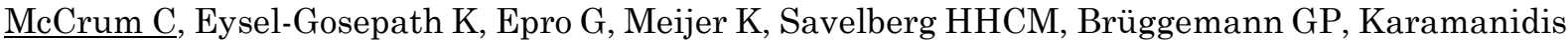
K. (2014) Deficient recovery response and adaptive feedback potential in dynamic gait stability in unilateral peripheral vestibular disorder patients. Physiological Reports, 2(12): e12222. doi: 10.14814/phy2.12222

\section{Consortium Contributions}

Brown P, RELISH Consortium, Zhou Y. (2019) Large expert-curated database for benchmarking document similarity detection in biomedical literature search. Database. doi: $\underline{10.1093 / \text { database/baz085 }}$

\section{Acknowledged Contributions}

Epro G, Mierau A, Doerner J, Luetkens JA, Scheef L, Kukuk GM, Boecker H, Maganaris CN, Brüggemann G-P, Karamanidis K. (2017) The Achilles tendon is mechanosensitive in older adults: adaptations following 14 weeks versus 1.5 years of cyclic strain exercise. Journal of Experimental Biology 220: 1008-1018. doi: 10.1242/jeb.146407

Karamanidis K, Travlou A, Krauss P, Jaekel U. (2016) Use of a Lucas-Kanade-Based Template Tracking Algorithm to Examine In Vivo Tendon Excursion during Voluntary Contraction Using Ultrasonography. Ultrasound in Medicine \& Biology 42(7): 1689-700. doi: 10.1016/j.ultrasmedbio.2016.02.019

\section{National Journals}

Karamanidis K, Epro G, König M, McCrum C, Ackermans T, Thomaskamp H-J, Schade F. Strukturanalyse der Muskel-Sehnen-Einheit von Spitzensportlern im Jahresverlauf. (2016) Leistungssport. 4.

\section{Conference Proceedings}

McCrum C, Karamanidis K, Grevendonk L, Willems P, Zijlstra W, Meijer K. (2019) Interlimb transfer of reactive gait adaptations in older but not young adults during unpredictable perturbations. (Oral presentation at the Dutch Society for Movement Science (VvBN) PhD Day, Groningen, The Netherlands, November 2019).

Herssens N, Verbecque E, Saeys W, Vereeck L, Van Rompaey V, McCrum C, Meijer K, Hallemans A. (2019) P 008 - Balance performance in bilateral vestibulopathy in relation to sensorimotor integration. (Poster presentation at the European Society of Movement Analysis in Adults and Children (ESMAC) Annual Meeting, Amsterdam, The Netherlands, September 2019). Published in Gait and Posture, doi: 10.1016/j.gaitpost.2019.07.146 
McCrum C. (2019) Targets for task-specific exercise-based fall risk assessment and intervention. (Poster presentation at the 20th International Conference on Falls and Postural Stability, London, UK, September 2019).

McCrum C, Karamanidis K, Willems P, Zijlstra W, Meijer K. (2019) Retention, savings and interlimb transfer of reactive gait adaptations in humans following unexpected perturbations. (Poster presentation at the International Society of Posture and Gait Research World Congress, Edinburgh, Scotland, July 2019).

Herssens N, Verbecque E, Saeys W, Vereeck L, Van Rompaey V, McCrum C, Meijer K, Hallemans A. (2019) Balance performance in bilateral vestibulopathy in relation to sensorimotor integration (Poster presentation at the International Society of Posture and Gait Research World Congress, Edinburgh, Scotland, July 2019).

McCrum C, Huntley A, Shinkel-Ivy A, Mansfield A. (2019) Lateral stability during anterior and posterior support surface perturbations in people with chronic stroke. (Poster presentation at the International Society of Posture and Gait Research World Congress, Edinburgh, Scotland, July 2019).

McCrum C. (2019) Advanced rehabilitation technology for innovative studies on gait stability and fall-risk. (Invited workshop presentation at the International Society of Posture and Gait Research World Congress, Edinburgh, Scotland, July 2019).

Gerards MHG, McCrum C, Mansfield A, Meijer K. (2019) Perturbation-based balance training for falls reduction among older adults: current evidence and implications for clinical practice. (Poster presentation at the World Confederation for Physical Therapy (WCPT) Congress, Geneva, Switzerland, May 2019).

McCrum C, Karamanidis K, Willems P, Zijlstra W, Meijer K. (2018) Retention, savings and interlimb transfer of reactive gait adaptations following unexpected perturbations. (Poster presentation at the Annual NUTRIM Symposium, Maastricht, The Netherlands, December 2018).

McCrum C, Willems P, Karamanidis K, Meijer K (2018). Stability-normalised walking speed: a new approach for human gait perturbation research. (Oral presentation at the World Congress of Biomechanics, Dublin, Ireland, July 2018).

McCrum C, Willems P, Lucieer F, van de Berg R, Kingma H, Karamanidis K, Meijer K (2018). The effects of walking speed and bilateral vestibulopathy on spatiotemporal gait parameters and their variability: preliminary results. (Poster presentation at the World Congress of Biomechanics, Dublin, Ireland, July 2018).

McCrum C, Willems P, Karamanidis K, Meijer K (2018). Adaptation and inter-limb transfer of gait stability following perturbations in young and older adults assessed using stabilitynormalised walking speeds. (Oral presentation at the 23rd annual Congress of the European College of Sport Science (ECSS), Dublin, Ireland, July 2018).

McCrum C, Willems P, Lucieer F, van de Berg R, Kingma H, Karamanidis K, Meijer K (2018). Spatiotemporal parameters and variability with changes in gait velocity in bilateral vestibulopathy. (Poster presentation at the XXX Bárány Society Meeting, Uppsala, Sweden, June 2018). Published in Journal of Vestibular Research, 28(1-2):193. doi: 10.3233/VES$\underline{180635}$

König M, Epro G, McCrum C, Bädorf M, Schade F \& Karamanidis K. Monitoring muscle-tendon adaptation in elite athletes: Preliminary data from a 1-year longitudinal investigation. 
(Oral presentation at the 22nd annual Congress of the European College of Sport Science (ECSS), Essen, Germany, July 2017).

McCrum C, Oberländer KD, Epro G, Krauss P, Reeves ND, Karamanidis K. Loading rate and contraction type effects on the human Achilles tendon force-elongation relationship. (Oral presentation at the 22nd annual Congress of the European College of Sport Science (ECSS), Essen, Germany, July 2017).

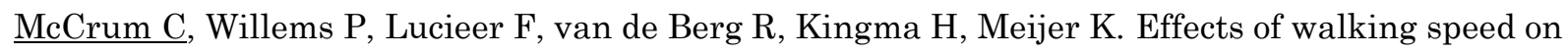
spatiotemporal gait characteristics and their variability in patients with bilateral vestibulopathy: preliminary observations (Oral presentation at the International Society of Posture and Gait Research (ISPGR) World Congress, Fort Lauderdale, USA, June 2017).

Epro G, König M, McCrum C, Bädorf M, Schade F \& Karamanidis K. Muscle-tendon adaptation monitoring in elite athletes: preliminary results from a longitudinal investigation (Oral presentation at the 35th International Conference on Biomechanics in Sports, Cologne, Germany, June 2017).

König M, Hemmers S, McCrum C, Epro G, Ackermans T, Hartmann U \& Karamanidis K. Matching triceps surae muscle strength and tendon stiffness eliminates age-related differences in drop-jump performance. (Poster presentation at the 35th International Conference on Biomechanics in Sports, Cologne, Germany, June 2017).

McCrum C, Willems P, Lucieer F, van de Berg R, Kingma H, Meijer K. Characterising gait over different walking speeds in patients with bilateral vestibular loss: preliminary results (Poster presentation at the $2^{\text {nd }}$ International Congress on NeuroRehabilitation and Neural Repair, Maastricht, The Netherlands, May 2017).

Epro G, McCrum C, Mierau A, Leyendecker M, Brüggemann G-P \& Karamanidis K. Effect of triceps surae muscle-tendon unit mechanical properties on gait stability and adaptability in older female adults. (Poster presentation at the 2017 BASES Biomechanics Interest Group (BIG), Portsmouth, United Kingdom, April 2017. Human Kinetics Best Student Poster Presentation Award 1st place).

König M, Epro G, McCrum C, Bädorf M, Schade F \& Karamanidis K. The effect of habitual athletics training on muscle and tendon adaptation in young and older elite athletes. (Oral presentation at the BASES Biomechanics Interest Group (BIG) Meeting, Portsmouth, United Kingdom, April 2017. Best Oral Presentation Award 2nd place).

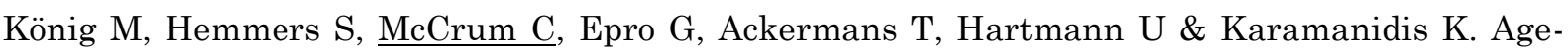
related differences in drop-jump performance are eliminated by matching triceps surae muscle strength and Achilles tendon stiffness. (Poster presentation at the BASES Biomechanics Interest Group (BIG) Meeting, Portsmouth, UK, April 2017).

König M, Epro G, McCrum C, Ackermans T, Schade F \& Karamanidis K. Muscle and tendon adaptation in young and older adult athletes: A combined longitudinal and cross sectional investigation. (Oral presentation at the 10. Jahrestagung der Deutschen Gesellschaft für Biomechanik, Hannover, Germany, March 2017).

McCrum C, Oberländer KD, Epro G, Krauss P, Reeves ND, Karamanidis K. Effects of loading rate and contraction type on the in vivo measurement of Achilles tendon mechanical properties. (Poster presentation at the Annual NUTRIM Symposium, Maastricht, The Netherlands, December 2016). 
Gerards M, McCrum C, Mansfield A, Meijer K. Perturbation-based balance training for falls reduction in the elderly: current evidence and clinical implications. (Poster presentation at the Annual NUTRIM Symposium, Maastricht, The Netherlands, December 2016).

Epro G, Mierau A, Leyendecker M, McCrum C, Brüggemann G-P, Karamanidis K. Effects of triceps surae muscle strength on gait stability and adaptability in older adults. (Poster presentation at the Dutch Society for Movement Science (VvBN) PhD Day, Maastricht, The Netherlands, November 2016).

König M, Hemmers S, McCrum C, Epro G, Ackermans T, Hartmann U, Karamanidis K. Age-related differences in drop jump performance are eliminated when triceps surae mechanical properties are matched. (Poster presentation at the Dutch Society for Movement Science (VvBN) PhD Day, Maastricht, The Netherlands, November 2016).

McCrum C, Epro G, Meijer K, Zijlstra W, Brüggemann G-P, Karamanidis K. Gait Adaptability and Stability during Perturbed Walking in Young, Middle-Aged and Older Adults. (Poster presentation at the Dutch Society for Movement Science (VvBN) PhD Day, Maastricht, The Netherlands, November 2016).

McCrum C, Willems P, van de Berg R, Cavuscens S, Guinand N, Guyot JP, Perez-Fornos A, Marcellis R, Ranieri M, Senden R, Stokroos R, Zijlstra W, Karamanidis K, Meijer K, Kingma H. Preliminary observations of the acute effects of vestibular nerve stimulation on stride length and time in two patients with bilateral vestibular hypofunction. (Oral presentation at the European Society of Movement Analysis in Adults and Children (ESMAC) 25th Annual Meeting, Seville, Spain, September 2016). Published in Gait and Posture, 49(S2):124. doi: 10.1016/j.gaitpost.2016.07.179

McCrum C, Epro G, Eysel-Gosepath K, Savelberg HCM, Brüggemann G-P, Meijer K, Zijlstra W, Karamanidis K. Locomotor Stability Control and Vestibular Function among Older Adults: Implications for Falls Prevention and Research. (Oral presentation at the 11th joint Conference on Motor Control \& Learning, Biomechanics \& Training of the German Society of Sports Sciences, Darmstadt, Germany, September 2016).

König M, Hemmers S, McCrum C, Epro G, Ackermans T, Hartmann U, Karamanidis K. Matching participants for triceps surae mechanical properties eliminates age-related differences in drop jump performance. (Poster presentation at the 11th joint Conference on Motor Control \& Learning, Biomechanics \& Training of the German Society of Sports Sciences, Darmstadt, Germany, September 2016).

Epro G, Mierau A, Leyendecker M, McCrum C, Brüggemann G-P, Karamanidis K. Effects of triceps surae contractile strength on gait stability and adaptability in older adults. (Poster presentation at the 11th joint Conference on Motor Control \& Learning, Biomechanics \& Training of the German Society of Sports Sciences, Darmstadt, Germany, September 2016).

McCrum C, Gerards M, Karamanidis K, Zijlstra W, Meijer K. A systematic review of gait perturbation paradigms for improving reactive stepping responses during walking and reducing falls in older people. (Mini-Oral presentation at the 21st annual Congress of the European College of Sport Science, Vienna, Austria, July 2016).

McCrum C, Epro G, Meijer K, Zijlstra W, Brüggemann G-P, Karamanidis K. Gait Adaptability and Stability during Perturbed Walking in Young, Middle-Aged and Older Adults. (Poster presentation at Biomechanics and Neural Control of Movement (BANCOM) 2016 conference, Mt. Sterling, OH, USA, June 2016). 
McCrum C, Willems P, Senden R, Karamanidis K, Kingma H, Zijlstra W, Meijer K. Assessing locomotor plasticity and stability with the Computer Assisted Rehabilitation Environment. (Poster presentation at the Annual NUTRIM Symposium, Maastricht, The Netherlands, December 2015).

McCrum C, Eysel-Gosepath K, Epro G, Meijer K, Savelberg HHCM, Brüggemann GP, Karamanidis K. Stability of Locomotion and Bipedal Stance in Unilateral Vestibulopathy. (Poster presentation at the Society for Movement Analysis Laboratories in the Low Lands (SMALLL) Annual Congress, Maastricht, The Netherlands, Novemeber 2015).

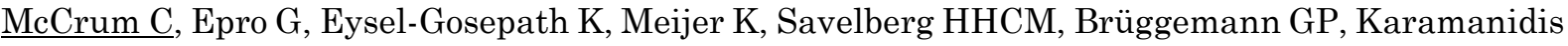
K. Stability recovery after tripping is not predicted by static posturography in vestibular disorder patients. (Poster presentation at the Dutch Society for Movement Science (VvBN) PhD Day, Enschede, The Netherlands, September 2015).

McCrum C, Eysel-Gosepath K, Epro G, Brüggemann GP, Karamanidis K. Sensory contributions to standing balance in unilateral vestibulopathy. (Poster presentation at the 7th International Posture Symposium, Smolenice Castle, Slovakia, September 2015).

McCrum C, Willems P, Karamanidis K, Kingma H, Zijlstra W, Meijer K. A protocol to assess locomotor plasticity and dynamic gait stability using the Computer Assisted Rehabilitation Environment. (Oral presentation at the 7th International Posture Symposium, Smolenice Castle, Slovakia, September 2015).

McCrum C, Eysel-Gosepath K, Epro G, Meijer K, Savelberg HHCM, Brüggemann GP, Karamanidis K. Static postural control does not strongly predict dynamic gait stability recovery following a trip in adults with and without vestibular dysfunction. (Oral presentation at the 20th annual Congress of the European College of Sport Science, Malmö, Sweden, June 2015).

McCrum C, Eysel-Gosepath K, Epro G, Meijer K, Savelberg HHCM, Brüggemann GP, Karamanidis K. Dynamic gait stability following tripping and static postural control in vestibular patients and healthy adults. (Oral presentation at the 9. Jahrestagung der Deutschen Gesellschaft für Biomechanik, Bonn, Germany, May 2015). 


\section{About the Author}

Christopher McCrum was born on the $4^{\text {th }}$ of August 1990 in Glasgow, Scotland. He attended Dalziel High School in Motherwell, Scotland, after which he received an International Merit scholarship to begin his university education at Augustana College (Rock Island, Illinois, USA) where he studied psychology and health education and was a member of the NCAA Division III Augustana Vikings American Football team from 2008 to 2009. From September 2009, Christopher continued at University of Stirling, Scotland, with a semester in 2011 at The University of Sydney, Australia. He graduated in 2013 with a Bachelor

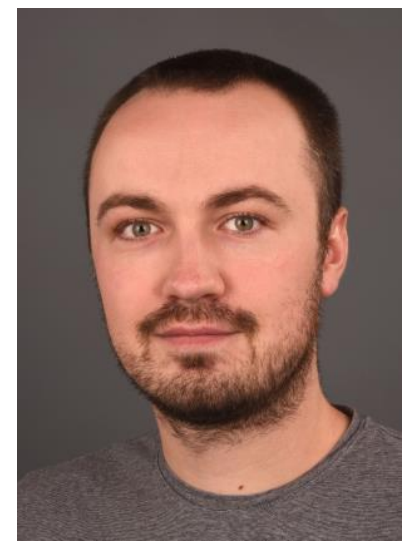
with Honours in Psychology and Sports Studies. Christopher then enrolled in the Master Biology of Human Performance and Health at Maastricht University, from which he graduated in August 2014. From December 2013 until July 2014, Christopher completed his master internship and thesis at the Institute of Biomechanics and Orthopaedics, German Sport University Cologne, Germany with Dr. Kiros Karamanidis, where he studied how walking stability following repeated tripping is affected in people with vestibulopathy and assisted in the PhD project of Dr. Gaspar Epro on triceps surae muscle-tendon unit mechanosensitivity and gait stability in older adults. Christopher's work during this period was awarded with the runner up prize of the SMALLL (Society for Movement Analysis Laboratories in the Low Lands) Student Award.

In December 2014, Christopher received the Kootstra Talent Fellowship from Maastricht University Medical Centre followed by a NUTRIM Graduate Programme grant in June 2015. Both grants allowed him to complete his doctoral research between 2015 and 2019 within the Department of Nutrition and Movement Sciences (Maastricht University), in collaboration with the Institute of Sport and Movement Gerontology, German Sport University Cologne, Germany under the guidance of Dr. Kenneth Meijer and Dr. Kiros Karamanidis, with support from Dr. Raymond van de Berg, as well as Prof. Wiebren Zijlstra and Prof. Herman Kingma. During this time, he focused on how walking stability is affected by age and vestibulopathy and how it can be assessed and improved using mechanical disturbances to balance in a safe, controlled environment, as presented in this dissertation.

Christopher has presented his work at various international conferences with the support of multiple travel awards from the Force and Motion Foundation, the NIH and the De Luca Foundation. In 2017, Christopher received the 2016-2017 Young Researcher Award of EGREPA (European Group for Research into Elderly and Physical Activity). Since 2017, he serves as a member of the Communications Committee of the International Society of Posture and Gait Research (ISPGR). In 2018, he received an International Travel Grant from the International Society of Biomechanics (ISB) and spent July to August 2018 visiting Dr. Avril Mansfield's Safe Independent Mobility Lab (SIMbL) at the Toronto Rehabilitation Institute, Canada.

Since September 2019, Christopher continues his research on the assessment and improvement of human balance and walking as an Assistant Professor in the Department of Nutrition and Movement Sciences (Maastricht University). 
